\title{
Behavioural Pharmacology Of Novel Kappa Opioid Compounds
}

By

\section{Aashish Sultan Morani}

\author{
A thesis to be submitted to Victoria University of Wellington \\ in fulfilment of the requirements for the degree of \\ Doctor of Philosophy
}

\section{Victoria University of Wellington}




\section{CONTENTS}

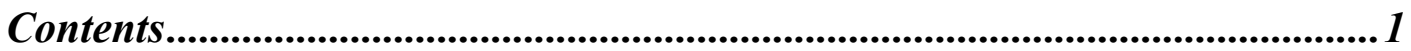

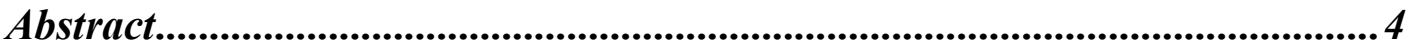

Acknowledgements...................................................................................................6

List of figures ........................................................................................................... 8

List of tables ...........................................................................................................9

List of abbreviations....................................................................................10

Chapter 1.General Introduction.................................................................................11

1.1. Current pharmacotherapies for addiction......................................................... 15

1.2. The addiction cycle ................................................................................................ 18

1.3. Animal models of addiction.................................................................................. 20

1.3.1. Operant conditioning in studying addictive behaviours .......................... 20

1.3.2. Non-operant conditioning in studying aversive behaviours................... 21

1.4. Cocaine addiction in pre-clinical models ........................................................... 23

1.4.1. Effect of cocaine on dopamine transporter function ................................. 23

1.5. The kappa opioid receptors .................................................................................. 27

1.5.1. Negative feedback loop and dynorphin/kappa opioid system .................. 29

1.5.2. Kappa opioid receptor agonists.................................................................. 31

1.5.3. Kappa opioid agonists in pre-clinical models of addiction ......................... 33

1.5.3.1. Arylacetamides.................................................................................... 33

1.5.3.2. Benzomorphans .................................................................................. 34

1.5.3.3. Epoxymorphinans.............................................................................. 34

1.5.3.4. Phytochemical KOPr agonists ......................................................... 34

1.5.4. Mechanism of KOPr agonist induced anti-addiction effects..................... 36

1.5.5. Behavioural adverse effects of KOPr agonists........................................... 38

1.5.5.1. Sedation and psychotomimic effects................................................... 38

1.5.5.2. Depression and aversion ..................................................................... 38

1.5.5.3. Nucleus accumbens and KOPr agonists induced depression and aversion ................................................................................................. 39

1.5.5.4. Central 5HT systems and KOPr activation induced depression and aversion ........................................................................................... 40

1.5.6. Effect of acute vs. chronic kappa opioid receptor activation..................... 42

1.6. Salvinorin A .................................................................................................... 45

1.6.1. Behavioural pharmacology of Salvinorin A................................................ 46

1.6.1.1. Sedation, hallucinations and motor suppression ............................... 46

1.6.1.2. Antinociception ....................................................................................... 46

1.6.1.3. Drug discrimination .................................................................................. 47

1.6.1.4. Anti-cocaine effects ................................................................................ 47

1.6.1.5. Depression and aversion ........................................................................ 48

1.6.1.6. Toxicity studies ..................................................................................... 48

1.6.2. C-2 derived Salvinorin A analogues ............................................................ 51

1.7. Mixed opioid compounds as anti-cocaine agents............................................... 53

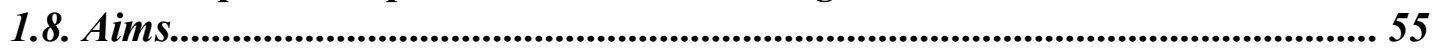




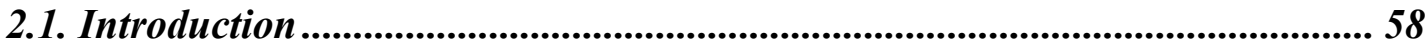

2.1.1. Cocaine prime induced reinstatement model ........................................ 58

2.1.2. Cocaine induced enhancement in motor activity ..................................... 59

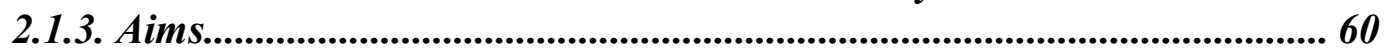

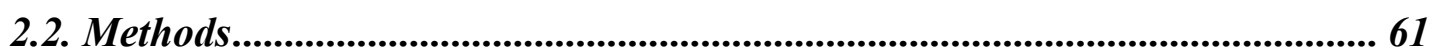

2.2.1. Subjects .............................................................................................................. 61

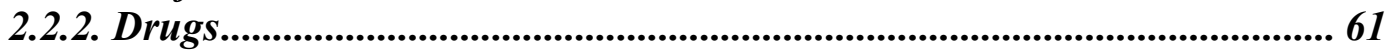

2.2.3. Cocaine prime induced reinstatement test............................................ 62

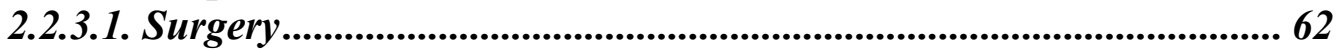

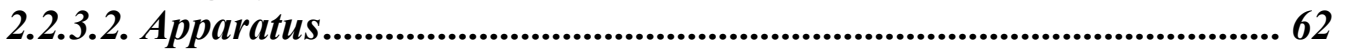

2.2.3.3. Cocaine self-administration training................................................. 63

2.2.3.4. Cocaine prime induced reinstatement test....................................... 63

2.2.3.5. Kappa opioid receptor antagonist reversal experiment.....................65 65

2.2.4. Cocaine induced hyperactivity and behavioural sensitization.................. 67

2.2.4.1. Apparatus................................................................................... 67

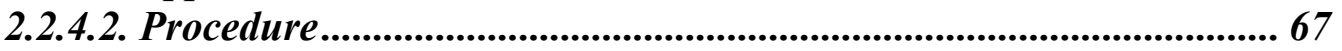

2.2.5. Statistical analysis ............................................................................... 70

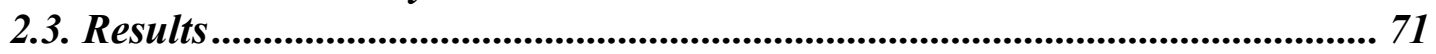

2.3.1. Effect of salvinorin $A$ on cocaine induced reinstatement........................ 71

2.3.2. Effect of salvinorin $A$ on cocaine induced hyperactivity (drug naive rats) .................................................................................................. 73

2.3.3. Effect of salvinorin A on expression of cocaine sensitization ................. 75

2.3.4. Preliminary study to determine pre-treatment time for DS1 .................... 77

2.3.5. Effect of DS1 on cocaine prime induced reinstatement........................... 79

2.3.6. Effect of DS1 on cocaine induced hyperactivity (drug naive rats)........... 81

2.3.7. Effect of DS1 on expression of cocaine sensitization .............................. 83

2.3.8. Effect of MOM Sal B and EOM Sal B on cocaine prime induced reinstatement ............................................................................ 85

2.3.9. Effect of herkinorin on cocaine prime induced reinstatement................. 87

2.3.10. Effect of nalmefene on cocaine prime induced reinstatement ............... 89

2.4. Summary of results................................................................................................ 91

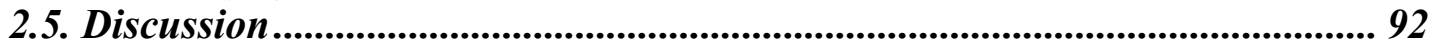

2.6. Summary and conclusions ..................................................................... 101

Chapter 3. Side effect profile of novel kappa opioid receptor agonists .............. 103

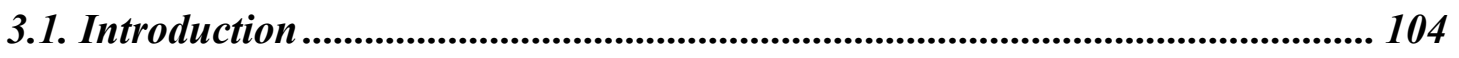

3.1.1. Adverse effects of traditional kappa opioid receptor agonists .................. 104

3.1.2. Pre-clinical models to screen side effect profile of KOPr agonists........... 104

3.1.3. KOPr mediated SERT modulation.......................................................... 105

3.1.4. Live cell ASP${ }^{+}$uptake accumulation model.............................................. 106

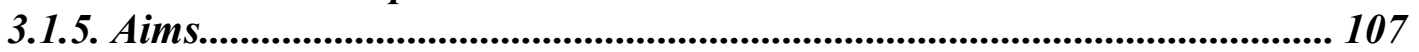

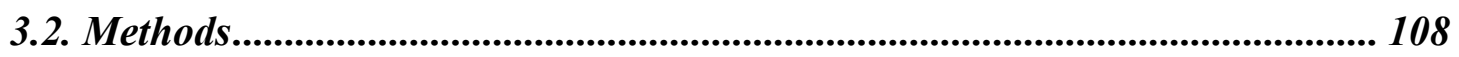

3.2.1. Subjects ................................................................................................ 108

3.2.2. Cell culture maintenance for live cell $A S P^{+}$uptake experiments............. 111

3.2.3. Drugs................................................................................................................. 111

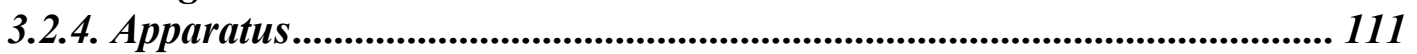

3.2.4.1. Locomotion test................................................................................... 111

3.2.4.2. Sucrose reinforcement................................................................ 111 


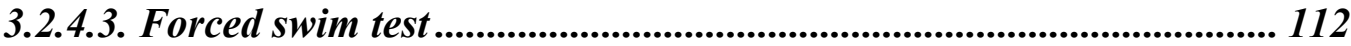

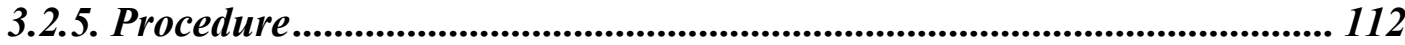

3.2.5.1. Cocaine induced hyperactivity (in self-administering rats).............. 112

3.2.5.2. Spontaneous open field test (drug naive rats) ..................................... 113

3.2.5.3. Sucrose reinforcement training and test ........................................ 113

3.2.5.4. Conditioned taste aversion ..................................................................... 114

3.2.5.5. Forced swim test in drug naive rats and preliminary

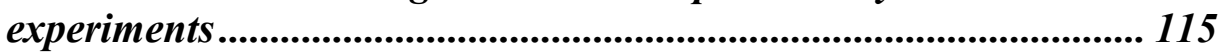

3.2.5.6. Effect of serotonin transporter blockade by fluoxetine on kappa opioid agonists induced depression ...................................... 116

3.2.5.7. Transfections and live-cell imaging to determine SERT function modulation by KOPr agonists (between cell designs) ...................... 116

3.2.6. Data analysis.......................................................................................... 120

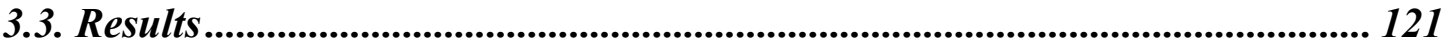

3.3.1. Effect of kappa opioid receptor compounds on cocaine induced hyperactivity (self-administering rats)............................................ 121

3.3.2. Effect of kappa opioid receptor compounds on natural reward reinforcements...................................................................................... 123

3.3.3. Effect of kappa opioid receptor compounds on spontaneous open field activity

3.3.4. Effect of kappa opioid receptor compounds on conditioned taste aversion

3.3.5. Effect of kappa opioid receptor compounds on forced swim test in rats with previous history of cocaine self-administration

3.3.6. Effect of kappa opioid receptor compounds in forced swim test in drug naive rats.

3.3.7. Effect of serotonin transporter blockade by fluoxetine on kappa opioid agonist induced depression in rats.................................................. 133

3.3.8. Control experiments for ASP ${ }^{+}$uptake....................................................... 135

3.3.9. Effect of KOPr agonists pre-treatment on ASP ${ }^{+}$uptake ............................ 137

3.4. Summary of results........................................................................................ 139

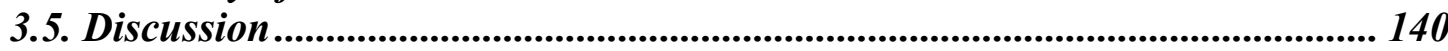

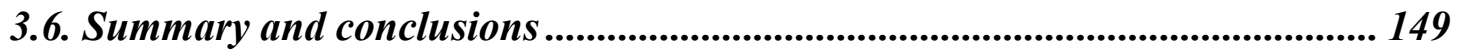

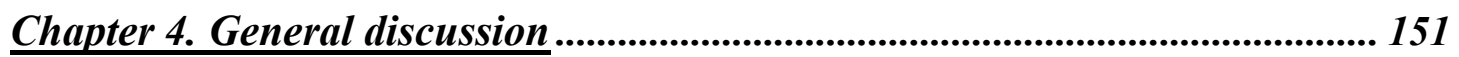

4.1. Endogenous KOPr systems and cocaine addiction ........................................ 152

4.2. Limitations of classical KOPr agonists: rationale for current thesis.............. 156

4.3. Pharmacological profiling of novel KOPr agonists.......................................... 157

4.4. Significance of present results.................................................................... 162

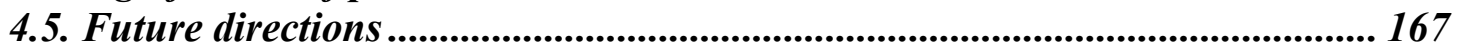

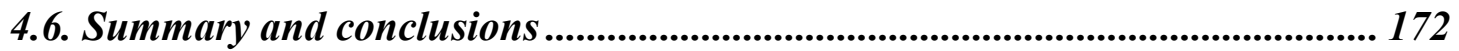

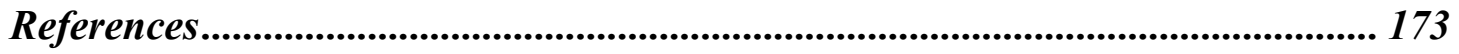

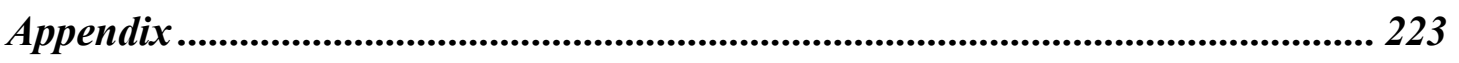




\section{$\underline{\text { Abstract }}$}

$\underline{\text { Rationale }}$ Kappa opioid receptor (KOPr) activation by traditional agonists has been shown to produce anti-addiction behaviours. However, adverse effects such as sedation, aversion and depression have limited their clinical development. Recently, salvinorin A (Sal A), an active component of the plant Salvia divinorum was shown to be a potent and selective KOPr agonist. Sal A has a short duration of effect and quick onset of action. It also produces similar behavioural pharmacology to traditional KOPr agonists. However, little is known about the anti-addiction profile of Sal A. If Sal A and its structural analogues produce anti-addiction properties with fewer adverse effects compared to traditional KOPr agonists, they have potential to be developed into antiaddiction pharmacotherapies. Therefore, Sal A and its structural analogues (DS1, MOM Sal B, EOM Sal B, herkinorin) and Mu opioid receptor (MOPr) antagonist/partial KOPr agonist, nalmefene were tested for their behavioural anti-addiction and adverse effect profiles in rats.

Methods To test the anti-addiction profile, a within session cocaine prime induced reinstatement paradigm was used. The selectivity of KOPr agonists in attenuating cocaine seeking behaviours was tested using sucrose reinforcement (anhedonia) and cocaine induced hyperactivity in self-administering rats (sedation during reinstatement test). Furthermore, behavioural adverse effects were screened using spontaneous open field activity (motor suppression), conditioned taste aversion (aversion) and forced swim test (depression) in rats. To further quantify the anti-addiction behaviours, the effect of KOPr agonists which attenuated drug seeking selectively without producing motor suppression by themselves were tested for cocaine produced motor function (hyperactivity and behavioural sensitization) in rats. The effect of serotonin transporter blockade on KOPr agonist induced depressive behaviour was also tested. The effects of KOPr activation on in vitro serotonin transporter function were also determined. 
$\underline{\text { Results }}$ Sal A, DS1 and nalmefene attenuated cocaine prime induced drug-seeking, in a selective manner, via KOPr activation. MOM Sal B, a more potent and long acting Sal A analogue attenuated cocaine seeking in a non-selective manner. Sal A, DS1 and nalmefene did not induce aversion, however nalmefene suppressed motor function, which was not seen with Sal A and DS1. Furthermore, Sal A and DS1 suppressed cocaine behavioural sensitization. All three compounds (Sal A, DS1, nalmefene) produced depression. The depressive effects produced by Sal A and DS1 were diminished by blocking the serotonin transporter. Live-cell serotonin transporter assays showed potential differences between traditional (U50488H) and novel (Sal A, DS1) KOPr agonists in their ability to modulate serotonin transporter function.

Conclusion Out of six KOPr compounds tested, Sal A, DS1, MOM Sal B and nalmefene produced anti-addiction behaviours. However, MOM Sal B exposure also suppressed natural reward seeking behaviour. Sal A and DS1 had a better adverse effect profile than nalmefene. Thus, the order of efficacy for the compounds tested were DS1 $\geq$ Sal A $>$ nalmefene $>$ MOM Sal B. However depression was noted with all three compounds tested (Sal A, DS1, nalmefene) and our study provides evidence to suggest the involvement of the serotonin system in Sal A and DS1 induced depression. Moreover, a difference in modulation of serotonin transporter function by novel and traditional KOPr agonists was observed. 


\section{Acknowledgements}

Work of this nature cannot be achieved without support from so many fronts and here I take the opportunity to remember and thank those peoples who helped me fulfil this dream into reality.

To start with, I thank my spiritual paramguru, Mawla Ali (a.s.) for giving me strength, passion, positive energy and clarity of mind which has helped and guided me throughout my life. I thank my lovely father for his love, compassion, understanding and patience. I thank my mother, for her love, prayers and hard work during my early years of upbringing which has helped me shape into the person I am today. I take an opportunity to remember my dearest grandmother for every wonderful thing she did for me.

I would thank my supervisor, Dr. Bronwyn Kivell for her support, help, encouragement and efforts in sharpening my writing and presentation skills. I also thank my cosupervisor Prof. Susan Schenk for her assistance and encouragement in getting this work done successfully. I thank Dr. Thomas E. Prisinzano for providing samples of salvinorin A and its analogues. I also thank Dr's Samanda Ramamoorthy and Lakshmi Devi for their generous gift of plasmids for human serotonin transporter and rat kappa opioid receptor respectively. I am grateful to Prof. Subhash L. Bodhankar for introducing me to behavioural pharmacology.

Big thanks to Mr. Richard Moore, for his wonderful assistance with a gentle sense of humour. Also, thanks to Ms. Sushila Pillai for training me and troubleshooting the confocal microscope problems. I thank Mr. Alex Howard for training me with selfadministration experiments, Mr. Caleb Carati and Mr. Alex Crowther for doing surgery on my rats, Dr. Lincoln Hely for helping in sucrose reinforcement experiments and Mr. Neville Higgison, Mr. Cameron Jack and Mr. Craig Doney for their help in setting up the forced swim test in our lab. I also thank all the wonderful people from the Psych rat lab for their support.

Special thanks to Ms Bridget Simonson and Ms. Kirsty Danielson for training me in tissue culture, listening to my presentations and for going through my thesis manuscript. I also thank all the lovely people from KK601 lab for their support. I thank the wonderful administrative support staff of SBS, especially Ms Sandra Taylor for making those hard times easy with her cheerfulness. 
I thank the Neurological Foundation of NZ, Health Research Council, Wellington Medical Research Foundation, Victoria University of Wellington and the Victoria University $\mathrm{PhD}$ Scholarship for funding this study.

Finally, I am indebted to the animals whose lives were sacrificed for this noble cause.

Aashish S. Morani. 


\section{List of Figures}

Fig.1.1. Stages in the cycle of addiction ....................................................................... 19

Fig.1.2. Effect of cocaine on dopamine transporter function .............................. 26

Fig.1.3. Signal transduction mechanism for kappa opioid receptor activation..... 28

Fig.1.4. Negative feedback loop ........................................................................... 30

Fig.1.5. Kappa opioid receptor agonists ............................................................... 32

Fig.1.6. Salvinorin A, its analogues and nalmefene ............................................. 52

Fig.2.1. Cocaine prime induced reinstatement test ................................................ 66

Fig.2.2. Effect of Sal A on cocaine prime induced reinstatement........................ 72

Fig.2.3. Effect of Sal A on cocaine induced hyperactivity (drug naive rats) ......... 74

Fig.2.4. Effect of Sal A on expression of cocaine sensitization and stereotypy..... 76

Fig.2.5. Preliminary study to determine pre-treatment time for DS1.................... 78

Fig.2.6. Effect of DS1 on cocaine prime induced reinstatement........................... 80

Fig.2.7. Effect of DS1 on cocaine induced hyperactivity (drug naïve rats) ........... 82

Fig.2.8. Effect of DS1 on expression of cocaine sensitization and stereotypy....... 84

Fig.2.9. Effect of MOM Sal B and EOM Sal B on cocaine prime induced reinstatement 86

Fig.2.10. Effect of herkinorin on cocaine prime induced reinstatement ............... 88

Fig.2.11. Effect of nalmefene on cocaine prime induced reinstatement................ 90

Fig.3.1. ASP ${ }^{+}$accumulation and fluorescence analysis......................................... 119

Fig.3.2. Effect of KOPr compounds on cocaine induced hyperactivity

(cocaine self-administering rats) ...................................................................... 122

Fig.3.3. Effect of KOPr compounds on natural reward reinforcement ................ 124

Fig.3.4. Effect of KOPr compounds on motor function .......................................... 126

Fig.3.5. Effect of KOPr compounds on conditioned taste aversion ...................... 128

Fig.3.6. Effect of KOPr compounds on FST in rats with previous history of cocaine self-administration....................................................................... 130

Fig.3.7. Effect of KOPr compounds on FST in drug naive rats............................. 132

Fig.3.8. Effect of SERT blockade by FLX on KOPr agonists produced depression in FST ............................................................................................ 134

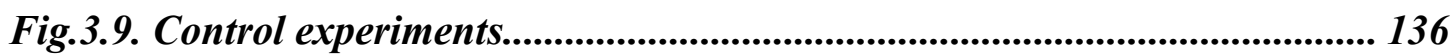

Fig.3.10. Effect of KOPr agonists pre-treatment on SERT function .................... 138

Fig.4.1. KOPr system modulation in addiction cycle ................................................. 155

Fig.4.2. Pharmacological profiling of novel KOPr agonists ................................... 160 


\section{List of Tables}

Tab.1.1.Stage of addiction and corresponding pre-clinical paradigms.................. 22

Tab.1.2. KOPr agonists in pre-clinical studies of addiction ................................. 37

Tab.1.3. Salvinorin A: Pre-clinical studies.............................................................5 50

Tab.2.1. Experimental groups for cocaine induced hyperactivity......................... 69

Tab.2.2. Experimental groups for cocaine behavioural sensitization .................... 69

Tab.2.3.Effect of KOPr agonists on cocaine prime induced drug-seeking ............ 91

Tab.2.4. Effect of KOPr agonists on cocaine induced motor activity.................... 91

Tab.3.1. Amount of cocaine consumed by rats used for preliminary

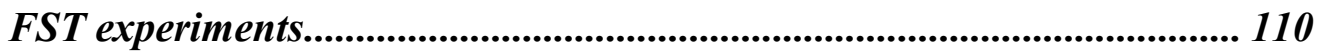

Tab.3.2. Control experiments to determine selectivity of KOPr agonists in attenuating reinstatement.......................................................................... 139

Tab.3.3. Adverse effect profile of novel KOPr agonists ........................................ 139

Tab.3.4.Effect of serotonin transporter blockade on KOPr agonists

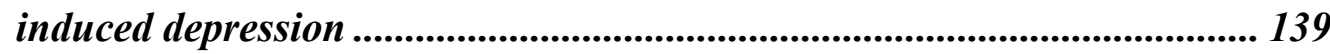

Tab.3.5. Effect on SERT modulation by traditional and novel KOPr agonists using live cell ASP+ uptake....................................................................... 139

Tab.4.1. Pharmacological screening of novel KOPr agonists............................... 161

Tab.4.2. Binding affinities and efficacy for KOPr and its effect on cocaine-seeking behaviour 166 


\section{List of abbreviations}

\begin{tabular}{ll} 
5HT & - serotonin \\
ASP & - 4-(4-(dimethylamino)-styryl)-N-methylpyridinium \\
cAMP & - cyclic adenosine mono-phosphate \\
CPP & - conditioned place preference. \\
CPU & - caudate putamen \\
CREB & - cyclic AMP response element binding protein \\
CTA & - conditioned taste aversion \\
DA & - dopamine \\
DAT & - dopamine transporter \\
DS1 & - 2-mesylate salvinorin B \\
DYN & - dynorphin \\
EOM Sal B & - ethoxymethyl salvinorin B \\
FLX & - fluoxetine \\
FR-1 & - fixed ratio 1 schedule of reinforcement \\
FR-5 & - fixed ratio 5 schedule of reinforcement \\
FST & - forced swim test \\
GFP-hSERT & - green fluorescence protein tagged human serotonin transporter. \\
HEK-293 & - human embryonic kidney cells \\
ICSS & - intracranial self-stimulation \\
icv & - intracerebroventricular \\
ip & - intraperitoneal \\
iv & - intraveneous \\
KOPr & - Kappa opioid receptors \\
KRH & - Krebs Ringer Herpes buffer \\
MDS & - mesocorticolimbic dopamine system \\
MOM Sal B & - methoxy-methyl salvinorin B \\
MOPr & - Mu opioid receptors \\
myc-rKOPr & - myc tagged rat kappa opioid receptor. \\
NAc & - nucleus accumbens \\
NET & - nor-epinephrine transporter \\
Nor-BNI & - norbinaltorphimine \\
PFC & - pre-frontal cortex \\
RDEV & - rotating disc electrode voltammetry \\
Sal A & - Salvinorin A \\
Sal B & - salvinorin B \\
sc & - subcutaneous \\
SERT & - serotonin transporter \\
SSRI & - selective serotonin reuptake inhibitor \\
VTA & - ventral tegmental area \\
\hline ing
\end{tabular}


Chapter 1. General Introduction 
Addiction is defined as "compulsive physiological need for and use of a habitforming substance (as heroin, nicotine, or alcohol) characterized by tolerance and by well-defined physiological symptoms upon withdrawal; broadly : persistent compulsive use of a substance known by the user to be physically, psychologically, or socially harmful" (addiction. 2011. In Merriam-Webster.com). Drugs of abuse, such as psychostimulants produce a general sense of euphoria or hedonism which determines their abuse potential (Eddy et al., 1965; Koob and Le Moal, 1997). Initial exposures to these compounds produce strong reinforcing properties which have been characterised as a sense of well-being (Koob and Bloom, 1988; Gawin, 1991). The reinforcing properties change the complexion of normal brain function which is evident by the alteration in normal brain anatomy, biochemistry and underlying molecular and cell biology (Verdejo-Garcia et al., 2007; Nestler and Aghajanian, 1997; Wexler et al., 2001; Chang and Chronicle, 2007; Koob and Le Moal, 2005). These alterations are responsible for withdrawal effects such as anxiety, depression and stress, which predispose the addict to relapse back to the use of these drugs of abuse (Eddy et al., 1965; Sinha, 2001; Van Bockstaele et al., 2010; Valentino et al., 2010).

Apart from alcohol and nicotine use, commonly abused illegal drugs include psychostimulants (cocaine, amphetamine, methamphetamine), opioids (heroin, morphine) and recreational hallucinogens (lysergic acid diethylamide, psilocybin, cannabis) (Substance Abuse and Mental Health Services Administration 2008; World Drug Report, 2007). Among these illegal drugs of abuse, cannabis is most abused in The United States, Europe, Australia and New Zealand (World Drug Report, 2007; Substance Abuse and Mental Health Services Administration 2008; Australian Drug and Alcohol Use Survey 2007; New Zealand Alcohol and Drug Use Survey, 2010). The next most abused illegal drug in Europe and the United States is cocaine (Substance Abuse and Mental Health Services Administration 2008; The state of drug problem in 
Europe, Annual report 2009). In New Zealand, around half of the population between 16-64 years of age had abused illegal drugs (excluding alcohol, nicotine and party pills) at least once in their lifetime. Moreover, the prevalence of psychostimulant use was comparatively lower than cannabis use, with fewer than $4 \%$ of the surveyed population abusing psychostimulants (cocaine, methamphetamine and prescription stimulants) as compared to $46.4 \%$ using cannabis (New Zealand Alcohol and Drug Use Survey, 2010). These surveys indicate that use of illegal drugs is widespread.

Substance abuse is associated with social, economic and civic disabilities (New Zealand Alcohol and Drug Use Survey, 2010; World Drug Report, 2007). The societal effects of substance use include loss in school hours, work hours, and unemployment (New Zealand Alcohol and Drug Use Survey, 2010). Moreover, increased crime and violence burdens healthcare and justice resources and add to the social and economic cost associated with substance abuse (World Drug Report, 2007). Furthermore, drug abuse contributes to an increase in co-morbid mental illnesses and infectious diseases such as acquired immune deficiency syndrome and hepatitis $\mathrm{C}$, which are mainly caused by drug users sharing needles (Morton, 1999; Murrill et al., 2001; Sorenson et al., 2002). In 2008, the financial loss associated with substance abuse in the United Kingdom was over 20 billion pounds (The state of drug problem in Europe, Annual report 2009). Due to the large social and economic cost of addiction many strategies, such as strict law enforcement have been undertaken to control this menace (World Drug report 2007). From a medical perspective, one of the ways to tackle this problem is to develop pharmacological approaches to reduce or prevent addiction.

Numerous studies have been conducted to determine how drugs of abuse bring about the long lasting changes in brain physiology (Weiss et al., 1992; Pierce and Kalivas, 1997; Koob et al., 2004; Koob and Le Moal, 2008; Koob and Volkow, 2009). It is well accepted that drugs of abuse function by modulating the mesocorticolimbic 
dopamine system (MDS; Di Chiara and Imparato, 1988a; Wise and Bozarth, 1984; Wise and Rompre, 1998; Kuhar et al., 1991; Spanagel et al., 1992; Koob, 1992). The MDS comprises of dopamine (DA) cell bodies located in the ventral tegmental area (VTA) and projections to the nucleus accumbens (NAc), amygdala and the pre-frontal cortex (PFC) (Olds and Milner, 1954; Olds, 1956, 1977; Bozarth, 1987). Almost all drugs of abuse act by increasing the DA levels in the MDS (Wise and Rompre, 1989). This finding formed the basis of the 'dopamine theory of addiction', which links the increase in reinforcing properties of the drugs of abuse to the elevated DA levels in the MDS (Wise and Bozarth, 1984; Wise and Rompre, 1989; Kuhar et al., 1991; Spanagel and Weiss, 1999). However, recent evidence also suggests that DA plays a role in craving, incentive learning for drug induced enhancement in reward experience and its sensitization (Robinson and Berridge, 1993; 2003; Di Chiara, 1995; Lambert et al., 2006). Long term use of drugs of abuse alters the normal reward and motivational behaviour by bringing alterations in normal reward circuitry (Weiss et al., 1992; Spanagel et al., 1994; Nestler et al., 2001). These long term neuronal adaptations can lead to withdrawal related behaviours such as depression and stress, which are implicated in relapsing back into substance use (Bruchas et al., 2010; Nestler et al., 2001). In addition to their effect on the reward pathway, drugs of abuse also bring about changes in brain regions involved in memory processing, decision making and learning novel tasks (Grant et al., 2000; Schneider et al., 2008; Schenk et al., 2010). The following section will describe various therapeutic strategies applied to tackle addiction at the clinical level. 


\subsection{Current pharmacotherapies for addiction}

Addiction is a chronic relapsing disorder; therefore treatment strategies involve multiple behavioural and/or pharmacological interventions with intensive patient monitoring (DeRubeis and Crits-Christoph, 1998; Carrol and Enken, 2005). This makes for long term patient management rather than short term therapy (McLellan et al., 2000). Although, treatment choice varies between individuals, most treatment strategies include cognitive behavioural therapy in combination with pharmacological agents (Jupp and Lawrence, 2010). Behavioural therapy includes group counselling, social involvement (family and friends) and motivational therapy to enhance self-abstinence from drug taking (Carrol and Enken, 2005; Carrol et al., 2006). The role of pharmacological treatment is to tackle the patho-physiological alterations caused by the abused drugs, and to prevent the addict from relapsing back to drug use (Jupp and Lawrence, 2010; Lobmaier et al., 2010).

This section discusses some currently available pharmacotherapeutic strategies for drug of abuse including opioids, alcohol, nicotine and psychostimulant abuse. Opiate maintenance or replacement therapy for heroin addiction aims to reduce withdrawal related adverse effects such as depression, craving and preventing the abuser from relapsing back to drug use (Kreek et al., 2000; 2005). This therapeutic approach uses MOPr agonists such as methadone and methadone derivatives which reduce the craving effect of heroin addiction (Stimmel and Kreek, 2000). Because methadone is given via an oral route, the "high" produced by methadone is much less than heroin (Hickman et al., 2003; Lobmaier et al., 2010). This may account for its overuse and potentially fatal toxicity (Perret et al., 2000; Reingardiene et al., 2009). Recently, buprenorphine, a mixed opioid compound (MOPr agonist/antagonist and partial KOPr antagonist) has also been used in the opioid maintenance therapy (Soyka et al., 2008). 
However, in clinical settings, methadone is still more widely used over buprenorphine (Mattick et al., 2008).

Similar to opiates, nicotine replacement therapy has been applied to manage the withdrawal effects associated with smoking cessation (Silagy et al., 2004). The principle behind this is to make nicotine available via delivery systems other than cigarette smoke such as nicotine patches, inhalers, chewing gums and lozenges (Silagy et al., 2004). However, high rates in relapse back to smoking have been noted within a short period with nicotine replacement therapy (Borgne et al., 2004). Thus, nicotine replacement therapy seems to fail in providing long term abstinence from quitting smoking. Other pharmacological approaches include the use of partial $\alpha 4 \beta 2$ nicotinic agonists, varenicline and bupropion (nicotinic receptor blocker and DA/nor-epinephrine (NE) neurotransmission modulator) (Jorenby et al., 2006). Both of these agents increase the period of abstinence (Jorenby et al., 2006) by decreasing craving and withdrawal effects (Coe et al., 2005). However, recent reports suggest the development of depression and suicidal tendencies with these pharmacotherapies (Gunnel et al., 2009; Wrightman et al., 2010).

Modulation of drug metabolism is often used in the treatment of alcohol addiction and disulfiram, via inhibiting enzyme acetaldehyde dehydrogenase has been used to prevent alcohol intake (Kitson, 1977). It produces acute aversive symptoms such as flushing, irritability, tachycardia, nausea and vomiting if alcohol is ingested, thus, preventing the abuser from consuming alcohol (Kitson et al., 1977). Although there are compliance issues, this treatment has been successfully applied in clinical settings under medical supervision (Ehrenreich and Krampe, 2004).

There are currently no successful pharmacotherapies to treat psychostimulant addiction (Taylor and Gold, 1990; Gawin, 1991). The cocaine analogue, cocaethylene 
has been tested for use in cocaine withdrawal and maintenance therapy. Cocaethylene was effective in decreasing behavioural effects of cocaine in humans (Baker et al., 2007). However, due to its toxic liability, it is likely to fail as anti-cocaine agents (Tacker and Okorodudu, 2004). Monoamine oxidase inhibitors such as selegiline have shown promising anti-cocaine effects in pre-clinical studies but have failed to produce similar effects in humans (Elkashef et al., 2006; Gatch et al., 2006). Additionally, Negus and Mello, (2004) showed that methadone infusions $(0.032-1.0 \mathrm{mg} / \mathrm{kg} / \mathrm{hr})$ produced non-specific decrease in operant responding without affecting cocaine reinforcements in rhesus monkeys.

These reports show that most of the currently available pharmacotherapies have shown limited success. In some cases drug-addicts have a high incidence of relapse and risk fatal drug induced toxicity. Furthermore, some of these medications can themselves cause addiction (methadone for opioid maintenance therapy), or have safety and compliance issues warranting caution while being used clinically (disulfiram, varenicline, bupropion). The development of more effective anti-addiction pharmacotherapies are therefore needed to help break the addiction cycle and to reduce relapse. Understanding the eiteology of addiction cycle can help in designing new treatment strategies which will be useful to combat the progression and development of substance abuse (Markou et al., 1993; Le Moal and Koob, 2005). 


\subsection{The addiction cycle}

The development of addiction cycle has been extensively reviewed and has been studied both pre-clinically and clinically (Gawin and Kleber, 1988; Koob, 2008, Koob and Le Moal, 2008; Koob and Volkov, 2009). Briefly, the addiction cycle consists of three different stages, the initial binging stage, followed by drug abstinence and relapse (Fig 1.1; Koob and La Moal, 2008). The binging phase is characterized by the hedonic properties of the drugs of abuse (Gawin, 1991). During this stage the drug-taker experiences positive reinforcement (A in Fig. 1.1; Eddy et al., 1965; Gawin, 1991) and this leads to a gradual escalation in drug use (B in Fig. 1.1). During the abstinence from drug use (forced or self-imposed; shown as C in Fig 1.1), neuronal adaptations occurring especially in the brain reward circuits results into negative mood states such as dysphoria, stress and depression (Koob and Volkov, 2009; Carlezon et al., 1998; Nestler, 2001; D in Fig. 1.1). These negative mood states during abstinence can enhance drug craving (E in Fig. 1.1) and re-exposure to a drug prime or an environmental cue associated with drug use (such as party settings where drugs were abused) or stress can predispose relapse (Hunt et al., 1971; Goeders, 2002a; 2002b). Pre-clinical models of addiction have been shown to mimic the behavioural conditions observed in humans at different stages of the addiction cycle (Markau et al., 1993; Koob, 2000). The following section will discuss various animal models commonly applied in addiction research and anti-addiction drug discovery. 


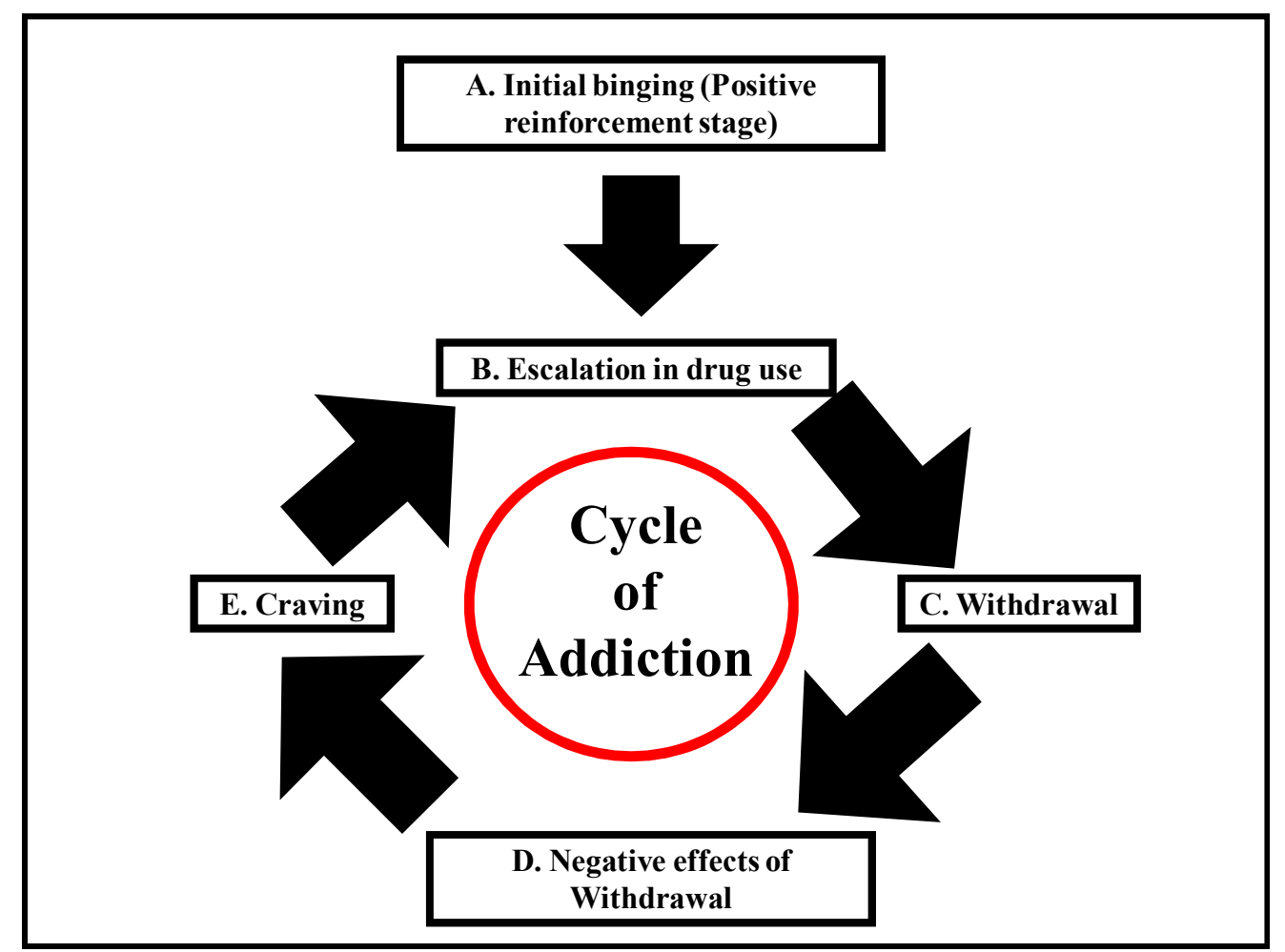

\section{Fig 1.1 Stages in the cycle of addiction}

(Adapted from Koob and Le Moal, 2008). After initial 'binges' of drug exposure (A), escalation in the drug administration develops (B). This is due to the positive reinforcing or hedonic effects of drugs of abuse. Later on during abstinence from the drug use (C), negative mood states such as depression and stress are observed (D). These negative states are due to the neuronal adaptations associated with drug use and can lead to craving (E). Exposure to drug, drug associated environmental cues and stressors can predispose the addict to relapse back to the drug use. This marks the establishment of the addiction cycle. 


\subsection{Animal models of addiction}

Animal models are an important tool which have been utilized to study the underlying pathophysiology of addiction and also to test novel therapeutic strategies (Spanagel, 2003; Self, 2004; Kalivas et al., 2006). The use of various animal models in understanding the stages of the addiction cycle has been reviewed extensively (Auriacombe et al, 1997; Koob et al, 1999; Stewart, 2000; Sanchis-Segura and Spanagel, 2006; and Spanagel and Holter, 2000; Shalev et al., 2002; O’Brian and Gardner, 2005; Kalivas et al, 2006; Koob and Le Moal, 2008; Koob and Le Moal, 2005). Animal models commonly applied to study various stages in addiction cycle are shown in Tab. 1.1.

The positive reinforcement stage has been studied using models which measure intravenous or oral drug self-administration, conditioned place preference $(\mathrm{CPP})$, or intracranial self-stimulation (ICSS). The craving stage can be depicted by models measuring the reinstatement of extinguished drug-seeking and behavioural sensitization. The negative reinforcement stage of the addiction cycle can be depicted in models measuring drug self-administration, ICSS, conditioned place aversion and drug discrimination. (Koob and La Moal, 2005; Sanches-Segura and Spanagel, 2006).

\subsubsection{Operant conditioning in studying addictive behaviours.}

Most of these paradigms are based on the rationale of 'Skinner's box' which applies the principle of 'operant reinforcer induced reward' (Skinner, 1938). Briefly, a reinforcer is any stimulus which is used to learn a task (operant lever pressing) and will lead to a response (delivery of reward such as palatable food or drugs of abuse) (Skinner, 1938; Shalev et al., 2002). The hedonic nature of response will lead to an increased lever pressing, thus leading to positive reinforcement (Skinner, 1938; Dinsmoor, 2004; Shalev et al., 2002). Once the animal learns to obtain reward, the effect of exposure to a pharmacological/ external stimulus can be determined on the positive reinforcement 
(Shaham et al., 2003). Such conditioning is called 'instrumental conditioning' and the pharmacological/external stimuli applied are known as 'unconditioned stimulus' (Thorndike, 1911; Pavlov, 1927; 1941; Dinsmoor, 2004). Drugs of abuse such as cocaine, amphetamine and morphine act as positive reinforcers and have been shown to increase operant responding in laboratory animals (Deaneu et al., 1969; Devine and Wise, 1994). Furthermore, the principle of instrumental conditioning has been applied to understand the craving for abused drug by using the reinstatement model (Stewart, 2000; Shalev et al., 2002; Shaham et al., 2003). By using this model, presentation of drug primes (De Wit and Stewart, 1981; 1983), environmental cues (Wissenborn et al., 1995; Koob et al., 1996) and stressors (Erb et al., 1996) have been shown to reinstate extinguished drug-seeking behaviours in rats. This paradigm has been used extensively to determine the pathophysiology of drug addiction as well as anti-addiction drug discovery (Kalivas et al., 2006; Stewart, 2000; Shalev et al., 2002; Self, 2004).

\subsubsection{Non-operant conditioning in studying aversive behaviours.}

In addition to operant conditioning, laboratory animals can also be trained using non-operant conditioning methods to study the aversive behaviours (Sanchez Segura and Spanagel, 2006). One of the paradigms based on this principle is conditioned taste aversion (CTA). In this test, water deprived animals are presented with a novel tasting saccharin solution. The novel taste of saccharin (conditioned stimuli) is paired with the unconditioned stimuli (x-ray irradiation or pharmacological agents; Garcia et al., 1955; Davis et al., 2009). A reduction in the amount of saccharin consumed when presented next shows the aversive properties produced by the unconditioned stimuli (Sanchez Segura and Spanagel, 2006; Davis and Riley, 2010). 
Tab. 1.1. Stage of addiction and corresponding pre-clinical paradigms.

\begin{tabular}{|l|l|l|}
\hline Addiction stage & $\begin{array}{l}\text { Source of } \\
\text { reinforcement }\end{array}$ & Preclinical models \\
\hline Binging & Positive reinforcement & $\begin{array}{l}\text { Increased self-administration and } \\
\text { CPP, decreased ICSS thresholds }\end{array}$ \\
\hline $\begin{array}{l}\text { Craving/ } \\
\text { anticipation }\end{array}$ & $\begin{array}{l}\text { Conditioned positive } \\
\text { reinforcement }\end{array}$ & $\begin{array}{l}\text { Drug prime, stress and cue } \\
\text { induced reinstatement, } \\
\text { behavioural sensitization }\end{array}$ \\
\hline Withdrawal stage & Negative reinforcement & $\begin{array}{l}\text { Conditioned place aversion, } \\
\text { increased self-administration } \\
\text { (dependence stage), increased } \\
\text { ICSS thresholds }\end{array}$ \\
\hline
\end{tabular}

(Modified from Sanchez-Segura and Spanagel, 2006; Koob and La Moal, 2008). 


\subsection{Cocaine addiction in pre-clinical models.}

Cocaine has been widely used by the indigenous communities in South America for over 3000 years (Siegel, 1982; Gawin and Kleber, 1988). Its addictive potential has been extensively studied in pre-clinical as well as clinical settings (Shaham et al., 2000; Sughondhabirom et al., 2005). Cocaine acts as a positive reinforcer and has been shown to produce self-administration in rats, mice and monkeys (Pickins and Thompson, 1968; Deneau et al., 1969; Hill and Powell, 1976; Papasava et al., 1981). It also produces discriminative stimulus effects (Spealman et al., 1991), CPP (Shippenberg et al., 1995) and decreases ICSS thresholds (Markou and Koob, 1991; Kornetsky and Esposito, 1981) in laboratory animals. A single injection of cocaine has also been shown to enhance motor function (Bhattacharya and Pradhan, 1979; Kalivas and Duffy, 1990). When cocaine is either experimenter- (Kalivas and Duffy, 1990; Kalivas and Stewart 1991; Heidbreder et al., 1996; Kalivas et al., 1998) or self-administered (Hooks et al., 1994; Philips and Di Ciano, 1996), it produces sensitization to its motor stimulant effects. Cocaine has been shown to reinstate extinguished drug-seeking behaviour following drug primes (Downs and Woods, 1974; de Wit and Stewart, 1981; 1983; Worley et al., 1994; Stewart, 2000; McFarland and Kalivas, 2001). Additionally, presentation of environmental cues associated with cocaine self-administration (Koob et al., 1995; Weissenborn et al., 1996) and environmental stressors have also been shown to induce reinstatement of cocaine seeking behaviour (Erb et al., 1996; Kupferschmidt et al., 2011). The following sections will briefly describe the mechanism by which cocaine induces addictive behaviours.

\subsubsection{Effect of cocaine on DA transporter function}

Synaptic DA neurotransmission following cocaine use is shown in Fig 1.2A. On activation, the pre-synaptic neurons release DA which binds to DA receptors and depending on the receptor subtypes it activates, can produce neuronal excitation (Fig 
1.2B). Later, the released DA from the receptors gets transported back to the presynaptic neurons via the DA transporter (DAT). There it is either repackaged into vesicles for re-release or degraded by enzymes (Carlsson, 1969; Vogt, 1969; White, 1990).

The transportation of DA into the pre-synaptic neuron requires sodium and chloride ions to bind to DAT (Zhanisar and Dolen, 2001). This is followed by DA binding which leads to the structural conformation change in DAT, resulting in DA uptake into the neuron (Torres et al., 2003). This process requires de-phosphorylation of adenosine tri-phosphate to adenosine di-phosphate and is facilitated in the presence of the enzyme $\mathrm{Na}^{+} / \mathrm{K}^{+}$ATPase (Fig 1.2C; Sonders et al., 1997). Along with DAT, cocaine is also a substrate for the nor-epinephrine transporter (NET) and serotonin transporter (SERT) (Ravna et al., 2003). Its action on the reward circuit is mediated mainly via its interaction with DAT (Kuhar et al., 1991). Recent studies have shown that cocaine and DA bind at the same site on DAT (Beuming et al., 2008). The increased binding of cocaine to DAT results into decreased DA uptake and increased availability of DA in the synapse (Fig 1.2D; Zhaniser et al., 1999). The rewarding effects of cocaine are due to its modulation of DA neurotransmission, thereby acting as an indirect DA receptor agonist (Kreek et al., 1999; Zhaniser and Dolen, 2001; Torres et al., 2003).

Due to this, pharmacological agents which modulate DA receptors have been studied as anti-cocaine pharmacotherapeutics (Jupp and Lawrence, 2010; Heidbreder, 2008). However, studies have indicated that DA receptor antagonists produce adverse effects such as suppression of reward reinforcements and extra-pyramidal motor effects which have discouraged their development as anti-cocaine agents (Woolverton et al., 1986; Wise and Schwartz, 1981; Mello and Negus, 1996). Recent studies have shown that the DA 3 receptor preferring compound ropinirole has an anti-addictive effect in humans (Meini et al., 2008). The partial DA 2 receptor agonist, aripiprazole has also 
been shown to decrease cocaine craving (Vorspan et al., 2008). On the other hand, the DA 1/DA 2 receptor agonist amantadine showed no significant effect in treating cocaine dependence (Kampman et al., 2006). Furthermore, recent in vitro studies have shown that DA 2 receptor activation has shown to increase DAT function (Bolan et al., 2007). Taken together, these results indicate that modulating DA 2 receptor tone could be useful in maintaining cocaine withdrawal effects. However, serious adverse effects such as potentiating other drug use (aripiprazole potentiating methamphetamine and amphetamine use) can be a limiting factor in developing these agents as anti-cocaine pharmacotherapies (Tiihonen et al., 2007). Recently, benztropane DAT inhibitors such as JHW 007 (Velazquez-Sanchez et al., 2010) and AHN 1055 (Velazquez-Sanchez et al., 2009; Ferragud et al., 2009) have been tested as cocaine replacement agents in animals. Results from these studies showed that DAT antagonism may prevent cocaine relapse. This, however, needs to be tested in humans to determine its clinical application. Collectively taken, these results strongly suggest developing agents which indirectly modulate DA neurotransmission, ideally with fewer adverse effects may be an effective strategy to tackle cocaine addiction, (Sonders et al., 1997; Mello and Negus, 2000).

It is well documented that KOPr activation decreases DA levels in NAc (Di Chiara and Imparato, 1988a; 1988b; Spanagel et al., 1992). This effect produced by KOPr agonists might be due to an increase in DAT function (Thompson et al., 2000) as both KOPr and DAT are co-localized in NAc (Svingos et al., 2001). Furthermore, preclinical studies have shown that KOPr activation opposes cocaine produced behaviours (Heidbreder et al., 1993; Shippenberg et al., 1998). Therefore, KOPr activation can indirectly modulate cocaine produced behavioural and neurochemical alterations (Thompson et al., 2000). Thus, making them an ideal candidate to be developed as anticocaine agents (Prisinzano et al., 2005; Shippenberg et al., 2001). 

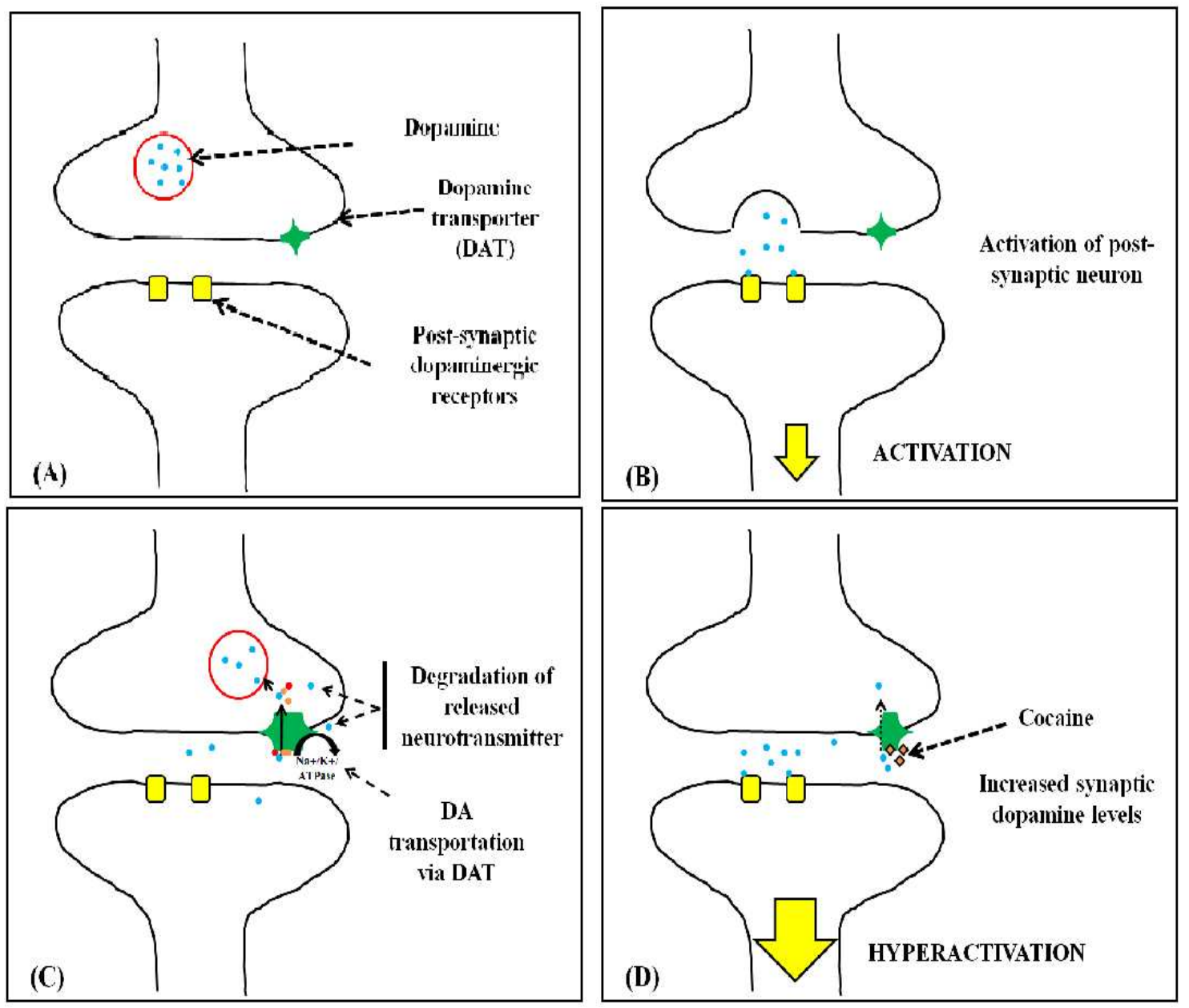

\section{Fig.1.2 Effect of cocaine on dopamine transporter function}

Schematic representation of the effect of cocaine on DA neurotransmission. A. Pre-synaptic neuron and a post- synaptic neuron. The pre-synaptic neuron has stored dopamine (DA) in the vesicles and DAT is present on the surface of the neuron. The DA receptors are present on the post-synaptic neuron. $\boldsymbol{B}$. The activated neuron releases DA in synapse which binds to the DA receptors and activates it. C. DA then dissociates from the receptors and is either repackaged back to the neurons by DAT or is destroyed by the enzymatic degradation. The transportation of $D A$ is facilitated by $\mathrm{Na}^{+} / \mathrm{K}^{+}$ATPase. D. Cocaine competes with DA and binds to DAT leading to an increase in DA levels in the synapse. The freely available DA binds to the DA receptors and hyper-activates the post synaptic neuron. blue dots- DA; Orange dots- sodium ions; Red dotchloride ions; Dotted lines indicates decreased activity; Straight lines indicates increased activity; Red circle-storage vesicles for DA. 


\subsection{The kappa opioid receptors}

The KOPr is a member of 7 transmembrane G-protein coupled receptor protein (Eguchi, 2004) and the endogenous neuropeptide dynorphin (DYN; Goldstein et al., 1979) binds with high affinity to the KOPr (Corbett et al., 1982; Chavkin et al., 1982). High levels of KOPr's are found in the NAc, caudate putamen (CPU), claustrum, amygdala, endoperiform nucleus, whereas low levels of KOPr's were observed in the VTA and substantia nigra (Mansour et al., 1995; 1996; Quirion et al., 1987). Correspondingly, high levels of DYN were detected in the forebrain, hypothalamus and substantia nigra, whereas low levels of DYN have been reported in the dorsal and ventral striatum, amygdala and hippocampus (Goldstein and Ghazarossian, 1980; Webber et al., 1982). Although, pharmacological studies have indicated three subtypes of KOPr's, to date only one subclass of KOPr, KOPr1, has been cloned (Connor and Kitchen, 2006).

The mechanism by which the KOPr activates is shown in Fig 1.3. The inhibitory G-protein coupled receptors are associated with the intracellular G-protein heterodimer (Eguchi, 2004). The G-protein heterodimer comprises of $G_{\alpha}, G_{\beta}$ and $G_{\gamma}$ subunits (Connor et al., 1999). Upon activation of the KOPr, the G-protein trimer undergoes conformational changes followed by the dissociation of the $G_{\alpha}$ and $G_{\beta \gamma}$ subunits (Salamon et al., 2002). The dissociated subunits further modulate other signal transduction systems for example; the $G_{\alpha}$ subunit reduces adenylyl cyclise activity which decreases cyclic adenosine monophosphate (cAMP) induced protein kinase A signalling (Watts and Neve, 2005). The $\mathrm{G}_{\beta \gamma}$ subunit directly activates the inwardly rectifying potassium channel and inhibits voltage gated calcium channels (Holtz et al., 1998). Overall, this stabilizes the neuron by maintaining its resting state potential ( $\mathrm{Lu}$, 2004). The following section describes the activation of the KOPr/DYN negative feedback loop in response to repeated exposures to cocaine. 


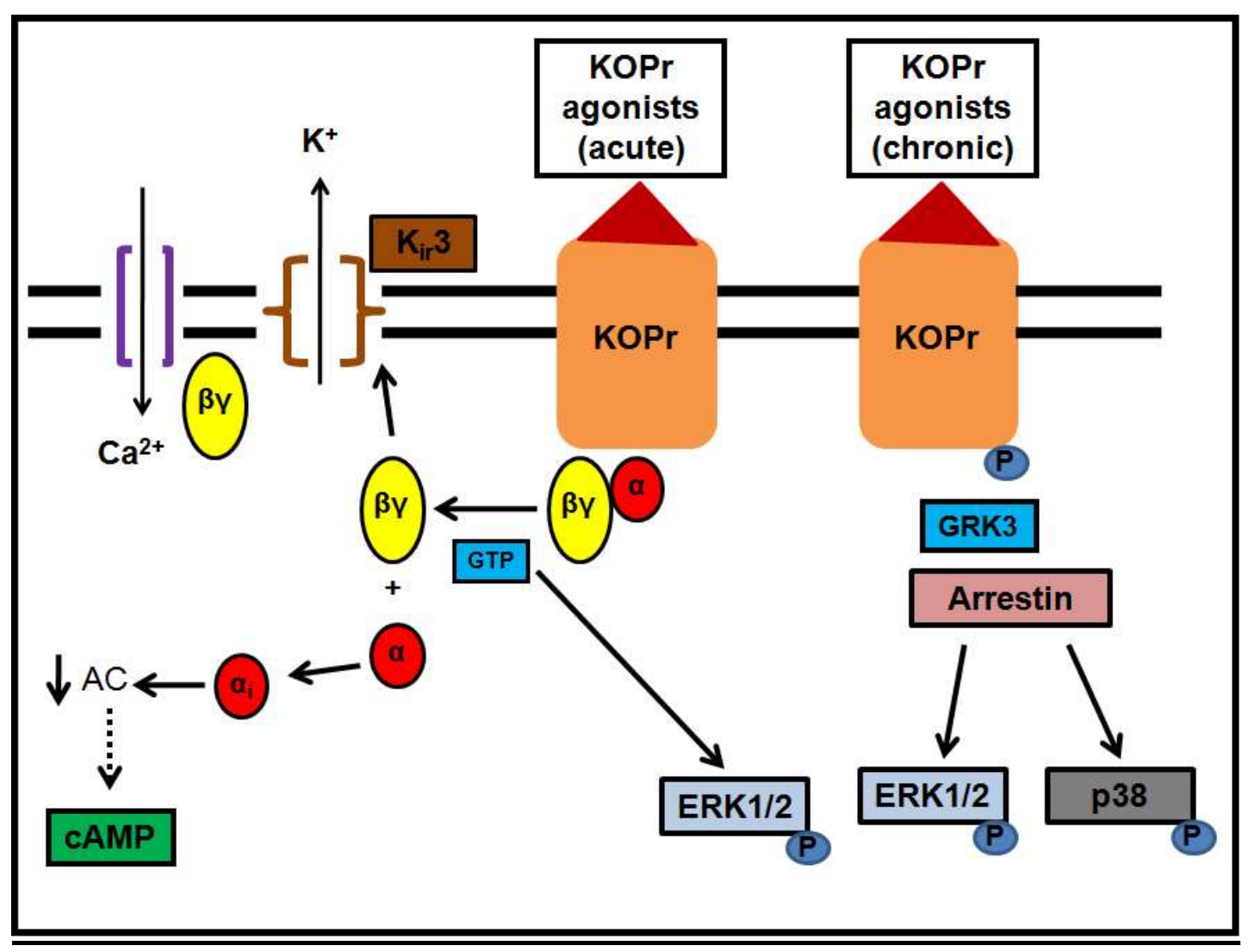

Fig.1.3. Signal transduction mechanism for kappa opioid receptor

\section{activation}

(Adapted from Bruchas and Chavkin, 2010). The signal transduction mechanism following acute and chronic KOPr activation. KOPr is an inhibitory GPCR which has $G_{\alpha \beta \gamma}$ subunits. On $K O P r$ activated, these subunits dissociates into $G_{\alpha}$ and $G_{\beta \gamma}$ subunits. The $G_{\alpha}$ subunit decreases the levels of adenylyl cyclase and the $G_{\beta \gamma}$ subunit modulates intracellular potassium and calcium ion levels. KOPr activation also leads to activation of protein kinases ERK1/2 and p38. However, long term activation of KOPr leads to receptor phosphorylation and kinase activation via GRK3-Arrestin dependent mechanism. KOPr- kappa opioid receptor; $K^{+}$- potassium ion; $K_{i r} 3-G$-protein gated inward rectifying $\mathrm{K}^{+}$channel; $\mathrm{Ca}^{2+}$ - calcium ion; $\alpha$ - G protein alpha subunit; $\beta \gamma$ - G protein beta-gamma subunit; GTP-Guanosine tri-phosphate; AC-adenylyl cyclise; cAMP- cyclic adenosine mono-phosphate; P- phosphorylation; GRK3- G-protein coupled receptor kinase 3; ERK1/2- extra-cellular signal-regulated kinase; p38 - p38 mitogen activated protein kinase. 


\subsubsection{Negative feedback loop and DYN/kappa opioid system}

The DYN/KOPr mediated negative feedback loop is presented in Fig 1.4. The gamma amino butyric acid - medium spiny neurons located in the NAc receives DAergic inputs from the VTA (Van Bockstaele and Pickel, 1995). This neuron expresses DA 1 receptors, which are coupled to stimulatory G-protiens (Carlezon et al., 1998) and functions by increasing the adenylyl cyclise activity. The increased cAMP activity enhances the transcription of CREB resulting in release of the endogeneous KOPr neuropeptide, DYN (Carlezon et al., 1998; Nestler, 2001). The spiny projections from NAc releases DYN at the pre-synaptic DA neurons where it binds to the presynaptic KOPr (Carlezon et al., 1998). The activated KOPr's has shown to oppose the hedonic effects produced by the drugs of abuse. (Nestler 2001; Shippenberg et al., 2007; Mysels and Sullivan, 2009). An increase in KOPr expression has been observed during chronic cocaine administration in NAc shell, CPU, claustrum and endopiriform nucleus (Collins et al., 2002). This is also accompanied by an elevation in striatal DYN levels and pro-DYN gene expression levels (Sivam, 1989; Daunais et al., 1995; Ramouldi et al., 1996). Agonists at KOPr's have shown to suppress elevation in DA levels in the midbrain dopaminergic systems (Di Chiara and Imparato 1988a; 1988b; Shippenberg et al., 1996). Therefore, activation of KOPr during the initial stages of the addiction cycle or prior to the drug withdrawal might help in curbing the development and progression of cocaine addiction (Shippenberg et al., 2007; Hasabe et al., 2004; Mysels, 2009; Prisinzano et al., 2005). This hypothesis has led to the idea of developing KOPr agonists as anti-addiction agents (Mysels and Sulliva, 2009; Shippenberg et al., 2007). The following section gives information on the endogeneous, synthetic and naturally derived KOPr agonists. 


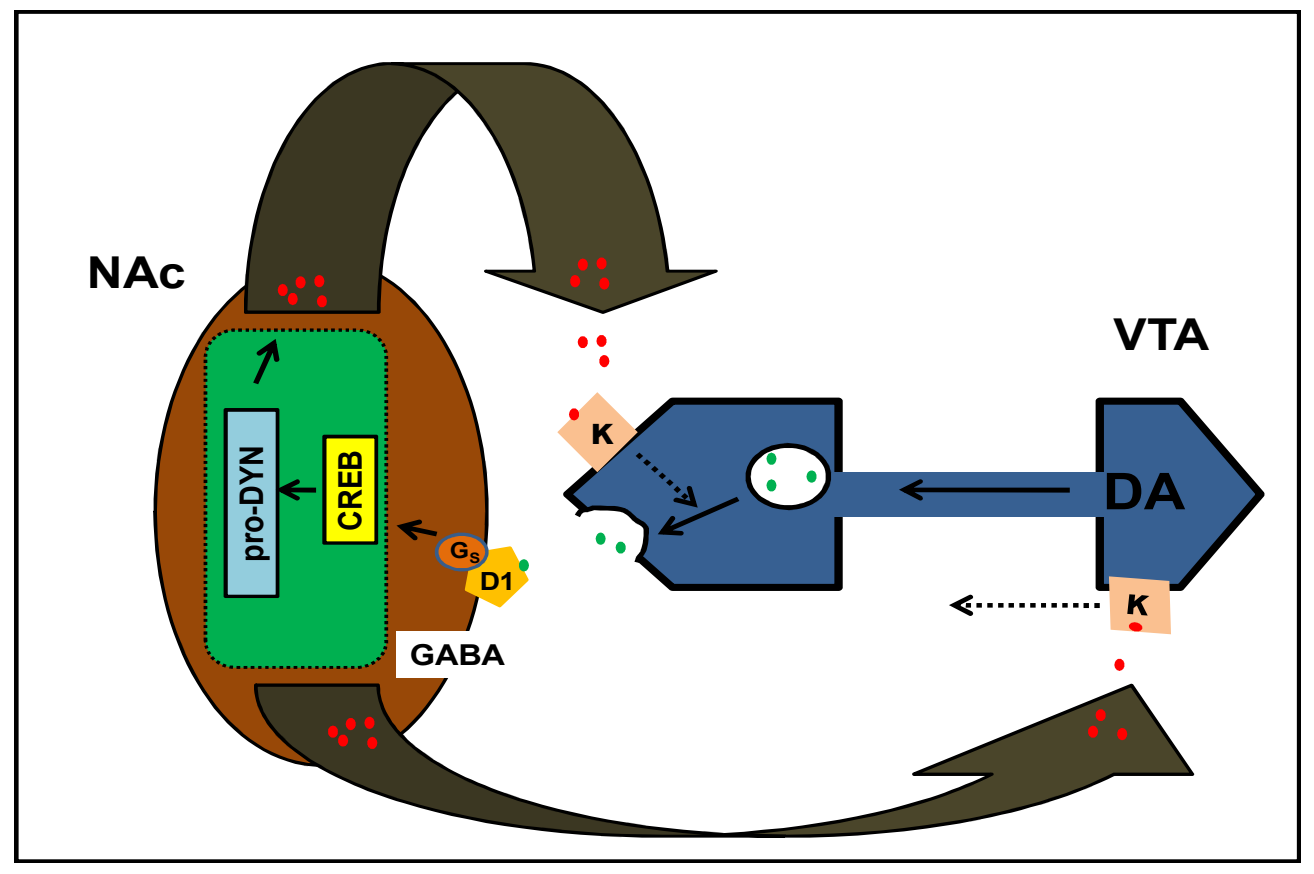

\section{Fig.1.4.Negative feedback loop}

(Adapted from Carlezon et al., 1998; Nestler, 2001). Opposing effects to the initial rewarding behaviours produced by drugs of abuse via the negative feedback loop. The NAc receives DA inputs from the VTA which activates the DA 1 receptor present on the gamma amino butyric acid medium spiny neurons. Upon activation, these neurons activate CREB, which releases the neuropeptide DYN at the presynaptic neurons. DYN binds to the KOPr and opposes the hedonic properties of cocaine via modulating DA tone in NAc. NAc-nucleus accumbens; GABA-gamma amino butyric medium spiny neuron; VTA-ventral tegmental area; CREB- cAMP response element binding protein; pro-DYN- prodynorphin gene; DA-dopaminergic neuron commencing from VTA; D1- DA 1 receptor; $G_{s^{-}}$Stimulatory G-protein coupled receptors; green dotsdopamine; k- kappa opioid receptor; red dots- DYN. 


\subsubsection{Kappa opioid receptor agonists}

The endogeneous and synthetic KOPr agonists are shown in Fig 1.5. The KOPr agonists consist of endogeneous peptide DYN (1-17) (Goldstein et al., 1980; Chavkin et al., 1982), arylacetamide analogues (U50488H; Vonvoigtlander et al., 1982; 1983, U69593; Lahti et al., 1985, spiradoline; Lahti et al., 1985); benzomorphans (bremazocine, Romer et al., 1980; cyclazocine, pentazocine, Harris and Pierson, 1964); epoxymorphinans (nalfurafine; Nagase et al., 1988); iboga alkaloids (ibogaine and its structural derivatives; Maisonneuve and Glick, 2003) and neoclerodane diterpenes derived from the plant Salvia divinorum (Sal A and its structural derivatives; Roth et al., 2002). The coming sections shall give an account of the behavioural anti-addiction as well as the adverse effect profile of KOPr agonists. 


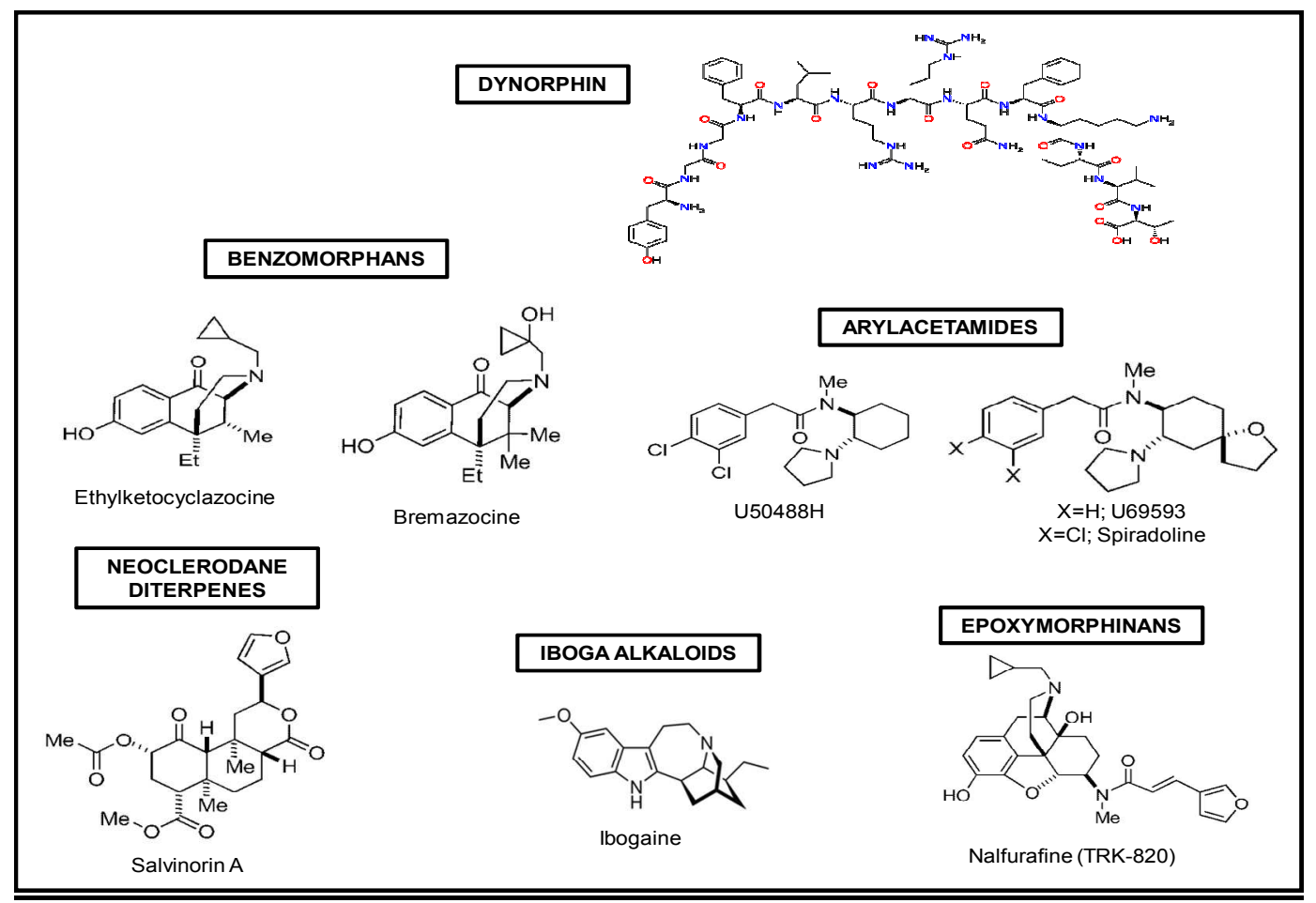

\section{Fig. 1.5. Kappa opioid receptor agonists}

(Modified from Eguchi, 2004; Baumann et al., 2001; Tachibana et al., 1982) Kappa opioid receptor agonists consist of the naturally occurring endogenous ligand DYN. Synthetic classes of agonists includes benzomorphans (ethylketazocine, bremazocine, cyclazocine); arylacetamides (U50488H, U69593, spiradoline); epoxymorphinans (nalfurafine); neoclerodane diterpenes (Sal A and its derivatives); Iboga alkaloids (ibogaine and its derivatives) 


\subsubsection{Kappa opioid agonists in pre-clinical models of addiction}

The effect of KOPr activation on cocaine produced behaviours has been studied extensively in laboratory animals and summarised in Tab 1.2.

\subsubsection{Arylacetamides}

Acute $\mathrm{KOPr}$ activation by the arylacetamide $\mathrm{KOPr}$ agonist $\mathrm{U} 50488 \mathrm{H}$ and U69593 attenuated nicotine induced behaviours in rats (Hahn et al., 2000; Ismailova and Shoaib, 2010). U69593 has been shown to attenuate self administration of heroin (Xi et al., 1998), and suppress amphetamine induced behaviours (Gray et al., 1999) in laboratory animals. U50488H and spiradoline both dose-dependently attenuated selfadministration of cocaine and morphine in rats, which was reversed by KOPr antagonist pre-treatment (Glick et al., 1995; Kuzmin et al., 1997). The same study also showed that at a higher dose, both arylaceamides $(5,10 \mathrm{mg} / \mathrm{kg})$ decreased water consumption in rats (Glick et al., 1995). Thus, suggesting a non-selective attenuation at higher dose. $\mathrm{U} 50488 \mathrm{H}$ has also been shown to attenuate ethanol self-administration in rats (Lorgip et al., 2008). In a drug discrimination model, U50488H either had no effect (Broadbent et al., 2002) or attenuated rewarding effects produced by a low training dose of cocaine ( 3 $\mathrm{mg} / \mathrm{kg}$ vs. $10 \mathrm{mg} / \mathrm{kg}$; Kantak et al., 1999). U50488H, U69593 and spiradoline pretreatment attenuated cocaine-induced hyperactivity (Vanderschuren et al., 2000) and behavioural sensitization (Heidbreder et al., 1996; Puig-Ramos et al., 2008) in rats. Additionally, spiradoline attenuated morphine induced behavioural sensitization (Smith et al., 2003) and cross sensitization to cocaine (Smith et al., 2009). Also, U50488H, U69593, and spiradoline all attenuate cocaine prime induced reinstatement in rats (See et al., 2010; Morani et al., 2009; Schenk et al., 2000). These findings indicate that arylacetamide KOPr agonists produce anti-addiction like effects in laboratory animals which might be non-selective (Glick et al., 1996). 


\subsubsection{Benzomorphans}

The benzomorphan analogue, cyclazocine has also been shown to attenuate cocaine self-administration without affecting water consumption in rats (Glick et al., 1998). However, in rhesus monkeys, bremazocine but not cyclazocine attenuated cocaine maintained behaviours (Negus and Mello, 2000). Bremazocine also attenuated self-administration of cocaine and oral ethanol with decreased saccharin consumption in rhesus monkeys (Cosgrove and Carrol, 2002). However, in another study, bremazocine induced attenuation of freely available ethanol was without any effect on sucrose consumption in rats (Nestby et al., 1999). Also, bremazocine pre-treatment attenuated cocaine and amphetamine induced enhancement in motor function, and amphetamine behavioural sensitization in laboratory animals (Vanderschuren et al., 2000). Collectively, these findings imply the role of benzomorphan compounds in producing anti-addiction effects.

\subsubsection{Epoxymorphinans}

The epoxymorphinan analogue, nalfurafine has also been shown to attenuate cocaine discriminative stimulus effects in a KOPr specific manner (Mori et al., 2002). Furthermore, nalfurafine attenuated rewarding and locomotor effects of cocaine in mice (Hesebe et al., 2004). Also, low doses of nalfurafine $(10,20 \mu \mathrm{g} / \mathrm{kg})$ did not produce place preference or aversion (Mori et al., 2002; Hesebe et al., 2004). However, at high dose $(80 \mu \mathrm{g} / \mathrm{kg})$ it induces conditioned place aversion in laboratory animals. Thus suggesting that place aversion produced by nalfurafine is dose related.

\subsubsection{Phytochemical KOPr agonists}

Naturally derived KOPr agonists such as iboga alkaloids and neoclerodane diterpenes have been tested for their anti-addiction effects using animal models. Pre- 
treatment with ibogaine, an alkaloid derived from plant Tabermanthe iboga (Family: Apocynaceae) led to decreased cocaine induced hyperactivity and cocaine selfadministration in laboratory animals (Cappendijk and Dzoljic, 1993; Sershen et al., 1992). Synthetic derivatives of ibogaine have also been shown to be effective in suppressing cocaine produced behaviours (Maissaneuve and Glick, 2003). In addition to its cocaine antagonist actions, ibogaine also attenuates DA sensitization to cocaine treatment (Szumlnski et al., 2000), thus explaining its anti-addiction effects.

Taken together, these results suggests that KOPr activation by arylacetamides, benzomorphans, epoxymorphinans and phytochemicals such as ibogaine and Sal A produce anti-addiction behaviours in laboratory animals. Because of the behavioural pharmacology of KOPr agonists, they have been proposed for development as antiaddiction pharmacotherapeutics (Neumeyer et al., 2000; Mello and Negus, 2000; Shippenberg et al., 2001; Prisinzano et al., 2005; Shippenberg et al., 2007). KOPr agonists oppose the DA sensitizing effects produced by cocaine and this has been suggested as a possible mechanism by which KOPr activation produces anti-addiction effects. The following section briefly describes the mechanism of the anti-addiction effects produced by KOPr activation. 


\subsubsection{Mechanism of KOPr agonist induced anti-addiction effects}

KOPr agonist pre-treatment has been shown to attenuate extracellular DA levels in the reward circuits (Shippenberg et al., 1996). Direct infusion of DYN in the NAc has been shown to decrease DA levels in the MDS (Zhang et al., 2004). Additionally, intracerebroventricular (icv) or systemic injections of U50488H and U69593 decreases DA levels in NAc (Spanagel et al., 1992; Devine et al., 1993; Di Chiara and Imparato, 1988a; 1988b; Maisonneuve et al., 1994; Shippenberg et al., 1996; Heidbreder et al., 1996; Thompson et al., 2000). This effect was reversed by nor-BNI pre-treatment (Spanagel et al., 1990; 1992). Furthermore, when the VTA was directly pre-treated with KOPr agonists a decrease in DA levels in the PFC was seen (Margolis et al., 2003; 2006). Also, pre-treatment with another arylacetamide analogue R-84760 and the novel KOPr agonist Sal A has been shown to dose dependently attenuate DA levels in CPU (Zhang et al., 2004; Zhang et al., 2005; Gherke et al., 2008). Systemic treatment with Sal A has also been shown to decrease DA levels in the NAc (Carlezon et al., 2006), and cocaine produced cFOS expression in the CPU was also reduced (Chartoff et al., 2008). KOPr activation by U69593 has also been shown to decrease cocaine induced dopamine- and adenosine $3^{\prime}, 5^{\prime}$-monophosphate-regulated protein with phosphorylation, which seems to play an important role in cocaine produced locomotor sensitization (D'Addario et al., 2007). Collectively, these findings show that KOPr activation opposes cocaine induced behaviours by decreasing extracellular DA levels in the NAc, thus indirectly acting as cocaine antagonists (Shippenberg et al., 2001; Prisinzano et al., 2005). The adverse effects however, associated with KOPr activation (depression, aversion, sedation) have limited their further clinical development. The following section describes the behavioural adverse effects associated with KOPr activation and the mechanisms that may mediate these behaviours. 


\section{Tab1.2. KOPr agonists in pre-clinical studies of addiction}

\begin{tabular}{|c|c|c|c|}
\hline Pharmacological effect & KOPr agonists tested & Behaviour produced & References \\
\hline \multirow[t]{4}{*}{ Cocaine self-administration } & $\mathrm{U} 50488 \mathrm{H}$ & Attenuate & $\begin{array}{l}\text { Glick et al., 1995; Kuzmin } \\
\text { et al., 1997; Negus et al., } \\
1997\end{array}$ \\
\hline & Spiradoline & Attenuate & $\begin{array}{l}\text { Wadenberg, 2003, Glick et } \\
\text { al., } 1995\end{array}$ \\
\hline & Bremazocine & Attenuate & Cosgrove and Carrol, 2002; \\
\hline & Iboga alkaloids & Attenuate & $\begin{array}{l}\text { Coppendijk and Dzoljic, } \\
1993\end{array}$ \\
\hline Cocaine motor sensitization & U50488H, U69593 & Attenuate & $\begin{array}{l}\text { Heidbreder et al., 1993; } \\
\text { 1995; Collins et al., 2001a; } \\
\text { 2001b; Puig-Ramos et al., } \\
2008\end{array}$ \\
\hline $\begin{array}{l}\text { Cocaine induced brain } \\
\text { stimulation reward }\end{array}$ & U69593 & Attenuate & Tomasaiwicz et al., 2008 \\
\hline \multirow{3}{*}{$\begin{array}{l}\text { Cocaine induced discriminative } \\
\text { stimulus effects }\end{array}$} & $\mathrm{U} 50488 \mathrm{H}$ & No effect & Broadbent et al., 2002 \\
\hline & U50488H, Cl-977 & $\begin{array}{l}\text { Attenuates at low training dose of } \\
\text { cocaine }(3 \mathrm{mg} / \mathrm{kg})\end{array}$ & $\begin{array}{l}\text { Kantak et al., 1999; } \\
\text { Spealman and Bergman; } \\
1992\end{array}$ \\
\hline & Nalfurafine & Attenuate & Mori et al., 2002 \\
\hline $\begin{array}{l}\text { Cocaine induced reinstatement } \\
\text { (self-administering rats) }\end{array}$ & $\begin{array}{l}\text { U50488H, U69593, } \\
\text { spiradoline, } \\
\text { Sal A }\end{array}$ & Attenuate & $\begin{array}{l}\text { Schenk et al., 1999b; } \\
\text { 2000a; Morani et al., } 2009\end{array}$ \\
\hline $\begin{array}{l}\text { Cocaine induced enhancement } \\
\text { in CPP }\end{array}$ & U69593, U50488H & Attenuate & Shippenberg et al., 1996 \\
\hline \multirow[t]{6}{*}{ Cocaine induced hyperactivity } & U69593 & Attenuate & $\begin{array}{l}\text { Collins et al., 2001a, } \\
\text { 2001b; Vanderschuren et } \\
\text { al., } 2000\end{array}$ \\
\hline & U50488H & Attenuate & $\begin{array}{l}\text { Vanderschuren et al., 2000; } \\
\text { Heidbreder et al., } 1995\end{array}$ \\
\hline & Nalfurafine & Attenuate & Hasabe et al., 2004 \\
\hline & Sal A & Attenuate & Chartoff et al., 2008 \\
\hline & $\mathrm{R}-84760$ & Attenuate & Zhang et al., 2004 \\
\hline & Bremazocine & Attenuates & Vanderschuren et al., 2000 \\
\hline \multirow[t]{5}{*}{$\begin{array}{l}\text { Effect on midbrain dopamine } \\
\text { release }\end{array}$} & $\mathrm{U} 50488 \mathrm{H}$, & Decreases in NAc & $\begin{array}{l}\text { Di Chiara and Imparato, } \\
\text { 1988a,1988b; Maisonneuve } \\
\text { et al., 1994; }\end{array}$ \\
\hline & U69593 & Decreases in NAc & $\begin{array}{l}\text { Shippenberg et al., 1996; } \\
\text { Thompson et al., } 2000\end{array}$ \\
\hline & $\mathrm{R}-84760$ & Decreases in CPU & Zhang et al., 2004 \\
\hline & Sal A & Decrease in NAc and CPU & $\begin{array}{l}\text { Carlezon et al., 2006; } \\
\text { Gherke et al., 2008, Zhang } \\
\text { et al., 2005. }\end{array}$ \\
\hline & Ibogaine & Decrease DA sensitization & Szumlinski et al., 2000 \\
\hline $\begin{array}{l}\text { Amphetamine induced } \\
\text { behaviours }\end{array}$ & U69593 & Attenuate & Gray et al., 1999 \\
\hline Morphine self-administration & $\mathrm{U} 50488 \mathrm{H}$ & Attenuate & $\begin{array}{l}\text { Glick et al., 1995; Kuzmin } \\
\text { et al., } 1997\end{array}$ \\
\hline $\begin{array}{l}\text { Morphine induced rewarding } \\
\text { behaviours }\end{array}$ & Spiradoline & Attenuate & $\begin{array}{l}\text { Glick et al., 1995; } \\
\text { Smith et al.,2003; } 2009\end{array}$ \\
\hline Heroin self-administration & $\mathrm{U} 50488 \mathrm{H}$ & Attenuates & Xi et al., 1998 \\
\hline Nicotine self-administration & $\begin{array}{l}\text { U69593, U50488H, CI- } \\
977\end{array}$ & Attenuate & $\begin{array}{l}\text { Hahn et al., 2000; } \\
\text { Ismailova and Shoaib, } \\
2010\end{array}$ \\
\hline \multirow[t]{2}{*}{ Ethanol self-administration } & Bremazocine & Attenuate & Nestby et al., 1999 \\
\hline & U50488H & Attenuate & Lorgip et al., 2008 \\
\hline
\end{tabular}




\subsubsection{Behavioural adverse effects of KOPr agonists}

\subsubsection{Sedation and psychotomimic effects}

KOPr agonists U50488H, bremazocine, spiradoline and U69593 have been shown to decrease spontaneous open field activity in laboratory animals (Von Voigtlander et al., 1983; Lahti et al., 1982; 1985; Wadenberg, 2003). Furthermore, KOPr activation by Sal A and U69593 induces hallucinations in non-human primates (Butelman et al., 2007; 2009; 2010). Clinical studies showed that acute administration of enadoline, a selective $\mathrm{KOPr}$ agonist produces sedation, emesis, hallucinations, dizziness, excessive urination and psychotomimesis in humans (Walsh et al., 2001a). Moreover, KOPr activation by the benzomorphan analogue, Mr2033 also produces psychotomimetic actions in humans (Pfeiffer et al., 1996). These adverse behaviours produced by KOPr agonists have discouraged the development of KOPr agonists as potential anti-addiction agents (Wee and Koob, 2010).

\subsubsection{Depression and aversion.}

The KOPr agonists have been shown to produce depressive behaviours in laboratory animals. Both U69593 and Sal A dose dependently increase immobility time in the forced swim test (FST; Mague et al., 2003; Carlezon et al., 2006). Additionally, pre-treatment with U50488H, U69593 and Sal A increases the ICSS threshold in rats (Todtenkopff et al., 2004; Ebner et al., 2010; Dinieri et al., 2009). These data indicate that KOPr activation produces depressive effects in laboratory animals. In addition to its depressive effects, KOPr activation also produces aversive behaviours as pre-treatment with $\mathrm{U} 50488 \mathrm{H}$, bremazocine, ethylketocyclazocine or U69593 has been shown to induce conditioned -place and -taste aversion in laboratory animals (Shippenberg and Herz, 1986; Mucha and Herz, 1985). The following section gives an account of the mechanisms underlying KOPr activation mediated depressive and aversive behaviours. 


\subsubsection{Nucleus accumbens and KOPr agonists induced depression and aversion.}

Direct or systemic injection of U69593 into the NAc has been shown to induce aversive behaviours in laboratory animals, which were reversed by pre-treatment with the selective KOPr antagonist, nor-binaltorphimine (nor-BNI; Bals Kubik et al., 1989; 1993). The aversion induced by KOPr activation is believed to involve DA neurotransmission in the NAc, as direct application of the DYN peptide E2078 to the NAc reduces release of phasic DA. This effect was reversed by KOPr antagonist pretreatment (Spanagel et al., 1990).

Another reason for the depressive effects produced by KOPr agonists might be due to the phosphorylation of CREB in the NAc (Carlezon et al., 1998; Nestler et al., 2001; Hyman and Malenka, 2001; Carlezon et al., 2005; Dinieri et al., 2009). Recent results suggests that enhancement in levels of phosphorylated CREB in NAc is associated with increased immobility time in the forced swim test (FST; Plaikas et al., 2001). KOPr agonists have shown to increase time spent immobile in FST and also increase CREB levels in NAc (Mague et al., 2003; Carlezon et al., 2006). On the other hand blocking central KOPr's opposes depressive effects in FST and also reduces CREB phosphorylation in the NAc (Mague et al., 2003; Chartoff et al., 2009).

Collectively, this data strongly suggests that NAc is one of the major sites for KOPr activation induced depression (Nestler et al., 2001; Carlezon et al., 2009) and alterations in DA neurotransmission and CREB in NAc plays a major role in the pathophysiology of KOPr mediated depressive behaviours (Knoll and Carlezon, 2010; Carlezon et al., 2009). 


\subsubsection{Central 5HT systems and KOPr activation induced depression and aversion.}

KOPr have been located in brain regions such as raphae nucleus, NAc, hippocampus which are rich in 5HT neurotransmission (Battaglia et al., 1991; Pinnock, 1992). Depletion of 5HT by parachlorophenylalanine (a synthetic amino acid which depletes serotonin from cell body) attenuated the analgesic effects of U50488H (Nemmani and Mogil, 2003). Whereas prior treatment with selective serotonin reuptake inhibitor; fluoxetine (SSRI; FLX) potentiated U50488H induced analgesic effects in mice (Nemmani et al., 2001). Additionally, 5HT is also believed to play a key role in controlling DYN levels. The depletion of 5HT has also been shown to decrease proDYN mRNA expression by approximately $60 \%$ in CPU, hippocampus and hypothalamus (Di Addario et al., 2007). These findings suggest the importance of 5HT systems in KOPr agonist induced behaviours. Moreover, modulation of 5HT systems by KOPr agonists have been implicated in cocaine induced behaviours. A recent study by Zakharova et al., (2008) suggested that depletion of 5HT from the brain decreased the ability of U69593 to attenuate cocaine induced motor stimulation. Additionally, selective SERT inhibitors FLX $(5.6 \mathrm{mg} / \mathrm{kg})$ and citalopram $(10 \mathrm{mg} / \mathrm{kg})$ attenuated cocaine induced drug-seeking behaviour in squirrel monkeys (Ruedi-Bettschen et al., 2010). Moreover, the same study showed that this effect produced by KOPr agonist enadoline and FLX was reversed by the pre-treatment with 5HT 2A receptor agonist 8OH-DPAT (Ruedi-Bettschen et al., 2010). Thus, KOPr activation may modulate 5HT systems which, in turn also modulate cocaine induced behaviours (Ruedi-Bettschen et al., 2010).

The 5HT systems might be implicated in the KOPr agonist's induced adverse effects as KOPr activation has been shown to modulate the 5HT neurotransmission in various brain regions. Yilmaz and colleagues, (2006) reported that icv administration of U50488H significantly decreased the tissue levels of 5HT and its metabolite, 5- 
hydroxyindole acetic acid in hippocampus and hypothalamus. These effects were prevented by nor-BNI pre-treatment. Also, a significant reduction (approx 30\%) in 5HT concentrations using in vivo microdialysis following local infusions of U50488H (300 $\mu \mathrm{M}$ ) was observed in the dorsal raphae nucleus (Tao and Auerbach, 2005). A, recently published report by Land et al., (2009) implicate 5HT projections from the dorsal raphae nucleus to NAc in KOPr activation and repeated stress induced aversion. These results strongly suggest that central KOPr activation alters 5HT neurotransmission. This may account for the KOPr mediated adverse effects and manipulation of 5HT systems might help in alleviating these adverse effects. 


\subsubsection{Effect of acute vs. chronic KOPr activation.}

Previous studies have shown that intravenous (iv) infusions $(23 \mathrm{hr} /$ day for 10 consecutive days) of the KOPr agonist bremazocine $(0.0032 \mathrm{mg} / \mathrm{kg} / \mathrm{hr})$; enadoline (0.001, $0.0032 \mathrm{mg} / \mathrm{kg} / \mathrm{hr})$; ethylketazocine (0.01, $0.0032 \mathrm{mg} / \mathrm{kg} / \mathrm{hr}) ; \operatorname{Mr} 2033(0.01$, $0.0032 \mathrm{mg} / \mathrm{kg} / \mathrm{hr})$ and $\mathrm{U} 50488 \mathrm{H}(0.1 \mathrm{mg} / \mathrm{kg} / \mathrm{hr})$ decreased cocaine self-administration in rhesus monkeys (Negus et al., 1997; Mello and Negus, 1998; 2000). This attenuation was also accompanied by a significant reduction in food reinforcement, sedation and emesis, which subsided after 2-3 days of treatment (Mello and Negus, 2000). Acute exposure to these compounds also suppressed food reinforcement in rhesus monkeys (Mello and Negus, 2000). The enadoline and Mr2033 induced reduction in cocaine selfadministration reverted to baseline levels of cocaine self-administration during last week of treatment, when these compounds were administered for 28 subsequent days (Mello and Negus, 2000). This implies that chronic KOPr activation had no effect on cocaine's reinforcing effects in rhesus monkeys. Another study by Negus, (2004), however showed that monkeys trained to discriminate between food and cocaine using concurrent choice paradigm, when continuously infused with U50488H $(0.032 \mathrm{mg} / \mathrm{kg} / \mathrm{hr}$, iv infusion) showed cocaine preference. The same study also showd that this effect was KOPr dependent as nor-BNI pre-treatment reversed it (Negus, 2004). This result suggests that continuous KOPr activation might enhance cocaine's reinforcing properties in comparison to natural reward such as food.

On the other hand, a study by Preston and colleagues (2004) showed that acute oral administration of cyclazocine $(0.2-0.8 \mathrm{mg})$ for 4 consecutive days significantly decreased the acute subjective effects of cocaine in humans. Moreover, acute administration of $\mathrm{KOPr}$ agonists attenuated cocaine self-administration and reinstatement in rats (Glick et al., 1995; Kuzmin et al., 1997; Schenk et al., 1999; 2000; Morani et al., 2009). These findings show differences in acute and chronic KOPr 
treatment on cocaine induced behaviour and support the short term use of KOPr agonists in attenuating the effects of cocaine.

The effects produced by KOPr agonists on DA levels in the NAc may depend upon the frequency of KOPr activation, as acute administration of U69593 inhibits the potassium ion evoked DA release from rat striatal synaptosomes which was reversed by nor-BNI pre-treatment (Ronken et al., 1993). In contrast, repeated administration of U69593 significantly increased potassium ion evoked DA levels in the NAc (Fuentelaba et al., 2006) and in cultured mesencephalonic DA neurons (Ronken et al., 1993). Moreover, acute administration of U69593 directly into the NAc of mice has been shown to attenuate DA release (Chefer et al., 2005). Whereas, repeated administration of U69593 decreases DA uptake without affecting extracellular DA levels in the NAc (Thompson et al., 2000). These data further strengthen the role of acute KOPr activation in producing desired anti-addiction effects at cell and tissue levels.

Chronic KOPr activation leads to receptor deactivation via KOPr phosphorylation and may cause KOPr internalization as decreased KOPr binding was observed after chronic treatment with $\mathrm{U} 50488 \mathrm{H}$ in vitro (Bhargava et al., 1989). Also, chronic KOPr treatment activate protein kinase (p38 mitogen activated protein kinase and extracellular signal-regulated kinases 1 and 2; Bruchas et al., 2006; Belcheva et al., 2005). However, differences in acute and chronic KOPr activation mediated phosphorylation of protein kinases have been reported (McLennan et al., 2009; Fig 1.3). The KOPr mediated signal transduction mechanism has been extensively reviewed by Carr and Mague, (2008); Bruchas and Chavkin, (2010) and is shown in Fig 1.3. Taken together, these findings suggest that acute but not chronic KOPr activation might be beneficial in producing anti-addiction effects.

A study by Walsh and colleagues, (2001b) showed that, acute administration of selective KOPr agonist enadoline attenuated subjective effects of cocaine without 
having any effect on cocaine self-administration in humans. Moreover, enadoline produced compliance problems and psychotomimesis when tested in healthy subjects (Walsh et al., 2001a). Thus, despite having anti-addiction properties, these adverse effects have limited the development of KOPr agonists as anti-addiction pharmacotherpies. Recently, Sal A, was reported to be a novel, naturally occurring, potent and selective KOPr agonist. One of the aims of this thesis was to determine behavioural anti-addiction and side effect profile of Sal A. The following section gives a detailed account on the behavioural pharmacology of Sal A. 


\subsection{Salvinorin $A$}

Sal A is the selective KOPr agonist found in the plant Salvia divinorum (family:Lamiaceae; Roth et al., 2002). This plant has been used for spiritual and divination purposes by the Mazatecs from Oaxaca, Mexico for centuries (Ott, 1995; Siebert, 1994; Valdez et al., 1984; Valdez, 94). Smoking dried Salvia divinorum leaves is associated with intense hallucinations of short duration (Valdez, 1994; Siebert, 1994). Sal A has a novel neoclerodane diterpene structure and has been shown to bind to the inhibitory $\mathrm{G}_{\alpha \mathrm{i}}$ protein coupled KOPr (Roth et al., 2002). Sal A is selective in binding and activating KOPr expressing in the human embryonic kidney (HEK -293) cells and at native KOPr expressed in guinea pig brain (Roth et al., 2002). Furthermore, Sal A has no affinity for MOPr or DOPr (Roth et al., 2002; Yan and Roth 2004; Chavkin et al., 2004: Vorthermes and Roth 2006). Also, Sal A showed no affinity for DA, serotonin (5HT), muscarinic acetylcholine, adrenergic, cannabinoid or sigma receptors, which was measured using functional binding assays (Roth et al., 2002). Thus, Sal A is a unique hallucinogen without any affinity for 5HT $2 \mathrm{~A}$ receptors, which are the primary target for classic hallucinogens (Roth et al., 2002; Vorthermes and Roth, 2006). Taken together, this data suggests that hallucinations produced by Sal A are via KOPr activation (Roth et al., 2002; Killinger et al., 2010).

In vitro binding studies have shown that Sal A binds with greater affinity to KOPr than the arylacetamide KOPr agonists U50588H and U69593, and with equal affinity to the endogeneous KOPr ligand DYN (Chavkin et al., 2004). The incubation of Chinese hamster ovary cells stably expressing human KOPr with Sal A for $4 \mathrm{hr}$ induced a receptor down-regulation which was similar to other KOPr agonists tested (Wang et al., 2005). However, another study by Wang and colleagues (2008) showed that Sal A pre-treatment had no effect, whereas $\mathrm{U} 50488 \mathrm{H}$ pre-treatment induced 40 times more KOPr internalization in vitro. As Sal A has a different structure compared to 
arylacetamides (Fig 1.5), these observed differences might, at least be related to the structural dissimilarities noted between these two class of KOPr agonists.

\subsubsection{Behavioural pharmacology of Sal A}

The behavioural pharmacology of Sal A is summarised in Tab 1.3.

\subsubsection{Sedation, hallucinations and motor suppression.}

Sal A has shown to produce hallucinations in humans (Siebert et al., 1994; Valdes et al., 1983; Johnson et al., 2010) and induce sedative effects in non-human primates (Butelman et al., 2009; 2010). At doses of 1 and $3.2 \mathrm{mg} / \mathrm{kg}$ it produces a KOPr mediated, nor-BNI reversible decrease in locomotor activity in mice (Zhang et al., 2005). Furthermore, Sal A decreases climbing behaviour in mice in an inverted screen model (Fantagrossi et al., 2005). However, another study indicated that Sal A pretreatment $(1 \mathrm{mg} / \mathrm{kg}, 0.25-2 \mathrm{mg} / \mathrm{kg})$ had no effect on locomotor activity in rats (Hooker et al., 2008; Carlezon et al., 2006). Additionally, 2-methoxy-methyl salvinorin B (MOM Sal B), a more potent and longer acting Sal A analogue, produced a rapid, long lasting (about $3 \mathrm{hr}$ ) immobility in mice which was dose dependent and nor-BNI reversible (Wang et al., 2008). However, the same study showed that MOM Sal B increased locomotion in the Y-maze test in rats. Thus, indicating an effect which might be species specific.

\subsubsection{Antinociception}

Sal A has been shown to produce rapid anti-nociception (commencing within $10 \mathrm{~min}$ ) with short duration of effects (30 min) in laboratory animals (McCurdy et al., 2006; John et al., 2006). This effect produced by Sal A was KOPr mediated, as nor-BNI pre-treatment completely reversed it (McCurdy et al., 2006; Ansonoff et al., 2006). 
Furthermore, 2-propionate Sal A and MOM Sal B have been shown to produce antinociception in laboratory animals (Ansonoff et al., 2006; Wang et al., 2008).

\subsubsection{Drug Discrimination}

In drug discrimination studies, Sal A pre-treatment completely substituted for U69593 in rats paired with U69593 and conditioned to obtain food reinforcements (Willmore-Fordham et al., 2007). This effect was reversed by nor-BNI pre-treatment (Willmore-Fordham et al., 2007). Furthermore, the Sal A induced discriminative stimulus effects of U69593 in rats were also observed in non-human primates (Butelman et al., 2004). A recent study by Baker and colleagues, (2009) showed that the longer acting, more potent, Sal A analogues, 2-ethoxymethyl salvinorin B (EOM Sal B) and MOM Sal B also substituted for U69593 at doses lower than Sal A. These findings indicates a similar pharmacological effects induced by U69593, Sal A and its analogues in activating central KOPr's.

\subsubsection{Anti-cocaine effects}

Acute intraperitoneal (ip) administration of Sal A (2 mg/kg) has been shown to decrease cocaine induced hyperactivity in rats (Chartoff et al., 2008). This effect was dependent on context learning as Sal A, when injected in the activity boxes potentiated locomotor activity; whereas, administration of Sal A in the home cages did not potentiate motor function induced by the cocaine challenge (Chartoff et al., 2008). Furthermore, acute administration of Sal A, dose dependently $(0.3,1.0 \mathrm{mg} / \mathrm{kg})$ attenuated cocaine prime induced reinstatement of cocaine seeking without suppressing sucrose reinforcements or causing sedation in rats (Morani et al., 2009). These findings indicate that anti-cocaine behaviours are produced by Sal A, just like traditional KOPr 
agonists in laboratory animals. More work is necessary to characterize the therapeutic anti-addiction potential of Sal A-like compounds.

\subsubsection{Depression and Aversion}

Recent studies have shown that Sal A (2 mg/kg) dose dependently increases ICSS thresholds, decreases swimming time and increases immobility time in the FST paradigm in rats (0.25- $2 \mathrm{mg} / \mathrm{kg}$; Carlezon et al., 2006). Furthermore, Sal A (2 mg/kg) has been shown to suppress sucrose reinforcement in laboratory animals (Ebner et al., 2010). Sal A (1.0, $3.2 \mathrm{mg} / \mathrm{kg})$ pre-treatment has also been shown to induce conditioned place aversion in mice (Zhang et al., 2005). Additionally, the N-methylacetamide derivative of Sal A has also shown to produce long lasting increases in ICSS thresholds in rats (Beguin et al., 2008). However, in zebrafish, Sal A at high doses $(80 \mu \mathrm{g} / \mathrm{kg})$ induces place aversion, whereas at lower doses $(1 \mu \mathrm{g} / \mathrm{kg})$ induces place preference (Braida et al., 2007). Recent reports by Braida et al., (2009) suggests that Sal A pretreatment produces anti-depressant and anxiolytic effects using FST, tail suspension and elevated plus maze tests in laboratory animals. Also, lower dose of Sal A $(0.25,0.3$ $\mathrm{mg} / \mathrm{kg}$ ) did not suppress sucrose reinforcements in rats (Ebnar et al., 2010; Morani et al., 2010). Thus the aversive effects induced by Sal A are likely to be dose related.

\subsubsection{Toxicity studies}

Pre-clinical toxicological studies with Sal A show that Sal A does not produce any toxicity in mice, even at high dose (1000 mg/kg) (Mowry et al., 2003). These findings also show that chronic treatment (once every 14 days) of Sal A (dose range 0.4 $-6.4 \mathrm{mg} / \mathrm{kg}$ ) did not significantly alter the histology of liver, spleen, kidney, brain and bone marrow, or change cardiac function, heart rate or pulse pressure in laboratory animals (Mowry et al., 2003). Recent studies have also shown that Sal A administration 
in healthy volunteers did not produce any cardiovascular adverse effects, however, hallucinations were observed (Johnson et al., 2010; Siebert, 1994). Also, some incidences of Sal A induced psychosis have been reported clinically (Singh, 2007; Przekop and Lee, 2009; Paulzen and Grunder, 2008). Therefore, further detailed studies are warranted to establish the psychological safety profile for Sal A at pharmacological doses. 
Tab.1.3. Salvinorin A: Pre-clinical studies

\begin{tabular}{|l|l|}
\hline Pharmacological effect & Reference \\
\hline Anti-nociception & $\begin{array}{l}\text { John et al., 2006; McCurdy et al., 2006; } \\
\text { Ansenoff et al., 2006. }\end{array}$ \\
\hline Hallucination & Butelman et al., 2007; 2009; 2010. \\
\hline Sedation & $\begin{array}{l}\text { Zhang et al., 2005; Fantagrossi et al., 2005; } \\
\text { Butelman et al., 2009; 2010. }\end{array}$ \\
\hline Discriminative stimulus & $\begin{array}{l}\text { Willmore-Fordham et al., 2007; Butelman et al., } \\
\text { 2004; Baker et al., 2009. }\end{array}$ \\
\hline Depression & Carlezon et al., 2006; Ebnar et al., 2010. \\
\hline Conditioned place aversion & Zhang et al., 2005. \\
\hline $\begin{array}{l}\text { Anti-depression/ Anti- } \\
\text { anxiety }\end{array}$ & Braida et al., 2009; Braida et al., 2008. \\
\hline $\begin{array}{l}\text { Attenuate cocaine induced } \\
\text { behaviours }\end{array}$ & Chartoff et al., 2008; Morani et al., 2009. \\
\hline $\begin{array}{l}\text { Decreased dopamine levels } \\
\text { in MDS }\end{array}$ & $\begin{array}{l}\text { Carlezon et al., 2006; Gherke et al., 2008, } \\
\text { Zhang et al., 2005. }\end{array}$ \\
\hline
\end{tabular}




\subsubsection{C-2 derived Sal A analogues}

Sal A has a quick onset of action which is attributed to its rapid crossing of the blood brain barrier (approximately 1 min; Butelman et al., 2007; Schmidt et al., 2005; Hooker et al., 2008). Furthermore, Sal A has an elimination half life of $56.6 \pm 24.8 \mathrm{~min}$ in non-human primates (Schmidt et al., 2005). Sal A gets hydrolysed at C-2 position via enzyme esterases to Sal B, which is pharmacologically inactive (Chavkin et al., 2004 Ansenoff et al., 2006; Wang et al., 2008; Fig 1.6). The position C-2 is considered as an important site for the binding and activation of KOPr by Sal A (Bikbulatov et al., 2007; Yan and Roth, 2004). Additionally, this position is also implicated in improving the pharmacokinetic profile of Sal A (Prisinzano and Rothman, 2008; Munro et al., 2008). Substitution of methoxymethyl and ethoxymethyl groups at C-2 position yields compounds with increased duration of effect and greater affinity for KOPr as compared to Sal A (MOM Sal B, EOM Sal B; Munro et al., 2005; Beguin et al., 2008; Fig 1.6). Additionally, aromatic substitution at the C-2 position produces compounds with a greater affinity for MOPr than KOPr (Herkinorin; Harding et al., 2005; Tidgewell et al., 2006; Fig 1.6). The substitution of a mesylate group at this position yielded a compound which had a similar affinity but slightly higher potency for KOPr as compared to Sal A (DS1; Harding et al., 2005, Fig 1.6). One of the aims of this study was to determine behavioural anti-addiction and side-effect profiles of novel Sal A analogues. The following section describes the pharmacological profile of Sal A and selected analogues.. 


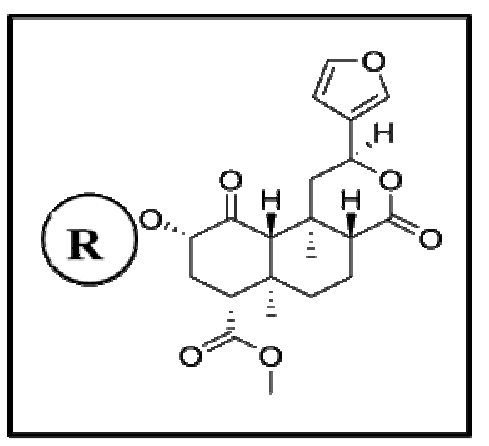

\begin{tabular}{|c|c|c|c|c|c|c|}
\hline$-\mathbf{R}$ & Compound & $\begin{array}{c}\text { Binding } \\
\text { affinity } \\
\text { at KOPr } \\
K i(\mathrm{nM})\end{array}$ & $\begin{array}{c}\text { Binding } \\
\text { affinity at } \\
\text { MOPr Ki } \\
\text { (nM) }\end{array}$ & $\begin{array}{c}\text { Potency } \\
\text { at KOPr } \\
\text { EC }_{50} \\
(\mathrm{nM})\end{array}$ & $\begin{array}{l}\text { Potency } \\
\text { at } \\
\text { MOPr } \\
\text { (nM) }\end{array}$ & Reference \\
\hline $\begin{array}{l}-\mathrm{CO}- \\
\mathrm{CH}_{3}\end{array}$ & Salvinorin A & $1.9 \pm 0.2^{\mathrm{a}}$ & $>10000$ & $\begin{array}{l}1.8 \pm 0.5^{\mathrm{c}} \\
40 \pm 10^{\mathrm{d}}\end{array}$ & & $\begin{array}{c}\text { Harding et al., } \\
2005 ; \\
\text { Tidgewell et } \\
\text { al., 2008; } \\
\text { Munro et al., } \\
2008 \\
\end{array}$ \\
\hline$-\mathrm{H}$ & Salvinorin B & $>10000$ & $>10000$ & - & & $\begin{array}{c}\text { Chavkin et al., } \\
2004\end{array}$ \\
\hline $\begin{array}{l}-\mathrm{CH}_{2-}^{-} \\
\mathrm{O}-\mathrm{CH}_{3} \\
\end{array}$ & $\begin{array}{c}\text { 2-methoxymethyl } \\
\text { Salvinorin B }\end{array}$ & $\begin{array}{l}0.6 \pm \\
0.07^{\mathrm{b}}\end{array}$ & $\mathrm{ND}$ & $0.40 \pm 0.04^{\mathrm{c}}$ & & $\begin{array}{c}\text { Munro et al., } \\
2008 \\
\end{array}$ \\
\hline $\begin{array}{c}-\mathrm{CH}_{2^{-}} \\
\mathrm{O}- \\
\mathrm{C}_{2} \mathrm{H}_{5}\end{array}$ & $\begin{array}{l}\text { 2-ethoxymethyl } \\
\text { Salvinorin B }\end{array}$ & $\begin{array}{l}0.32 \pm \\
0.02^{\mathrm{b}}\end{array}$ & ND & $0.14 \pm 0.01^{\mathrm{c}}$ & & $\begin{array}{l}\text { Munro et al., } \\
2008\end{array}$ \\
\hline $\begin{array}{l}-\mathrm{SO}_{2-}^{-} \\
\mathrm{CH}_{3} \\
\end{array}$ & DS-1 & $2.3 \pm 0.1^{\mathrm{a}}$ & $6820 \pm 660^{a}$ & $30 \pm 5^{d}$ & & $\begin{array}{l}\text { Harding et al., } \\
2005\end{array}$ \\
\hline $\begin{array}{l}-\mathrm{CO}- \\
\mathrm{C}_{6} \mathrm{H}_{5}\end{array}$ & Herkinorin & $90 \pm 2^{a}$ & $12.1 \pm 1^{\mathrm{a}}$ & $1320 \pm 150^{\mathrm{d}}$ & & $\begin{array}{c}\text { Harding et al., } \\
2005 ; \\
\text { Tidgewell et } \\
\text { al., } 2008\end{array}$ \\
\hline & Nalmefene & $\begin{array}{l}0.083^{ \pm} \\
0.0008^{\mathrm{b}}\end{array}$ & $0.24 \pm 0.006^{b}$ & $\begin{array}{c}\mathrm{EC}_{50}= \\
4.2 \pm 1.6^{\mathrm{c}} \\
\mathrm{IC}_{50}= \\
18 \pm 1.0^{\mathrm{c}}\end{array}$ & $\begin{aligned} \mathrm{IC}_{50} & = \\
13 & \pm 2.3^{\mathrm{c}}\end{aligned}$ & $\begin{array}{l}\text { Bart et al., } \\
2005\end{array}$ \\
\hline
\end{tabular}

\section{Fig.1.6. Salvinorin A, its structural analogues and nalmefene}

(Adapted from Prisinzano and Rothman, 2008) Binding affinity of Sal A, C-2 derived analogues of Sal $A$ and nalmefene for KOPr and MOPr. ${ }^{a}$ Data expressed as $\pm S D ;{ }^{b}$ Data expressed as \pm SEM; $N D=$ not defined. ${ }^{c}$ Enhancement of [35S]GTPcS binding to CHO-hKOPr; EC50 $\pm S E M(n M) .{ }^{d}[35 S] G T P-\gamma S$ functional assay carried out in stably transfected CHO cells containing DNA for hKOPr;EC50 $\pm S D n M . N D=$ not defined. 


\subsection{Mixed opioid compounds as anti-cocaine agents.}

MOPr and KOPr activation have been shown to produce opposite reward related behaviours (Pan, 1998; Smith et al., 2003; Glick et al., 1995). MOPr activation produces self-administration (Bozarth and Wise, 1981; Devine and Wise, 1994), decreases ICSS thresholds (Esposito and Kornetsky, 1977), enhances motor function (Oka and Hosoya, 1976), produces sensitization (Smith et al., 2003) and cross sensitization to the rewarding effects of cocaine (Kim et al., 2004; Smith et al., 2009). Thus, activation of MOPr produces reward related behaviours with the potential to cause addiction (Wang et al., 2004). Moreover, MOPr agonists have shown to produce place and taste preference in laboratory animals (Mucha and Herz, 1985; 1986). Activation of MOPr increases DA levels in the mesolimbic brain regions when measured using microdialysis techniques (Di Chiara and Imperato, 1988a; 1988b: Spanagel et al., 1992; Devine et al., 1993). On the other hand, KOPr activation produces opposite reward related effects (Mucha and Herz, 1985; Shippenberg and Herz, 1986; Di Chiara and Imperato, 1988a; Spanagel et al., 1992). Rats produced aversive behaviour when either MOPr was inhibited or KOPr was activated in the dorsal periaquiductal grey region (Nobre et al., 2000; Sante et al., 2000). Moreover, morphine induced place preference was blocked by KOPr agonists (Funada et al., 1993; Liang et al., 2006). Additionally, KOPr activation attenuated morphine self-administration in rats (Glick et al., 1995; Kuzmin et al., 1997). Taken together, these findings suggest the opposing role of $\mathrm{KOPr}$ and MOPr activation in reward related behaviours.

Nalmefene (REVEX; Ohmeda Pharmaceutical Products, Liberty Corner, NJ) is a longer acting structural analogue of the non-selective opioid receptor antagonist naloxone and naltrexone (June et al., 1999; Barson et al., 1989). It has a slow onset of action and has prolonged effects on morphine induced behaviours such as antinociception, locomotion and withdrawal effects in mice (Osborn et al., 2010). 
Clinical studies with nalmefene indicate that it is effective in reducing symptoms of pathological gambling (Grant et al., 2006; 2010), attenuate ethanol self-administration in laboratory animals (June et al., 1998; 2002; 2004) and humans (Mason et al., 1999; Karhuvara et al., 2007). Because of these reasons, it has been proposed for clinical use in ethanol abuse (Jupp and Lawrence 2010; Soyka and Rosner, 2010). Moreover, nalmefene is used clinically in opioid overdose (Wang et al., 1998; Barson et al., 1989). Recent opioid receptor affinity and selectivity studies show that nalmefene has a greater affinity for KOPr and MOPr than delta opioid receptors (Bart et al., 2005). The same study showed that nalmefene is a MOPr antagonist and partial KOPr agonist (Bart et al., 2005).

The reported anti-addiction properties of nalmefene (Kreek et al., 2002; 2005; Bart et al., 2005) on cocaine are required to be evaluated before any conclusions can be made on its therapeutic potential. Therefore, one of the aims of this thesis was to study the anti-cocaine effects and behavioural adverse effect profile of nalmefene in rats. 


\subsection{Aims}

KOPr agonists have been implicated in anti-addiction behaviours but have not been developed clinically because of the adverse effects associated with them (Walsh et al., 2001a; Mello and Negus, 2000). The emergence of Sal A as a novel KOPr ligand has opened a new avenue in kappa opioid research. Sal A is structurally dissimilar to the traditional KOPr agonists, yet it produces similar pharmacology at KOPr. Therefore, if Sal A produces anti-addiction behaviour like traditional KOPr agonists with fewer adverse effects, then Sal A (and its structural derivatives) could open a novel class of KOPr agonists with potential anti-addiction effects (Prisinzano and Rothman, 2008; Munro et al., 2008; Beguin et al., 2008; Wee and Koob, 2010; Prisinzano, 2008; Prevatt-Smith and Prisinzano, 2010).

With this as the central hypothesis of this thesis, Sal A, its novel analogues (MOM Sal B, EOM Sal B, herkinorin, DS1) and MOPr antagonist/ partial KOPr agonist nalmefene were pharmacologically screened for their anti-addiction effects using the cocaine prime induced reinstatement model. Furthermore, the anti-cocaine profile of those $\mathrm{KOPr}$ compounds were determined which attenuated drug-seeking in a selective manner without producing motor suppression by themselves. This was performed by using cocaine induced enhancement and sensitization to motor function in rats.

The next aim of this thesis was to test for the selectivity of the attenuation of cocaine induced reinstatement. This was done by measuring sucrose reinforcement (attenuation in operant reinforcement) and cocaine induced hyperactivity in selfadministering animals (motor suppression during reinstatement test). Another aim of this thesis was to evaluate the behavioural adverse effects of the KOPr agonists which were successful at attenuating cocaine reinstatement. This was achieved by using spontaneous open field activity (motor suppression), conditioned taste aversion (aversion) and forced swim test (depression) in rats. 
The last part of this thesis applies behavioural and in vitro models to better understand the mechanism of KOPr mediated depression. This was achieved by investigating the effects of serotonin transporter (SERT) blockade on KOPr agonists induced depression and effect of KOPr activation on in vitro SERT function. 
Chapter 2. Anti-addiction profile of KOPr agonists. 


\subsection{Introduction}

\subsubsection{Cocaine prime induced reinstatement model}

The phenomenon of reinstatement of drug seeking in animals has been shown to mimic human conditions (Sinha et al., 1999; Littleton, 2000; Shalev et al., 2002; Shaham et al., 2003). Presentation of stimuli such as drug primes, environmental cues and stressors have all been shown to initiate drug-seeking in both animals and humans (Ludwig et al., 1974; De Wit and Stewart, 1981; 1983; Jaffe et al., 1989; Koob et al., 1996; McFarland and Ettenberg, 1997; Erb et al., 1996; Sinha et al., 1999; 2001; Weiss et al., 2001). Due to these reasons, pre-clinical models have been extensively used to study the neurobiology of drug addiction (Markau et al., 1993; Koob and La Moal, 2001, 2005; Koob, 2006; Olmstead, 2006). The reinstatement of cocaine self-administration model mimics the potential to relapse back to drug use following a period of extinction in laboratory animals (Stewart, 2000; Epstein et al., 2006; Shalev et al., 2002; Fuchs et al., 1998; Shaham et al., 2003). Therefore, this paradigm has been used extensively for the pharmacological evaluation of potential anti-addiction compounds (Schenk et al., 1999a; 1999b; Beardsley et al., 2005; 2010).

Reinstatement of drug-seeking via self-administration training has been performed pre-clinically using between session (Stretch et al., 1971), within session (de Wit and Stewart, 1981; 1983) and between-within session models (Tran-Nguyen et al., 1998). In between session reinstatement, animals undergo self-administration, extinction training sessions and reinstatement test on separate days (Stretch et al., 1971; Stewart, 2000). The within session reinstatement model animals self-administer cocaine for 1-2 $\mathrm{hr}$ followed by the extinction training (for 3-4 $\mathrm{hr}$ ) and then testing for reinstatement with all the procedures performed in a single day (de Wit and Stewart, 1981; 1984). In between-within reinstatement test, animals are trained to self-administer 
cocaine on different days, whereas extinction and reinstatement tests are performed on the same day (Tran-Nguyen et al., 1998; Shaham et al., 2003).

In the current study, the within session reinstatement paradigm was used (Worley et al., 1994; Schenk et al., 1999b; 2000a). This paradigm mimics the potential of priming injection of cocaine to reinstate cocaine self-administration in rats which were previously self-administering cocaine and were subjected to short period of extinction training (Stewart, 2000; Epstein et al., 2006). An advantage of this model is that it can be used to screen the effects of acute pharmacological exposure on cocaine seeking behaviour (Schenk et al., 1999b; 2000a; 2001b; Schenk, 2000; Schenk and Partridge, 2001; Morani et al., 2009). Furthermore, traditional KOPr agonists, U69593, $\mathrm{U} 50488 \mathrm{H}$ and spiradoline have all been shown to attenuate reinstatement of drugseeking using this paradigm (Schenk et al. 1999b, 2000a; Morani et al., 2009). Therefore, in order to characterize the effects of KOPr compounds on cocaine seeking behaviour, we performed an initial preliminary screening using the within session reinstatement paradigm in rats.

\subsubsection{Cocaine induced enhancement in motor activity}

Experimenter administered cocaine has been shown to produce increases in motor function in laboratory animals (Post and Contel, 1983; Kalivas and Stewart, 1991). A robust enhancement in motor function is also observed in animals with prior cocaine experience in which an acute challenge of injected cocaine reveals motor sensitization (Post and Contel, 1983; Pierce and Kalivas, 1997; Vanderschuren and Kalivas, 2000). This sensitized response persists even after a period of prolonged abstinence (Robinson and Berridge, 1993; 2001; 2003), thus indicating the long lasting alterations in central neuronal circuits following intermittent cocaine exposures (Robinson and Berridge, 1993; 2000; 2003; 2008; Pierce and Kalivas, 1997). Expression and development of behavioral sensitization is a paradigm that highlights 
cocaine's ability to alter neural circuits underlying its psychomotor effects (Robinson and Berridge, 1993; 2001; 2003; 2008; Kalivas et al., 1998; O’Brian et al., 1992; Todtenkopff et al., 2002; Lambert et al., 2006). Therefore, increasing our understanding of the factors which attenuate the expression and development of cocaine induced behavioural sensitization could prove useful in designing therapeutics for cocaine addiction (Pierce and Kalivas, 1997; Robinson and Berridge, 2003). Recently, Chartoff and colleagues (2008) showed that acute exposure to Sal A attenuates hyperactivity to a single injection of cocaine in a similar way to traditional KOPr agonists. In order to extend the findings of Chartoff et al. (2008), this study characterizes the anti-cocaine profile of Sal A and another novel KOPr agonist (DS1) on cocaine induced hyperactivity and expression of behavioural sensitization in rats. As forward locomotion during sensitization experiments does not augment with cocaine produced stereotypy (Ushijima et al., 1995; Post et al., 1987), we further analysed the effect of Sal A and DS1 on cocaine produced stereotypic counts in sensitized rats.

\subsubsection{Aims}

The novel non-nitrogenous structure of Sal A has led to the synthesis of many KOPr agonists with unknown pharmacology (Prisinzano and Rothman, 2008; Munro et al., 2008; Section 1.6; Chapter 1). This chapter aims to determine if these novel compounds have anti-addiction pharmacology. To do this we will:

1. Screen the effect of novel KOPr compounds Sal A, DS1, MOM Sal B, EOM Sal B, herkinorin and MOPr antagonist/partial KOPr agonist, nalmefene on cocaine prime induced drug-seeking in rats.

2. Determine if the effects seen are in fact KOPr mediated. This will be performed by measuring the behavioural effect of pre-treatment with selective KOPr antagonist (nor-BNI; Portoghese et al., 1987) on KOPr agonist mediated attenuation in drug-seeking. 
3. Compounds shown to attenuate drug-seeking in a specific manner without suppressing motor function by themselves (Chapter 3) will be further characterized behaviourally to determine their effects on cocaine induced enhancement in motor function (Heidbreder et al., 1993; Collins et al., 2001a; 2001b). This will be determined experimentally by measuring changes in cocaine induced hyperactivity, expression of behavioural sensitization and cocaine produced stereotypy in rats.

\subsection{Methods}

\subsubsection{Subjects}

Male Sprague-Dawley rats weighing 325-350 g (for reinstatement test; one per cage) and 200-250 g (for cocaine induced hyperactivity and behavioural sensitization tests; two per cage) were housed in a temperature $\left(19-21^{\circ} \mathrm{C}\right)$ and humidity $(55 \%$ relative humidity) controlled room at the animal facility in the School of Psychology, Victoria University of Wellington. Lights were maintained on a 12:12 hr cycle with lights on at 0700. Animals had free access to food and water except during experimental sessions. All rats were housed in the animal facility five days prior the commencement of experiments and were handled daily to reduce handling stress. All experimental procedures were reviewed and approved by the Animal Ethics Committee of Victoria University of Wellington.

\subsubsection{Drugs}

Cocaine $\mathrm{HCl}$ (Merck Pharmaceuticals, Palmerston North, New Zealand), norBNI and nalmefene (Sigma-Aldrich, St. Louis, MO, USA) were dissolved in physiological saline. Sal A, MOM Sal B, EOM Sal B, DS1 and herkinorin (gifts from Dr. Thomas E. Prisinzano, University of Kansas) were suspended in 75\% DMSO. 
Subcutaneous (sc) or intraperitoneal (ip) injections were in a volume of $1 \mathrm{ml} / \mathrm{kg}$. IV infusions were given in the volume of $100 \mu \mathrm{l}$. All drug weights refer to the salt.

\subsubsection{Cocaine prime induced reinstatement test}

\subsubsection{Surgery}

The surgery was performed by Mr. Caleb Carati, Mr. Alex Howard and Mr. Alex Crowther. Under deep anaesthesia produced by ketamine/xylazine (90/9 mg/kg, ip; Provet, Palmerston North, New Zealand), the right jugular vein was isolated and the catheter was inserted. The distal end (22 gauge stainless steel tubing) was passed sc to an exposed portion of the skull where it was fixed to embedded jeweller's screws with dental acrylic. The catheters were infused daily with $0.1 \mathrm{~mL}$ of a sterile saline solution containing heparin (30.0 U/mL; Provet, Palmerston North), penicillin G Potassium (250,000 U/mL; Provet, Palmerston North) and streptokinase (8000 U/mL; Health Care Logistics, Auckland) to prevent infection and the formation of clots and fibroids. The rats were allowed five days post surgery to recover.

\subsubsection{Apparatus}

Self-administration training and reinstatement testing were carried out in humidity $(55 \%)$ and temperature $\left(19-21^{\circ} \mathrm{C}\right)$ controlled environment in standard operant chambers (Med Associates, ENV-001) equipped with 2 levers. Depression of one lever (the active lever) resulted in a $0.1 \mathrm{~mL}$ iv infusion of cocaine $\mathrm{HCl}$ dissolved in sterile physiological saline containing heparin $(3.0 \mathrm{U} / \mathrm{mL})$. Infusions were of $12 \mathrm{sec}$ duration. Coincident with drug delivery was the illumination of a stimulus light located directly above the active lever. This stimulus light remained illuminated throughout each $12 \mathrm{sec}$ infusion. Depression of the other lever (the inactive lever) was without programmed consequence. 
Rats were maintained in the animal colony except during testing. Immediately prior to each daily self-administration session, the catheter lines were infused with 0.1 $\mathrm{mL}$ of heparin-penicillin-streptokinase solution. The stainless steel catheter was connected to a length of microbore tubing and connected to the syringe. At the end of each session, the lines were again infused with $0.1 \mathrm{~mL}$ of the heparin-penicillinstreptokinase solution, the stainless steel tubing was plugged and the animal was returned to its home cage. Drug delivery and data acquisition were controlled using Med Associates software (St. Albans, VA, USA). Cocaine deliveries were made via mechanical pumps (Razel, Model A with $1.0 \mathrm{rpm}$ motor equipped with $20 \mathrm{~mL}$ syringe).

\subsubsection{Cocaine self-administration training}

The cocaine self-administration protocols we followed have been described by Worley et al, (1994), Schenk et al, (1999b) and Morani et al., (2009). Rats were initially trained to self-administer cocaine using a FR-1 schedule of reinforcement (pressing the active lever once delivered a single infusion of cocaine, $0.5 \mathrm{mg} / \mathrm{kg} /$ infusion) for $2 \mathrm{hr}$. The self-administration training was performed in the dark using light proof boxes. Once stable responding was achieved (at least 20 infusions per $2 \mathrm{hr}$ session for 3 consecutive days), they were upgraded to FR-5 schedule of reinforcement, where in pressing the active lever five times resulted in a single infusion of cocaine $(0.5$ $\mathrm{mg} / \mathrm{kg} /$ infusion). Self-administration was considered to be acquired when there was less than $20 \%$ variation in responding for three consecutive days.

\subsubsection{Cocaine prime induced reinstatement test.}

Once responding on the FR-5 schedule was stable, the effect of KOPr agonist (Sal A, MOM Sal B, EOM Sal B, DS1, herkinorin and nalmefene) administration on drug-seeking produced by a priming injection of cocaine was measured. This test was conducted in a single day and consisted of three phases. The first phase comprised a one $\mathrm{hr}$ period of cocaine self-administration $(0.5 \mathrm{mg} / \mathrm{kg} /$ infusion, FR-5 schedule of 
reinforcement) in which the light stimulus was paired with cocaine infusions. After the one hr self-administration period, the cocaine solution was replaced with heparinised saline and responding was reinforced with the vehicle infusion (FR-5 schedule of reinforcement) coupled with illumination of the light stimulus. Saline reinforced responding was measured during the second phase which was of $3 \mathrm{hr}$ duration. By the end of the $3 \mathrm{rd} \mathrm{hr}$, animals which showed less than $20 \%$ of responding as compared to phase 1 were subjected to the reinstatement test. At the beginning of this third phase, separate groups of rats $(n=5-10)$ received an injection of either vehicle $(0.9 \%$ saline or $75 \%$ DMSO) or KOPr agonists Sal A (0.1, 0.3 and $1.0 \mathrm{mg} / \mathrm{kg}$, ip); MOM Sal B (0.03, 0.1 and $0.3 \mathrm{mg} / \mathrm{kg}, \mathrm{ip})$; EOM Sal B (0.03, 0.1 and $0.3 \mathrm{mg} / \mathrm{kg}$, ip); DS1 (0.1, 0.3 and 1.0 $\mathrm{mg} / \mathrm{kg}$, ip); herkinorin $(0.1,0.3$ and $1.0 \mathrm{mg} / \mathrm{kg}$, ip) or nalmefene $(0.1,0.3$ and $1.0 \mathrm{mg} / \mathrm{kg}$, sc). These injections were administered either 5 (Sal A, MOM Sal B, EOM Sal B, herkinorin), 15 (nalmefene) or 45 (DS1) min prior to an injection of cocaine (20 mg/kg, ip). This was followed by measuring saline reinforced responding at FR-5 schedule of reinforcement with an infusion of vehicle solution (heparinised saline) and illumination of the light stimulus. Responding was measured for $1 \mathrm{hr}$ following the cocaine injection. Operant lever press responding observed during the three phases of the reinstatement test is shown in Fig 2.1.

The vehicle used to suspend Sal A and its derivatives was selected based on previous studies showing that $75 \%$ DMSO was well tolerated by rats (WillmoreFordham et al., 2007; Baker et al., 2009; Chartoff et al., 2008; Morani et al., 2009). Sal A, MOM Sal B and EOM Sal B have a quick onset of action via the ip route of administration (Hooker et al., 2009a; Baker et al., 2009; Wang et al., 2008). Also, previous studies with herkinorin suggest a quick onset of action in vivo (Butelman et al., 2008). Therefore, the pre-treatment time was chosen as 5 min for Sal A, MOM Sal B, EOM Sal B and herkinorin. The route of administration (sc) and pre-treatment time (15 
min) for nalmefene was selected based on previously published reports (June et al., 1998).

\subsubsection{KOPr antagonist reversal experiment}

The effect of 30 min pre-treatment with selective KOPr antagonist, nor-BNI (2 $\mathrm{mg} / \mathrm{kg}$, ip) on attenuation of cocaine prime induced drug-seeking by the KOPr agonists was also performed. This experiment was performed in order to verify that attenuation of reinstatement behaviour by the novel KOPr compounds was mediated via KOPr activation. Also, this experiment was performed on KOPr compounds using a minimum effective dose $(0.3 \mathrm{mg} / \mathrm{kg})$ at which they attenuated drug-seeking behaviour in a specific manner (Sal A, DS1, nalmefene; Chapter 3). The experimental conditions were similar to the reinstatement test, except that rats $(n=5-10$ for each group) received an injection of nor-BNI (0, $2 \mathrm{mg} / \mathrm{kg}$, ip) $30 \mathrm{~min}$ prior to the exposure to $\mathrm{Sal} \mathrm{A}(0.3 \mathrm{mg} / \mathrm{kg}$, ip), DS1 (0.3 $\mathrm{mg} / \mathrm{kg}$, ip) or nalmefene $(0.3 \mathrm{mg} / \mathrm{kg}, \mathrm{sc})$. Saline-reinforced responding was recorded following the priming injection of cocaine $(20 \mathrm{mg} / \mathrm{kg}$, ip). The dose $(2 \mathrm{mg} / \mathrm{kg})$, route of administration (ip) and pre-treatment time $(30 \mathrm{~min})$ for nor-BNI was also selected based on previous reports (Xu et al., 2006). 


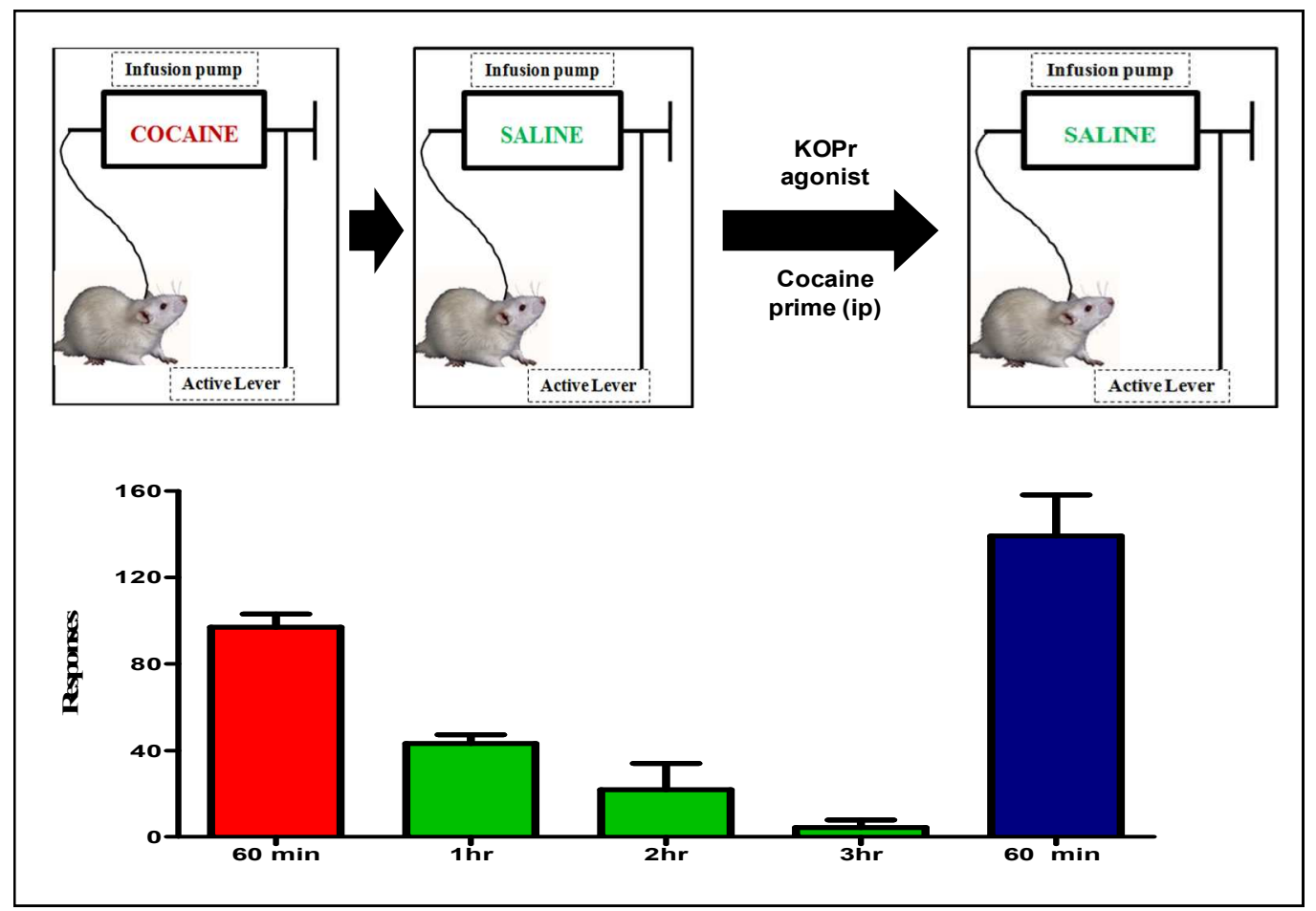

\section{Fig 2.1. Cocaine prime induced reinstatement test.}

Animals previously self-administering cocaine stably at a FR-5 schedule of reinforcement were subjected to the reinstatement test. During the first phase, rats were allowed to self administer cocaine (0.5 mg/ml/infusion) for $1 \mathrm{hr}$. Corresponding active lever presses were observed to be high. During the second phase, cocaine was replaced by saline in the infusion pump. This resulted in extinguished lever press responding by the end of the $3^{r d} h r$. Prior to the beginning of the third phase, animals received either KOPr agonist or corresponding vehicle and a single priming injection of cocaine $(20 \mathrm{mg} / \mathrm{kg}$, ip) and saline reinforced active lever responding was measured for $1 \mathrm{hr}$. In the above figure, the high rate of responding during the third phase is due to the priming injection of cocaine $(20 \mathrm{mg} / \mathrm{kg})$ as the infusion pump contained only heparinised saline. Therefore, this reinstatement is called cocaine prime induced reinstatement of cocaine seeking following a short period of extinction $(n=8)$. 


\subsubsection{Cocaine induced hyperactivity and behavioural sensitization}

Cocaine induced hyperactivity and behavioural sensitization tests were performed to further explore the anti-cocaine effects of novel KOPr agonists which showed attenuation of drug-seeking behaviour in a specific manner without suppressing motor function (Chapter 3).

\subsubsection{Apparatus}

Eight open field chambers (Med Associates, ENV-520) equipped with two banks of sixteen photocells on each wall were used to measure horizontal and vertical locomotion. The open field boxes were interfaced with an adjacent microcomputer. Stereotypic counts during the sensitization experiments were determined by measuring repetitive beam breaks obtained from the activity monitoring software (Med Associates). Testing was conducted in the dark between 1000 and $1600 \mathrm{hr}$. White noise was continually present to mask extraneous disturbances. The floor and the sides of the test chamber were cleaned before and after testing each animal using Virkon "S" disinfectant (Southern Veterinary Supplies, Palmerston North, New Zealand).

\subsubsection{Procedure}

\section{A. Cocaine induced hyperactivity in drug naive rats.}

For this study we modified the methods of Frankowska et al., (2009) and Xu et al., (2010). On the test day, drug naive rats received an injection of either vehicle $(75 \%$ DMSO) or KOPr compound (Sal A, $0.3 \mathrm{mg} / \mathrm{kg}$; DS1, $0.3 \mathrm{mg} / \mathrm{kg}$ ). Five minutes later (or 45 min following DS1 injection) this was followed by an injection of either $0.9 \%$ saline $(1 \mathrm{~mL} / \mathrm{kg})$ or cocaine $(20 \mathrm{mg} / \mathrm{kg}$, ip). Immediately following the second injection, total activity, a compilation of horizontal and vertical activity was measured every 5 min for $60 \mathrm{~min}$. 


\section{B. Expression of cocaine sensitization and stereotypy.}

For this study we modified the methods described by Frankowska et al., (2009) and $\mathrm{Xu}$ et al., (2010). Rats received a single injection of either $0.9 \%$ saline $(1 \mathrm{~mL} / \mathrm{kg})$ or cocaine (20 mg/kg, ip) for 5 consecutive days (day 1-5) in their home cages. On days 69 the animals remained drug-free in their home cages. On the test day (day 10), animals first received KOPr compounds (Sal A, DS1) or vehicle (75\% DMSO) followed by a single injection of cocaine $(20 \mathrm{mg} / \mathrm{kg}$, ip). Locomotor activity (total ambulatory counts) and cocaine produced stereotypy was measured at $5 \mathrm{~min}$ intervals for $60 \mathrm{~min}$. 
$\underline{\text { Tab 2.1 Experimental groups for cocaine induced hyperactivity. }}$

\begin{tabular}{|c|c|}
\hline Acute Pre-treatment (ip) & Challenge injection (ip) \\
\hline Vehicle & Saline $(1 \mathrm{~mL} / \mathrm{kg})$ \\
\hline Sal A & Saline $(1 \mathrm{~mL} / \mathrm{kg})$ \\
\hline DS1 & Saline $(1 \mathrm{~mL} / \mathrm{kg})$ \\
\hline Vehicle & Cocaine $(20 \mathrm{mg} / \mathrm{kg})$ \\
\hline Sal A & Cocaine $(20 \mathrm{mg} / \mathrm{kg})$ \\
\hline DS1 & Cocaine $(20 \mathrm{mg} / \mathrm{kg})$ \\
\hline
\end{tabular}

Vehicle (75\% DMSO; $1 \mathrm{~mL} / \mathrm{kg}) ;$ Sal A (0.3 mg/kg), DS1 (0.3 mg/kg)

Tab 2.2 Experimental groups for cocaine behavioural sensitization.

\begin{tabular}{|c|c|c|}
\hline $\begin{array}{c}\text { Pre-treatment } \\
\text { (day 1-5; ip) }\end{array}$ & $\begin{array}{c}\text { Acute treatment } \\
\text { (day 10; ip) }\end{array}$ & $\begin{array}{c}\text { Challenge injection } \\
\text { (day 10; ip) }\end{array}$ \\
\hline Saline $(1 \mathrm{~mL} / \mathrm{kg})$ & Vehicle & Cocaine $(20 \mathrm{mg} / \mathrm{kg})$ \\
\hline Saline $(1 \mathrm{~mL} / \mathrm{kg})$ & Sal A & Cocaine $(20 \mathrm{mg} / \mathrm{kg})$ \\
\hline Saline $(1 \mathrm{~mL} / \mathrm{kg})$ & DS1 & Cocaine $(20 \mathrm{mg} / \mathrm{kg})$ \\
\hline Cocaine $(20 \mathrm{mg} / \mathrm{kg})$ & Vehicle & Cocaine $(20 \mathrm{mg} / \mathrm{kg})$ \\
\hline Cocaine $(20 \mathrm{mg} / \mathrm{kg})$ & Sal A & Cocaine $(20 \mathrm{mg} / \mathrm{kg})$ \\
\hline Cocaine $(20 \mathrm{mg} / \mathrm{kg})$ & DS1 & Cocaine $(20 \mathrm{mg} / \mathrm{kg})$ \\
\hline
\end{tabular}

Vehicle (75\% DMSO; $1 \mathrm{~mL} / \mathrm{kg}) ;$ Sal A (0.3 mg/kg), DS1 (0.3 mg/kg) 


\subsubsection{Statistical analysis}

Data analyses were performed by using Prism Graphpad software (Version 5.0, San Diego, CA). Statistical analysis for cocaine induced locomotion and cocaine sensitization studies (for total ambulatory counts) were performed using one-way ANOVA followed by Tukey post-hoc tests. Further statistical analysis was performed using two-way ANOVA (treatment $\mathrm{x}$ time) with repeated measures on time for cocaine induced hyperactivity, expression of sensitization and cocaine induced stereotypic counts. A Bonferroni post hoc test was also performed. The number of active lever responses during phase 3 of the reinstatement phase is shown as mean +SEM. For cocaine induced reinstatement tests separate one-way ANOVA was performed followed by Tukey post-hoc test (Morani et al., 2009). Student t-tests were performed on the data from nor-BNI reversal experiments. 


\section{$\underline{\text { 2.3. Results }}$}

\subsubsection{Effect of Sal A on cocaine prime induced reinstatement.}

The effect of Sal A $(0,0.1,0.3,1.0 \mathrm{mg} / \mathrm{kg}, \mathrm{ip})$ pre-treatment on cocaine prime $(20 \mathrm{mg} / \mathrm{kg}$, ip) induced reinstatement in rats is shown in Fig 2.2. Statistical analysis showed that pre-treatment with Sal A $(0.3,1.0 \mathrm{mg} / \mathrm{kg})$ significantly attenuated cocaine prime induced reinstatement $[\mathrm{F}(3,23)=79.33, \mathrm{p}<0.0001$; Fig $2.2 \mathrm{a}]$ in a dose dependent manner. This attenuation was not seen in animals pre-treated with the KOPr antagonist nor-BNI (0, $2.0 \mathrm{mg} / \mathrm{kg}$, ip) $(\mathrm{p}<0.001$; Fig. $2.2 \mathrm{~b})$. 
(a)

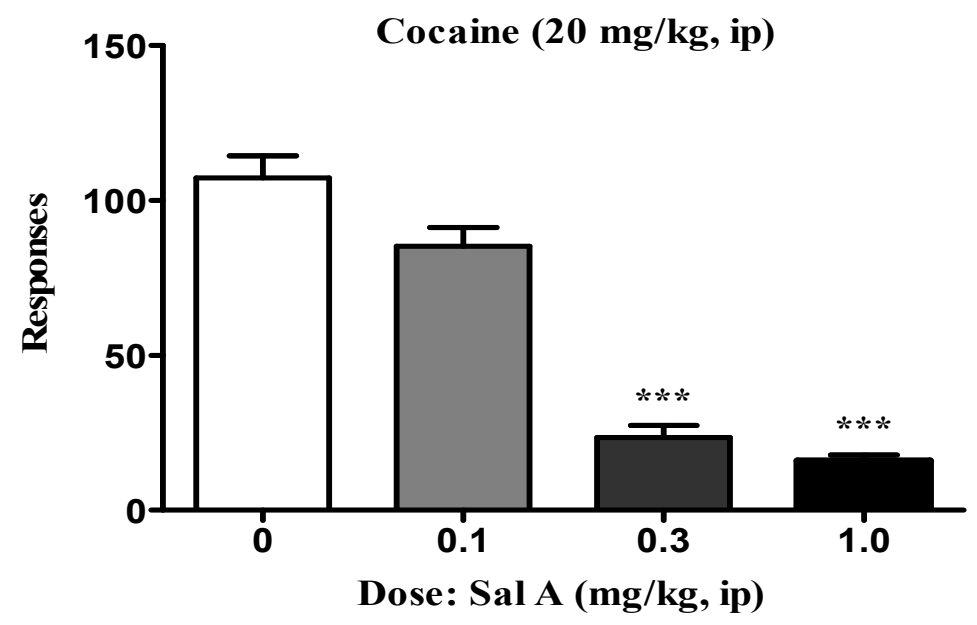

(b)

Sal A (0.3 mg/kg, ip)

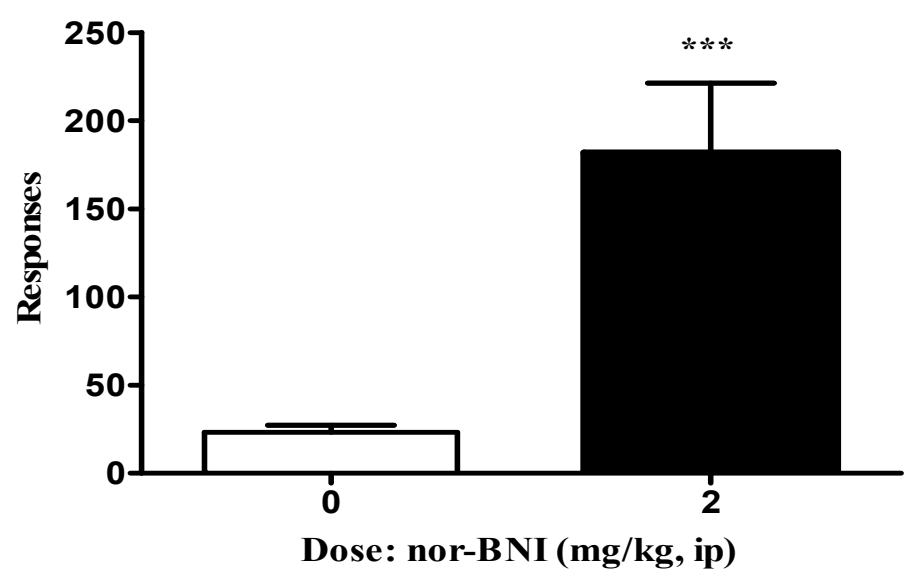

\section{Fig. 2.2 Effect of Sal A on cocaine prime induced reinstatement}

Symbols indicate active lever press responses +SEM produced during phase 3 of the reinstatement test. (a) Rats which had shown extinguished saline reinforcement during the extinction phase were initially treated with different doses of Sal A $(0,0.1,0.3$ and $1.0 \mathrm{mg} / \mathrm{kg}$ ) followed by a priming injection of cocaine $(20 \mathrm{mg} / \mathrm{kg})$ and saline reinforced responses were recorded for a period of $60 \mathrm{~min} . *_{* *}^{*}<0.001$, data were compared with vehicle treated group (0 mg/kg). One-way ANOVA followed by Tukey post hoc test. $n=6-7$ for each group (b) For nor$B N I$ reversal experiments, animals were first treated with nor-BNI $(0,2.0 \mathrm{mg} / \mathrm{kg})$ followed by Sal A $(0.3 \mathrm{mg} / \mathrm{kg})$ and cocaine $(20 \mathrm{mg} / \mathrm{kg})$ injections. ${ }^{* * *} p<0.001$, Student t-tests. $n=5-7$ for each group. 


\subsubsection{Effect of Sal A on cocaine induced hyperactivity (drug naïve animals)}

The effect of Sal A $(0,0.3 \mathrm{mg} / \mathrm{kg}$, ip) pre-treatment on cocaine $(0,20 \mathrm{mg} / \mathrm{kg}$, ip) induced hyperactivity in rats is shown in Fig 2.3. A significant increase in the locomotion activity was observed in animals pre-treated with vehicle/cocaine as compared to those which were treated with vehicle/saline $(\mathrm{p}<0.01)$ demonstrating cocaine induced hyperactivity in rats $[\mathrm{F}(3,22)=22.2, \mathrm{P}<0.0001$; Fig. 2.3a]. Post hoc analysis indicated no significant difference between Sal A and vehicle pre-treatment on the locomotor activity produced following the saline $(\mathrm{P}>0.05)$ or cocaine $(\mathrm{P}>0.05)$ injection (Fig 2.3). A non-significant trend towards an increase in total activity was observed in Sal A/cocaine treated rats as compared to vehicle/cocaine treated rats $(\mathrm{P}>$ 0.05). Further time course comparison revealed a significant treatment $[\mathrm{F}(3,364)=$ 101.4, $\mathrm{P}<0.0001]$ and interaction $[\mathrm{F}(33,364)=3.28, \mathrm{P}<0.0001]$ effect $($ Fig 2.3b). Post-hoc tests showed a significant increase in activity for Sal A/Coc treated group vs. Veh/Coc treated group of animals 5, 10 and 15 min following cocaine injection $(\mathrm{P}<$ $0.05)$. 
(a)

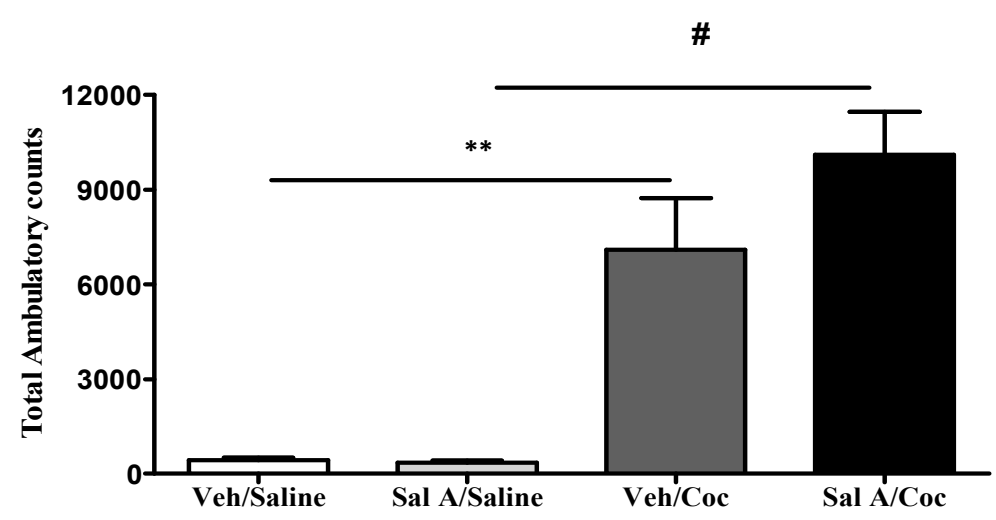

(b)

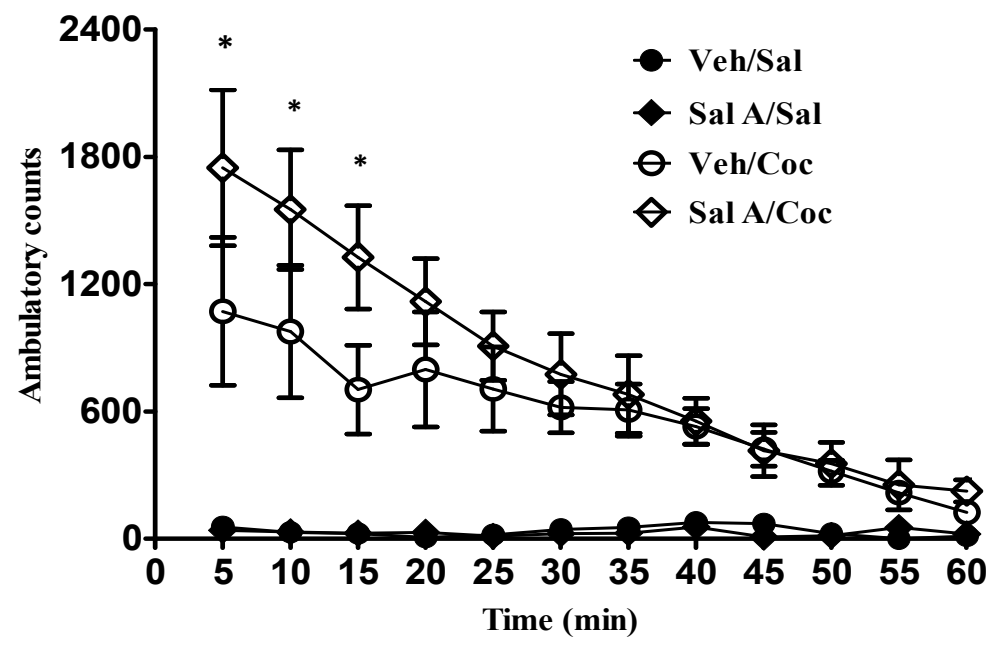

Fig. 2.3 Effect of Sal A on cocaine induced hyperactivity (drug naive animals).

Drug naive rats were initially injected with either vehicle (75\% DMSO) or Sal A (0.3 mg/kg) followed by saline $(1 \mathrm{~mL} / \mathrm{kg}$ ) or cocaine (Coc; $20 \mathrm{mg} / \mathrm{kg}$ ) and locomotion activity was measured for $60 \mathrm{~min}$. (a) Figures represents mean total activity $+S E M$. ${ }^{* *} p<0.01$, data compared with Vehicle/Saline treated group, $\# p<0.01$, data compared with Sal A/Saline treated group. Oneway ANOVA followed by Tukey post hoc test. (b) Activity counts ( \pm SEM) measured after 5 min interval. ${ }^{*} p<0.05$, data compared with Veh/Coc treated group. Repeated measures two-way ANOVA followed by Bonferroni post-hoc test. $n=6-7$ for each group. 


\subsubsection{Effect of Sal A on expression of cocaine sensitization and stereotypy.}

The effect of Sal A $(0,0.3 \mathrm{mg} / \mathrm{kg}$, ip $)$ pre-treatment on expression of cocaine behavioural sensitization in rats is shown in Fig 2.4. Animals were pre-treated with cocaine on days 1-5 and then on day 10 exposed to vehicle followed by a cocaine injection, showed a significant increase in locomotion as compared to the group of animals which received saline from day 1-5, demonstrating the expression of cocaineinduced sensitization $[\mathrm{F}(3,23)=4.45, \mathrm{P}=0.013$; Fig. 2.4a]. Post-hoc tests revealed no measurable difference in activity between animals exposed to Sal A $(0.3 \mathrm{mg} / \mathrm{kg})$ on the test day than those receiving either saline or cocaine from day 1-5 $(\mathrm{P}>0.05$; Fig. 2.4a). However, a further time course analysis showed a significant treatment $[\mathrm{F}(3,276)=$ 23.18, $\mathrm{P}<0.0001]$ and interaction $[\mathrm{F}(33,276)=3.61, \mathrm{P}<0.0001]$ effect (Fig. 2.4b). Post hoc tests showed a significant reduction in locomotion activity in Sal A pre-treated rats vs. vehicle treated rats at 5, 10, 15 and 20 min following a single cocaine injection $(\mathrm{P}<0.05 ;$ Fig.2.4b). Time course analysis on cocaine induced stereotypic counts showed significant treatment effect $[\mathrm{F}(3,276)=3.99, \mathrm{P}<0.01]$ but no interaction effect $[\mathrm{F}(33$, $276)=1.2, \mathrm{P}=0.24]$. Bonferroni post-hoc tests showed no significant effect on cocaine induced stereotypic counts following Sal A pre-treatment in cocaine sensitized animals $(\mathrm{P}>0.05 ;$ Fig 2.4c). Sal A pre-treatment however showed a trend towards an increase in cocaine produced stereotypic counts in sensitized animals 5, 10 and 15 min post cocaine treatment compared to vehicle treated animals $(\mathrm{P}>0.05 ;$ Fig $2.4 \mathrm{c})$. 
(a)

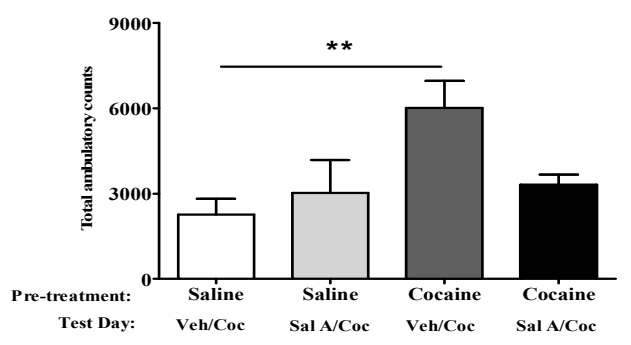

(b)

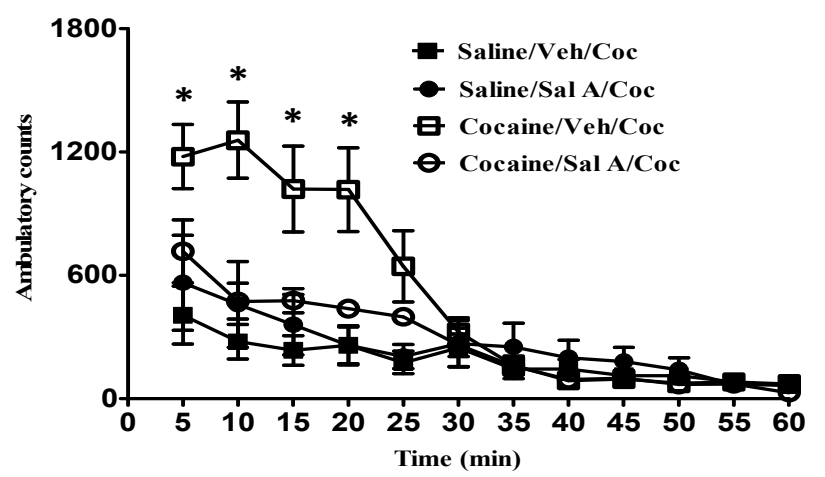

(c)

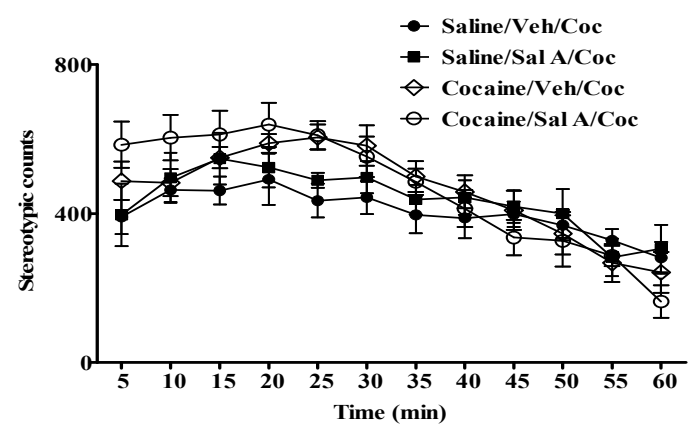

Fig. 2.4 Effect of Sal A on expression of cocaine sensitization and

\section{stereotypy.}

Rats were injected with saline (1 mL/kg) or cocaine $(20 \mathrm{mg} / \mathrm{kg})$ for 5 consecutive days. Animals remained drug free from day 6-9. On day 10, rats were injected with either vehicle (Veh, 75\% DMSO) or Sal A (0.3 mg/kg) and 5 min later were injected with cocaine (Coc, $20 \mathrm{mg} / \mathrm{kg}$ ) and activity was measured for $60 \mathrm{~min}$. (a) Data expressed as mean total ambulations $(+S E M) .{ }^{*} p<0.01$, data compared with Saline/Veh/Coc treated group. One-way ANOVA followed by Tukey test. (b) Time course measurement of mean ( \pm SEM) of locomotion activity over a period of 5 min interval. ${ }^{*} p<0.05$, vs. Cocaine/Sal A/Coc treated group. Repeated measures two-way ANOVA followed by Bonferroni post hoc test. (c) Time course measurement of mean ( \pm SEM) stereotypic counts every $5 \mathrm{~min}$ for $60 \mathrm{~min}$. Repeated measures two-way ANOVA followed by Bonferroni post hoc test. $n=6-8$ for each group. 


\subsubsection{Preliminary study to determine pre-treatment time for DS1}

Initial studies were carried out to determine the time taken for DS1 to have an effect on laboratory rat behaviour. This was ascertained by measuring the effect of DS1 on cocaine induced hyperactivity. Three separate groups of drug naive rats were pretreated with DS1 $(0.3 \mathrm{mg} / \mathrm{kg}$, ip) for 5,30 or $45 \mathrm{~min}$ prior to the injection of cocaine (20 $\mathrm{mg} / \mathrm{kg}$ ) and total ambulatory counts as well as activity after every $5 \mathrm{~min}$ interval was measured for $1 \mathrm{hr}$ (Fig. 2.5). The results indicate that DS1, when injected 5 min or 30 min prior to cocaine, had no significant effect on total activity compared to the vehicle controls (75\% DMSO; $1 \mathrm{~mL} / \mathrm{kg}$, ip). However, a significant suppression in cocaine induced hyperactivity was observed when DS1 was injected 45 min prior to cocaine exposure $[\mathrm{F}(3,14)=5.28, \mathrm{p}<0.012$; Fig. $2.5 \mathrm{a}]$. A further time course analysis showed that DS1 (administered $45 \mathrm{~min}$ prior to cocaine) significantly attenuated locomotor activity at 5, 10 and 20 min following cocaine $(p<0.05)$ when compared to vehicle pretreated controls $[\mathrm{F}(3,168)=23.64, \mathrm{P}<0.0001 ;$ Fig. $2.5 \mathrm{~b}]$. 
(a)

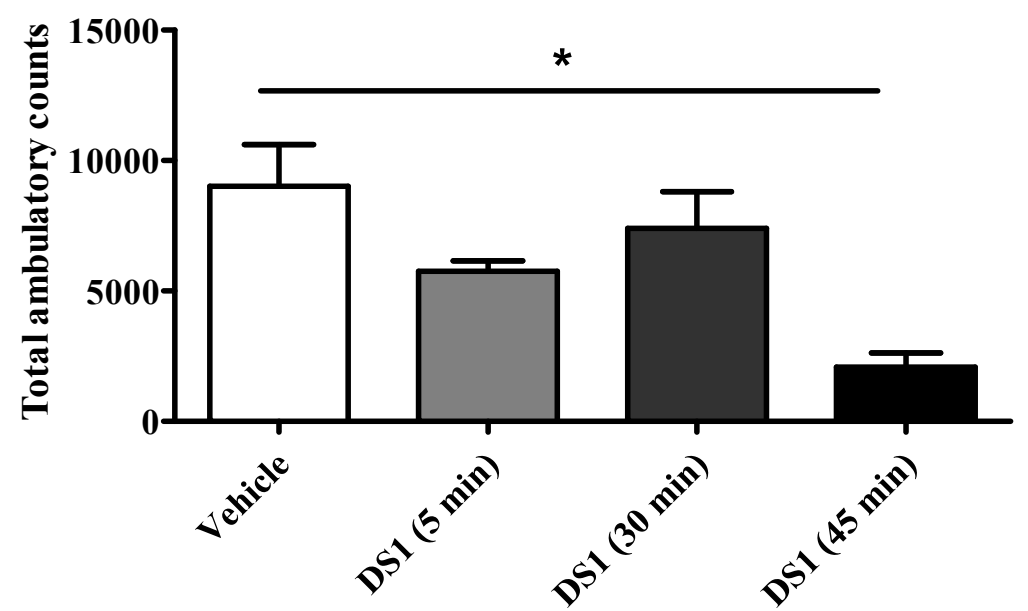

(b)

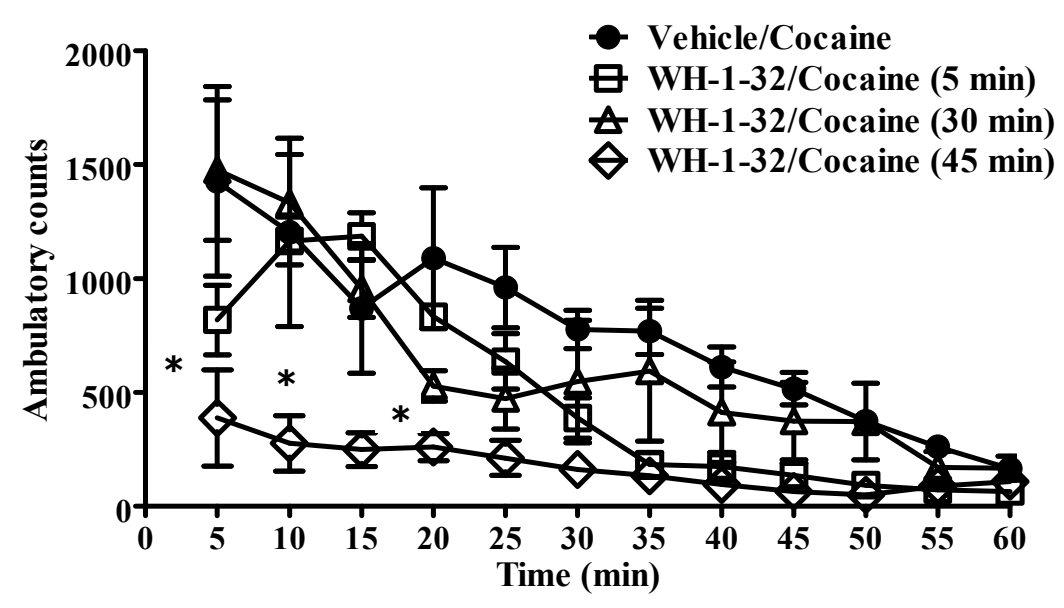

Fig. 2.5. Preliminary study to determine pre-treatment time for DS1.

Rats initially received vehicle (75\% DMSO) or DS1 (0.3 mg/kg) $5 \mathrm{~min}, 30 \mathrm{~min}$ or $45 \mathrm{~min}$ prior to an injection of cocaine $(20 \mathrm{mg} / \mathrm{kg})$ and locomotion was monitored for $60 \mathrm{~min}$. a) Data are expressed as mean total activity (+SEM). ${ }^{*} p<0.05$, data compared with Vehicle/Cocaine treated group, One-way ANOVA followed by Tukey test. b) Data expressed as mean ( \pm SEM) for locomotor activity measured at 5 min intervals. ${ }^{*} p<0.05$, for Vehicle/Cocaine vs. DS1/Cocaine (45 min) treated groups. Repeated measures Two-way ANOVA followed by Bonferroni post hoc test. $n=4-6$ for each group. 


\subsubsection{Effect of DS1 on cocaine prime induced reinstatement.}

The effect of various dose of DS1 (0, 0.1, 0.3 and $1.0 \mathrm{mg} / \mathrm{kg}$, ip) on cocaine (20 $\mathrm{mg} / \mathrm{kg}$, ip) induced drug-seeking is shown in Fig 2.6. Statistical analysis showed that pre-treatment with DS1 $(0.3,1.0 \mathrm{mg} / \mathrm{kg})$ attenuated cocaine prime induced reinstatement in a dose dependent manner $[\mathrm{F}(3,26)=36.82, \mathrm{p}<0.0001$; Fig 2.6a]. However, a trend towards increased active lever press responses was noted in animals treated with low dose DS1 $(0.1 \mathrm{mg} / \mathrm{kg})$, which was non-significant. The KOPr antagonist pre-treatment significantly reversed DS1 induced attenuation of cocaine reinstatement ( $\mathrm{p}<0.001$; Fig. 2.6b). 
(a)

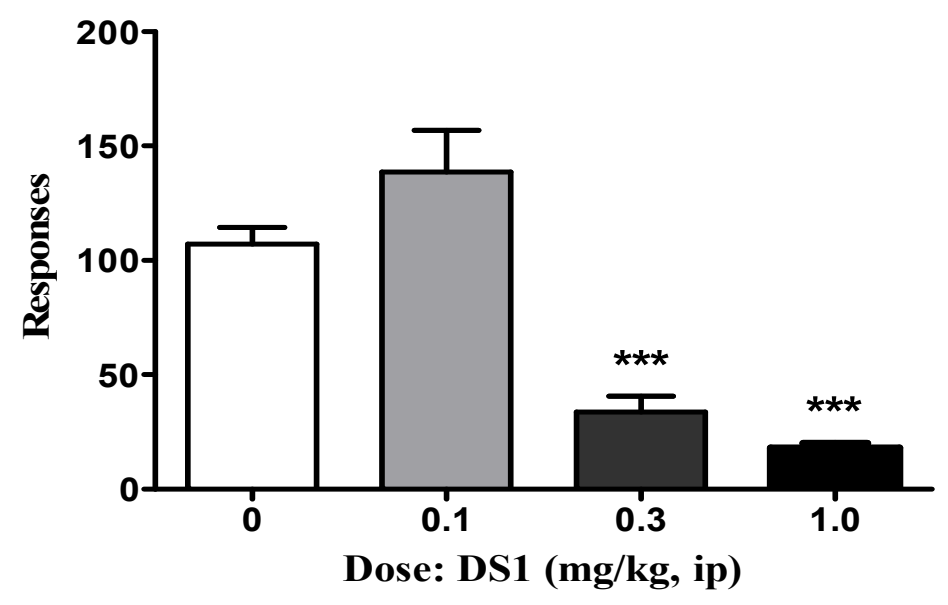

(b)

DS1 (0.3 mg/kg, ip)

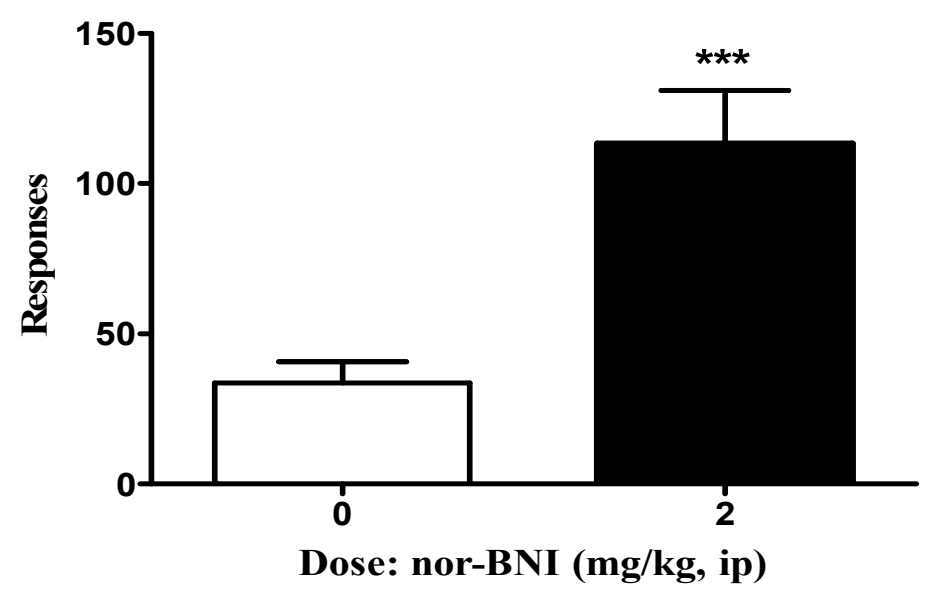

\section{Fig. 2.6. Effect of DS1 on cocaine prime induced reinstatement.}

Bars indicate active lever press responses + SEM produced during phase 3 of the reinstatement test. (a) Rats which had shown extinguished responding during the extinction phase were initially treated with different doses of DS1 (0, 0.1, 0.3 and $1.0 \mathrm{mg} / \mathrm{kg})$ followed by a priming injection of cocaine $(20 \mathrm{mg} / \mathrm{kg})$ and saline reinforced responses were recorded for a period of $60 \mathrm{~min}$. ${ }^{* *} p<0.001$, data compared with vehicle treated group $(0 \mathrm{mg} / \mathrm{kg})$. One-way ANOVA followed by Tukey post hoc test. $n=6-10$ for each group. (b) For nor-BNI reversal experiments, animals were first treated with nor-BNI $(0,2.0 \mathrm{mg} / \mathrm{kg})$ followed by DS1 $(0.3 \mathrm{mg} / \mathrm{kg})$ and cocaine $(20 \mathrm{mg} / \mathrm{kg})$ injections. ${ }^{* * *} p<0.001$, Student $t$-tests. $n=6$-10 for each group. 


\subsubsection{Effect of DS1 on cocaine induced hyperactivity (drug naüve animals)}

The effect of acute exposure to DS1 $(0,0.3 \mathrm{mg} / \mathrm{kg})$ on cocaine $(0,20 \mathrm{mg} / \mathrm{kg})$ induced hyperactivity is shown in Fig 2.7. A significant increase in the locomotion activity was observed in animals pre-treated with vehicle/cocaine as compared to those which were treated with vehicle/saline $(p<0.001)$ indicating cocaine induced hyperactivity in rats $[\mathrm{F}(3,26)=14.68, \mathrm{P}<0.0001$; Fig. $2.7 \mathrm{a}]$. Post hoc analysis indicated no significant difference between DS1 and vehicle pre-treatment on the locomotor activity produced following saline treatment $(\mathrm{P}>0.05$; Fig $2.7 \mathrm{a})$. However, an increase in total activity was observed in rats pre-treated with vehicle followed by an injection of cocaine $(20 \mathrm{mg} / \mathrm{kg})$ which was significantly attenuated by DS1 pre-treatment $(\mathrm{p}<0.01$; Fig 2.9a). Also, a further time course comparison between DS1 and vehicle treated groups showed that DS1 pre-treatment significantly suppressed locomotion 10, 20, 25, 30, 35 and $40 \mathrm{~min}(\mathrm{p}<0.05)$ following cocaine injection vs. vehicle treated group [treatment effect: $\mathrm{F}(3,276)=88.3, \mathrm{P}<0.0001$; interaction effect: $\mathrm{F}(33,276)=2.2, \mathrm{P}=$ 0.0003; Fig. 2.7b). 
(a)

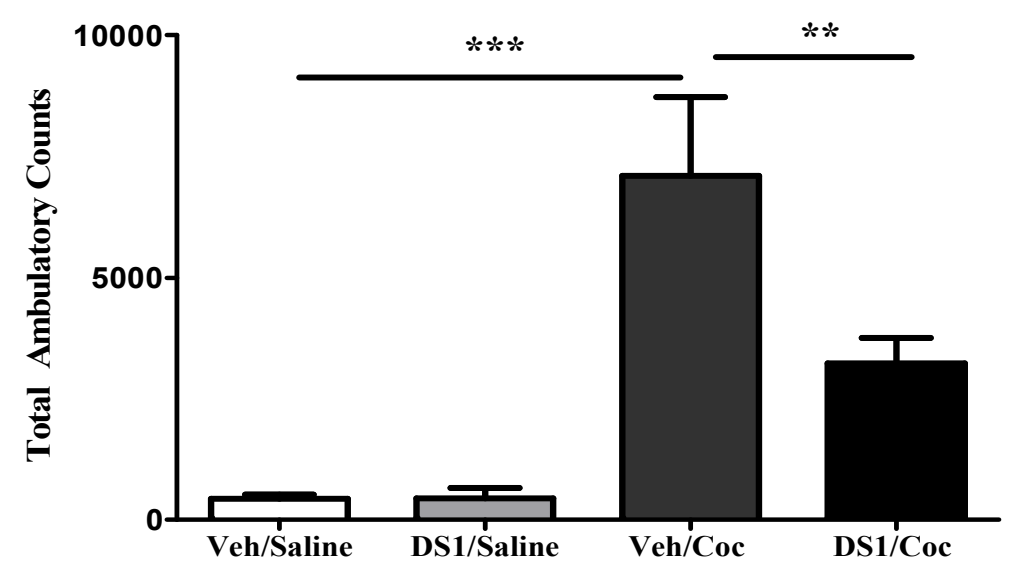

(b)

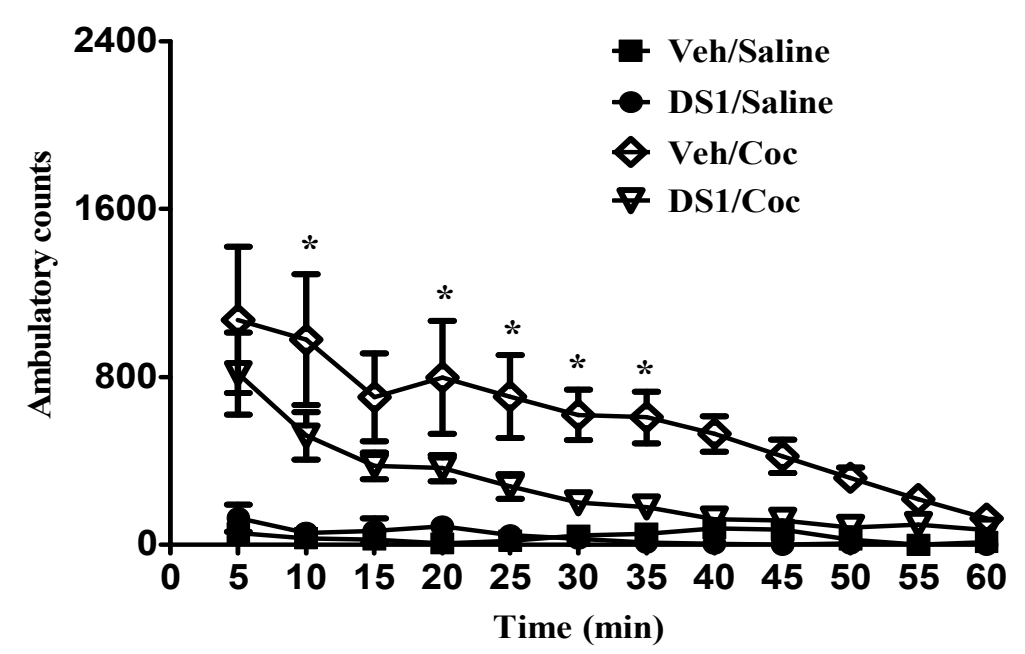

Fig. 2.7. Effect of DS1 on cocaine induced hyperactivity (drug naïve rats).

Drug naive rats were initially injected with either vehicle (Veh; 75\% DMSO) or DS1 (0.3 mg/kg) followed by saline (1 mL/kg) or cocaine (Coc; $20 \mathrm{mg} / \mathrm{kg}$ ) and total locomotion activity was monitored for 60 min. a) Figures represents mean total activity $+S E M . * * * p<0.001$, Veh/Saline vs. Veh/Coc treated group, ${ }^{* *} p<0.01$, Veh/Coc vs. DS1/Coc treated group. One-way ANOVA followed by Tukey post hoc test. b) Data expressed as mean \pm SEM for locomotor activity measured at 5 min intervals. ${ }^{*} p<0.05$, for Veh/Coc vs. DS1/Coc treated groups. Repeated measures Two-way ANOVA followed by Bonferroni post hoc test. $n=6-8$ for each group. 


\subsubsection{Effect of DS1 on expression of cocaine sensitization.}

The effect of DS1 $(0,0.3 \mathrm{mg} / \mathrm{kg}$, ip) exposure on the expression of cocaine sensitization in rats is shown in Fig 2.8. Animals that were pre-treated with cocaine on days 1-5 and exposed to vehicle followed by a cocaine injection on day 10 , showed a significant increase in locomotion compared to the group of animals which received saline from day $1-5$, indicating the expression of cocaine sensitization $[\mathrm{F}(3,26)=6.2$, $\mathrm{P}=0.0031$; Fig 2.8a]. Post-hoc tests showed no measurable difference in activity between animals exposed to DS1 on the test day after recieving either saline or cocaine on days 1-5 ( $\mathrm{P}>0.05$; Fig. 2.8a). However, a significant reduction in cocaine induced hyperactivity was observed in DS1 pre-treated animals vs. vehicle treated animals, all of which received cocaine on days 1-5 ( $<<0.05$; Fig 2.8a). Analysis of time course data also showed a significant treatment $[\mathrm{F}(3,276)=25.39, \mathrm{P}<0.0001]$ and interaction $[\mathrm{F}$ $(33,276)=3.84, \mathrm{P}<0.0001]$ effect (Fig. 2.8b). Post hoc tests showed a significant reduction in locomotion activity in DS1 pre-treated rats vs. the vehicle treated group at $5,10,15$ and 20 min following a single cocaine injection $(\mathrm{P}<0.05)$. Statistical analysis for cocaine induced stereotypy showed significant treatment effect $[\mathrm{F}(3,276)=3.6, \mathrm{P}<$ $0.05]$ but no interaction effect $[\mathrm{F}(33,276)=1.37, \mathrm{P}=0.24]$. Post-hoc tests showed no significant effect on cocaine induced stereotypic counts following DS1 pre-treatment vs. vehicle pre-treatment in cocaine sensitized animals ( $\mathrm{P}>0.05$; Fig 2.8c). However, DS1 pre-treatment showed a trend towards an increase in cocaine produced stereotypic counts in sensitized animals 5, 10 and 15 min post cocaine treatment (Fig 2.8c). 
(a)

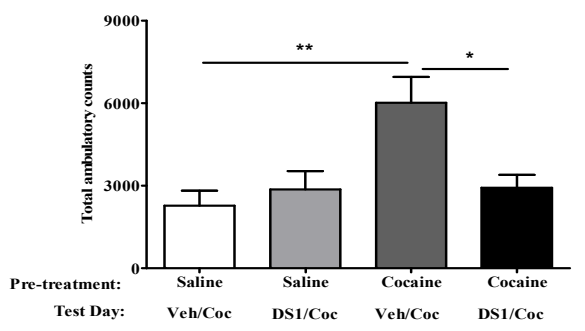

(b)
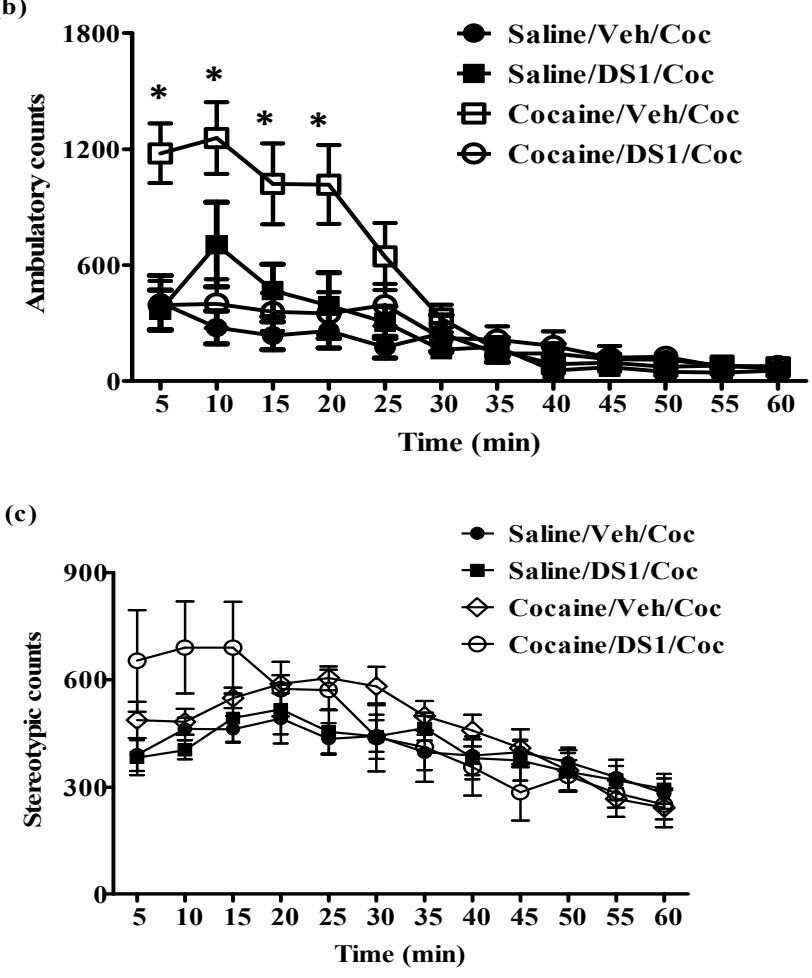

\section{Fig. 2.8. Effect of DS1 on expression of cocaine sensitization.}

Rats were injected with saline $(1 \mathrm{~mL} / \mathrm{kg})$ or cocaine $(20 \mathrm{mg} / \mathrm{kg})$ for 5 consecutive days. Animals remained drug free from day 6-9. On day 10, rats were injected with either vehicle (Veh, 75\% DMSO) or DS1 (0.3 mg/kg) and 45 min later were injected with cocaine (Coc, $20 \mathrm{mg} / \mathrm{kg}$ ) and activity was measured for $60 \mathrm{~min}$. a) Data are expressed as mean total activity +SEM. $*^{*}<0.01$ Saline/Veh/Coc vs. Cocaine/Veh/Coc treated group, $*_{p}<0.05$ Cocaine/Veh/Coc vs. Cocaine/DS1/Coc treated group. One-way ANOVA followed by Tukey post hoc test. b) Time course measurement of mean $\pm S E M$ of locomotion activity over 5 min intervals. ${ }^{*} p<0.05$ Cocaine/Veh/Coc vs. Cocaine/DS1/Coc treated group. Two-way ANOVA followed by Bonferroni post hoc test. $n=6-8$ for each group. (c) Time course measurement of mean ( \pm SEM) stereotypic counts for a period of $60 \mathrm{~min}$. Repeated measures two-way ANOVA followed by Bonferroni post hoc test. $n=6-8$ for each group. 


\subsubsection{Effect of MOM Sal B and EOM Sal B on cocaine prime induced reinstatement}

Effect of MOM Sal B and EOM Sal B on cocaine prime induced drug-seeking is shown in Fig 2.9. Statistical analysis showed that MOM Sal B $(0.3 \mathrm{mg} / \mathrm{kg})$ significantly attenuated cocaine prime induced reinstatement $[\mathrm{F}(3,17)=4.71, \mathrm{p}<0.05$; Fig 2.9a]. However, no significant decrease in cocaine prime induced lever press responding was noted following EOM Sal B exposure $[\mathrm{F}(3,17)=2.427, \mathrm{p}=0.989$; Fig 2.9b]. A nonsignificant trend towards increased active lever press responding was noted with MOM Sal B $(0.03,0.1 \mathrm{mg} / \mathrm{kg})$ and EOM Sal B $(0.1,0.3 \mathrm{mg} / \mathrm{kg})$. 
(a)

\section{Cocaine (20 mg/kg, ip)}

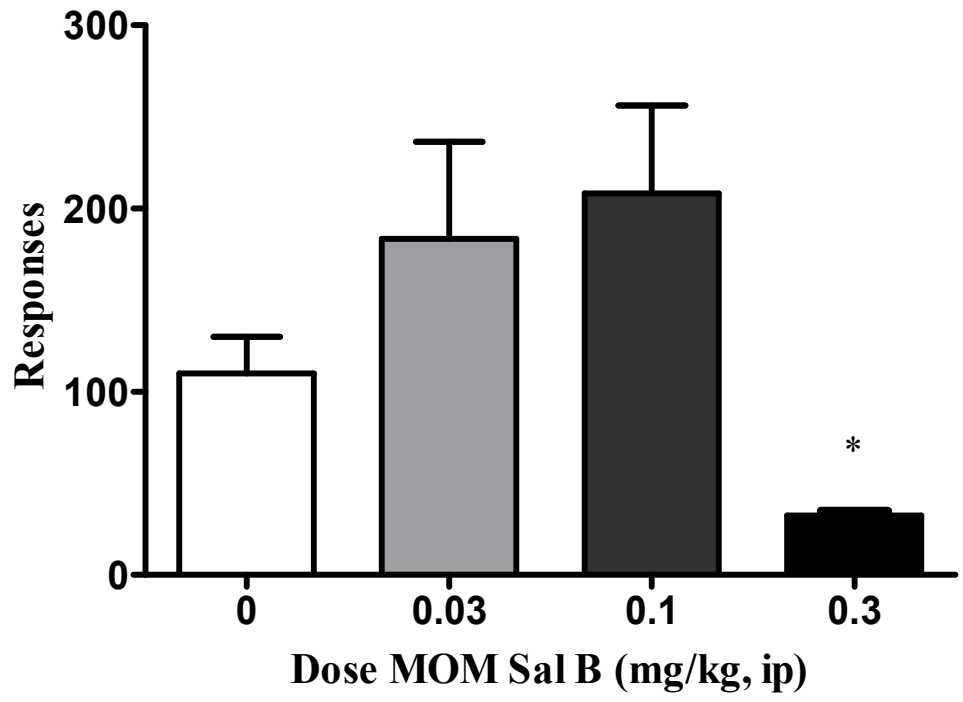

(b)

\section{Cocaine (20 mg/kg, ip)}

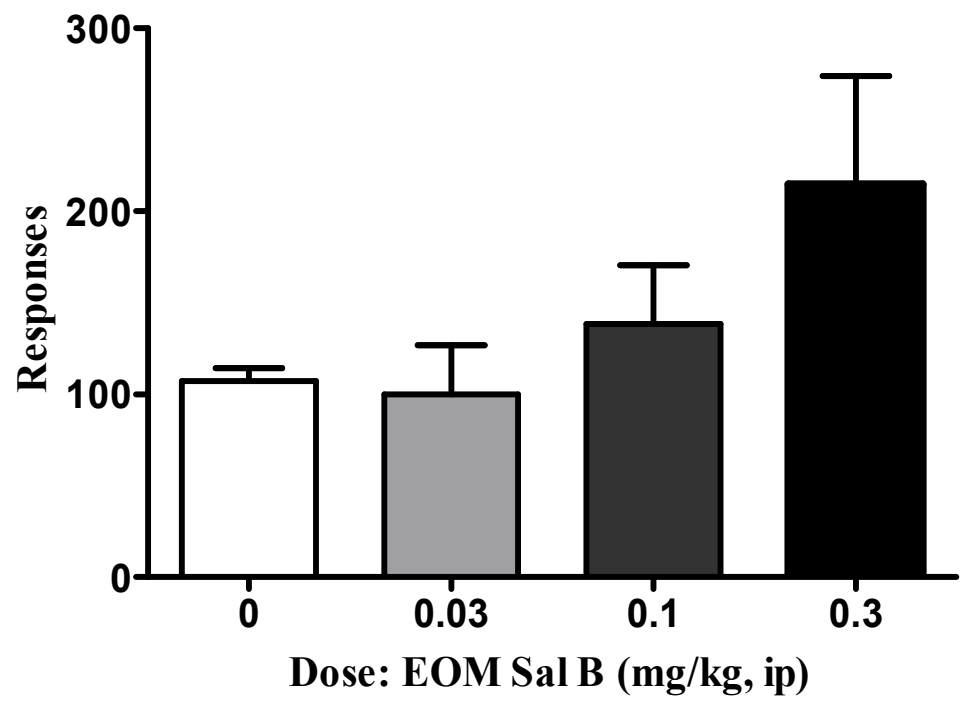

Fig.2.9. Effect of MOM Sal B and EOM Sal B on cocaine prime induced

\section{reinstatement.}

Symbols indicate active lever press responses +SEM produced during phase 3 of the reinstatement test. Rats which had shown extinguished saline reinforcements during the extinction phase were initially treated with different doses of (a) MOM Sal B (0, 0.03, 0.1 and $0.3 \mathrm{mg} / \mathrm{kg}$ ) and (b) EOM Sal B (0, 0.03, 0.1 and $0.3 \mathrm{mg} / \mathrm{kg})$ followed by a priming injection of cocaine $(20 \mathrm{mg} / \mathrm{kg})$ and saline reinforced responses were recorded for a period of $60 \mathrm{~min}$. ${ }^{*} p<0.05$, data compared with vehicle treated group $(0 \mathrm{mg} / \mathrm{kg})$. One-way ANOVA followed by Tukey post hoc test. $n=5-7$ for each group. 


\subsubsection{Effect of herkinorin on cocaine prime induced reinstatement.}

The effect of herkinorin pre-treatment on cocaine prime induced reinstatement is shown in Fig. 2.10. No significant change in responding was observed in rats pretreated with a high dose of herkinorin $[0.3,1.0 \mathrm{mg} / \mathrm{kg} ; \mathrm{F}(3,24)=1.48, \mathrm{p}=0.245]$. However, a non-significant $(\mathrm{p}>0.05)$ trend towards an increase in lever press responding was observed in animals treated with a low dose of herkinorin $(0.1 \mathrm{mg} / \mathrm{kg})$. Also, a trend in decreasing responses was noted in rats treated with the higher dose of herkinorin $(0.3,1.0 \mathrm{mg} / \mathrm{kg})$, which was non-significant. 


\section{Cocaine (20 mg/kg, ip)}

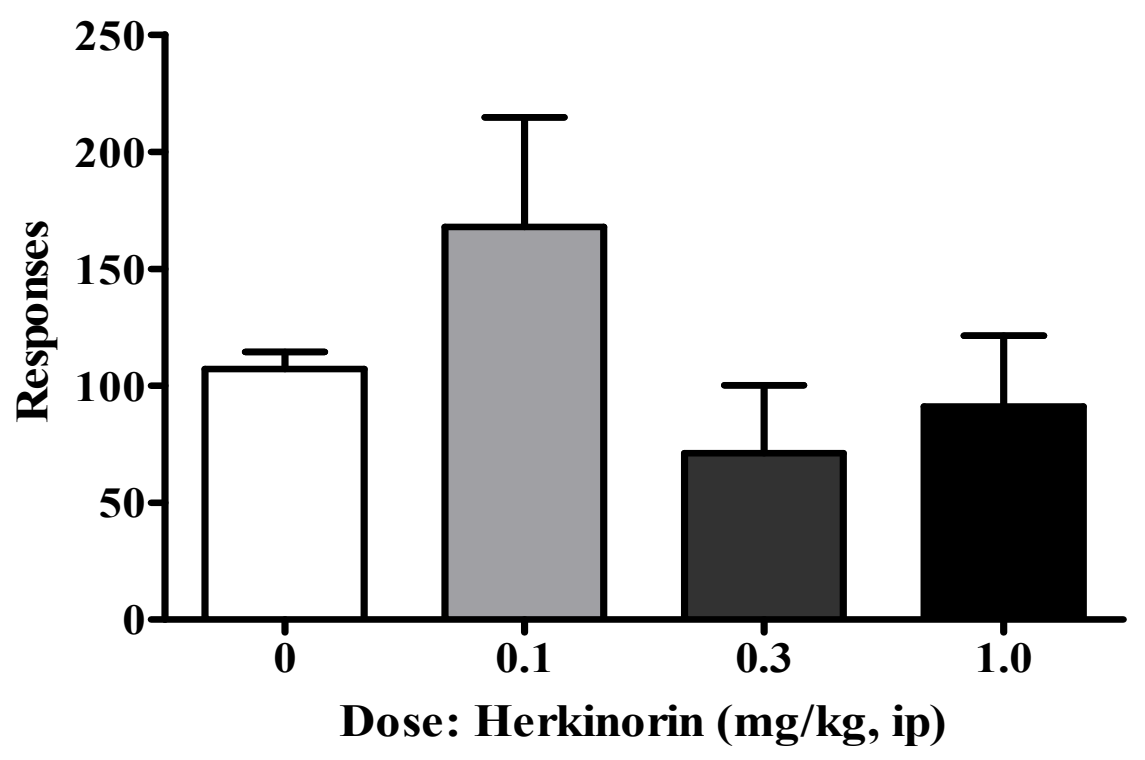

\section{Fig.2.10. Effect of herkinorin on cocaine prime induced reinstatement.}

Symbols indicate active lever press responses +SEM produced during phase 3 of the reinstatement test. Rats which had shown extinguished saline reinforcements during the extinction phase were initially treated with different doses of herkinorin $(0,0.1,0.3$ and 1.0 $\mathrm{mg} / \mathrm{kg}$ ) followed by a priming injection of cocaine $(20 \mathrm{mg} / \mathrm{kg})$ and saline reinforced responses were recorded for a period of 60 min. One-way ANOVA followed by Tukey post hoc test. $n=5$ 10 for each group. 


\subsubsection{Effect of nalmefene on cocaine prime induced reinstatement.}

The effect of a single injection of nalmefene $(0,0.1,0.3$ and $1.0 \mathrm{mg} / \mathrm{kg})$ on cocaine prime-induced reinstatement is shown in Fig. 2.11. Statistical analysis showed a significant attenuation in cocaine prime-induced drug-seeking in nalmefene pre-treated groups when compared to the vehicle treated group of rats $[F(3,17)=6.48, \mathrm{p}<0.01]$. This effect produced by nalmefene $(0.3,1.0 \mathrm{mg} / \mathrm{kg})$ was reversed by nor-BNI $(0,2.0$ $\mathrm{mg} / \mathrm{kg}$ ) pre-treatment $(\mathrm{p}<0.001 ;$ Fig $2.11 \mathrm{~b})$. 
(a)

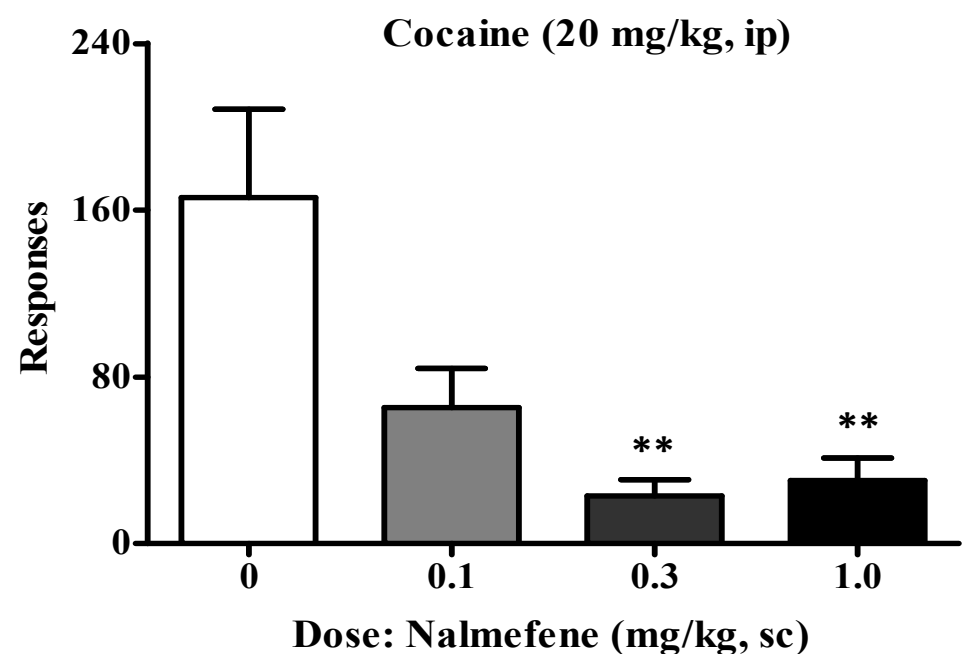

(b)

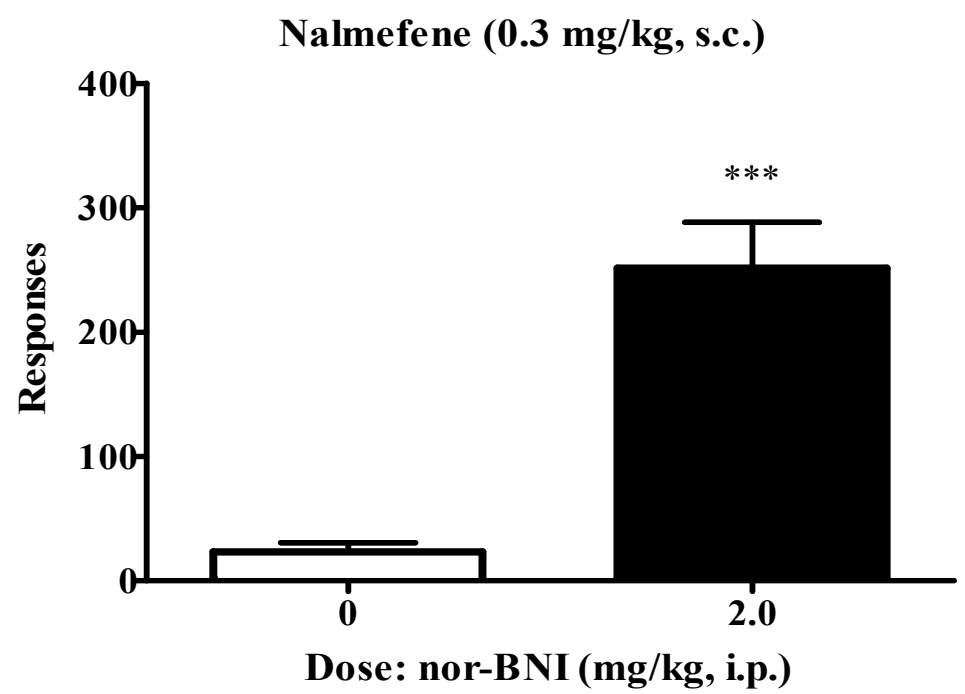

Fig. 2.11. Effect of nalmefene on cocaine prime induced reinstatement.

Symbols indicate active lever press responses +SEM produced during phase 3 of the reinstatement test. (a) Rats which had shown extinguished saline responding during the extinction phase were initially treated with different doses of nalmefene $(0,0.1,0.3$ and 1.0 $\mathrm{mg} / \mathrm{kg})$ followed by a priming injection of cocaine $(20 \mathrm{mg} / \mathrm{kg})$ and saline reinforced responses were recorded for a period of $60 \mathrm{~min} .{ }^{* *} p<0.01$, data compared with vehicle treated group $(0$ $m g / k g$ ). One-way ANOVA followed by Tukey post hoc test. $n=5-6$ for each group. (b) For nor$B N I$ reversal experiments, animals were first treated with nor-BNI $(0,2.0 \mathrm{mg} / \mathrm{kg})$ followed by nalmefene $(0.3 \mathrm{mg} / \mathrm{kg})$ and cocaine $(20 \mathrm{mg} / \mathrm{kg})$ treatment. ${ }^{* * *} p<0.001$, Student $t$-tests. $n=5-6$ for each group. 


\subsection{Summary of results}

Tab. 2.3. Effect of KOPr agonists on cocaine prime induced drug-seeking

\begin{tabular}{|c|c|c|}
\hline Compound tested & $\begin{array}{c}\text { Cocaine prime induced } \\
\text { reinstatement }\end{array}$ & $\begin{array}{c}\text { KOPr antagonist } \\
\text { induced reversal }\end{array}$ \\
\hline Sal A & Attenuated (selective) & Reversed \\
\hline DS1 & Attenuated (selective) & Reversed \\
\hline MOM Sal B & Attenuated (non-selective) & - \\
\hline EOM Sal B & No effect & - \\
\hline Herkinorin & No effect & - \\
\hline Nalmefene & Attenuated (selective) & Reversed \\
\hline
\end{tabular}

$\underline{\text { Tab 2.4. Effect of KOPr agonists on cocaine induced motor activity }}$

\begin{tabular}{|c|c|c|c|}
\hline Compound tested & $\begin{array}{c}\text { Cocaine induced } \\
\text { hyperactivity }\end{array}$ & \multicolumn{2}{|c|}{$\begin{array}{c}\text { Expression of cocaine } \\
\text { behavioural sensitization }\end{array}$} \\
\cline { 3 - 4 } & Potentiated & $\begin{array}{c}\text { Motor } \\
\text { sensitization }\end{array}$ & $\begin{array}{c}\text { Stereotypic } \\
\text { counts }\end{array}$ \\
\hline Sal A & Attenuated & No effect \\
\hline DS1 & Attenuated & Attenuated & No effect \\
\hline
\end{tabular}




\subsection{Discussion}

In the present study, the novel neoclerodane diterpene KOPr agonist Sal A and its structural analogues DS1, MOM Sal B, EOM Sal B, herkinorin and partial KOPr agonist/MOPr antagonist, nalmefene, were tested for their anti-addiction behaviours using cocaine prime induced reinstatement in rats. To determine if their behavioural effects were mediated via KOPr activation, self-administration animals were also pretreated with the selective KOPr antagonist nor-BNI. The anti-cocaine reinstatement effects produced by novel KOPr agonists that attenuated cocaine seeking behaviours selectively without producing sedation by themselves (Chapter 3) were subjected to further behavioural tests. These tests included the measurement of cocaine induced behavioural sensitization and stereotypic counts. Effect of acute cocaine induced hyperactivity was also measured in rats.

Previous studies have shown that a single systemic injection of cocaine (20 $\mathrm{mg} / \mathrm{kg}$, ip) reinstates extinguished cocaine reinforced responding behaviour (Worley et al., 1994; Schenk and Partridge, 1999a; Schenk et al., 2000a). Injecting $20 \mathrm{mg} / \mathrm{kg}$ cocaine for 5 consecutive days has been shown to produce a sensitized response to cocaine. This has been shown by a decreased latency in self-administration acquisition (Schenk and Partridge, 2000b). Furthermore, cocaine (20 mg/kg, ip) when injected for 5 consecutive days also produces sensitization to cocaine's motor stimulating effects (Heidbreder et al., 1995; 1996). The results from this study support these previous findings as shown by cocaine $(20 \mathrm{mg} / \mathrm{kg}$, ip) initiated prime induced reinstatement (Fig 2.1) and expression of motor sensitization in rats (Fig 2.4; Fig 2.8).

Previous studies have shown that conditioning stimuli can play an important role in the expression of motor sensitized responses in laboratory animals (Post et al., 1981; Beninger and Herz, 1986). In the present study, rats which received cocaine or saline injections for 5 consecutive days were returned to their home cage after drug/vehicle 
exposures. Therefore, the difference in ambulation which was measured in the locomotion chamber after cocaine was administered on the test day (day 10) was specifically due to cocaine exposures during pre-treatment days 1-5 (Fig 2.3; Fig 2.8). The presence of the house light above the active lever has been considered to be conditioned stimuli and has been used for training animals to self-administer cocaine (Schenk and Partridge, 2001a). Therefore, a light stimulus was presented just above the active lever and was lit whenever the animal pressed the active lever during this cocaine self-administration study. Consistent with this, presentation of such drug associated cues have been shown to initiate the reinstatement of extinguished drug-seeking (Stewart et al., 1984). In order to avoid any cue-induced effects while testing during the reinstatement phase, the light stimulus was presented to the animals above the active lever throughout all the phases of the reinstatement test. Thus, increase in the lever press responses observed during the self-administration phase followed by a decrease in lever press responding during the extinction phase may be attributed only to the presence of cocaine and saline in the infusion pump during phase 1 and 2 respectively (Fig. 2.1).

No previous in vivo work has been reported on the Sal A analogue, DS1. Therefore the onset of action time for DS1 $(0.3 \mathrm{mg} / \mathrm{kg})$ on cocaine induced hyperactivity was performed at various pre-treatment times. DS1 showed a significant suppression in hyperactivity produced when injected $45 \mathrm{~min}$, but not 5 or $30 \mathrm{~min}$ prior to receiving cocaine injections (Fig. 2.5). This finding suggests that DS1 has a slower, more prolonged onset of action in vivo compared to Sal A (2-5 min; Schmidt et al., 2005). Behavioural tests measuring KOPr agonist induced analgesia have been previously used to determine the duration of action of the compound tested (Wang et al., 2008; John et al., 2005). Such tests apply the hot plate, tail flick and acetic acid induced writhing assays in laboratory animals (John et al., 2005; McCurdy et al., 2005; Wang et 
al., 2008). As the duration of action of DS1 still remains unanswered, detailed studies using these behavioural paradigms are therefore warranted.

Results from this study indicate that Sal A and DS1 both attenuated cocaine prime induced drug-seeking behaviour (0.3, $1.0 \mathrm{mg} / \mathrm{kg}$; Fig 2.2a; Morani et al., 2009; Fig 2.6a; Tab 2.3). In order to further characterise their anti-addiction potential, the effect of acute exposure to Sal A and DS1 $(0.3 \mathrm{mg} / \mathrm{kg})$ was tested on cocaine induced stimulation of motor function. Sal A and DS1, at the minimum effective dose which attenuated drug-seeking behaviour $(0.3 \mathrm{mg} / \mathrm{kg})$, significantly suppressed the expression of cocaine behavioural sensitization in rats (Fig 2.4; 2.8; Tab 2.4). In addition to its effect on cocaine sensitization, DS1 $(0.3 \mathrm{mg} / \mathrm{kg})$ pre-treatment significantly suppressed cocaine induced hyperactivity (Fig 2.7; Tab 2.4). In contrast, Sal A (0.3 mg/kg) exposure significantly increased motor function produced by a single cocaine injection (Fig. 2.3; Tab 2.4). Contrary to this present finding, a recent report by Chartoff and colleagues (2008) suggested that Sal A $(2.0 \mathrm{mg} / \mathrm{kg})$ pre-treatment decreases cocaine induced hyperactivity in rats (Chartoff et al., 2008). One of the reasons for the differences observed might be due to the relatively low dose of Sal A $(0.3 \mathrm{mg} / \mathrm{kg}$ vs. 2.0 $\mathrm{mg} / \mathrm{kg}$; Chartoff et al., 2008) and relatively high dose of cocaine (20 mg/kg vs. 10 $\mathrm{mg} / \mathrm{kg}$; Chartoff et al., 2008) used in this study. In contrast to its effect on behavioural sensitization, Sal A and DS1 pre-treatment produced opposite effects on acute cocaine induced activity. Sal A significantly enhanced whereas DS1 significantly suppressed cocaine motor function. This observed discrepancy might reflect the difference in the pharmacokinetic properties of these compounds as Sal A has a quick onset of action (5 min; Schmidt et al., 2005b) compared to DS1 (45 min; Fig 2.5).

An increase in total activity was noted in animals treated with cocaine on the test day following no- (Fig 2.3, 2.5, 2.7) or prior-cocaine exposures (Fig 2.4, 2.8) when compared to saline injections. Thus indicating the presence of cocaine induced 
hyperactivity and expression of cocaine sensitization. On close observation, a greater enhancement in motor function was noted in animals receiving vehicle followed by acute cocaine challenge (Fig 2.3, 2.7) compared to animals pre-treated with saline for 5 days followed by cocaine treatment on test day Fig 2.4, 2.8). Additionally, the sensitized animals (i.e. those receiving 5 days of cocaine pre-treatment; Fig 2.4, 2.8) had a cocaine response that was similar to that observed in naïve animals treated with acute cocaine (Fig 2.3, 2.7). A possible explanation for these discrepancies may include batch differences as both of these experiments were performed at different times.

An increase in stereotypy behaviour is generally not favoured by forward locomotion (Post et al., 1987). Repeated cocaine treatment or high dose of cocaine (20 $\mathrm{mg} / \mathrm{kg}$ ) has been shown to initially enhance locomotion activity followed by an increase in stereotypy behaviours in laboratory rats (Post et al., 1987; Ushijima et al., 1995). Therefore, we analyzed the number of stereotypic counts in cocaine sensitized animals treated with either Sal A or DS1. There was no significant effect on cocaine produced stereotypy with Sal A or DS1 pre-treatment (Fig 2.4; 2.8). However, a non-significant enhancement in cocaine produced stereotypy was noted in both Sal A and DS1 treated animals which coincided with the suppression of motor sensitization (5-15 min post cocaine treatment; Fig 2.4, 2.8). Thus, the decrease in cocaine-induced behavioural sensitization produced by both the KOPr compounds might reflect either a decrease in the ability of cocaine to produce horizontal activity or an increase in the ability of cocaine to produce the competing behaviour of stereotypy following the administration of higher doses of psychostimulants (Ushijima et al., 1995; Post et al., 1987). Further studies are warranted to define the effects of Sal A and DS1 on cocaine induced stereotypic behaviours. Such experiments have been described in details in Chapter 4 .

Ultrastructural studies by Svingos and colleagues (2001) have shown that KOPr and DAT are co-localized in the NAc and therefore activation of KOPr can modulate 
cocaine induced behaviours via DAT. Because of the interaction between KOPr and dopaminergic mechanisms, a focus on the effects of Sal A on dopamine-mediated behaviours has been of interest (Gherke et al., 2008; Chartoff et al., 2008). In drug discrimination tests, Sal A and its synthetic derivatives, MOM Sal B and EOM Sal B, substituted completely for U69593 (Baker et al., 2009), suggesting they share effects mediated via the KOPr. Cocaine-seeking has been attributed to dopaminergic mechanisms (Schmidt et al., 2005a; Section 1.4; Chapter 1), which is supported by data showing that Sal A modulates the effects of cocaine through interactions with D1 receptor mediated signalling pathways in the dorsal striatum (Chartoff et al., 2008). Also, Sal A pre-treatment has been shown to decrease DA levels in the NAc (Carlezon et al., 2006) and CPU (Zhang et al., 2005; Gherke et al., 2008). However, the effects produced by Sal A might be bi-phasic as a lower dose of Sal A $(40 \mu \mathrm{g} / \mathrm{kg})$ increased DA levels in NAc (Braida et al., 2008) whereas higher doses $(1.0-3.2 \mathrm{mg} / \mathrm{kg})$ decreased DA levels in dorsal (Zhang et. al., 2005) and ventral (Carlezon et al., 2006) striatum. Therefore, the ability of moderate to high doses of Sal A to decrease cocaine-produced drug-seeking might be due to its effect on the dopaminergic system. However, further work is required to link the role of Sal A in modulating DA neurotransmission with its attenuation of cocaine seeking behaviour.

Sal A analogues, MOM Sal B (Wang et al., 2008) and EOM Sal B (Hooker et al., 2009), have a longer duration of action than Sal A. These compounds were tested for their effects on cocaine prime-induced drug-seeking behaviour. EOM Sal B, which is 10 times more potent than Sal A at KOPr (Beguin et al., 2005; Munro et al., 2008), had no effect on cocaine prime induced drug-seeking behaviour. However, a trend in enhancement of active lever press responding was observed when rats were pre-treated with the higher doses of EOM Sal B $(0.1,0.3 \mathrm{mg} / \mathrm{kg}$; Fig 2.9b; Tab 2.3). On the other hand, Sal A at the same dose $(0.3 \mathrm{mg} / \mathrm{kg})$ significantly attenuated cocaine-seeking (Fig 
2.2; Tab 2.3). A recent study by Hooker et al., (2009) showed that EOM Sal B was metabolically more stable than Sal A. Additionally, EOM Sal B when injected ip showed a gradual decline in the whole brain concentrations in the rats (Hooker et al., 2009). Also, EOM Sal B showed less affinity for plasma proteins in baboons reflecting more bioavailability (Hooker et al., 2009). These data are suggestive that differences between EOM Sal B and Sal A with respect to cocaine seeking might reflect their different pharmacokinetic profiles. MOM Sal B, is approximately 7 times more potent than Sal A at KOPr (Beguin et al., 2005; Munro et al., 2008), at low doses (0.03, 0.1 $\mathrm{mg} / \mathrm{kg}$ ) MOM Sal B also showed a non-significant trend towards an increase in active lever press responding. However at a higher dose $(0.3 \mathrm{mg} / \mathrm{kg}), \mathrm{MOM}$ Sal B significantly attenuated drug-seeking behaviour (Fig 2.9a; Tab 2.3). It was interesting to observe that although EOM Sal B and MOM Sal B are both potent and long acting Sal Aanalogues, they produce dissimilar effects on cocaine-seeking behaviour at the high dose tested (0.3 mg/kg; Fig 2.9). Further studies are required to explain these dissimilarities. As, KOPr activation has been shown to decrease natural reward seeking behaviour (Mello and Negus, 2000), it would be interesting to observe the effect of MOM Sal B (0.3 $\mathrm{mg} / \mathrm{kg}$ ) pre-treatment in animals trained to obtain natural reward related reinforcements, like sucrose. This would help in determining the degree of selectivity of MOM Sal B in attenuation of cocaine-seeking behaviour. The results from such tests have been discussed in the following chapter (Chapter 3).

Herkinorin has a higher affinity for MOPr than KOPr (Harding et al., 2005; Tidgewell et al., 2006; Prisinzano and Rothman, 2008), and was tested for its capacity to reinstate cocaine induced behaviour. Herkinorin pre-treatment had no significant effect on cocaine prime induced drug-seeking (Fig 2.10; Tab 2.3). However, it was interesting to observe that there was a slight trend towards a decrease in lever press responding in rats pre-treated with high doses $(0.3,1.0 \mathrm{mg} / \mathrm{kg})$ of herkinorin. 
MOPr agonists such as methadone and its derivatives have been used clinically for opioid maintenance therapy (Paulus and Halliday, 1967; Section 1.1, Chapter 1). However, one of the problems associated with methadone is that it is itself addictive and in some cases might also lead to fatal overdosing (Reingardiene et al., 2009). It is well known that KOPr activation opposes MOPr activation related behaviours (Pan, 1998; Section 1.7; Chapter 1). Therefore, developing Sal A analogues which activates MOPr as well as KOPr may have the potential to be developed into pharmacotherapies to manage opioid withdrawal effects. A recent study by Groer and colleagues, (2007) also showed that herkinorin, unlike other MOPr agonists, did not promote beta-arrestin recruitment to MOPr or cause receptor internalization. Further detailed behavioural and neurochemical studies with herkinorin are required to explore its usefulness in drug withdrawal. Future studies with herkinorin as maintenance therapy has been discussed in Chapter 4. Since herkinorin is the only Sal A analogue with MOPr affinity, a sense of interest has been generated in developing herkinorin analogues possessing analgesic effects with fewer adverse effects compared to selective MOPr agonists (Harding et al., 2005; Tidgewell et al., 2006; 2008; Prisinzano and Rothman, 2008).

KOPr ligands having affinities for other opioid receptors have previously been suggested to attenuate cocaine induced behaviours with safer adverse effect profile than selective KOPr agonists (Archer et al., 1996; Bowen et al., 2003; Stevenson et al., 2004; Kreek et al., 2005; Bart et al., 2005). Additionally, bremazocine and cyclazocine (KOPr agonists with MOPr antagonist actions) have been shown to modulate cocaine induced behaviours in laboratory animals (Archer et al., 1996; Vanderschuren et al., 2000; Glick et al., 1998; Section 1.7; Chapter 1). The present study showed that nalmefene, which is a MOPr antagonist having partial agonist activity at KOPr attenuated cocaine prime induced drug-seeking behaviour $(0.3,1.0 \mathrm{mg} / \mathrm{kg}$; Fig $2.11 \mathrm{a})$. This study is the first to present pre-clinical evidence suggesting that nalmefene attenuates cocaine induced 
behaviour (Tab 2.3). Ligands activating KOPr and antagonising MOPr functions have been proposed as anti-addiction agents with possibly less adverse effects as compared to selective KOPr agonists (Mello and Negus, 2000; Bowen et al., 2003; Wang et al., 2009). Since nalmefene has been shown to attenuate cocaine induced drug-seeking in rats, it would be interesting to note the extent to which a single exposure to nalmefene produces KOPr related adverse effects. Further work in this regard was undertaken and is described in the following chapter (Chapter 3).

In order to determine if KOPr activation by Sal A, DS1 and nalmefene was in fact due to selective KOPr activation cocaine-seeking behaviour in the presence of KOPr antagonist nor-BNI was also determined. Animals pretreated with Nor-BNI 30 min before injection of KOPr agonists (Sal A, DS1 and nalmefene) completely reversed the attenuation in drug-seeking behaviour, thus confirming a KOPr mediated effect (Fig. 2.2b; 2.6b; 2.11b; Tab 2.3). Nalmefene is also an antagonist at MOPr (Bart et al., 2005; Ingman et al., 2005), therefore, the role of MOPr antagonism in the nalmefene induced anti-cocaine effects needs to be further characterised. KOPr activation has been shown to reduce extracellular DA levels in the NAc, an effect reversed with nor-BNI pretreatment (Spanagel et al., 1990). Thus, a reduction in extracellular DA levels in the NAc by KOPr activating compounds might reflect its ability to attenuate cocaineseeking behaviour. To validate this hypothesis future studies are required and have been discussed in Chapter 4.

It was interesting to note an enhancement in active lever-pressing in animals pretreated with nor-BNI $(181.8 \pm 39.38$, Fig $2.2 \mathrm{~b} ; 251.2 \pm 37.21$, Fig 2.11b) compared to control (0 mg/kg) animals (107.1 \pm 7.3, Fig 2.2a; $166.2 \pm 42.39$, Fig 2.11a). Similarly, Beardsley and colleagues (2005) also reported a non-significant potentiation of cocaine prime induced reinstatement in rats pre-treated with JDTic, a selective KOPr antagonist. Thus, enhancement in the cocaine's reinforcing effects by KOPr antagonists might be 
via the blockade of endogenous KOPr tone. This also shows that opposite effects are produced by KOPr antagonists' vs KOPr agonists on cocaine seeking (Fig 2.2, 2.11).

As explained in Section 2.1, the cocaine induced reinstatement paradigm has been extensively used to determine the effectiveness of pharmacological agents in producing anti-addiction effects. The within-session reinstatement paradigm however has its limitations. This model does not take into account the neuroadaptations associated with long term withdrawal from the drug self-administration. Such neuroadaptations are considered critical for the emergence of negative reinforcement and for expression of craving and relapse (Self, 2004; Self et al., 2004; Wolf, 2010; Koob \& Volkow, 2010; Feltenstein \& See, 2008). Therefore, the effect of modulating KOPr system on long term withdrawal from cocaine self-administration needs further assessment and can be performed using a between session reinstatement test. Such studies have the potential to determine the effect of $\mathrm{KOPr}$ activation on neuroadaptations associated with withdrawal and relate them to relapse.

The within-reinstatement model exhibits low predictive power by itself and results obtained using this paradigm fail to discriminate between false positives and compounds producing anti-addiction behaviour. This can be illustrated by considering the possible effects of dopamine agonists and antagonists on this model. The neuroleptic haloperidol or the D1 antagonist SCH23390, at doses that do not induce catalepsy or motor inhibition by themselves, may block cocaine-induced reinstatement of drug seeking. However, we might not think of them as potential or safe therapeutics for cocaine abuse. These probable results would, at least, imply the role of DA receptors in cocaine-induced reinstatement (Self, 2004). Additionally, it would be interesting to consider the effect of DA agonist on measuring cocaine-seeking behaviour in this model. One example is apomorphine, a classical mixed D1/D2 receptor agonist which, at low doses, produces sedative, yawning and motor inhibition 
effects (Ernst, 1967). If apomorphine attenuates cocaine-seeking at this dose, it might reflect a false positive effect. Furthermore, low doses of apomorphine via D2 autoreceptors have been shown to inhibit the synthesis and release of DA (Anderson and Gazzara, 1993), an effect similar to that elicited by KOPr activation (Shippenberg et al., 2007). Apomorphine suppresses cocaine-induced reinstatement of drug-seeking (De Vries et al., 1999), however, it is ineffective at treating cocaine addiction. Also the D3 receptor agonist' quinpirole and 7- OH-DPAT have been shown to produce similar inhibitory profiles in attenuating cocaine self-administration (Caine and Koob, 1993), but are unlikely to be used as an anti-addiction pharmacotherapies. Taken together, these data suggest that the within-session reinstatement model could be applied as a preliminary screening tool to identify the effectiveness of neoclerodane diterpenes as anti-addiction compounds. Further screening is therefore required to coin the effectiveness of KOPr compounds in modulating addictive behaviours and such experiments are described in detail in chapter 4 .

KOPr compounds that attenuate cocaine-seeking using the within-session reinstatement model will need further evaluation to discriminate them from false positives. To do so, we have applied control experiments which were aimed to measure motor suppression during reinstatement test and effect of KOPr compounds on natural reward related behaviour in rats. Data from these experiments have been described in the following chapter (Chapter 3).

\subsection{Summary and conclusions}

The results from this chapter indicate that Sal A and its analogues MOM Sal B, DS1 and partial KOPr agonist/ MOPr antagonist, nalmefene attenuated cocaine primeinduced drug seeking behaviour in rats in a similar way to traditional KOPr agonists. Sal A and DS1 also attenuated expression of cocaine behavioural sensitization, with 
DS1 also attenuating cocaine induced hyperactivity in rats. Thus given the anti-cocaine profile for these novel KOPr analogues, it will be interesting to note if they produce KOPr agonist related adverse effects such as anhedonia, depression, aversion and motor suppression. The next chapter deals with the adverse effect profiles of these novel KOPr agonists. 
Chapter.3. Side effect profile of novel KOPr agonists 


\subsection{Introduction}

\subsubsection{Adverse effects of traditional KOPr agonists}

Although, KOPr agonists attenuate cocaine induced behaviours, they are also associated with producing adverse effects such as conditioned place and taste aversion, depression, hallucination, sedation, emesis and decreased responding for food reinforcements in laboratory animals (Todtenkopff et al., 2004; Butelman et al., 2010; Mello and Negus, 2000; Section 1.5, Chapter 1). Also, clinically, KOPr activation has been shown to induce depersonalization, confusion, visual distortions and hallucinations (Pfeiffer et al., 1986; Walsh et al., 2001a; Johnson et al., 2011; Section 1.5, Chapter 1). These adverse effects associated with KOPr agonists have limited their development as anti-addiction therapeutics (Walsh et al., 2001a). Due to the undesirable effects associated with KOPr agonists, we performed behavioural adverse effect screening on the compounds which successfully attenuated cocaine-seeking behaviour (Chapter 2).

\subsubsection{Pre-clinical models to screen side effect profile of KOPr agonists}

The following section gives a brief account on the behavioural models used to screen the adverse effect profile for novel KOPr agonists. The spontaneous open field activity test has been used to determine the motor suppressant effects of traditional KOPr agonists (U50488H, U69593, spiradoline) pre-clinically (Sction 1.5, Chapter 1; Wadenberg, 2003; Lahti et al., 1982; 1985). The principle for sucrose reinforcement test and CTA has been described in Section 1.3, of Chapter 1. A recent study by Davis and colleagues (2009) has shown that a single pairing of U50488H with novel tasting saccharin solution produces taste aversion. Also, a previous study by Mucha and Herz, (1985) showed that traditional KOPr agonists (U50488H, bremazocine, ethylketazocine, trifluadom) produced taste and place aversion in a dose dependent manner. Therefore, in this present study, CTA was applied to measure the aversive behaviour produced by a single KOPr agonist exposure in rats. 
The FST (Porsolt et al., 1979) has been used extensively as an animal model of depressive like behaviours (Porsolt et al., 1979; Carlezon et al., 2006) and to screen pharmacological anti-depressants in laboratory animals (Detke et al., 1995). In the FST, the animals are habituated to force swimming in a cylindrical chamber for $15 \mathrm{~min}$. The next day, animals having been pharmacologically treated during the intervening period, are again placed in the cylindrical chamber and the frequency of these behaviours (climbing, swimming and immobility) is noted for $5 \mathrm{~min}$. If the treated animal spends more time immobile and relatively less time swimming or climbing, it then indicates possible depressive effects induced by the pharmacological agent (Porsolt et al., 1979; Carlezon et al., 2006). On the other hand, more time spent either climbing or swimming (active behaviours) and less time spent immobile, indicates potential anti-depressant like effect of the administered pharmacological agent (Detke et al., 1995).

\subsubsection{KOPr mediated SERT modulation}

SERT belongs to the sodium-chloride symporter protein family and plays a prominent role in 5HT neurotransmission by clearing 5HT from the synaptic cleft (Blakely et al., 1994; Saier, 1999; Zahniser and Doolen, 2001; Rudnick, 2006). Alteration of5HT neurotransmission has been implicated in numerous psychiatric disorders such as depression (Owens and Nemeroff, 1994), anxiety (Suranyi-Cadotte et al., 1990), bipolar disorder (Bellivier et al., 1997; Velayudhan et al., 1999) and addiction to psychostimulants (Heinz et al., 2001; Bauman and Rothman, 1998). It is well documented that KOPr's are co-localized with DAT in the NAc (Svignos et al., 2001) and KOPr agonists modulate DA neurotransmission by increasing DAT function in the NAc (Thompson et al., 2000). However, little is known about their effects on modulating the function of other monoamine transporters. Therefore, it would be interesting to understand whether KOPr activation by both established and novel KOPr agonists modulates $5 \mathrm{HT}$ systems. 


\subsubsection{Live cell ASP ${ }^{+}$uptake accumulation model}

4-(4-(dimethylamino)-styryl)-N-methylpyridinium $\left(\mathrm{ASP}^{+}\right)$, an analogue of the neurotoxin 1-methyl-4-phenylpyridinium (Javitch et al., 1985) is a monoamine transporter substrate which fluoresces in a lipid environment (Schwartz et al., 2003; 2005; Blakely and DeFelice, 2007). $\mathrm{ASP}^{+}$has been used to measure real-time function of DAT (Bolan et al., 2007; Zapata et al., 2007), NET (Schwartz et al., 2003) and SERT (Oz et al., 2010) in single cells. The transport of $\mathrm{ASP}^{+}$can be classified into two stages. Initially, $\mathrm{ASP}^{+}$binds rapidly to the monoamine transporter which is followed by its linear uptake into the cell. This can be visualized as accumulation of $\mathrm{ASP}^{+}$fluorescence within the cell (Schwartz et al., 2005; Fig. 3.1). Recent reports have shown that $\mathrm{ASP}^{+}$ binds to SERT at micromolar concentrations and accumulates in cells expressing SERT (Schwartz et al., 2003; Oz et al., 2010). Additionally, the uptake of $\mathrm{ASP}^{+}$is saturable, temperature dependent, and is dependent on sodium and chloride ions (Schwartz et al., 2003; Oz et al., 2010). Radio-ligand uptake assays are usually employed to measure SERT function (Owens et al., 2001). The advantage of using the $\mathrm{ASP}^{+}$uptake technique is that modulation of SERT function can be observed in real-time, whereas this cannot be done by using the radio-ligand uptake assays (Oz et al., 2010). This technique can also determine alterations in transporter functional kinetics using single cells over a period of time, whereas radio-ligand assays require addition of ligands followed by washout periods (Bylund and Toews, 1993) which limits the collection of rapid kinetic data. Within cell- and between cell- $\mathrm{ASP}^{+}$uptake protocols can be used to determine monoamine transporter function immediately or after incubating cells with drug treatment (Oz et al., 2010; Bolan et al., 2007; Zapata et al., 2007; Schwartz et al., 2003). The rationale behind this study was to determine if U50488H, Sal A and DS1 had the ability to directly modulate SERT function in vitro in cells transiently expressing both SERT and KOPr. Development of an in vitro cell model will help to determine the 
mechanism by which KOPr agonists modulate addictive behaviours. Overall, this may aid in the development of more effective and better tolerated anti-addiction pharmacotherapies.

Results from the previous chapter (Chapter 2) indicate that Sal A, DS1, MOM Sal B and nalmefene attenuate cocaine prime induced drug-seeking, an effect similar to what has been reported using the traditional KOPr agonists. Therefore, this chapter deals with studying the adverse behavioural effect profile of these compounds with respect to motor function, CTA and FST in rats. The 5HT system is implicated in the aetiology and treatment of depression (Pare, 1971; Pare et al., 1974). It is therefore possible that KOPr agonists induced depressive like symptoms might improve via modulating the serotonin systems. In order to test this, the effect of antagonising SERT function prior to novel KOPr agonist exposure on depressive like behaviours were measured using FST in rats. Additionally, we tested the effect of pre-treatment with established as well as novel KOPr agonists on modulating SERT function using live cell $\mathrm{ASP}^{+}$accumulation assay in vitro.

\subsubsection{Aims}

KOPr compounds (Sal A, DS1, MOM Sal B, nalmefene) with demonstrated efficacy for attenuating cocaine seeking behaviours as identified in Chapter 2, were tested for undesirable behavioural side-effects. The aims of the experiments described in this chapter were:-

1. To determine if selected KOPr compounds (Sal A, DS1, MOM Sal B, nalmefene) alter cocaine induced hyperactivity in cocaine self-administering rats (motor suppression during reinstatement tests).

2. To determine if single exposure to KOPr agonists (Sal A, DS1, MOM Sal B and nalmefene) alters the rats natural reward-seeking behaviour. This will be done by 
measuring weather $\mathrm{KOPr}$ agonists disrupt operant responding to obtain $10 \%$ sucrose solution.

3. To determine if acute exposure to Sal A, DS1, MOM Sal B and nalmefene causes motor suppression (sedation) during recording of spontaneous open field activity.

4. To determine if acute exposure to Sal A, DS1 and nalmefene produces aversion using CTA in rats.

5. To determine if acute exposure to Sal A, DS1 and nalmefene produces depressive symptoms using the FST in rats.

6. To identify if SERT blockade improves KOPr agonist induced depressive like behaviour. In order to determine this, the effect of sub-chronic treatment with FLX on Sal A and DS1 induced depressive like behaviours will be determined by using the FST paradigm in rats.

7. To study the effect of classical KOPr (U50488H) and novel neoclerodane diterpenes (Sal A, DS1) KOPr agonists on modulating SERT function using live cell $\mathrm{ASP}^{+}$uptake assay in vitro.

\section{$\underline{3.2 \text { Methods }}$}

\subsubsection{Subjects}

Animals previously self-administering cocaine and used in cocaine reinstatement tests were also used to measure cocaine induced hyperactivity. These were male Sprague Dawley rats, weighing 350-400 g. Drug naive male Sprague Dawley rats weighing 200-300 g were used for the spontaneous open field activity, CTA, FST and sucrose reinforcement tests. In addition rats (Male Sprague Dawley; 350-400 g) which were self-administering and tested for reinstatement but no longer had patent iv 
cannulae were used for preliminary experiments with the KOPr compounds for the FST (Tab. 3.1).

Animals were housed individually (cocaine induced hyperactivity in selfadministering rats, preliminary experiments with FST, sucrose reinforcements, CTA) or two per cage (spontaneous locomotion, FST in drug naive rats) in hanging polycarbonate cages. All rats were housed at least 5 days prior to the test in the animal facility in a temperature $\left(19-21^{\circ} \mathrm{C}\right)$ and humidity controlled $(55 \%$ relative humidity) room. Animals were handled for at least 5 days before the experiments to avoid handling stress. Lights in the room were maintained at 12:12 h, with lights on from 0700 h. All experimental procedures were approved by the Animal Ethics Committee of Victoria University of Wellington.

For locomotion tests, rats were placed in the activity chambers and were acclimatized for $30 \mathrm{~min}$ before they received drug treatment. For sucrose reinforcement tests, animals were maintained at approximately $85 \%$ of their initial feeding weights with free access to water throughout the experiment. For conditioned taste aversion, rats had free access to food but were water deprived for $23 \mathrm{hr}$ (water habituation session) or $23 \mathrm{hr} 20 \mathrm{~min}$ (saccharin sessions) per day for the entire duration of study. During habituation sessions, rats received water, whereas, on the pairing and test days, animals received a $0.1 \%$ novel tasting saccharin solution. Doses used to test the undesirable effects for each KOPr agonist (Sal A, DS1, MOM Sal B, nalmefene) were the minimal effective dose which attenuated cocaine reinstatement $(0.3 \mathrm{mg} / \mathrm{kg}$; Chapter 2$)$. 
Tab 3.1. Amount of cocaine consumed by rats used for preliminary FST experiments.

\begin{tabular}{|c|c|c|c|}
\hline $\begin{array}{c}\text { Sr. } \\
\text { No. }\end{array}$ & $\begin{array}{c}\text { Days exposed to } \\
\text { cocaine (days) }\end{array}$ & $\begin{array}{c}\text { Days abstinent from } \\
\text { cocaine before FST } \\
\text { (days) }\end{array}$ & $\begin{array}{c}\text { Amount of cocaine } \\
\text { consumed (mg/kg) }\end{array}$ \\
\hline 1 & 82 & 26 & 564 \\
\hline 2 & 76 & 22 & 392 \\
\hline 3 & 81 & 18 & 393 \\
\hline 4 & 77 & 26 & 380 \\
\hline 5 & 77 & 30 & 510 \\
\hline 6 & 76 & 32 & 547 \\
\hline 7 & 74 & 27 & 601 \\
\hline 8 & 78 & 32 & 487 \\
\hline 9 & 78 & 34 & 568 \\
\hline 10 & 61 & 33 & 649 \\
\hline 11 & 59 & 33 & 359 \\
\hline Avg & $\mathbf{7 5}$ & $\mathbf{2 9}$ & $\mathbf{4 9 6}$ \\
\hline
\end{tabular}




\subsubsection{Cell culture maintenance for live cell $A S P^{+}$uptake experiments.}

HEK-293 (American type culture collection, USA) cells were maintained in culture in Dulbecco's Modified Eagle Medium (Gibco, Invitrogen) containing 10\% foetal bovine serum (Gibco, Invitrogen) and $1 \%$ penstrap (5,000 units of penicillin $G$ sodium salt and 5,000 $\mu \mathrm{g}$ streptomycin sulphate $/ \mathrm{ml}$ in $0.85 \%$ saline; Invitrogen). The cells were grown in a T75 tissue culture flask (BD Bioscience) and were maintained in a humidified atmosphere in an incubator at $37^{\circ} \mathrm{C}$ with $5 \% \mathrm{CO}_{2}$.

\subsubsection{Drugs}

Sal A, DS1 and MOM Sal B (from Dr. Thomas E. Prisinzano, University of Kansas) were suspended in 75\% DMSO. Cocaine hydrochloride (Merck, Palmerston North, New Zealand), nalmefene (Sigma Aldrich, MO) and fluoxetine (Tocris, UK) were dissolved in $0.9 \%$ saline and ip and sc injections were administered in the volume of $1 \mathrm{~mL} / \mathrm{kg}$. For live cell confocal microscopy experiments, 4-(4-(dimethylamino)styryl)-N-methylpyridinium ( $\mathrm{ASP}^{+}$; Sigma Aldrich, St Louis MO) was dissolved in Krebs Ringer Herpes buffer (KRH; pH 7.4). U50488H (Sigma Aldrich, St. Louis, MO), Sal A and DS1 were initially suspended in 100\% DMSO followed by further dilution into $\mathrm{KRH}(\mathrm{pH} 7.4)$.

\subsubsection{Apparatus}

\subsubsection{Locomotion tests}

As described in Section 2.2.4.1, Chapter 2.

\subsubsection{Sucrose reinforcement}

Training and testing procedures were conducted in eight modular test chambers (Med Associates, ENV-008) equipped with two retractable levers (Med Associates ENV-112CM) situated at the front of the chamber to either side. $10 \%$ sucrose solution was delivered by a liquid dipper (Med-Associates ENV-202M). The dipper was situated 
in the centre, at the front of the chamber $2 \mathrm{~cm}$ from the chamber floor. The two retractable levers were positioned to the left and right $8 \mathrm{~cm}$ from the liquid dipper and 3 $\mathrm{cm}$ from the sides of the chamber. These chambers were located in an unlit, sound attenuated room. Sucrose delivery and data acquisition were controlled by Med Associates software (St Albans, VA, USA). Experiments were conducted between 0900 and $1600 \mathrm{hr}$.

\subsubsection{Forced swim test}

The FST apparatus consisted of a cylinder which was $44 \mathrm{~cm}$ high and $20 \mathrm{~cm}$ in diameter. The cylinder was filled with water maintained at $25 \pm 1{ }^{\circ} \mathrm{C}$. The water was filled up to $35 \mathrm{~cm}$ and the animal was placed in the water and behaviour was recorded by a camera connected to a nearby computer. The forced swimming behaviours (climbing, swimming or immobile) were recorded for a period of $5 \mathrm{~min}$ and later analysed by a blinded to treatment observer.

\subsubsection{Procedure}

\subsubsection{Cocaine induced hyperactivity (in self-administering rats)}

This test was conducted to measure weather KOPr agonists caused any disruption in motor function during the reinstatement test. Methods described by Morani et al., (2009) were followed. The conditions for this test were maintained similar to the reinstatement test. On the test day, rats previously self-administering cocaine received an injection of either Sal A (0.3 mg/kg, ip) $5 \mathrm{~min}, \mathrm{MOM} \mathrm{Sal} \mathrm{B}(0.3 \mathrm{mg} / \mathrm{kg}$, ip) $5 \mathrm{~min}$, nalmefene $(0.3$ $\mathrm{mg} / \mathrm{kg}, \mathrm{sc}) 15 \mathrm{~min}$ and DS1 (0.3 mg/kg, ip) $45 \mathrm{~min}$ prior to an injection of cocaine (20 $\mathrm{mg} / \mathrm{kg}$, ip). Immediately following the cocaine injection, the rats were placed in the activity chambers and total activity, a compilation of horizontal and vertical activity, and ambulation at 5 min intervals was measured for a period of $1 \mathrm{hr}$. All injections were administered in the open field chamber. The experimenter remained outside the test room while the activity test was in progress. 


\subsubsection{Spontaneous open field activity (drug naive rats)}

This test was performed to detect any sedative or motor suppression effects produced by an acute exposure to the KOPr agonists. For this study, we modified the methods of Frankowska et al, (2009) and Xu et al, (2010). On the test day, drug naive rats were injected with either vehicle (75\% DMSO or $0.9 \%$ saline) or KOPr compounds $5 \mathrm{~min}$ (Sal A, $0.3 \mathrm{mg} / \mathrm{kg}$, ip; MOM Sal B, $0.3 \mathrm{mg} / \mathrm{kg}$, ip), $15 \mathrm{~min}$ (nalmefene, $0.3 \mathrm{mg} / \mathrm{kg}$, sc) or $45 \mathrm{~min}$ (DS1, $0.3 \mathrm{mg} / \mathrm{kg}$, ip) prior to measuring their spontaneous open field activity. Total activity, a compilation of horizontal and vertical activity, and ambulation at 5 min intervals was measured for a period of $1 \mathrm{hr}$. All injections were administered in the activity chamber with the experimenter remaining outside the test room while the activity test was in progress.

\subsubsection{Sucrose reinforcement training and test}

This test was performed to determine the effect of acute exposure to KOPr agonists on operant lever press responding to a palatable reward (10\% sucrose solution). Methods described by Morani et al., (2009) were followed. Animals were maintained at approximately $85 \%$ of their initial feeding weights throughout the experiment. Initially rats were trained to obtain sucrose reinforcements using an auto-shaping procedure for 45 min daily for ten consecutive days. Once they were stably responding, the animals were trained to self-administer sucrose orally on a FR1 schedule of reinforcement. Following acquisition, the response requirement was increased to a FR-5 schedule. Daily $1 \mathrm{hr}$ sessions were conducted until there was less than $20 \%$ variation in responding for three consecutive days. Once responding on the FR-5 schedule was stable, the effects of prior administration of Sal A $(0.3,1.0 \mathrm{mg} / \mathrm{kg}$, ip), DS1 $(0.3,1.0$ $\mathrm{mg} / \mathrm{kg}$, ip), MOM Sal B (0.3 mg/kg, ip), nalmefene $(0.3 \mathrm{mg} / \mathrm{kg}, \mathrm{sc})$ and vehicle $(75 \%$ DMSO; $0.9 \%$ saline) on sucrose-reinforced responding were noted. The injections were administered $5 \mathrm{~min}$ (Sal A, MOM Sal B), 15 min (nalmefene) and $45 \mathrm{~min}$ (DS1) prior to 
the experiment and responding was measured for $60 \mathrm{~min}$ following drug/vehicle treatment.

\subsubsection{Conditioned taste aversion}

This test was performed to determine the aversive effects produced by a single injection of each of the KOPr agonists. To do this we measured the amount of novel tasting saccharin solution (which had been paired with KOPr agonist) consumed on test day. For this experiment, we modified methods described by Fenu et al., (2005) and Schenk et al., (1987).

Habituation: On day 1 of the experiment the rats were placed on a water deprivation schedule for $23 \mathrm{~h}$. The following day, animals were given access to water for $1 \mathrm{~h}$. The amount of water consumed was measured on a daily basis, to the nearest $\mathrm{mL}$. This process was repeated untill the variation in water consumption was $\leq 2 \mathrm{~mL}$ for three consecutive days.

Pairing: The day after rats attained stable water consumption, they were subjected to pairing session. During these sessions, rats were presented with a novel $0.1 \%$ saccharin solution instead of water, and the amount of saccharin consumed $(\mathrm{mL})$ was noted for 40 min. The animals were divided evenly into two groups (Vehicle or KOPr agonist treated), matched on saccharin intake and were immediately injected with either vehicle (75\% DMSO or $0.9 \%$ saline) or KOPr compound (Sal A, DS1 or nalmefene) and were returned to their home cage. The following day ( $24 \mathrm{~h}$ post pairing sessions), rats were provided with drinking water for $60 \mathrm{~min}$ and were tested for the saccharin aversion the next day (24 h later).

Test day: $48 \mathrm{hr}$ after the pairing session, rats were again presented with the saccharin solution and the amount of saccharin consumed was measured for $40 \mathrm{~min}$ (to the nearest $\mathrm{mL}$ ). In order to determine any taste aversion produced by the novel KOPr compound, the amount of saccharin consumed on the pairing day and test day by the KOPr agonist 
treated groups was compared to the corresponding vehicle treated group. All experiments were performed between $1100-1300 \mathrm{hr}$.

\subsubsection{FST in drug naïve rats and preliminary experiments.}

To initially screen the depressive effect of novel KOPr agonists, FST was carried out using animals which were previously subjected to self-administration training and reinstatement tests (Tab 3.1). These rats were withdrawn from the selfadministration experiments due to the failure of their jugular cannulae. The average total amount of cocaine consumed by these rats was $496 \mathrm{mg} / \mathrm{kg}$ (range: $359-649 \mathrm{mg} / \mathrm{kg}$ ). The average number of days these rats were exposed to cocaine was 75 days (range: 5982 days). The average number of days they were abstained from cocaine was 29 (range: 18-34 days). Depressive effects produced by KOPr agonists in preliminary tests were later determined in drug naive animals. For this experiment, we modified methods of Carlezon et al., (2006).

Habituation day: Animals were habituated in the test room for $60 \mathrm{~min}$ prior to the commencement of experiments. Rats were singly placed in the FST chamber and habituated to forced swimming for $15 \mathrm{~min}$. Rats initially tried to escape from the FST chamber but later they habituated to the swimming conditions which was observed as maintaining immobility. After the 15 min session, animals were removed from the chamber, dried using cloth towels and were placed under a heating lamp for at least 30 min. Water in the cylinder was changed every 1-2 rats (to remove animal litter). After the habituation sessions were completed, the rats were returned to their home cages.

Test day: Rats were injected with KOPr compounds 5 min (Sal A), 15 min (nalmefene) and 45 min (DS1) prior to the experiments. This second FST was carried out for a period of $5 \mathrm{~min}$. The climbing, swimming and immobility behaviours were recorded using a camera connected to a nearby computer via Ethernet. To avoid any bias in 
analysis, treatments were masked from the experimenter during subsequent video analysis. The incidence of each FST behaviour was analysed in bins of $5 \mathrm{sec}$ interval.

\subsubsection{Effect of serotonin transporter blockade by FLX on KOPr agonist induced depression in FST:}

The habituation and test sessions were carried out as previously described (section 3.2.4.5). In between the habituation and test sessions, rats were treated with saline (1 mL/kg, sc) or FLX (5 mg/kg, sc; Detke et al., 1995; Estrada-Camarena et al., 2003), $30 \mathrm{~min}, 19 \mathrm{hr}$ and $23 \mathrm{hr}$ post habituation. The next day (24 hr after habituation), animals received the KOPr agonists (Sal A, DS1) and were subjected to FST for 5 min. The data obtained from these experiments were analysed as described in section 3.2.5.5.

\subsubsection{Transfections and live-cell imaging to determine SERT function modulation} by KOPr agonists (between cell designs).

Transfections:- For $\mathrm{ASP}^{+}$uptake experiments, HEK-293 cells were trypinised (TrypLETM express stable Trypsin-like enzyme with Phenol red, Invitrogen, New Zealand), washed and cultured in fresh antibiotic-free media (Dulbecco's modified Eagle medium (Gibco, Invitrogen) containing 10\% fetal bovine serum) and were seeded in $35 \mathrm{~mm}$ sterile glass bottomed Delta T culture dishes (MatTek MA, USA) at 1.25 X $10^{5}$ cells $/ \mathrm{mL}$, using $1 \mathrm{~mL} /$ dish. Transient transfections were performed with plasmids containing green fluorescence protein tagged human SERT (GFP-hSERT; a gift from Dr. Samanda Ramamoorthy, Medical University of South Carolina, USA; Ramamoorthy et al., 1993) and myc-tagged rat KOPr (myc-rKOPr; a gift from Dr. Lakshmi Devi, Mount Sinai School of Medicine, NY, USA; Li et al., 1993; Meng et al., 1993) using Lipofectamine 2000 (Invitrogen) and OptiMEM media (Invitrogen). The transfections were carried out according to the manufacturer's instructions (Appendix 3). The transfection solution contained $3 \mu \mathrm{L}$ Lipofectamine and $1 \mu \mathrm{g}$ plasmid DNA (GFP- 
hSERT: $0.4 \mu \mathrm{g}$; myc-rKOPr: $0.6 \mu \mathrm{g}$ ) and the volume made up to $100 \mu \mathrm{l}$ using OptiMEM. Each $35 \mathrm{~mm}$ culture dish was treated with $100 \mu \mathrm{L}$ of the transfection solution. The cells were transfected $24 \mathrm{hr}$ post-seeding. Drug treatment and imaging experiments were performed 36-48 hr post transfection when cells were found confluent between $70-90 \%$.

Live-cell ASP${ }^{+}$uptake experiments:- On the day of the experiment, cells were treated with either drug $(10 \mu \mathrm{M}$ U50488H, $10 \mu \mathrm{M}$ Sal A, $10 \mu \mathrm{M}$ DS1) or vehicle (0.1\% DMSO diluted in Krebs Ringer Herpes buffer; KRH, pH: 7.4, Appendix 4) and placed into the incubator for $30 \mathrm{~min}$. Just before image capture, the media from the dish was replaced with $\mathrm{KRH}$ and the dish was mounted on a temperature $\left(37^{\circ} \mathrm{C}\right)$ and humidity controlled stage (Tokai Hit; INU-ZILCS-Fl; Shizuoka-ken; Japan) and attached to an Olympus FV1000 laser scanning confocal microscope (Olympus, Wellington, New Zealand). Initially cells were visualized using a 40X water immersion objective (Olympus) and a monolayer of cells selected. The KRH was then removed without disturbing the dish and an initial image showing GFP-hSERT fluorescence was captured (Fig. 3.1A). Immediately after this, $1 \mathrm{ml}$ of $10 \mu \mathrm{M} \mathrm{ASP}^{+}$solution in $\mathrm{KRH}$ (Sigma, $\mathrm{MO} ; 1 \mathrm{~mL} / \mathrm{dish}$ ) was added, and live-cell $\mathrm{ASP}^{+}$uptake recorded for 5 min with images taken every $5 \mathrm{sec}$ (Fig. 3.1B). The GFP-hSERT and $\mathrm{ASP}^{+}$were excited to fluoresce by using the solid state lasers (Olympus, Wellington, New Zealand). GFP-hSERT was excited at $473 \mathrm{~nm}$, (485-545 filter) and $\mathrm{ASP}^{+}$was excited at $559 \mathrm{~nm}$ (570-670 filter).

Data analysis for live-cell ASP ${ }^{+}$uptake experiments:- Individual cells were selected in a region of interest using both differential interference contrast and GFP-hSERT images to localise the cell boundaries (Fig. 3.1C). The GFP-hSERT and $\mathrm{ASP}^{+}$fluorescence were quantified for each cell using Fluoview FV1000 software (Olympus; Fig. 3.1D). 
Non-transfected cells had shown some non-specific $\mathrm{ASP}^{+}$accumulation. This nonspecific accumulation was subtracted from the accumulation measured in the transfected cells. In order for $\mathrm{ASP}^{+}$accumulation to be directly correlated to the amount of GFPhSERT expressed on the cell surface, we expressed $\mathrm{ASP}^{+}$uptake as a percentage of SERT expression using the following formula:-

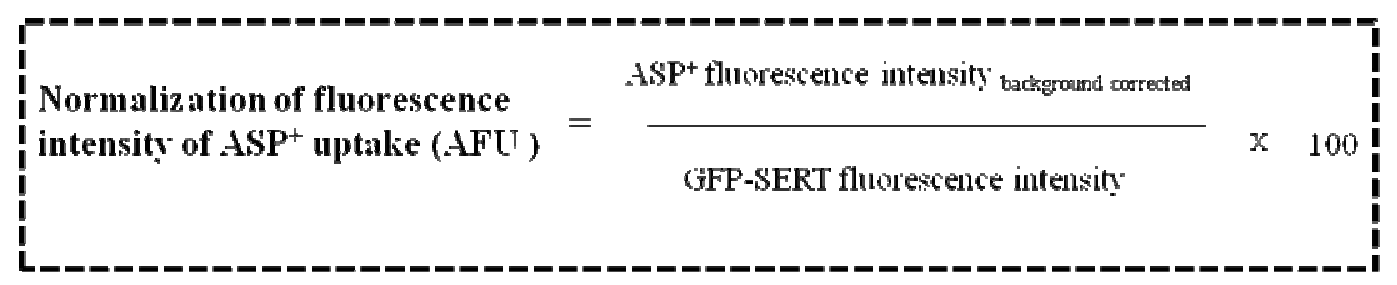

All experiments were performed in duplicate from at least three separate transfections. 

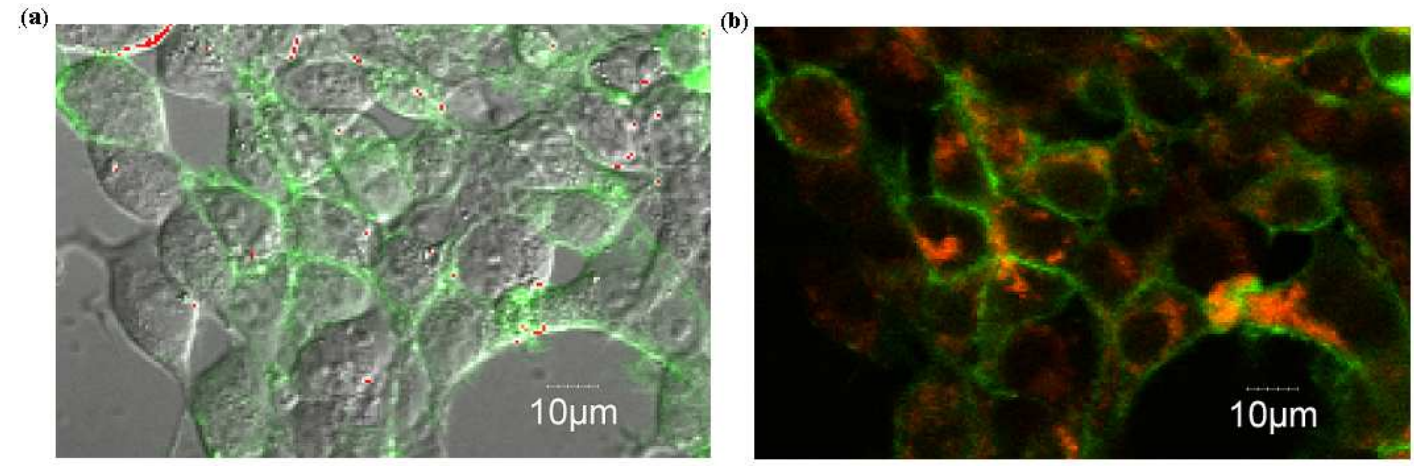

(c)

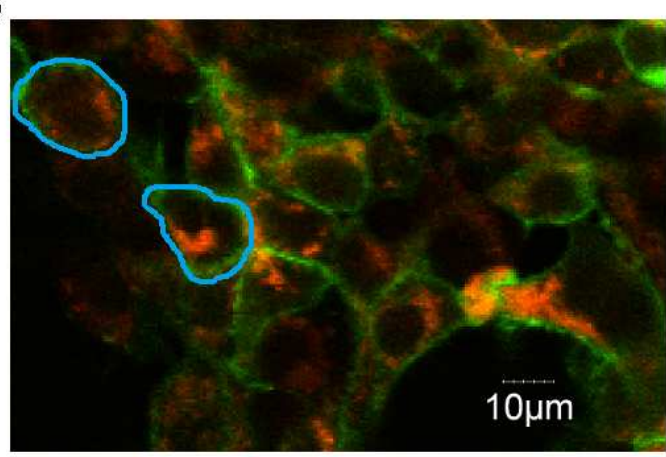

(d)

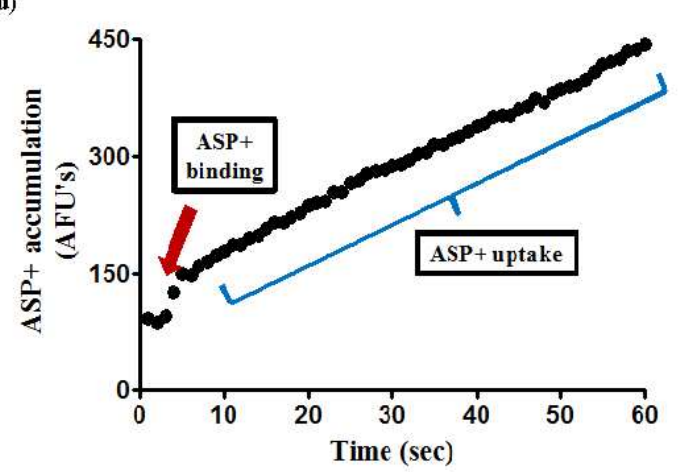

\section{Fig. 3.1 ASP+ accumulation and fluorescence analysis.}

A. After incubating the cells with KOPr agonists (10 $\mu M$ U50488H, Sal A, DS1) or vehicle (DMSO), each culture dish was placed on the inverted confocal microscope stage which focussed on the monolayer of cells visualised using differential interference contrast and GFPhSERT fluorescence (green colour) to identify and locate cells. The GFP-hSERT fluorescence was captured just before adding $A S P^{+}$. B. ASP ${ }^{+}$was then added to the dish and uptake was measured every $5 \mathrm{sec}$ for $5 \mathrm{~min} . \boldsymbol{C}$. After 5 min uptake, cells were circled and the amount of $A S P^{+}$uptake accumulated (red colour) over 5 min was determined using Fluoview software (Version 2.0c). D. The binding to and uptake pattern of $A S P^{+}$in a representative GFP-hSERT and myc-rKOPr transfected cell. Scale bar represents $10 \mu \mathrm{m}$. 


\subsubsection{Statistical analysis}

Data analysis was performed using Prism Graphpad software (Version 5.0; San Diego, CA). Statistical analysis for cocaine induced hyperactivity; spontaneous open field activity; sucrose reinforcements (for MOM Sal B, nalmefene) were performed using separate Student t-tests. The sucrose reinforcement tests for Sal A, DS1 and CTA (for Sal A, DS1 and nalmefene) were analysed using one way ANOVA followed by Tukey post-hoc tests. The effect of 5HT system modulation via SERT blockade using FLX on Sal A and DS1 induced immobility were analysed using a separate two-way ANOVA for each FST behaviour'. For comparing FST results in cocaine exposed and drug naïve rats, each forced swimming behaviour' was analysed using the MannWhitney test. Data for correlation experiments measuring $\mathrm{ASP}^{+}$uptake and GFPhSERT expression levels were analysed using linear regression. $\mathrm{ASP}^{+}$accumulation experiments in non-transfected vs. transfected cells were analysed from the $5 \mathrm{~min}$ uptake values using student t-test. Changes in $\mathrm{ASP}^{+}$uptake in KOPr treated cells were analysed using one-way ANOVA followed by Dunnets multiple comparison tests (Oz et al., 2010). For analysing GFP-hSERT expression levels in cells selected for $\mathrm{ASP}^{+}$ accumulation experiments, one-way ANOVA followed by Dunnets multiple comparison tests were performed. 


\subsection{Results}

\subsubsection{Effect of KOPr compounds on cocaine induced hyperactivity (cocaine self- administering rats).}

The effect of Sal A (0, $0.3 \mathrm{mg} / \mathrm{kg}$, ip), DS1 $(0,0.3 \mathrm{mg} / \mathrm{kg}$, ip), MOM Sal B(0, $0.3 \mathrm{mg} / \mathrm{kg}$, ip) and nalmefene $(0,0.3 \mathrm{mg} / \mathrm{kg}, \mathrm{sc})$ pre-treatment on cocaine induced hyperactivity in rats previously self-administering cocaine and subjected to reinstatement tests is shown in Fig 3.2. No significant reduction in cocaine produced hyperactivity was observed in either Sal A ( $p=0.46$; Fig 3.2a) and MOM Sal B pretreated animals $(\mathrm{p}=0.43$; Fig 3.2c). A non-significant trend to increase total activity was noted in animals treated with nalmefene $(p=0.216$; Fig $3.2 \mathrm{~d})$. However, rats that were pre-treated with DS1 showed a non-significant trend towards a decrease in cocaine produced hyperactivity $(\mathrm{p}=0.09 ;$ Fig $3.2 \mathrm{~b})$. 
(a)

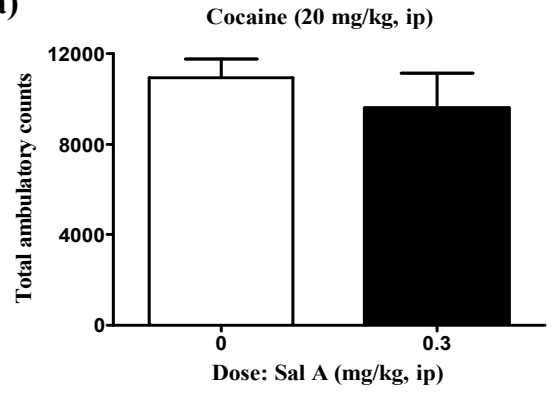

(b)

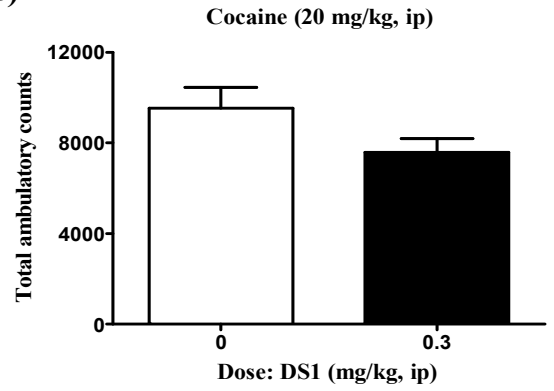

(c)

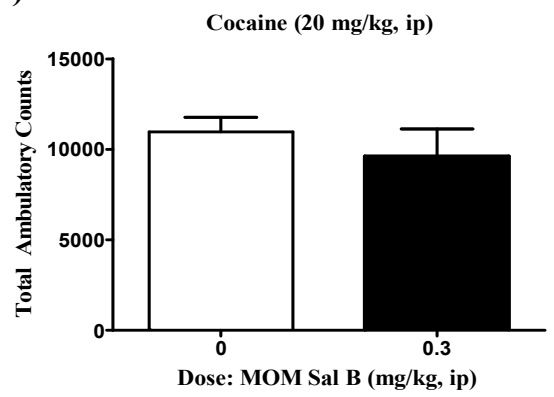

(d)

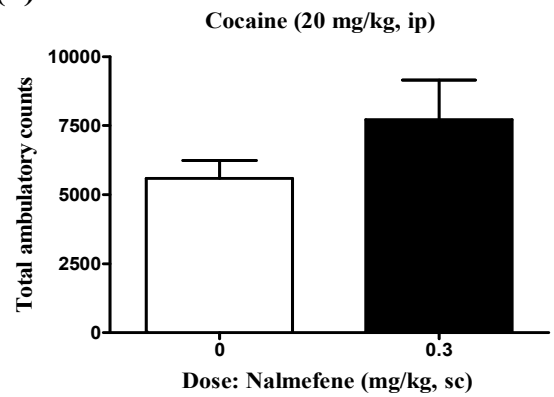

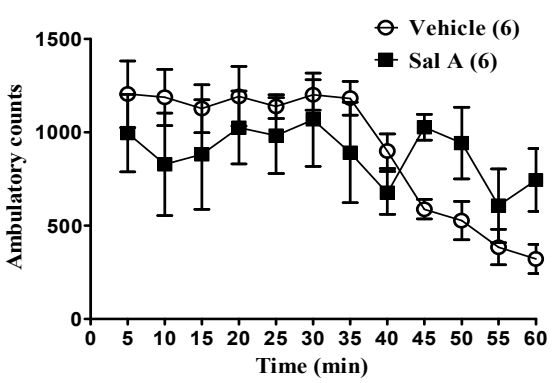
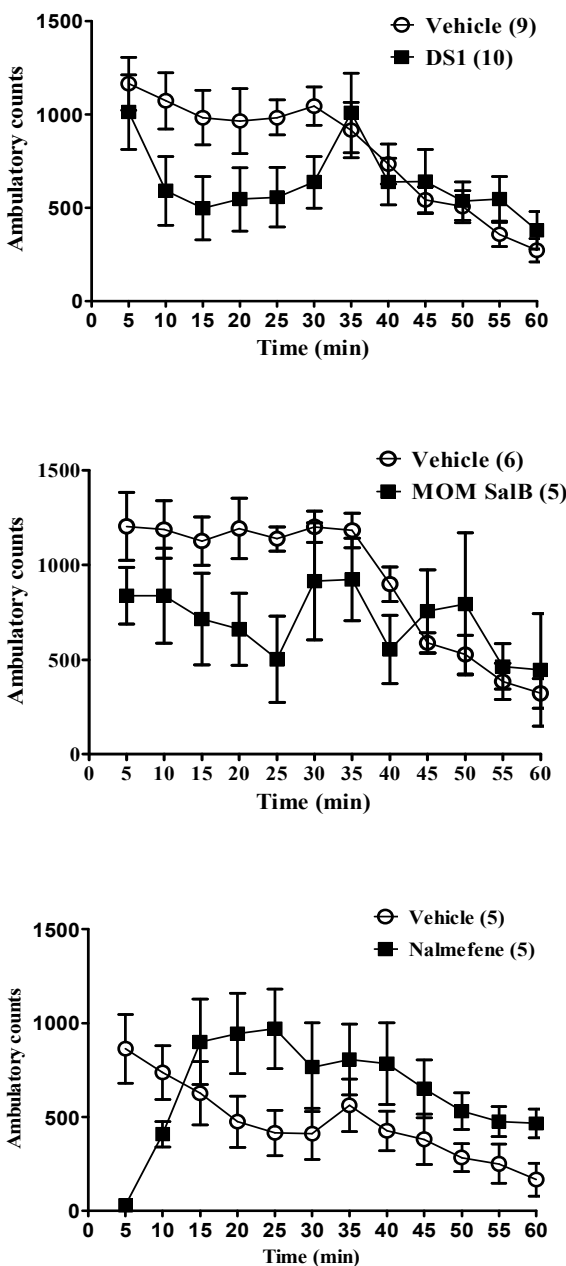

Fig 3.2. Effect of KOPr compounds on cocaine induced hyperactivity (cocaine self-administering rats).

Rats previously self-administering cocaine were initially treated with either (a) Sal A (0, 0.3 $\mathrm{mg} / \mathrm{kg} ; n=6)$, (b) DS1 (0, $0.3 \mathrm{mg} / \mathrm{kg} ; n=9,10)$, (c) MOM Sal B (0, $0.3 \mathrm{mg} / \mathrm{kg} ; n=5,6),(d)$ nalmefene ( $0,0.3 \mathrm{mg} / \mathrm{kg} ; \mathrm{n}=5)$. This was followed by a priming injection of cocaine (20 $\mathrm{mg} / \mathrm{kg})$ and total locomotion was measured for $60 \mathrm{~min}$. Symbols in the left panel represent total ambulatory counts (+SEM) and in right panel indicates mean ( \pm SEM) of ambulation measured every 5 min interval. Student $t$-test. 


\subsubsection{Effect of KOPr compounds on natural reward reinforcements}

The effect of Sal A $(0,0.3,1.0 \mathrm{mg} / \mathrm{kg}$, ip); DS1 (0, 0.3, $1.0 \mathrm{mg} / \mathrm{kg}, \mathrm{ip}) ; \mathrm{MOM}$ Sal B $(0,0.3 \mathrm{mg} / \mathrm{kg}$, ip) and nalmefene $(0,0.3 \mathrm{mg} / \mathrm{kg}, \mathrm{sc})$ on sucrose reinforced responding is shown in Fig. 3.3. Statistical analysis indicated no significant alteration in sucrose reinforcement observed in rats which were pre-treated with either Sal A $[F(2,27)$ $=0.058, \mathrm{p}=0.944$; Fig. 3.3a $]$; DS $1[\mathrm{~F}(2,18)=1.11, \mathrm{p}=0.35$; Fig. 3.3b] and nalmefene $(\mathrm{p}>0.05$; Fig. $3.3 \mathrm{~d})$. However, a significant reduction in sucrose reinforcement was observed in rats pre-treated with MOM Sal B ( $p=0.0073$; Fig. $3.3 \mathrm{c})$. 
(a)

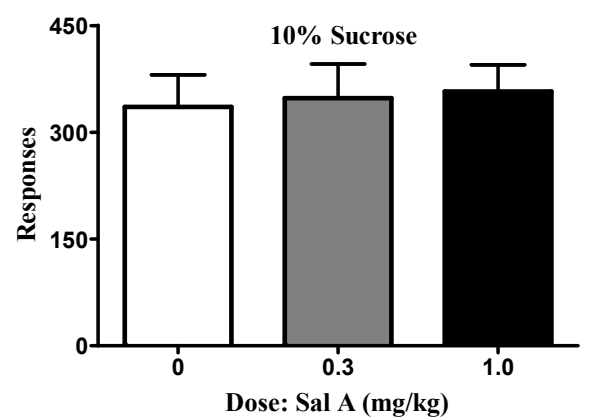

(c)

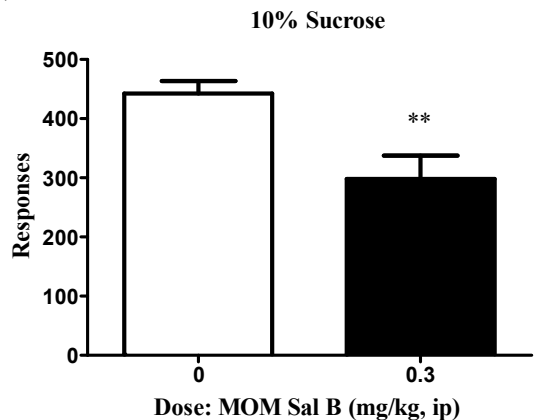

(b)

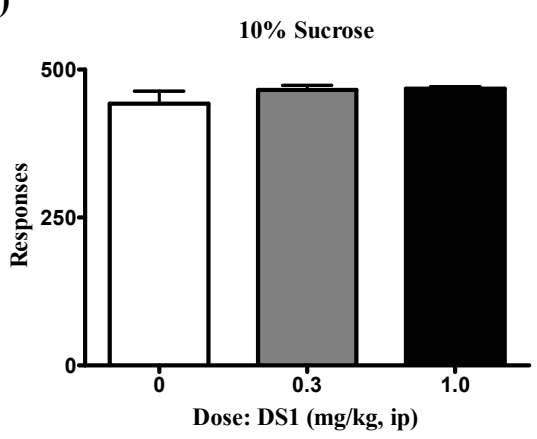

(d)

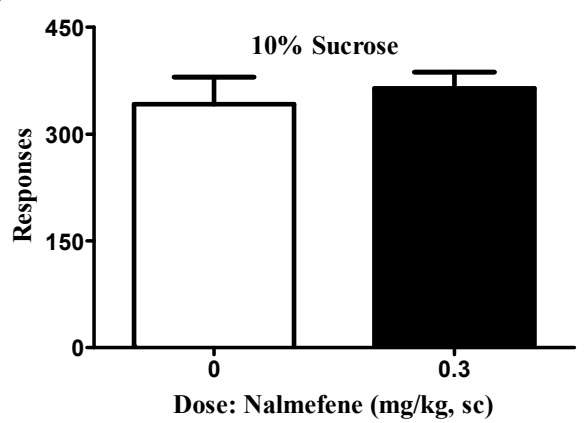

Fig. 3.3. Effect of KOPr compounds on natural reward reinforcements.

Animals which were stably responding for sucrose reinforcements at a FR-5 schedule of reinforcement were injected with KOPr compounds prior to measuring sucrose reinforced lever press responding for 60 min. Bars indicate average number of "active” lever press responses (+SEM) for 10\% sucrose solution following (a) Sal A (0, 0.3, $1.0 \mathrm{mg} / \mathrm{kg} ; \mathrm{n}=10)$, (b) DS1 (0, 0.3, $1.0 \mathrm{mg} / \mathrm{kg}, \mathrm{n=7})$, (c) MOM Sal B (0, $0.3 \mathrm{mg} / \mathrm{kg}, \mathrm{n=7})$, (d) nalmefene (0, $0.3 \mathrm{mg} / \mathrm{kg}, \mathrm{n}=6$ ) treatment. One way ANOVA followed by Tukey post-hoc tests was used for (a) and (b) and Student $t$-test for (c) and (d). ${ }^{* * p}<0.01$ compared to $0 \mathrm{mg} / \mathrm{kg}$. 


\subsubsection{Effect of KOPr compounds on motor function.}

Effect of an acute exposure to Sal A (0, $0.3 \mathrm{mg} / \mathrm{kg}$, ip); DS1 (0, $0.3 \mathrm{mg} / \mathrm{kg}$, ip); MOM Sal B $(0,0.3 \mathrm{mg} / \mathrm{kg}$, ip) and nalmefene $(0,0.3 \mathrm{mg} / \mathrm{kg}, \mathrm{sc})$ on spontaneous open field locomotion activity in rats is shown in Fig. 3.4. Statistical analysis indicated no significant change in total locomotion activity observed for Sal A ( $p=0.78$; Fig. 3.4a); DS1 ( $p=0.54$; Fig. 3.4b) and MOM Sal B ( $p=0.43$; Fig. 3.4c) pre-treated animals. However, rats pre-treated with nalmefene ( $\mathrm{p}=0.023$; Fig. $3.4 \mathrm{~d})$ significantly suppressed spontaneous open field activity. 
(a)

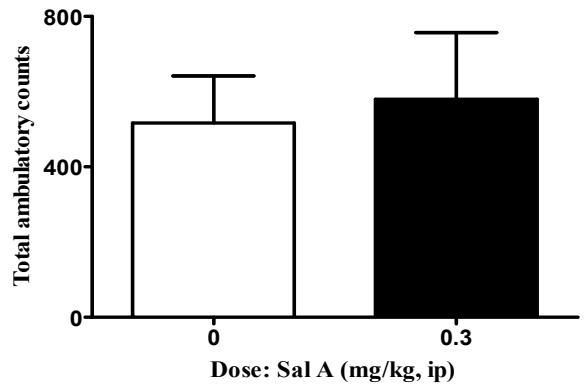

(b)

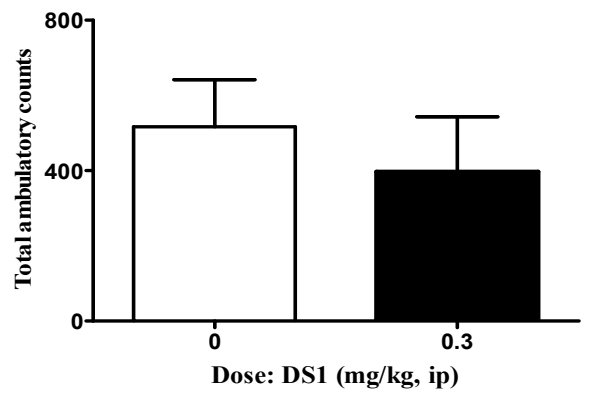

(c)

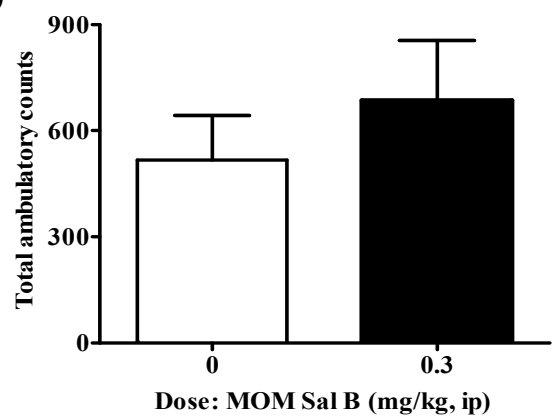

(d)

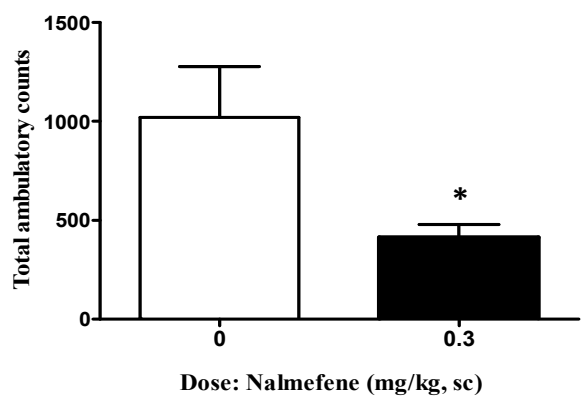

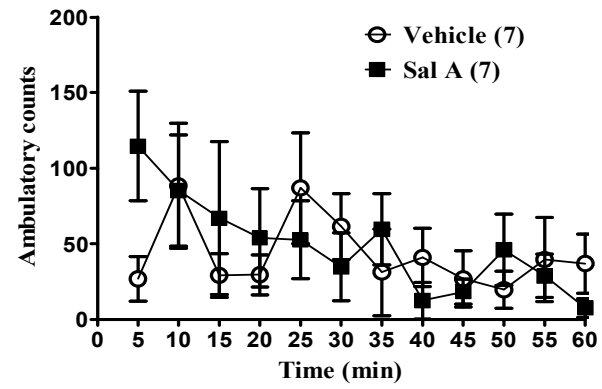
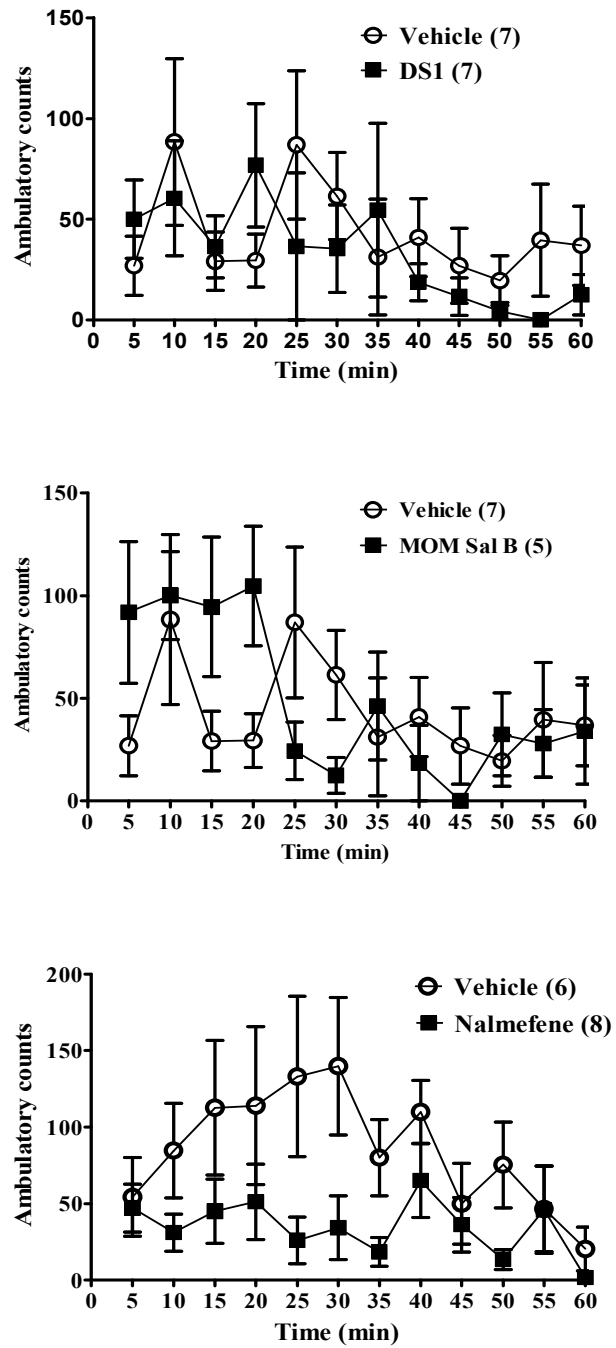

\section{Fig. 3.4. Effect of KOPr compounds on motor function.}

Drug naïve rats were treated with (a) Sal A (0, $0.3 \mathrm{mg} / \mathrm{kg} ; n=7)$, (b) DS1 (0, $0.3 \mathrm{mg} / \mathrm{kg} ; n=7)$ and (c) MOM Sal B (0, $0.3 \mathrm{mg} / \mathrm{kg} ; \mathrm{n}=5,7)$, (d) nalmefene $(0,0.3 \mathrm{mg} / \mathrm{kg}, n=5,7)$ and spontaneous open field activity was measured for $60 \mathrm{~min}$. Symbols in the left panel represent total ambulatory counts (+SEM) and in right panel indicates mean ( \pm SEM) of ambulation measured every 5 min interval. ${ }^{*} p<0.05$, data compared with $0 \mathrm{mg} / \mathrm{kg}$, Student $\mathrm{t}$-test. 


\subsubsection{Effect of KOPr compounds on conditioned taste aversion.}

The amount of saccharin consumed by rats paired with novel tasting saccharin solution and Sal A (0, $0.3 \mathrm{mg} / \mathrm{kg}, \mathrm{ip}) ; \operatorname{DS} 1(0,0.3 \mathrm{mg} / \mathrm{kg}$, ip) and nalmefene $(0,0.3$ $\mathrm{mg} / \mathrm{kg}, \mathrm{sc}$ ), on pairing day and test day is shown in Fig 3.5. Statistical analysis showed no significant difference in saccharin drinking patterns observed for Sal A $(F(3,22)=$ 2.7; $\mathrm{p}>0.05 ;$ Fig 3.5a) and nalmefene $(\mathrm{F}(3,20)=0.3 ; \mathrm{p}=0.8$; Fig 3.5c) pre-treated rats. However, DS1 pre-treated rats, showed a significant increase in saccharin drinking pattern on test day when compared with pairing day $(\mathrm{F}(3,22)=7.3 ; \mathrm{p}<0.01$; Fig $3.5 \mathrm{~b})$. 
(a)

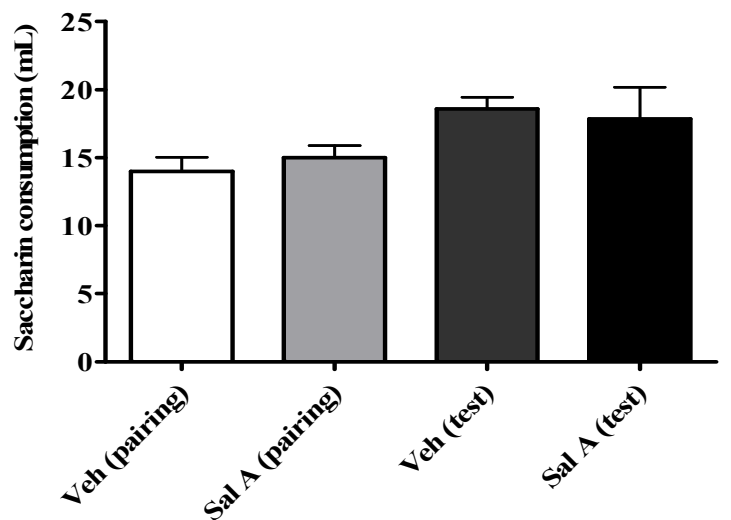

(b)

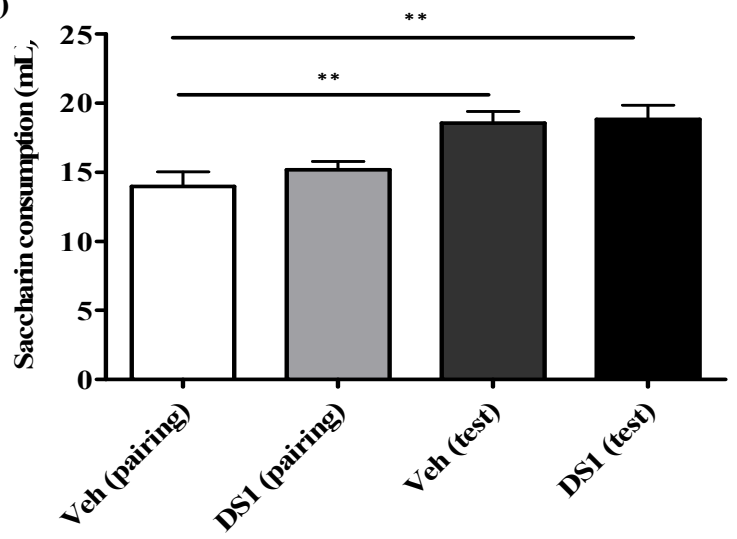

(c)

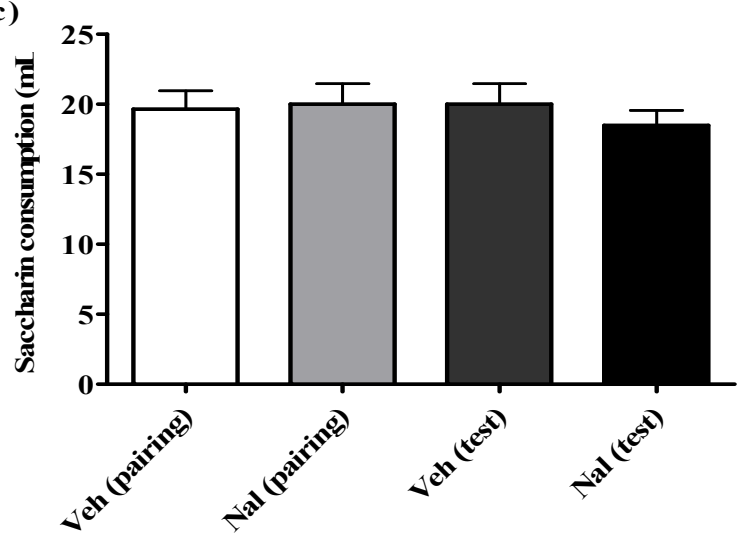

\section{Fig. 3.5. Effect of KOPr compounds on conditioned taste aversion.}

Animals experienced a pairing of a novel tasting saccharin solution and KOPr compounds [(a) Sal A (0, $0.3 \mathrm{mg} / \mathrm{kg} ; \mathrm{n}=6-7)$, (b) DS1 (0, $0.3 \mathrm{mg} / \mathrm{kg} ; \mathrm{n}=6-7)$ and (c) nalmefene (0, $0.3 \mathrm{mg} / \mathrm{kg}$; $n=6)]$. They were again presented with the saccharin solution, $48 \mathrm{hr}$ post pairing session, and the amount of saccharin consumed on pairing day and test day was measured for 40 min. Bars indicate average (+SEM) amount of $0.1 \%$ saccharin solution consumed by rats (mL) treated with vehicle or KOPr compounds on pairing and test day. **p<0.01, one way ANOVA followed by Tukey post-hoc test. 


\subsubsection{Effect of KOPr compounds on FST in rats with previous history of cocaine self-}

administration.

The effect of acute exposure to Sal A (0, $0.3 \mathrm{mg} / \mathrm{kg}$, ip); DS1 (0, $0.3 \mathrm{mg} / \mathrm{kg}, \mathrm{ip})$ or nalmefene $(0,0.3 \mathrm{mg} / \mathrm{kg}, \mathrm{sc})$ in rats with a prior history of cocaine self-administration on forced swimming behaviours are shown in Fig. 3.6.

For Sal A pre-treated animals, statistical analysis indicated no significant difference in time spent climbing $(\mathrm{p}=0.9)$ or swimming $(\mathrm{p}=0.15)$. However, a significant increase in immobility $(\mathrm{p}=0.04)$ time was observed for Sal A pre-treated rats vs. vehicle treated rats (Fig. 3.6a).

For DS1 pre-treated rats, no significant difference in time spent climbing $(p=0.83)$ was noted. However, a significant decrease in time spent swimming $(p=0.01)$ and an increase in immobility $(\mathrm{p}=0.01)$ time were noted for DS1 pre-treated rats vs. vehicle treated rats (Fig. 3.6b).

For nalmefene pre-treated rats, no significant difference in climbing time $(p=1.0)$ was noted. However, a significant reduction in swimming time $(p=0.03)$ and a significant increase in time spent immobile $(\mathrm{p}=0.02)$ were observed for nalmefene pretreated rats vs. vehicle treated rats (Fig. 3.6c). 
(a)

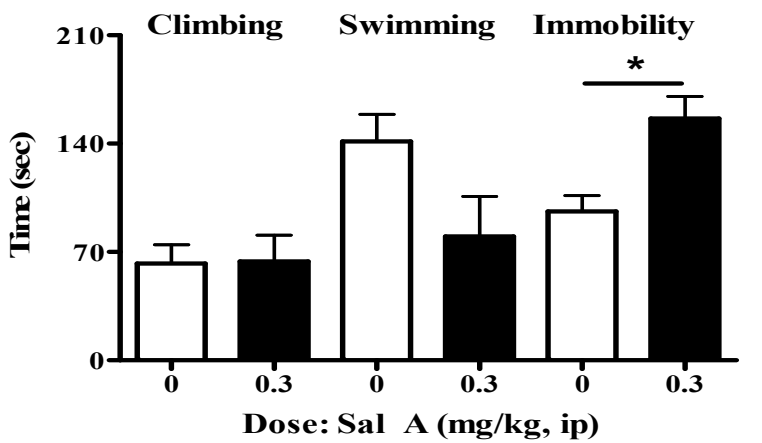

(b)

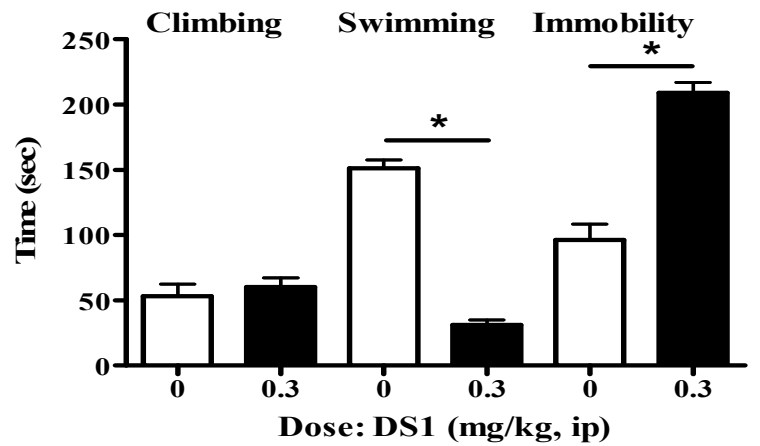

(c)

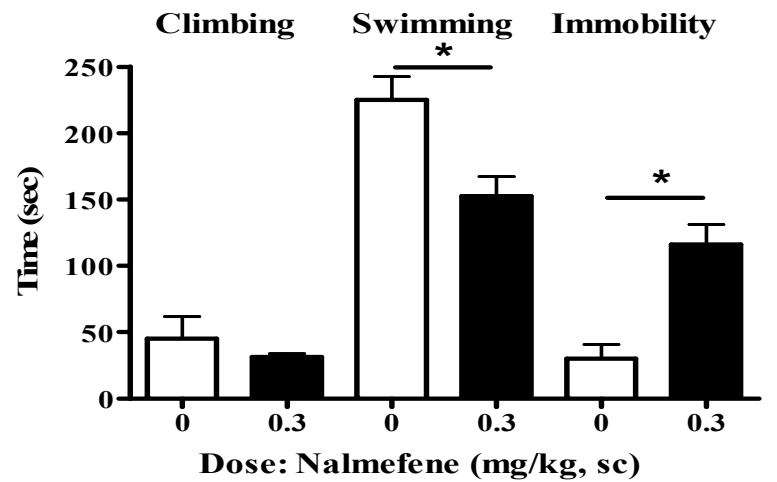

\section{Fig. 3.6. Effect of KOPr compounds on FST in rats with previous history} of cocaine self-administration.

Animals previously self-administering cocaine and subjected to reinstatement testing were habituated to the forced swimming sessions on day 1. The following day, animals received (a). Sal A (0, $0.3 \mathrm{mg} / \mathrm{kg} ; n=4),($ b). DS1 (0, $0.3 \mathrm{mg} / \mathrm{kg} ; n=5)$ or (c) nalmefene (0, $0.3 \mathrm{mg} / \mathrm{kg} ; n=4-5)$ and were subjected to FST. The forced swimming behaviours were recorded for a period of 5 min and analysed in bins of $5 \mathrm{sec}$. Data are expressed as mean time in sec (+SEM) for climbing, swimming or immobility during FST. * ${ }^{*}<0.05$. Data for $0.3 \mathrm{mg} / \mathrm{kg}$ compared with $0 \mathrm{mg} / \mathrm{kg}$ for corresponding climbing, swimming and immobile behaviours using Mann Whitney tests. 


\subsubsection{Effect of KOPr compounds on FST in drug naive rats.}

The effect of single injections of Sal A (0, $0.3 \mathrm{mg} / \mathrm{kg}$, ip); DS1 (0, $0.3 \mathrm{mg} / \mathrm{kg}$, ip) and nalmefene $(0,0.3 \mathrm{mg} / \mathrm{kg}, \mathrm{sc})$ on forced swimming behaviours in drug naive rats is shown in Fig. 3.7.

Significant reductions in climbing $(p=0.044)$ and swimming $(p=0.005)$ times were observed in rats pre-treated with Sal A. A significant increase in time spent immobile ( $\mathrm{p}=0.005)$ was also noted for Sal A vs. vehicle pre-treated animals (Fig. 3.7a).

For DS1 pre-treated animals, a non-significant trend to decrease time spent as climbing was noted $(\mathrm{p}=0.09)$. However, a significant decrease in swimming time $(p=0.006)$ and a significant increase in time spent immobile $(p=0.006)$ was observed for DS1 vs. vehicle pre-treated animals (Fig. 3.7b).

For nalmefene pre-treated rats, statistical analysis indicated no significant difference in time spent as climbing $(p=0.48)$. However, a significant decrease in swimming time $(p=0.025)$ and a significant increase in immobility time $(p=0.034)$ were observed for nalmefene vs. vehicle pre-treated animals (Fig. 3.7c). 


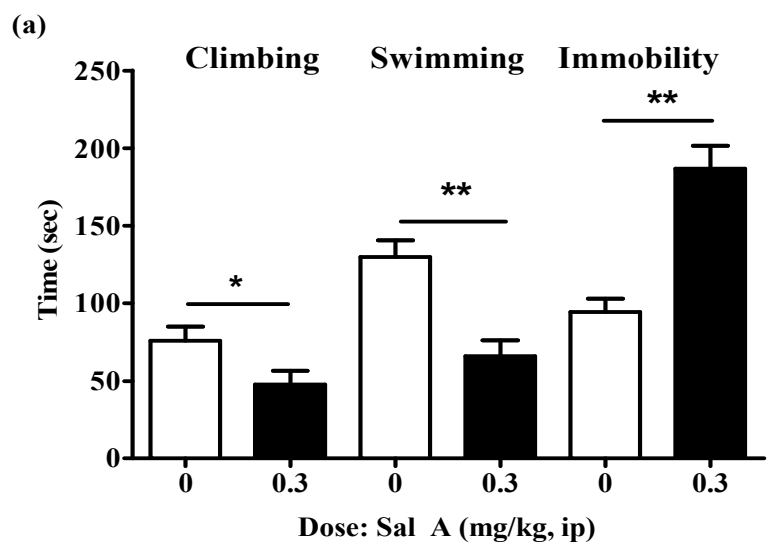

(b)

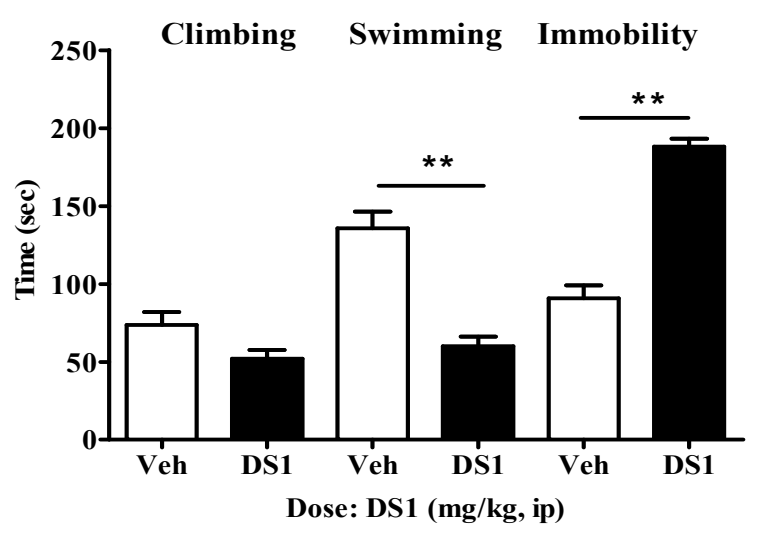

(c)

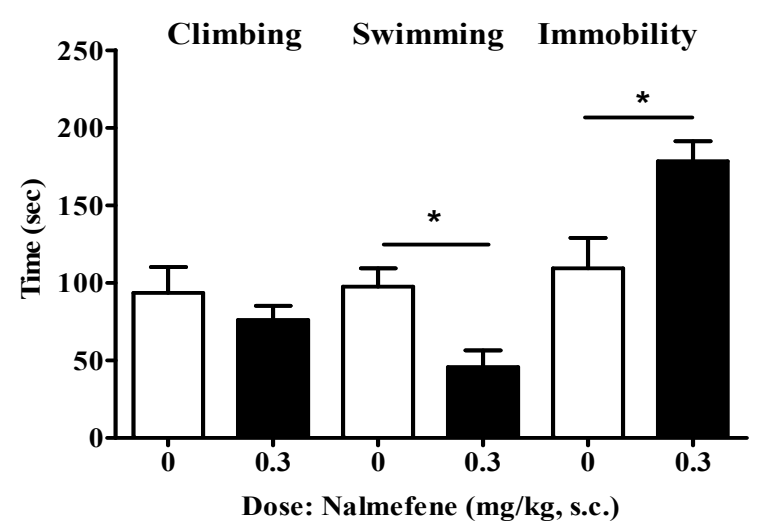

\section{Fig. 3.7. Effect of KOPr compounds on FST in drug naive rats.}

Drug naive animals were habituated to the forced swimming sessions on day 1 . The following day, animals received (a). Sal A (0, $0.3 \mathrm{mg} / \mathrm{kg} ; \mathrm{n}=6)$, (b). DS1 (0, $0.3 \mathrm{mg} / \mathrm{kg} ; n=5-7)$ and (c). nalmefene (0, $0.3 \mathrm{mg} / \mathrm{kg} ; \mathrm{n}=6)$ and were subjected to FST. The forced swimming behaviours were recorded for a period of $5 \mathrm{~min}$ and analysed in a bin of $5 \mathrm{sec}$ intervals. Data expressed as mean time in sec (+SEM) spent by animals for climbing, swimming and immobile behaviours during FST. ${ }^{*} p<0.05$, **p<0.01. Data for $0.3 \mathrm{mg} / \mathrm{kg}$ compared with $0 \mathrm{mg} / \mathrm{kg}$ for corresponding climbing, swimming and immobile behaviours. Mann Whitney test. 


\subsubsection{Effect of SERT blockade by FLX on KOPr agonists induced immobility in FST.}

The purpose of this test was to determine the role of SERT blockade via subchronic FLX pre-treatment on different forced swimming behaviours produced by Sal A and DS1 exposure in rats. Statistical analysis shows that FLX pre-treatment significantly reduced the amount of time spent immobile in animals pre-treated with Sal $\mathrm{A}[\mathrm{F}(1,17)=4.95, \mathrm{P}<0.05 ;$ Fig 3.8a $]$ or $\mathrm{DS} 1[\mathrm{~F}(1,17)=7.25, \mathrm{P}=0.015 ;$ Fig 3.8b $]$. A significant increase in the amount of time spent climbing was also noted in Sal A [F $(1,17)=8.01, \mathrm{P}=0.012$; Fig 3.8a $]$ and $\mathrm{DS} 1[\mathrm{~F}(1,17)=5.44, \mathrm{P}<0.05$; Fig 3.8b] pretreated animals, following FLX pre-treatment. No effect of FLX pre-treatment on swimming time was observed in Sal A $[\mathrm{F}(1,17)=0.15, \mathrm{P}=0.7$; Fig 3.8a] or DS1 [F (1, $17)=1.75, \mathrm{P}=0.2 ;$ Fig $3.8 \mathrm{~b}]$ treated rats. 
(a)

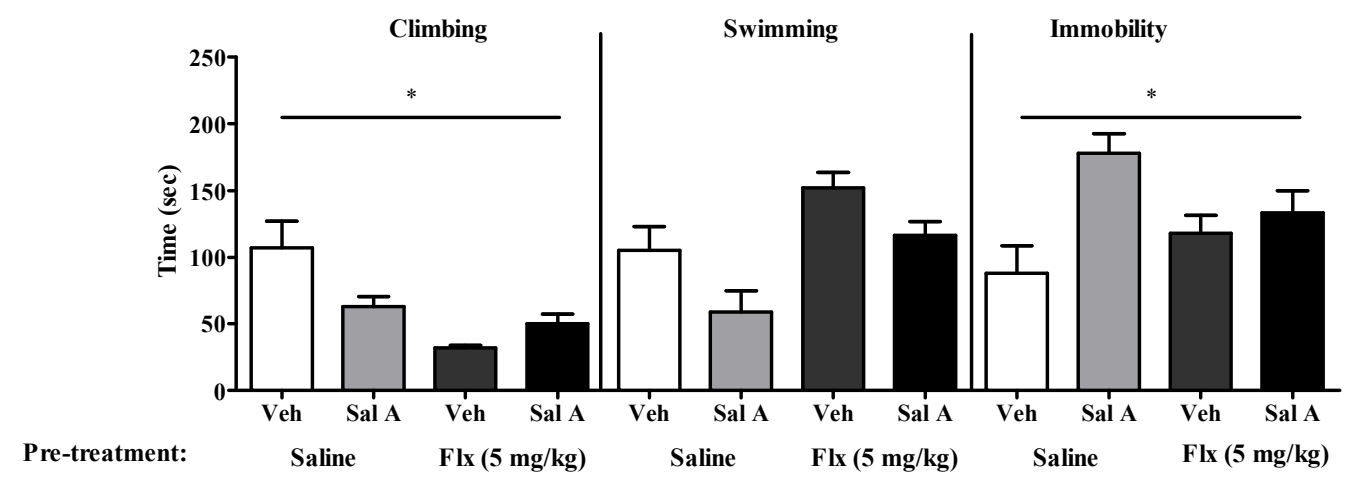

(b)

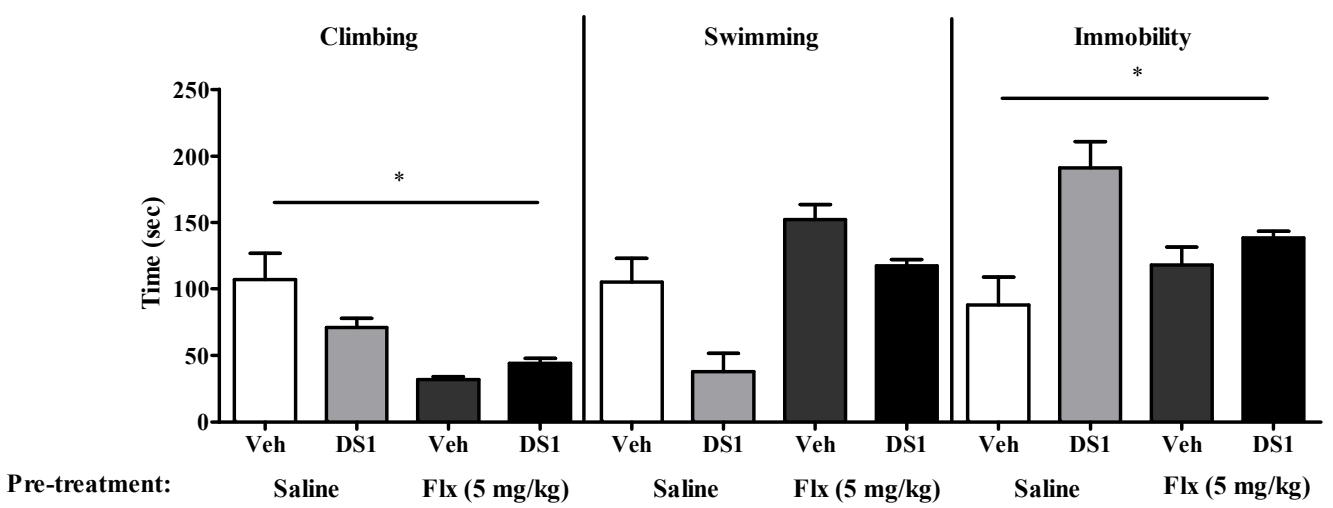

Fig. 3.8. Effect of SERT blockade by FLX pre-treatment on KOPr agonist induced immobility in FST.

Drug naive rats were habituated to the forced swimming test on day 1. Rats received injections of FLX (0, $0.3 \mathrm{mg} / \mathrm{kg}, \mathrm{sc}), 30 \mathrm{~min}, 19 \mathrm{hr}$ and $23 \mathrm{hr}$ post habituation sessions. The next day (24 hr following habituation sessions), animals received an injection of a) Sal A (0, $0.3 \mathrm{mg} / \mathrm{kg})$ or b) DSI (0, $0.3 \mathrm{mg} / \mathrm{kg}$ ) and were subjected to FST. The forced swimming behaviours were recorded for a period of 5 min and analysed in a bin of 5 sec intervals. Data expressed as mean time in sec (+SEM) for climbing, swimming or immobile during FST. ${ }^{*} p<0.05$, two-way ANOVA for each FST behaviours. $n=5-6$ for each group. 


\subsubsection{Control experiments for ASP ${ }^{+}$uptake}

Control experiments were performed to show a correlation between the level of $\mathrm{ASP}^{+}$accumulation and GFP-hSERT expression levels. An image of GFP-hSERT fluorescence was captured, followed by the addition of $\mathrm{ASP}^{+}$at time 0 . The $\mathrm{ASP}^{+}$ accumulation was measured every $5 \mathrm{sec}$ for $5 \mathrm{~min}$. Statistical analysis showed a linear correlation between GFP-hSERT expression and $\mathrm{ASP}^{+}$accumulation in transfected cells $\left(r^{2}=0.82\right.$; Goodness of fit, $p<0.0001$; Spearman coefficient, $n=80$; Fig. 3.9a).

The amount of $\mathrm{ASP}^{+}$accumulation in cells not expressing GFP-hSERT (nontransfected cells) was compared with those cells expressing GFP-hSERT (transfected cells). Our results showed a significant increase in $\mathrm{ASP}^{+}$accumulation in transfected cells vs. non-transfected cells $\left(\mathrm{p}<0.0001\right.$; Fig. 3.9b), thus indicating increased $\mathrm{ASP}^{+}$ uptake in cells expressing GFP-hSERT. 


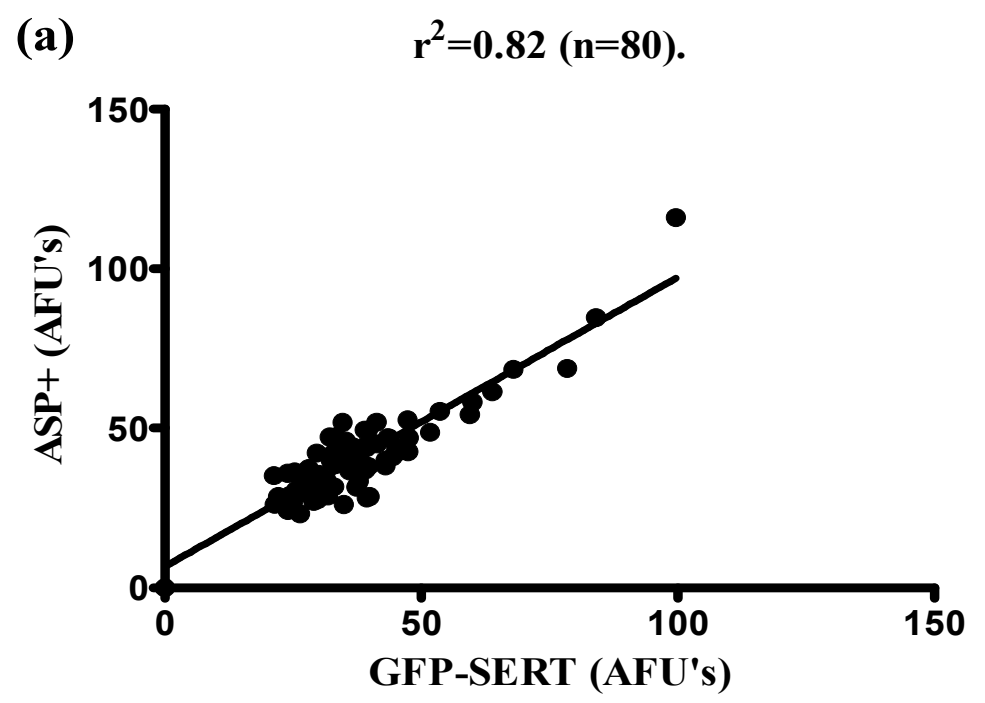

(b)

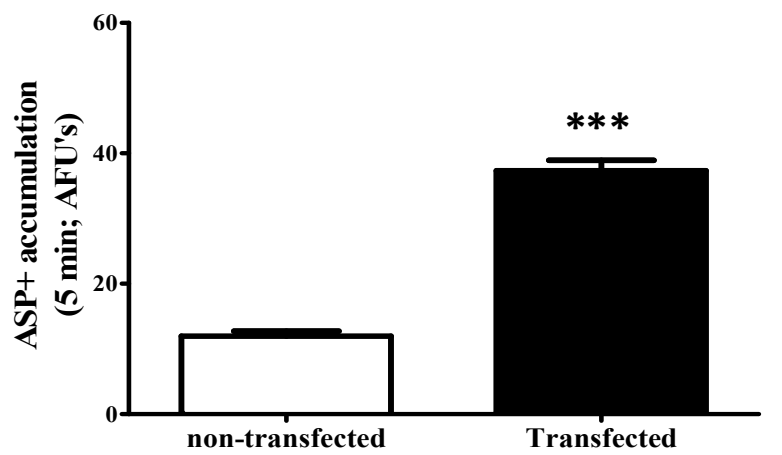

\section{Fig. 3.9. Control experiments for ASP ${ }^{+}$uptake.}

a) ASP ${ }^{+}$uptake and GFP-hSERT expression levels. GFP-hSERT levels for each cell are shown at time 0 (x axis). The accumulation of $A S P^{+}$after 5 min is shown for each cell on the $y$ axis. $A S P^{+}$and GFP-hSERT fluorescence are measured as arbitrary fluorescence units (AFU). Each dot indicates GFP-hSERT expression levels and corresponding $A S P^{+}$accumulated at 5 min by that particular cell. Data were analysed using linear regression. $n=80$. b) $A S P^{+}$accumulation in transfected vs. non-transfected cells. Bars indicate the amount of $\mathrm{ASP}^{+}$accumulated in transfected and non-transfected cells and measured as AFU. ${ }^{* * *} p<0.0001$, data compared with a non-transfected group of cells using student t-test. Number of non-transfected cells $=24$, transfected cells $=80$. 


\subsubsection{Effect of KOPr agonists pre-treatment on ASP ${ }^{+}$uptake}

The effect of KOPr agonist pre-treatment on GFP-hSERT function in GFPhSERT and myc-rKOPr co-transfected HEK-293 cells using the $\mathrm{ASP}^{+}$uptake method is shown in Fig. 3.10a. Cultures were pre-treated with $10 \mu \mathrm{M}$ U50488H, Sal A or DS1 and following a $30 \mathrm{~min}$ incubation, SERT function was measured. Pre-treatment with the traditional KOPr agonist U50488H significantly decreased $\mathrm{ASP}^{+}$uptake $(\mathrm{p}<0.001)[\mathrm{F}$ $(3,261)=106.1, \mathrm{p}<0.0001]$. However, there was no significant difference in $\mathrm{ASP}^{+}$ accumulation in cells pre-treated with either Sal A or its structural analogue DS1 $(\mathrm{p}>0.05)$, thus indicating a potential difference in modulation of SERT function by novel and traditional KOPr agonists.

In order to verify that changes seen in $\mathrm{ASP}^{+}$uptake were specifically due to KOPr agonist treatment and not due to selection of cells with lower levels of GFPhSERT expression levels, GFP-hSERT fluorescence intensity for cells pre-treated with $\mathrm{KOPr}$ agonists and selected for $\mathrm{ASP}^{+}$accumulation analysis were compared with vehicle treated cells. Statistical analysis revealed no significant difference in GFPhSERT expression levels in cells analysed for $\mathrm{ASP}^{+}$uptake $[\mathrm{F}(3,261)=2, \mathrm{p}=0.11$; Fig. $3.10 \mathrm{~b}]$. 
(a)

5 min uptake

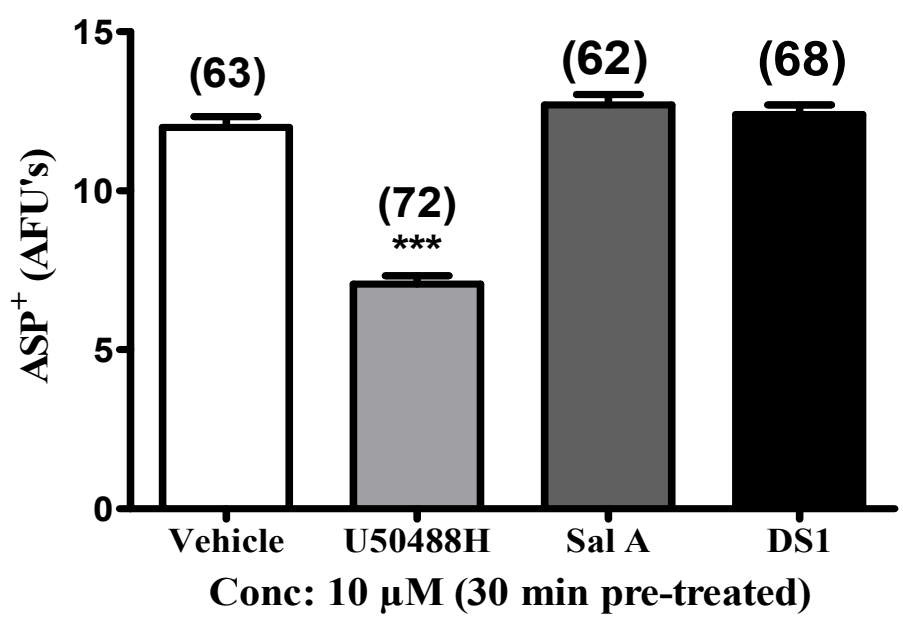

(b)

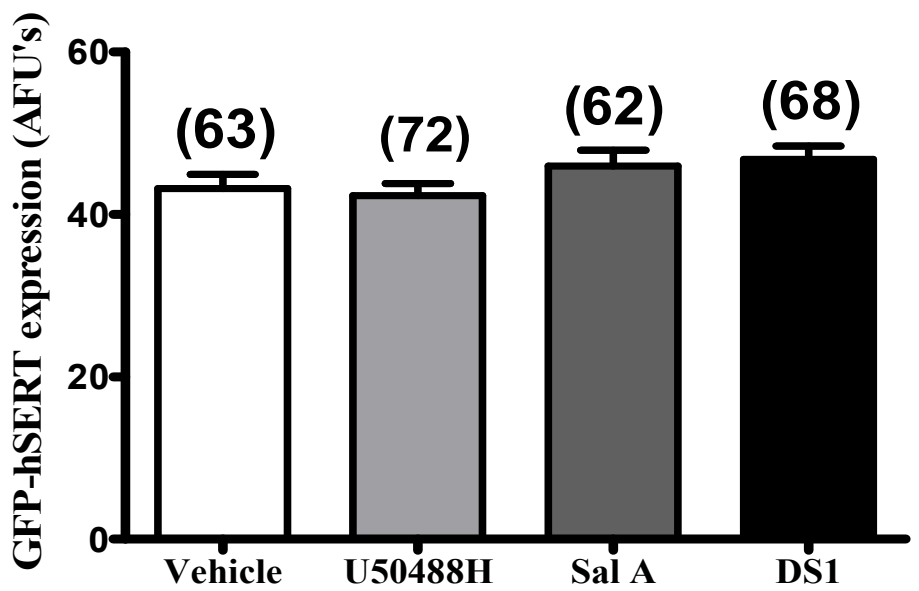

\section{Fig. 3.10. Effect of KOPr agonists pre-treatment on $A S P^{+}$uptake}

a) HEK-293 cells co-transfected with GFP-hSERT and myc-rKOPr were pre-treated with KOPr agonists (U50488H, Sal A and DS1; $10 \mu \mathrm{M}$ each) for $30 \mathrm{~min}$ and ASP ${ }^{+}$ accumulation was recorded for $5 \mathrm{~min}$. The quantification of $A S P^{+}$fluorescence was measured as arbitrary fluorescence units (AFU). Figures indicates Mean (+SEM) AFU's. ${ }^{* * *} P<0.001$, one-way ANOVA followed by Dunnet multiple comparison test. $b$ ) Symbols indicate the amount of GFP-hSERT expression measured in AFU's for cells selected for ASP ${ }^{+}$uptake analysis. One-way ANOVA followed by Dunnet multiple comparison tests. Numbers in parentheses indicate sample size. 
3.4. Summary of results.

$\underline{\text { Tab. 3.2. Control experiments to determine selectivity of KOPr agonists in }}$

\section{attenuating reinstatement}

\begin{tabular}{|c|c|c|}
\hline $\begin{array}{c}\text { Compounds } \\
\text { tested }\end{array}$ & $\begin{array}{c}\text { Cocaine induced hyperactivity in } \\
\text { self-administering rats (motor } \\
\text { suppression during reinstatement } \\
\text { test) }\end{array}$ & $\begin{array}{c}\text { Sucrose reinforcement } \\
\text { test (effect on operant } \\
\text { responding for natural } \\
\text { reward) }\end{array}$ \\
\hline Sal A & No effect & No effect \\
\hline DS1 & Non-significant trend to decrease & No effect \\
\hline MOM Sal B & No effect & Significant attenuation \\
\hline Nalmefene & Non-significant trend to increase & No effect \\
\hline
\end{tabular}

\section{Tab. 3.3. Adverse effect profile of novel KOPr agonists}

\begin{tabular}{|c|c|c|c|}
\hline $\begin{array}{c}\text { Compounds } \\
\text { tested }\end{array}$ & $\begin{array}{c}\text { Motor function } \\
\text { (spontaneous open field } \\
\text { activity) }\end{array}$ & $\begin{array}{c}\text { Aversion } \\
\text { (CTA) }\end{array}$ & $\begin{array}{c}\text { Depressive } \\
\text { behaviours (FST) } \\
\text { (Cocaine exposed and } \\
\text { drug naive rats) }\end{array}$ \\
\hline Sal A & No effect & No effect & Inceased immobility \\
\hline DS1 & No effect & No effect & Inceased immobility \\
\hline MOM Sal B & No effect & - & - \\
\hline Nalmefene & Significant suppression & No effect & Inceased immobility \\
\hline
\end{tabular}

\section{Tab. 3.4. Effect of FLX pre-treatment on KOPr agonist induced enhanced immobility in FST}

\begin{tabular}{|c|c|}
\hline Compound tested & Effect on depressive behaviour \\
\hline Sal A & Improved \\
\hline DS1 & Improved \\
\hline
\end{tabular}

Tab. 3.5. Effect on SERT modulation by traditional and novel KOPr agonists by using live cell ASP ${ }^{+}$uptake.

\begin{tabular}{|c|c|}
\hline Compound tested & ${\text { Effect on } \text { ASP }^{+} \text {uptake }}^{\text {U50488H }}$ \\
\hline Sal A & Decreased \\
\hline DS1 & No effect \\
\hline
\end{tabular}




\subsection{Discussion}

In Chapter 2 we showed that a single injection of either Sal A, DS1, MOM Sal B or nalmefene attenuated cocaine prime induced reinstatement. In the present chapter we evaluated whether this attenuation in cocaine seeking behaviour was selective to reinstatement. We tested this by measuring cocaine induced hyperactivity (in cocaine self-administering rats) and disruption of responding for palatable reinforcements (sucrose reinforcement). We also performed studies to determine if side effects such as sedation, aversion and depressive like behaviour resulted following acute administration of novel KOPr agonists. Initially FST experiments were performed using animals with history of cocaine self-administration (Tab. 3.1). These pilot studies were necessary to develop this paradigm in our laboratory and to obtain permission from the Animal Ethics Committee to test the effect of KOPr compounds (Sal A, DS1, nalmefene) in drug naive rats. In order to determine a possible mechanism for the depressive like effects of KOPr agonist treatment, the effect of blocking SERT on KOPr agonists induced depressive behaviours was tested using FLX in rats. Additionally, the effect of SERT modulation by KOPr agonists was tested in vitro by using live cell $\mathrm{ASP}^{+}$uptake assay.

The results from the present study indicate that acute exposure to Sal A $(0.3$ $\mathrm{mg} / \mathrm{kg})$, DS1 (0.3 mg/kg; Fig 3.2a), MOM Sal B (0.3 mg/kg; Fig 3.2c) or nalmefene (0.3 mg/kg; Fig. 3.2d) did not produce any statistically significant motor suppression during the reinstatement test. However, on closer inspection, DS1 $(0.3 \mathrm{mg} / \mathrm{kg})$ exposure showed a non-significant trend towards a decrease in motor function (Fig 3.2b). Results from Chapter 2 indicate that DS1 pre-treatment significantly attenuated acute cocaineinduced hyperactivity in rats (Tab 2.4). The observed difference in DS1's ability to modulate cocaine-induced locomotion might be attributed to the multiple cocaine exposures as animals used in this study previously self-administered cocaine (Section 
3.2.5.1). Drug naive animals were used for experiments outlined in Chapter 2 (Section 2.2.4.2 A). Acute nalmefene injections, on the other hand, showed a non-significant trend towards an increase in motor function (Fig 3.2d). Acute exposure to Sal A, DS1 or nalmefene, at doses that successfully attenuated cocaine seeking, did not suppress sucrose reinforcement (Fig. 3.3). In contrast, MOM Sal B significantly suppressed sucrose reinforcements (Fig. 3.3c). These results indicate that Sal A (Morani et al., 2009) and an analogue with similar potency at KOPr (DS1), attenuated cocaine prime induced drug-seeking behaviour without reducing sucrose reinforcements. This however cannot be said for the more potent and longer acting Sal A analogue, MOM Sal B (Tab 3.2).

Previous studies by June and colleagues (1998), showed that nalmefene pretreatment attenuated ethanol-maintained operant lever press responding without affecting saccharin reinforcement. Their study also showed that the efficacy of nalmefene was dependent on the route of administration ( $\mathrm{sc}>$ oral route) and pretreatment time (15 min prior to the commencement of tests). Injections of nalmefene bilaterally into the NAc $(1.0,10 \mu \mathrm{g})$ and VTA $(10 \mu \mathrm{g})$ also attenuated ethanol induced responding without affecting saccharin reinforcement (June et al., 2003). However, infusing a higher dose $(40 \mu \mathrm{g})$ of nalmefene bilaterally into both the VTA and NAc suppressed ethanol and saccharin reinforcement. These findings indicate a dose related effect produced by nalmefene in selectively attenuating alcohol self-administration (June et al., 2003). Since the dose of nalmefene tested in this current study $(0.3 \mathrm{mg} / \mathrm{kg})$ attenuated cocaine induced reinstatement without suppressing sucrose reinforcements, this effect produced by nalmefene on cocaine seeking behaviour (Chapter 2) was deemed to be selective for cocaine (Tab 3.2).

The effects of a single injection of Sal A $(0.3 \mathrm{mg} / \mathrm{kg})$, DS1 $(0.3 \mathrm{mg} / \mathrm{kg}), \mathrm{MOM}$ Sal B $(0.3 \mathrm{mg} / \mathrm{kg})$ and nalmefene $(0.3 \mathrm{mg} / \mathrm{kg})$ on spontaneous open field activity in rats were determined. Our results indicate that acute injections of Sal A, MOM Sal B and 
DS1 did not suppress spontaneous open field activity in rats (Fig. 3.4). These results show that attenuation of both cocaine induced behavioural sensitization by Sal A and DS1 (Chapter 2) and cocaine induced hyperactivity by DS1 in drug naive rats (Chapter 2) was a not due to motor suppression caused by Sal A and DS1 pre-treatment. Furthermore, previous reports have shown that acute exposure to Sal A did not suppress open field activity in rats (Hooker et al., 2009a; Carlezon et al., 2006). Similarly, MOM Sal B exposure did not produce motor suppression in rats (Wang et al., 2008). These published reports support our findings in the current study.

A single injection of nalmefene, however significantly suppressed motor function in the drug naive rats (Fig. 3.4d). Since, nalmefene pre-treatment did not decrease cocaine induced hyperactivity during reinstatement tests, an observable difference in motor function induced by nalmefene exposure might be attributed to the prior cocaine exposure in self-administering rats (Tab. 3.3). Additionally, as all compounds tested having selective affinity for KOPr (Sal A, DS1, MOM Sal B) did not produce locomotor suppression; a possible role of MOPr antagonism might be implicated in nalmefene produced motor suppression in drug naive rats. Further studies are required to explain this. On close observation, animals treated with 75\% DMSO (Fig $3.4 \mathrm{a}, 3.4 \mathrm{~b}, 3.4 \mathrm{c})$ showed less locomotor activity compared to rats treated with $0.9 \%$ saline (Fig 3.4d). As nalmefene treated animals produced approximately similar ambulatory counts compared to the other KOPr compounds tested (Sal A, DS1, MOM Sal B, Fig 3.4); the suppression in motor function produced by nalmefene in the spontaneous locomotion test may reflect a floor effect.

In order to evaluate the aversive behaviours produced by acute exposure to novel KOPr agonists, a conditioned taste aversion model was used. Our results indicated that Sal A $(0.3 \mathrm{mg} / \mathrm{kg})$ and nalmefene $(0.3 \mathrm{mg} / \mathrm{kg})$ when paired with the novel tasting saccharin solution, did not induce aversive effects in rats (Fig. 3.5a, 3.5c; Tab. 3.3). 
However, Sal A treated rats showed a non-significant trend to increase saccharin drinking on the test day compared to pairing day (Fig 3.5a). Rats paired with DS1 showed a significant increase in saccharin consumption on test day when compared to pairing day (Fig 3.5b; Tab 3.3). Previous reports suggest that Sal A at low doses produce a preference for conditioned behaviours in rats and zebrafish (Braida et al., 2007; 2008). The preference for a novel tasting saccharin solution produced by low dose of Sal A $(0.3 \mathrm{mg} / \mathrm{kg})$ and DS1 $(0.3 \mathrm{mg} / \mathrm{kg})$ may be a dose related effect. One aim of this study was to determine if the dose of KOPr agonist that successfuly attenuated drug-seeking had aversive properties. We measured this by using a taste aversion model and showed that acute exposure to all three KOPr compounds did not produce CTA (Tab 3.3). However, detailed studies are required to compare and contrast the aversive properties of different dose of Sal A, DS1 and nalmefene with standard aversive agent such as lithium chloride (Taraschenko et al., 2010). Such studies have the potential to further characterize the aversive properties of these KOPr compounds.

Recent studies have shown that systemic administration of Sal A $(2.0 \mathrm{mg} / \mathrm{kg})$ increases ICSS threshold in rats and decreases phasic DA release in the NAc (Ebnar et al., 2010). This same study also showed that Sal A (2 mg/kg) also significantly decreased sucrose reinforcements using both FR-5 schedule and progressive ratios schedule of reinforcement (Ebnar et al., 2010). In contrast, a lower dose of Sal A (0.25 $\mathrm{mg} / \mathrm{kg}$ ) did not modulate ICSS thresholds, alter sucrose reinforcement or decrease phasic DA release in NAc (Ebnar et al., 2010). Moreover, results from our current study indicate that Sal A at comparatively lower doses $(0.3$ and $1.0 \mathrm{mg} / \mathrm{kg})$ did not suppress sucrose reinforcement using a FR-5 schedule of reinforcement (Morani et al., 2009). The ip route of administration for Sal A and the vehicle control (75\% DMSO) in this study were comparable to those used by Ebnar et al, (2010), indicating that Sal A induced aversive effects might be dose related. Similarly, Sal A at higher dose produced 
place aversion in C57BL/6J mice (1.0, $3.2 \mathrm{mg} / \mathrm{kg}$; Zhang et al., 2005), zebrafish (80 $\mu \mathrm{g} / \mathrm{kg}$; Braida et al., 2007) and Wistar rats (160 $\mu \mathrm{g} / \mathrm{kg}$; Braida et al., 2008). Therefore, Sal A $(0.3 \mathrm{mg} / \mathrm{kg})$ produces anti-cocaine effects without producing aversion, motor suppression or anhedonia in rats (Morani et al., 2009).

Our initial studies investigating the depressive like effects produced by KOPr compounds were performed on rats with a previous history of cocaine selfadministration. These results showed that Sal A $(0.3 \mathrm{mg} / \mathrm{kg}), \mathrm{DS} 1(0.3 \mathrm{mg} / \mathrm{kg})$ and nalmefene $(0.3 \mathrm{mg} / \mathrm{kg})$ significantly increased immobility time in the FST (Fig. 3.6). These preliminary findings led to the subsequent FST studies in drug naive rats, which showed that all the KOPr compounds tested also produced pro-depressive behaviours (Fig. 3.7; Tab. 3.3).

Depression induced by Sal A and DS1 might not be due to suppression in motor function, as single exposure to either compound $(0.3 \mathrm{mg} / \mathrm{kg})$ had no effect on spontaneous open field activity (Tab 3.3). However, treatment with nalmefene (0.3 $\mathrm{mg} / \mathrm{kg}$ ) induced sedation, as shown by the results from spontaneous locomotion test (Fig 3.4d). Therefore, the depressive effects produced by nalmefene in drug naive rats might not be specific. However, nalmefene has been shown to stimulate the hypothalamic pituitary adrenal axis, which might explain the depressive effects observed in this current study (Geer et al., 2005). Since depression is one of the limiting factors in developing selective KOPr agonists as anti-addiction pharmacotherapies, future studies with nalmefene may help to address wether this behavioural profile is more likely induced by mixed KOPr ligands compared to selective KOPr agonists.

Previous reports have shown that Sal A produces both anti-depressant (Braida et al., 2008; 2009) and pro-depressive effects (Carlezon et al., 2006) in rats using the FST paradigm. The present study determined whether an acute injection of Sal A $(0.3 \mathrm{mg} / \mathrm{kg})$ 
at a dose which attenuated cocaine seeking (Chapter 2) produced depressive effects or not. The results from this study suggest that acute exposure to Sal A $(0.3 \mathrm{mg} / \mathrm{kg})$ produces pro-depressive behaviour without suppressing spontaneous locomotion (Fig. 3.7a; Fig. 3.3a). These results are in agreement with the findings of Carlezon et al., (2006) which showed that pre-treating rats with Sal A, dose dependently $(0.25-2.0$ $\mathrm{mg} / \mathrm{kg}$ ) produced pro-depressive effects without suppressing their open field locomotion activity. The difference in the experimental design of this study was that Carlezon and colleagues, (2006) had pre-treated rats with Sal A, thrice, at $30 \mathrm{~min}, 19 \mathrm{hr}$ and $23 \mathrm{hr}$ post habituation, whereas, in our study, animals received a single injection of Sal A, 5 min prior to the FST. In contrast to these findings, Braida et al., (2009) showed that acute Sal A up to $1 \mathrm{mg} / \mathrm{kg}$ produced anti-depressant effects in rats using FST paradigm. The observable dissimilarities may be attributed to the difference in; route of administration for Sal A (i.p. current study, Carlezon et al., 2006 vs. s.c. Braida et al., 2009); time of Sal A pre-treatment (5 min, current study vs. 20 min Braida et al., 2009), and vehicle used to suspend Sal A (75\% DMSO, current study, Carlezon et al., 2006 vs. 1:1:8, Ethanol: Tween 80: Water, Braida et al., 2009).

From the present findings, it was interesting to note that in drug naive rats, Sal A significantly decreased climbing time and swimming time, whereas DS1 significantly reduced time spent swimming, although a trend to decrease climbing behaviour was also noted (Fig 3.7a, 3.7b). In contrast nalmefene only decreased swimming time without any effect on climbing behaviour. Carlezon et al. (2006) also showed that Sal A decreased swimming time during the FST. A decrease in climbing time is believed to relate to NET modulation, whereas SERT is implicated in a reduction in swimming time (Detke et al., 1995). Taken together, these findings implicate a possible involvement of SERT and NET in depressive-like behaviours induced by Sal A and DS1 in the FST and only SERT in nalmefene induced depression. Further studies are required to clarify this. 
In order to determine if Sal A and DS1 modulate depressive behaviour via SERT modulation, we measured depressive behaviours following SERT antagonist (FLX) pretreatment followed by Sal A, DS1 or vehicle. Previous studies have shown that pretreating rats with $\operatorname{FLX}(5,10,20 \mathrm{mg} / \mathrm{kg}, \mathrm{sc}), 30 \mathrm{~min}, 19 \mathrm{hr}$ and $23 \mathrm{hr}$ post FST habituation induces anti-depressant effects using the FST model (Detke et al., 1995). In this present study, a low dose of FLX $(5 \mathrm{mg} / \mathrm{kg})$ that has been shown previously to have anti-depressant effects in the FST was used (Detke et al., 1995). The results from our study show that pre-treating rats with the minimum effective dose of FLX significantly improved Sal A (Fig 3.8a) and DS1 (Fig 3.8b) induced depressive behaviours. This was noted by a reduction in immobility times and enhancement in time spent climbing with both Sal A and DS1. Additionally, a non-significant increase in swimming time was observed for saline/Sal A vs. FLX/Sal A and saline/DS1 vs FLX/DS1 treated animals (Fig 3.8). Thus sub-chronic FLX treatment reduced depressive behaviours produced by KOPr agonists (Tab. 3.4).

Exposure to FLX (1- $20 \mathrm{mg} / \mathrm{kg})$ dose dependently increases 5HT levels in raphae nucleus, ventral hippocampus and frontal cortex when measured using in vivo microdialysis (Malagie et al., 1995). As mentioned earlier, KOPr activation has been shown to decrease 5HT levels in brain regions implicated in depressive behaviours (Tao and Auerbach, 2005; Yilmaz et al., 2006). Therefore, modulating 5HT levels by FLX pre-exposure might be responsible for the observable improvement in KOPr agonist induced depression in FST. An increase in phosphorylated CREB levels in the NAc shell has been implicated in depressive like behaviours in the FST (Plaikas et al., 2001). Moreover, KOPr activation increases CREB phosphorylation in NAc, which is antagonised by FLX, desimipramine (NET inhibitor) and KOPr antagonists (Carlezon et al., 1998; Mague et al., 2003; Chartoff et al., 2009). Therefore, decreased CREB activation by FLX pre-treatment may be responsible for diminishing KOPr agonists' 
induced depressive-like behaviours. Detailed studies on this will help us to better understand the mechanisms underlying depressive effects produced by novel KOPr agonists.

The model of FST described by Porsolt et al., (1979) has been used to pharmacologically evaluate anti-depressants (Castagne et al., 2010). In 1995, Detke and colleagues modified the FST apparatus and analysis of the forced swimming behaviours to enhance the efficiency of the paradigm to evaluate anti-depressants (see Detke et al., 1995). As FST has been previously been applied to measure depressive effects produced by KOPr agonists (Pliakas et al., 2001; Mague et al., 2003; Carlezon et al., 2006), we also used this model to measure KOPr agonist induced behavioural despair in this current study. However, the use of FST as a model to evaluate behavioural despair has its limitations (Borsini and Meli, 1988; Castagne et al., 2010). The major limitation with FST is that the immobility behaviour might be due to the animal getting adjusted to the swimming conditions rather than actual behavioural despair, which might lead to false result interpretation (Borsini and Meli, 1988; Castagne et al., 2010). Therefore future studies with Sal A, DS1 and nalmefene are needed to evaluate their effects in inducing anhedonia and behavioural despair. Such experiments have been mentioned in detail in Chapter 4.

Due to the behavioural results with FLX to improve Sal A and DS1 induced depressive behaviours in FST, we conducted preliminary experiments to determine if KOPr directly modulates SERT protein. To do this we used an in vitro cell model. Cells were treated to transiently express GFP-hSERT and myc-rKOPr, followed by treatment with traditional (U50488H) and novel (Sal A and DS1) KOPr agonist's, and SERT function was measured in real-time.

Control experiments were performed to validate that $\mathrm{ASP}^{+}$uptake is dependent on the level of GFP-hSERT expression in single cells (Fig. 3.9a). The amount of ASP ${ }^{+}$ 
accumulated by non-transfected cells was significantly lower than the uptake shown by transfected cells (Fig. 3.9b). These findings are in accordance with the recently published findings by $\mathrm{Oz}$ et al., (2010). The results from the current study also indicate that a 30 min pre-incubation with the novel KOPr agonists Sal A $(10 \mu \mathrm{M})$ and DS1 (10 $\mu \mathrm{M})$ did not have any significant effect on modulating SERT function using the ASP ${ }^{+}$ accumulation assay in single cells (Fig. 3.10a). However, pre-incubating cells with the classic KOPr agonist, U50488H (10 $\mu \mathrm{M}$ for $30 \mathrm{~min})$ significantly attenuated $\mathrm{ASP}^{+}$ uptake (Fig. 3.10a). This effect produced by the KOPr agonist was a SERT-selective effect as $\mathrm{ASP}^{+}$accumulation was dependent on SERT expression levels (Fig. 3.9a) and no significant difference in GFP-hSERT expression levels were observed in cells selected from each treatment group (Fig. 3.10b). This, to the best of our knowledge, is the first study to determine the effect of KOPr agonist in modulating SERT function in vitro. However, as acute KOPr agonists show anti-addiction properties (Chapter 2) with depression as an adverse effect (Chapter 3), understanding the effect of acute exposure to KOPr agonists on modulating SERT function is necessary to confirm and quantify the findings from the present study. Also, such study has the potential to flesh out the role of SERT modulation in KOPr agonists produced depression.

In this study, we captured the image of the whole cell SERT which included the amount of SERT expressed on the cell surface and within the cell. Also, the initial SERT measurements were recorded after KOPr compounds/vehicle incubations. The $\mathrm{ASP}^{+}$uptake has been shown to be mediated by the SERT expression levels on the cell surface (Oz et al., 2010; Schwartz et al., 2003). Thus, in this present experiment we cannot measure the effect on $\mathrm{ASP}^{+}$accumulation due to transporter internalization which might have occurred due to KOPr agonist pre-treatment. In order to quantify the amount of $\mathrm{ASP}^{+}$accumulation due to SERT internalization, further imaging experiments are required. Such studies can help in differentiating the effect of chronic 
vs. acute KOPr activation on modulating cell surface SERT expression and transporter internalization.

Previous studies have shown that Sal A has a greater affinity for KOPr than U50488H and U69593 and equal affinity compared to DYN (Chavkin et al., 2004). Furthermore, Sal A pre-treatment has been shown to produce 40 times less KOPr internalization than U50488H (Wang et al., 2005), whereas, another study indicated Sal A and $\mathrm{U} 50488 \mathrm{H}$ produced internalization to a similar extent (Wang et al., 2008). Since, in this experiment, the KOPr agonists were pre-incubated for $30 \mathrm{~min}$, KOPr may have been internalised after treatment with $\mathrm{U} 50488 \mathrm{H}$ which may not have occurred with Sal A or DS1. Therefore, further studies are required to compare and contrast the effect of traditional and novel Sal A like KOPr agonists on KOPr internalization. Since acute KOPr activation has been shown to produce anti-addiction behaviours in pre-clinical models, it would be interesting to note the effects of acute activation of KOPr on SERT function and kinetics. This model ( $\mathrm{ASP}^{+}$uptake) can be used in future to study the effect of SERT modulation following acute exposure to novel and classical KOPr agonists. Such experiments may aid in the development of KOPr analogues that vary in their effects on SERT function.

\subsection{Summary and Conclusion}

The results from sucrose-reinforced responding showed that Sal A, DS1 and nalmefene attenuated cocaine seeking behaviour in a selective manner (Chapter 2), whereas MOM Sal B attenuated cocaine seeking in a non-selective manner. Sal A and DS1 did not affect motor function or produce aversion, while nalmefene suppressed motor function without producing aversion in cocaine naive rats. All three KOPr agonists induced depressive behaviours. Furthermore, we provide preliminary evidence to suggest that the Sal A and DS1 induced depressive behaviours may involve negative modulation of 5HT systems which was attenuated by SERT antagonist. Further in vitro 
studies exploring SERT modulation by KOPr agonists indicated a potential difference in modulation of SERT function by classical KOPr agonist U50488H and novel neoclerodane diterpenes Sal A and DS1. 
Chapter 4. General Discussion 


\subsection{Endogenous KOPr systems and cocaine addiction}

As mentioned earlier (Chapter 1, Section 1.5), central KOPr are up-regulated by repeated cocaine exposures (Collins et al., 2002). During this initial hedonic stage of cocaine use, there is an increased sensitization of the midbrain dopamine system which has been shown to be attenuated by KOPr activation (Glick et al., 1996; Archer et al., 1996). Also, studies by Chefer and colleagues (2005) have shown that KOPr gene deletion in mice increases DA levels and decreases DA re-uptake in the NAc. The same study also showed that KOPr knock-out mice remained in persistent cocaine sensitized state following a single cocaine injection (Chefer et al., 2005). Thus indicating that the KOPr systems might be implicated in controlling the dopaminergic effects produced by cocaine and activating KOPr could prevent the development and progression of cocaine addiction during the initial stages of the addiction cycle (Shippenberg et al., 2001; 2007; Mysels and Sullivan, 2009; Bruijnzeel, 2009; Fig 4.1).

Withdrawl from drug use has been associated with increased CREB phosphorylation in the NAc and the production of depressive like symptoms in laboratory animals (Nestler and Carlezon, 2001). KOPr activation has also been shown to enhance CREB phosphorylation in the NAc (Pliakas et al., 2001). A decrease in DA levels in the NAc during abstinence from the drug use has also been reported (Weiss et al., 1992). KOPr agonists have been shown to produce stress and depressive like symptoms in laboratory animals (Carlezon et al., 1998; 2006; Mague et al., 2003). On the other hand, KOPr antagonism enhances extracellular DA levels and decreases phosphorylated CREB levels in the NAc (Mague et al., 2003; Plaikas et al., 2001; Spanagel et al., 1990). KOPr antagonists also produce anti-depressant like effects in pre-clinical models (Spanagel et al., 1992; Mague et al., 2003; Nestler and Carlezon, 2006). Therefore, antagonizing KOPr signalling during the withdrawal stage of addiction cycle might be useful as replacement therapy to aid in preventing relapse to 
drug use (Shippenberg et al., 2007; Prisinzano, 2008). As there are no current pharmacotherapies available for cocaine addiction, manipulating the endogeneous KOPr systems might be a novel mechanism towards finding the treatment for cocaine addiction (Shippenberg et al., 2007; Mysels and Sullivan, 2009; Bruijnzeel, 2009; Fig 4.1). The present study addresses the first part of the problem, that is, developing safer KOPr agonists which could assist in breaking the addiction cycle with fewer KOPr agonists related adverse effects.

Reinstatement of extinguished cocaine self-administration by the presentation of stressors (foot-shock, forced swim stress; Erb et al., 1996) has been used pre-clinically to screen the anti-addictive potential of KOPr antagonists (Beardsley et al., 2005; 2010). Additionally, reinstatement of cocaine via stressors requires prior KOPr activation as KOPr knockout mice were insensitive to stress-produced reinstatement (Mclaughlin et al., 2003; Redila and Chavkin, 2008). KOPr antagonist administration does not modulate cocaine prime induced drug-seeking in laboratory rats (Beardsley et al., 2005). Furthermore, data from Chapter 2 shows that nor-BNI pre-treatment completely reversed Sal A (Fig 2.2b), DS1 (Fig 2.6b) and nalmefene (Fig 2.11b) produced attenuation of cocaine prime induced reinstatement. KOPr activation has also been shown to potentiate stress induced reinstatement in mice (Redila and Chavkin, 2008). Taken together, these findings suggest that the reinstatement of extinguished cocaineseeking initiated by a priming injection of cocaine is attenuated by KOPr activation. In contrast, blocking KOPr signalling attenuates reinstatement of extinguished cocaine seeking initiated by presentation of environmental stressors. This effect might reflect a possible difference in the central neuronal circuit involved in drug prime vs. stress induced reinstatement (Kreibich and Blendy, 2004) and activating or deactivating KOPr systems may modulate these behaviours in an opposing manner (Schenk et al., 1999; Redila and Chavkin, 2008; Beardsley et al., 2005). Given the fact that both these 
paradigms have been applied extensively to study cocaine reinstatement pre-clinically, they can also be used to evaluate the utility of KOPr agonists and antagonists as anticocaine agents. 


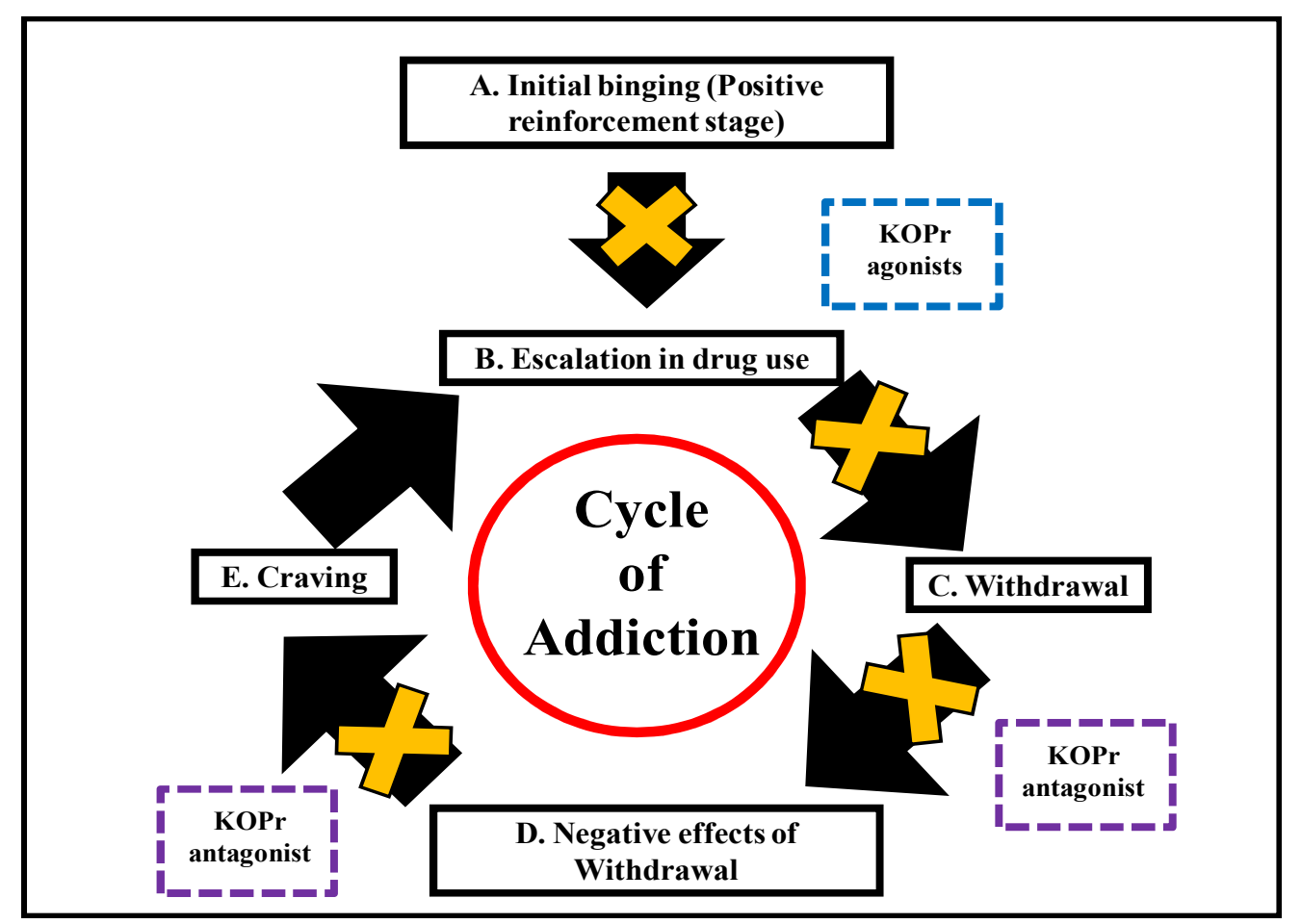

\section{Fig.4.1. KOPr system modulation in addiction cycle}

KOPr activation antagonises cocaine produced hedonic state and therefore could prove useful in managing the progression of the addiction cycle. KOPr antagonists, on the other hand produce anti-depressive effects and therefore antagonising KOPr systems could be useful in preventing relapse. 


\subsection{Limitations of classical KOPr agonists: rationale for Salvinorin A}

\section{screening.}

As described in Chapter 1 (section 1.5), the major limiting factor in developing synthetic KOPr agonists is the adverse effects associated with them (Prisinzano et al., 2005; Shippenberg et al., 2007; Wee and Koob, 2010). KOPr activation also produces dysphoria and psychotomimetic effects in humans (Walsh et al., 2001b; Pfeiffer et al., 1986). Salvia divinorum leaves have been used by the natives from Oaxaca, Mexico for the purpose of divination (Siebert et al., 1992; Ott, 1995; Valdes et al., 1994; Vorthermes and Roth, 2006). Also, its use as a recreational hallucinogen has recently gained widespread popularity among adolescents in the western countries (Vorthermes and Roth, 2006; Griffin et al., 2008; Kelly, 2011). With the discovery that Sal A produces its pharmacological effect via selectively binding and activating KOPr has opened new avenues in KOPr research (Roth et al., 2002; Prisinzano, 2005; Prisinzano et al., 2005; Pravett-Smith and Prisinzano, 2010). With this as a primary objective, several research groups have worked towards exploring the behavioural pharmacology of Sal A at KOPr (Carlezon et al., 2006; McCurdy et al., 2005; Fantagrossi et al., 2005; Zhang et al., 2005; Braida et al., 2007; 2008; 2009; Baker et al., 2009). However, few studies have aimed at comparing the anti-addiction pharmacology of Sal A to traditional KOPr agonists.

Sal A has been shown to attenuate cocaine prime induced reinstatement and suppress cocaine induced hyperactivity in laboratory animals (Chartoff et al., 2008; Morani et al., 2009). We have shown in this study that the Sal A induced attenuation of cocaine reinstatement has no effect on either natural reward reinforcements or suppression of motor function (Morani et al., 2009). On the other hand, the classical KOPr agonists U50488H and spiradoline attenuated cocaine produced behaviours in a non-selective manner (Glick et al., 1995; Wadenberg, 2003). A study by Chavkin and 
colleagues (2004) showed that Sal A had a similar efficacy for KOPr to DYN whereas; it was more potent than both U50488H and U69593. Also the same study showed that Sal A had more affinity for KOPr compared to U50488H and U69593 (Chavkin et al., 2004). As Sal A has a different structure to traditional KOPr agonists (Fig 1.5), we hypothesized that Sal A might produce a better tolerated anti-addiction profile compared to classical KOPr agonists. If so, then compounds based on the structure of Sal A possessing better pharmacokinetics could be developed as potential anti-addiction agents. With this as a central hypothesis of this thesis, initial preliminary screenings were designed for Sal A, its structural analogues and nalmefene (MOPr antagonist/ partial KOPr agonist) aimed at exploring their behavioural anti-addiction pharmacology. The behavioural screening was extended further to measure the adverse effect profile of these KOPr compounds.

\subsection{Pharmacological profiling of novel KOPr agonists.}

The behavioural experiments used to screen Sal A, its analogues and nalmefene is shown in Fig 4.2. The initial aim of this thesis was to determine if Sal A, its structural analogues (DS1, MOM Sal B, EOM Sal B, herkinorin) and nalmefene produced antiaddiction behaviours in a similar way to traditional KOPr agonists. This was done using the cocaine prime-induced within reinstatement model (Fig 4.2). This model was initially reported by Worley et al, (1994) and has been used previously to screen traditional KOPr agonists for anti-addiction effects (Schenk et al., 1999; 2000; Morani et al., 2009). Our results show that Sal A $(0.3,1.0 \mathrm{mg} / \mathrm{kg}), \mathrm{DS} 1(0.3,1.0 \mathrm{mg} / \mathrm{kg}), \mathrm{MOM}$ Sal B $(0.3 \mathrm{mg} / \mathrm{kg})$ and nalmefene $(0.3,1.0 \mathrm{mg} / \mathrm{kg})$ all significantly attenuate cocaine prime induced drug seeking (Tab 4.1).

Once these compounds were shown to attenuate cocaine-seeking, the next step was to determine the selectivity in attenuation of cocaine-seeking behaviour. To do this 
we determined if sedative effects occurred. Motor function was determined by measuring cocaine produced hyperactivity in animals self-administering cocaine and the suppression of natural reward seeking behaviour was measured using the sucrose reinforcement test (Morani et al., 2009; Fig 4.2). Our results show that none of the compounds tested (Sal A, DS1, MOM Sal B, nalmefene) produced motor suppression during the reinstatement tests (Tab 4.1). Also, Sal A, DS1 and nalmefene pre-treatment did not suppress sucrose reinforcement. However MOM Sal B did decrease sucrose reinforcement (Tab 4.1). These results showed us that Sal A, DS1 and nalmefene induced attenuation of cocaine-seeking behaviour in a selective manner.

The compounds which selectively attenuated cocaine seeking behaviour were further tested for their pharmacological activation of KOPr in attenuating cocaine seeking Our results showed that Sal A, DS1 and nalmefene induced attenuation of cocaine-seeking were mediated via KOPr activation (Tab 4.1) and this effect was prevented when KOPr activation was blocked by selective KOPr antagonist nor-BNI (Fig 4.2).

These KOPr compounds were also tested for their behavioural adverse effects. Since KOPr agonists have been shown to cause aversion, motor suppression and depressive effects, we applied CTA, spontaneous open field activity and FST respectively to test these behaviours (Fig 4.2). Sal A and DS1 pre-treatment did not suppress motor function or produce taste aversions (Tab 4.1). Although, nalmefene did not produce taste aversions, it significantly suppressed motor function (Tab 4.1). However, all three compounds produced depressive like effects in FST (Tab 4.1). These data showed that Sal A and DS1 showed better adverse effect profile than nalmefene.

The effect of blocking SERT on Sal A and DS1 induced depressive behaviour in FST was also assessed (Fig 4.2). SERT antagonism via sub-chronic FLX treatment significantly improved Sal A and DS1 induced depressive effects in the FST (Tab 4.1). 
To further quantify the effect of Sal A, DS1 and U50488H on modulating SERT function, we used in vitro $\mathrm{ASP}^{+}$uptake paradigm in cells (Fig 4.2). Sal A and DS1 had no effect on SERT function, whereas U50488H pre-treatment decreased SERT function. This indicates a potential difference in the modulation of SERT function by classical and Sal A like KOPr agonists (Fig 4.2; Tab 4.1). However further studied are needed to clarify this possibility.

The effects of Sal A and DS1 were also tested for cocaine produced locomotion (acute, behavioural sensitization and stereotypy) to further characterize the behavioural effects of these compounds (Fig 4.2). Acute DS1 pre-treatment suppressed the expression of cocaine behavioural sensitization and locomotion activity following acute cocaine exposure (Tab 4.1). Although, Sal A attenuated cocaine sensitization, it potentiated motor function induced by acute cocaine (Tab 4.1). Overall, from this pharmacological profiling, we fleshed out three lead compounds Sal A, DS1 and nalmefene which can be further characterized for their anti-addiction effects. 


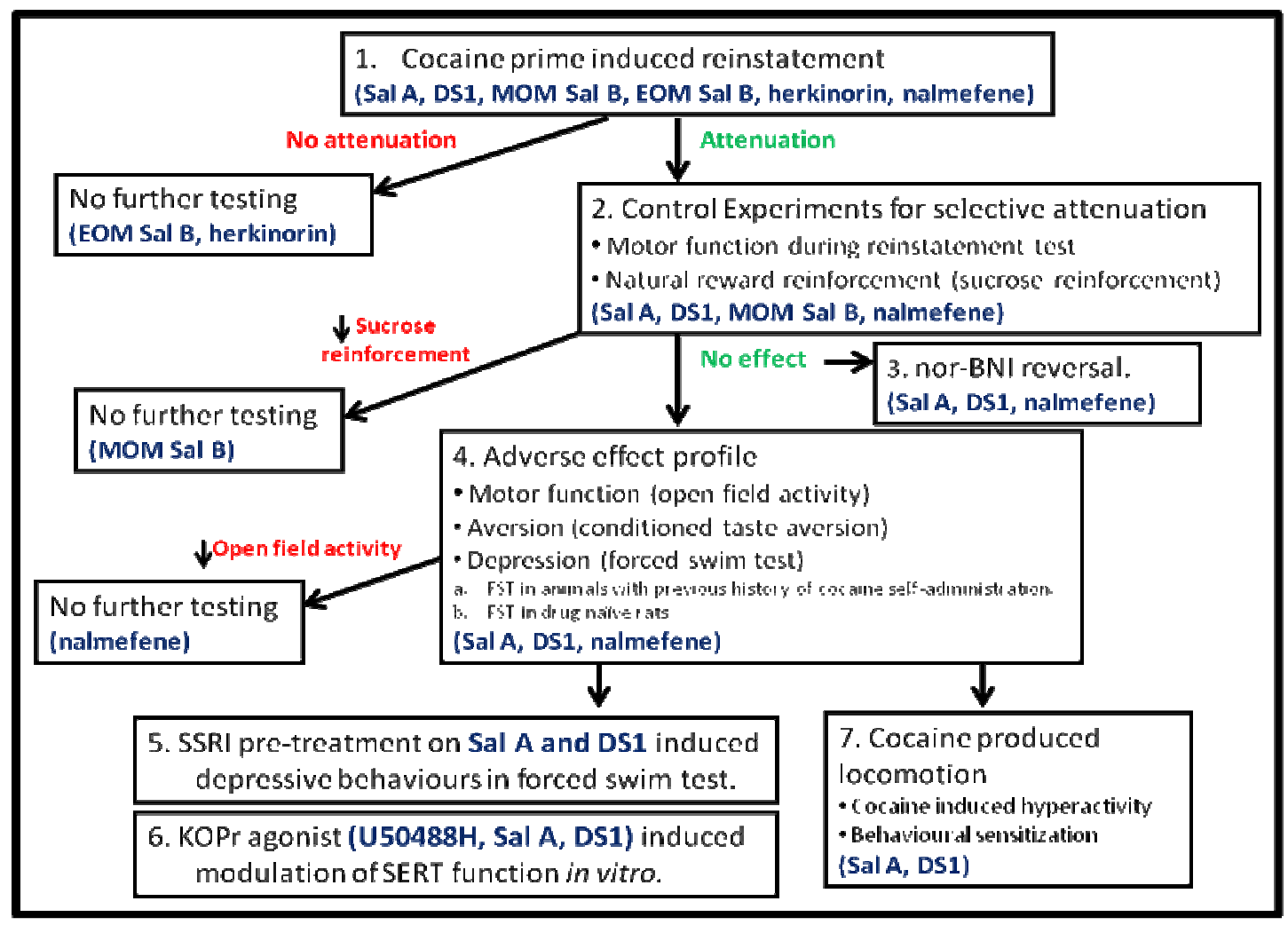

\section{Fig 4.2. Pharmacological profiling of novel KOPr agonists.}

Novel KOPr agonists were initially screened for their anti-cocaine profile followed by their selectivity in attenuation of cocaine-seeking. The compounds were further tested for their behavioural adverse effects. KOPr agonists Sal A and DS1 were also tested for their role in modulating serotonin transporter function and cocaine produced locomotion activity. 


\section{Tab 4.1. Pharmacological screening of KOPr agonists}

\begin{tabular}{|c|c|c|c|c|c|c|}
\hline & Sal A & DS1 & $\begin{array}{c}\text { MOM } \\
\text { Sal B }\end{array}$ & $\begin{array}{l}\text { EOM } \\
\text { Sal B } \\
\end{array}$ & Herkinorin & Nalmefene \\
\hline \multicolumn{7}{|l|}{$\begin{array}{c}\text { ANTI-ADDICTION } \\
\text { PROFILE }\end{array}$} \\
\hline $\begin{array}{c}\text { Cocaine induced } \\
\text { reinstatement }\end{array}$ & $\nabla$ & $\sqrt{1}$ & 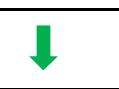 & NE & NE & $\downarrow$ \\
\hline $\begin{array}{l}\text { Motor suppression during } \\
\text { reinstatement test. }\end{array}$ & $\mathbf{N E}$ & NE & NE & - & - & NE \\
\hline Sucrose reinforcements & $\mathrm{NE}$ & $\mathrm{NE}$ & $\downarrow$ & - & - & $\mathrm{NE}$ \\
\hline $\begin{array}{c}\text { Cocaine induced } \\
\text { hyperactivity }\end{array}$ & 1 & 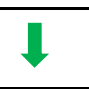 & & & & \\
\hline Behavioural Sensitization & 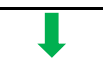 & 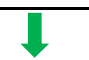 & & & & \\
\hline \multicolumn{7}{|l|}{$\begin{array}{c}\text { ADVERSE EFFECT } \\
\text { PROFILE }\end{array}$} \\
\hline Motor function & $\mathbf{N E}$ & NE & NE & - & - & $\uparrow$ \\
\hline Taste aversion & $\overline{N E}$ & $\overline{C T P}$ & & - & - & NE \\
\hline Immobility time in FST & $\mathbf{1}$ & $\boldsymbol{1}$ & - & - & - & $\widehat{\uparrow}$ \\
\hline $\begin{array}{c}\text { Effect of FLX pre- } \\
\text { treatment on immobility }\end{array}$ & $\boldsymbol{I}$ & 1 & & & & \\
\hline $\begin{array}{l}\text { Effect on ASP+ } \\
\text { accumulation }\end{array}$ & NE & NE & & & & \\
\hline
\end{tabular}

- attenuates/suppresses; - potentiates/ produces; NE - no effect

Effect of KOPr agonists were tested for their anti-addiction profile and side effect profile. KOPr agonists Sal A and DS1, having similar affinity for KOPr produced similar behavioural anti-addiction profile and adverse effect profile. More potent and long lasting Sal A analogue, MOM Sal B produced anti-addiction behaviour but also produced anhedonia. Mixed KOPr compound herkinorin had no effect on cocaine induced reinstatement. MOPr antagonist/ partial KOPr agonist, nalmefene produced anti-addiction behaviours without producing anhedonia and aversion. However, nalmefene produced motor suppression and depressive effects when tested in drug naive rats. 


\subsection{Significance of current results}

In Tab 4.2, we compare the binding affinity and efficacy at KOPr (and MOPr for nalmefene and herkinorin) with the KOPr compounds ability to attenuate cocaine seeking behaviours. Both Sal A and DS1 which have a similar affinity for- and potency at- KOPr successfully attenuated cocaine seeking behaviour in a selective manner (Tab 4.2). The dose at which both these compounds attenuated cocaine reinstatement was also similar $(0.3,1.0 \mathrm{mg} / \mathrm{kg})$. However, longer acting and more potent Sal A analogues such as EOM Sal B and MOM Sal B were ineffective in modulating cocaine seeking behaviour in a desirable manner. At $0.3 \mathrm{mg} / \mathrm{kg}$ dose, EOM Sal B showed a trend to increase responding which was an opposite effect to Sal A. Although, MOM Sal B attenuated cocaine-seeking at $0.3 \mathrm{mg} / \mathrm{kg}$ dose, this effect was non selective as it also suppressed natural reward. Therefore, the ability of neoclerodane diterpene KOPr agonists to selectively modulate cocaine induced behaviour may be dependent on the KOPr potency of these compounds.

It has been hypothesized that KOPr ligands with mixed affinity for other opioid receptors have the potential to be developed as anti-addiction pharmacotherapies (Stevenson et al., 2004; Archer et al., 1996; Bart et al., 2005; Kreek et al., 2005). With this in mind we performed an initial preliminary screening of nalmefene (MOPr antagonist/partial KOPr agonist) and herkinorin (MOPr/KOPr agonist) using cocaine prime induced drug seeking model. Acute nalmefene attenuated cocaine induced reinstatement in rats without suppressing sucrose reinforcements or producing taste aversion. Nalmefene has shown to bind with higher affinity to KOPr than MOPr and is an antagonist at MOPr (Bart et al., 2005; Tab 4.2). The endogeneous MOPr and KOPr activation produces opposing tonic effects (Pan, 1998). Additionally, as mentioned in Chapter 1 (Section 1.6), KOPr agonists and MOPr antagonists attenuated MOPr agonist-induced rewarding behaviours and -increase in DA levels in the NAc (Spanagel 
et al., 1992). Therefore, nalmefene may have an application in modulating the progression and development of cocaine addiction.

Considerable evidence supports the idea for the development of compounds that reverse the decrease in central monoamine activity as they are have potential abilities in preventing relapse following withdrawal from chronic drug use. Ideally by activating DA and 5HT receptors (Gorelick et al., 2004; Grabowski et al., 2004; Karila et al., 2008; Rothman et al., 2008). However, serious adverse effects associated with agonists at monoamine transporters have also opposed this theory (Mello and Negus, 2000). Developing agents which indirectly modulate monoaminergic systems may decrease the adverse effects associated with direct monoaminergic activators (Mello and Negus, 2000; Lin et al., 2010). We show in this study that acute herkinorin treatment has no effect on cocaine-seeking (Tab 4.1). Although a higher dose of herkinorin $(0.3,1.0$ $\mathrm{mg} / \mathrm{kg}$ ) showed a trend to suppress responses in a non-significant manner (Tab 4.2). As herkinorin binds with greater affinity to MOPr than KOPr and is an agonist at both the opioid receptors (Tidgewell et al., 2006; Tab 4.2), it has potential to be developed as a maintenance therapy that may prevent relapse. Thus, both nalmefene and herkinorin could potentially have an application as anti-addictives but at different stages of the addiction cycle. Further studies, are needed to test this hypothesis.

$\mathrm{KOPr}$ activation is associated with aversive and depressive effects (Carlezon et al., 1998; 2006; Todtenkopff et al., 2004; Mague et al., 2003; Ebnar et al., 2010) and the current results support this (Chapter 3; Tab 4.2). All three compounds tested showed depressive behaviours in drug naïve as well as cocaine exposed animals (Tab 3.3). The cocaine exposed animals were however abstinent from cocaine for a longer period of time (Tab 3.1). Abstinence from cocaine use has been shown to produce neuroadaptations which results in depressive behaviours (Carlezon et al., 1998; Nestler and Carlezon, 2006). Therefore, it might be possible that the depressive effects 
produced in cocaine self-administering animals might be an additive effect of cocaine abstinence and KOPr activation. As KOPr agonists are proposed to break the hedonic stage of addiction cycle (Shippenberg et al., 2007; Mysels and Sullivan, 2009). A detailed study is required to evaluate the depressive effects produced by acute KOPr exposure in self-administering rats which have not been subjected to any abstinence or withdrawal. In the present thesis, FLX pre-treatment attenuated the time spent immobile in animals exposed to Sal A and DS1. This finding has the potential to reduce the depressive effects produced by KOPr agonists when used to break the addiction cycle. Moreover, recent findings suggest that FLX $(3,10 \mathrm{mg} / \mathrm{kg})$ attenuates cue-induced and cocaine prime induced reinstatement in rats self-administering cocaine (Burmeister et al., 2003). Therefore, it would be interesting to determine the effect of co-administration of KOPr agonists with FLX on cocaine prime induced reinstatement and selfadministration. It would also be interesting to see if this proposed combination of treatments modulates central monoamine neurotransmission. This study may identify potential solution to the depressive side-effects associated with the use of KOPr agonists as anti-addiction pharmacotherapies.

Initial $\mathrm{ASP}^{+}$uptake experiments identify a potential difference in the modulation of SERT by classical- and neoclerodane diterpene-KOPr agonists (Tab 4.1). Therefore, detailed studies are required to explain the role of the 5HT system modulation by classical and novel KOPr agonists and associate them to the observed depressive behaviours. Such studies could utilise in vitro models to allow manipulation of signalling systems and proteins.

In conclusion, this study has identified Sal A, DS1 and nalmefene as lead compounds which can be further characterized for their anti-addiction profile. Furthermore, Sal A and DS1 attenuated cocaine behavioural sensitization. One of the aims of this thesis was to identify if any longer-acting structural analogues of Sal A 
could retain anti-addiction effects with fewer adverse effects. Our findings indicate that DS1 may be such an analogue. The favourable results with Sal A, DS1 and nalmefene from this thesis encourages a detailed behavioural profiling for their anti-addiction- and adverse-effects. Moreover, this study has utilised several experimental models to pharmacologically screen KOPr compounds for their anti-addiction behaviour. Using these preliminary experiments (Fig 4.2), we aim to screen more novel analogues for their anti-addiction pharmacology. This line of research has the potential to identify novel neoclerodane diterpenes as possible leads for the development of pharmacotherapies to treat psychostimulant addiction. 


\section{cocaine seeking behaviour.}

\begin{tabular}{|c|c|c|c|c|c|}
\hline Compound & $\begin{array}{l}\text { Binding } \\
\text { affinity at } \\
\text { KOPr Ki} \\
(\mathbf{n M})\end{array}$ & $\begin{array}{l}\text { Binding } \\
\text { affinity at } \\
\text { MOPr Ki } \\
(\mathrm{nM})\end{array}$ & $\begin{array}{l}\text { Potency } \\
\text { at KOPr } \\
\text { EC }_{50} \\
(\mathbf{n M})\end{array}$ & $\begin{array}{l}\text { Potency } \\
\text { at MOPr } \\
(\mathbf{n M})\end{array}$ & $\begin{array}{c}\text { Cocaine seeking } \\
\text { behaviour dose } \\
\text { (mgkg) }\end{array}$ \\
\hline Salvinorin A & $\begin{array}{l}2.4 \pm 0.4^{\mathrm{b}} \\
1.9 \pm 0.2^{\mathrm{a}}\end{array}$ & $>10000$ & $\begin{array}{l}1.8 \pm 0.5^{\mathrm{c}} \\
40 \pm 10^{\mathrm{d}}\end{array}$ & & $\begin{array}{c}0.3,1.0 \text { (selective } \\
\text { attenuation) }\end{array}$ \\
\hline DS-1 & $2.3 \pm 0.1^{\mathrm{a}}$ & $6820 \pm 660^{\mathrm{a}}$ & $30 \pm 5^{\mathrm{d}}$ & & $\begin{array}{c}0.3,1.0 \text { (selective } \\
\text { attenuation) }\end{array}$ \\
\hline Nalmefene & $\begin{array}{l}0.083 \pm \\
0.0008^{b}\end{array}$ & $0.24 \pm 0.006^{b}$ & $\begin{array}{c}\mathrm{EC}_{50}= \\
4.2 \pm 1.6^{\mathrm{c}} \\
\mathrm{IC}_{50}= \\
18 \pm 1.0^{\mathrm{c}}\end{array}$ & $\begin{array}{c}\mathrm{IC}_{50}= \\
13 \pm 2.3^{\mathrm{c}}\end{array}$ & $\begin{array}{c}0.3,1.0 \text { (selective } \\
\text { attenuation) }\end{array}$ \\
\hline EOM Sal B & $0.32 \pm 0.02^{b}$ & ND & $0.14 \pm 0.01^{\mathrm{c}}$ & & $\begin{array}{l}0.03 \text { (no effect); } 0.1 \text {, } \\
0.03 \text { (trend to } \\
\text { increase responding) }\end{array}$ \\
\hline MOM Sal B & $0.6 \pm 0.07^{b}$ & ND & $0.40 \pm 0.04^{\mathrm{c}}$ & & $\begin{array}{c}0.03,0.1 \text { (trend to } \\
\text { increase responding) } \\
0.3 \text { (non-selective } \\
\text { attenuation) }\end{array}$ \\
\hline Herkinorin & $90 \pm 2^{a}$ & $12.1 \pm 1^{\mathrm{a}}$ & $1320 \pm 150^{\mathrm{d}}$ & & $\begin{array}{c}0.3,1.0 \text { (non- } \\
\text { significant trend to } \\
\text { decrease responding) }\end{array}$ \\
\hline
\end{tabular}

${ }^{a}$ Data expressed as $\pm S D ;{ }^{b}$ Data expressed as $\pm S E M ; N D=$ not defined. ${ }^{c}$ Enhancement of $\left[{ }^{35} S\right] G T P \gamma S$ binding to Chinesse Hamster Ovary - human KOPr; EC50 $\pm S E M$ (nM). $\left.{ }^{d}{ }^{35} S\right] G T P \gamma S$ functional assay carried out in stably transfected Chinesse Hamster Ovary cells containing DNA for human KOPr;EC50 \pm SD nM. Data for Sal A, DS1 and herkinorin, modified from Harding et al., 2005; data for MOM Sal B, EOM Sal B, modified from Munro et al., 2008; data for nalmefene modified from Bart et al., 2005. 


\subsection{Future directions}

Results obtained from this thesis have opened new avenues to further quantify the behavioural anti-addiction and side effect profiles for the lead compounds Sal A, DS1 and nalmefene. Additionally, there is a need to determine the mechanisms by which these compounds produced their anti-addiction and depressive behaviours. The following section gives a brief description of future possible studies with these compounds.

\section{Effect of KOPr agonists in models of cocaine self-administration and reinstatement.}

As discussed in Chapter 2 (Section 2.5, discussion), there is a need to test the lead KOPr compounds using models which show a well defined set of alterations in neuronal adaptations seen in pre-clinical studies (Self et al., 2004). To do this additional studies will be required to test the effects of Sal A, DS1 and nalmefene on cocaine prime induced reinstatement following extended extinction times or utilising a betweensessions reinstatement paradigm (Stretch et al., 1971; Ferragud et al., 2009). Keeping in mind the rationale for using KOPr agonists as cessation agents for cocaine selfadministration, it would be imperative to test the effects of these lead compounds (Sal A, DS1 and nalmefene) on cocaine self-administration in animals' stably selfadministering cocaine (Glick et al., 1996). Also, it would be interesting to observe the effects of these lead compounds on altering the monoamine transporter functions in animals self-administering cocaine. This can be achieved by measuring DAT and SERT function by using rotating disc electrode voltammetry (RDEV) in NAc and CPU brain regions. These tests would idealy be performed on animals following cocaine selfadministration and reinstatement tests. 


\section{Endogenous KOPr antagonism on cocaine prime induced reinstatement.}

As mentioned in Chapter 2 (Section 2.5, discussion), KOPr antagonism by norBNI reversed KOPr agonists induced attenuation of drug seeking behaviour. However, the effect of KOPr antagonism by itself on cocaine-seeking was not performed. Ideally this can be measured by determining the effects of KOPr antagonism with traditional (nor-BNI, JDTic) and novel antagonists on cocaine-prime induced -within-session reinstatement and -reinstatement after prolonged period of abstinence in rats (Worley et al., 1994; Beardsley et al., 2005). Such experiments could prove useful, as KOPr antagonists are proposed as maintainence agents to prevent relapse (Section 4.1). Also, determining the DAT and SERT functions in NAc and CPU regions by using RDEV in animals subjected to the reinstatement tests could help explain the mechanism of the observed behaviours.

\section{Effect of KOPr antagonists on stressors induced cocaine seeking.}

As explained in Section 4.1, reinstatement of extinguished cocaine selfadministration by presentation of environmental stressors has been used for pharmacological evaluation of KOPr antagonists. Therefore, this paradigm could be useful in pharmacological screening of novel KOPr antagonists as agents to prevent relapse induced by stress. The reinstatement of cocaine-seeking could be initiated by the presentation of foot-shock stressors or forced swim stress in rats (Beardsley et al., 2005; McLaughlan et al., 2005a; 2005b). Also, determining the function of DAT and SERT in the NAc and CPU could help explain the mechanism of KOPr antagonists in this model. Such experiments could be performed using RDEV following reinstatement testing. 


\section{Effect of KOPr agonists on FST in cocaine self-administering animals.}

As mentioned earlier (Section 4.4), KOPr agonists have shown to produce depressive like symptoms (increased immobility time) in animals self-administering cocaine (Fig 3.6; Tab 3.3; Chapter 3). However, these rats were abstained from cocaine (Tab 3.1). Therefore, in order to quantify the depressive effects produced by KOPr activation, effects of Sal A, DS1 and nalmefene need to be measured using FST in cocaine self-administering animals which have not been subjected to any abstinence. In addition to the measurement of the behaviours, determining the DAT, SERT function in brain regions subjected to reward (dorsal and ventral striatum) could explain the mechanism of depressive effects following KOPr activation (Section 1.5.5.3; 1.5.5.4). These experiments could be performed using the RDEV technique.

\section{Effect of KOPr agonists on cocaine motor sensitization after prolonged abstinence.}

The results from this present study showed the efficacy of both Sal A and DS1 to attenuate expression of locomotion sensitization when animals were challenged with the cocaine injection after a short period of abstinence (4 days). Previous reports suggest that expression of sensitization is a model which represents alteration in underlying neuronal circuits mediating psychomotor function and craving potential of the psychostimulants (Stekette, 2005; Robinson and Berridge, 2000; 2003; 2008). Also, challenging sensitized animals with a low dose of cocaine even after a long period of abstinence has shown to express locomotor sensitization (Heidbreder et al., 1995). Therefore, it would be interesting to test the effect of Sal A and DS1 on expression of sensitization after a prolonged period of abstinence (15, 30, 45 days). Additionally, the DAT and SERT function in NAc and CPU could be determined in these sensitized animals using RDEV. This experiment has the potential to provide evidence to link the 
alteration induced by KOPr agonists in the monoamine transporter function at midbrain reward circuit with cocaine motor sensitization.

\section{Effect of KOPr agonists on cocaine induced stereotypy.}

KOPr agonists, Sal A and DS1 have shown to attenuate motor sensitization produced by high dose of cocaine (20 mg/kg; Tab 4.2). However, both Sal A and DS1 pre-treatment showed a non-significant enhancement in cocaine-induced stereotypy (Fig 2.4c, 2.8c). As suppression in forward locomotion by KOPr agonists might reflect an enhanced stereotypy, a detailed study on cocaine produced stereotypic behaviours is warranted. With similar experimental procedure to induce motor sensitization (mentioned in Section 2.2; Chapter 2), effect of KOPr compounds on cocaine produced stereotypic behaviours could be measured. The stereotypic behavioural counts could be determined using methods described by Kalivas et al., (1988) and Szumlinski et al., (2000).

\section{Effect of SSRI+ KOPr agonists in cocaine prime induced drug-seeking}

Both KOPr agonist and SERT antagonist, FLX treatment has shown to attenuate cocaine prime induced reinstatement in laboratory animals (Burmeister et al., 2003). Moreover, sub-chronic FLX pre-treatment attenuated KOPr agonists produced depressive behaviours in FST (Tab 4.1). Therefore, it would be interesting to test the effect of co-administration of FLX with Sal A/DS1 on cocaine-seeking behaviour. To test this, an initial screening could be performed using the within-session reinstatement model (Worley et al., 1994). Preliminary results from such experiment could open a new avenue of research using SERT antagonists in combination with KOPr agonists in tackling cocaine addiction without producing KOPr activation induced depressive effects. 


\section{Pre-clinical models to quantify behavioural despair induced by KOPr agonists}

With the limitations of the FST paradigm (discussed in Chapter 3; Section 3.5), it would be interesting to characterize KOPr activation produced behavioural despair using paradigms which have been previously applied to study anhedonia pre-clinically. Such tests could be performed using social interaction effect (Sams-Dodd, 1997), sucrose drinking (Muscat and Wilner, 1992) and ICSS test in rats (Todtenkopff et al., 2004; Ebnar et al., 2010). The measurement of ICSS thresholds have been previously used to evaluate anhedonia like effects of Sal A (Ebnar et al., 2010) and hence could be useful in evaluating DS1 and nalmefene produced behavioural despair.

\section{Herkinorin as maintenance therapy}

Herkinorin is the only synthetic Sal A analogue with more affinity for MOPr and weak affinity for KOPr. Also, herkinorin is an agonist at MOPr and KOPr. The results from this thesis showed that herkinorin did not potentiate cocaine seeking in within session reinstatement test ( $\mathrm{Tab} 4.1)$. This led to the hypothesis that neoclerodane diterpenes acting as agonists at $\mathrm{MOPr}$ and $\mathrm{KOPr}$ could be effective in relapse prevention. To prove this hypothesis, experiments could be performed by looking at the effect of herkinorin pre-treatment on cocaine prime induced reinstatement after a prolonged period of withdrawal (Ferragud et al., 2009). Also, by measuring the function of DAT and SERT in NAc and CPU could help explain the mechanism of action of herkinorin. Such experiments could be performed using RDEV technique. The preliminary results from such experiments with herkinorin have the potential to develop a new class of neoclerodanes diterpenes aimed at preventing relapse to cocaine use. 


\subsection{Summary and conclusions}

This study shows that the novel KOPr activating neoclerodane diterpenes Sal A, DS1 and MOM Sal B attenuate cocaine induced behaviours in a similar way to traditional KOPr agonists. This effect was also observed for partial KOPr agonist/MOPr antagonist, nalmefene. However, longer acting and more potent Sal A analogue, MOM Sal B produced anhedonia which was not seen with either Sal A, DS1 or nalmefene. Also, Sal A and DS1 showed better adverse effect profile than nalmefene in drug naive rats. However, all three compounds tested (Sal A; DS1 and nalmefene) produced depression and preliminary evidence has been presented to suggest that Sal A and DS1 induced depression may involve serotonin neurotransmission. Finally, preliminary in vitro cell studies show KOPr activation by traditional agonist U50488H directly modulates serotonin transporter function. However, Sal A analogues may regulate SERT in a different manner compared to traditional KOPr agonists. Further studies are needed to determine the extent of these differences. 


\section{References}

- Addiction. (2011). In Merriam-Webster.com. Retrived May 27, 2011, from http://www.merriam-webster.com/medlineplus/addiction.

- Andersen SL, Gazzara RA. (1993). The ontogeny of apomorphine-induced alterations of neostriatal dopamine release: effects on spontaneous release. $J$ Neurochem, 61:2247-55.

- Ansonoff MA, Zhang J, Czyzyk T, Rothman RB, Stewart J, Xu H, et al. (2006). Antinociceptive and Hypothermic Effects of Salvinorin A Are Abolished in a Novel Strain of kappa-Opioid Receptor-1 Knockout Mice. J Pharmacol Exp Ther, 318: 641-648.

- Archer S., Glick S, Bidlack J. (1996). Cyclazocine revisited. Neurochem Res, 21:1369-1373.

- Auriacombe M, Reneric JP, Le Moal M. (1997). Animal models of anhedonia. Psychopharmacology, 134: 337-338.

- Baker J, Jatlow P, Pade P, Ramakrishnan V, McCance-Katz EF. (2007). Acute cocaine responses following cocaethylene infusion. Am J Drug Alcohol Abuse, $33: 619-25$.

- Baker L, Panos J, Killinger B, Peet M, Bell L, Haliw L, et al. (2009). Comparison of the discriminative stimulus effects of salvinorin $\mathrm{A}$ and its derivatives to U69593 and U50488 in rats. Psychopharmacology 203:203-211.

- Bals-Kubik R, Herz A, Shippenberg TS. (1989). Evidence that the aversive effects of opioid antagonists and $\kappa$-agonists are centrally mediated. Psychopharmacology, 98: 203-206.

- Bals-Kubik R, Ableitner A, Herz A, Shippenberg TS. (1993). Neuroanatomical sites mediating the motivational effects of opioids as mapped by the conditioned place preference paradigm in rats. J Pharmacol Exp Ther, 264, 489-495. 
- Barsan WG, Seger D, Danzl DF, Ling LJ, Bartlett R, Buncher R, et al. (1989). Duration of antagonistic effects of nalmefene and naloxone in opiate-induced sedation for emergency department procedures. Am J Emerg Med, 7:155-161.

- Bart G, Schluger JH, Borg L, Ho A, Bidlack JM, Kreek MJ. (2005). Nalmefene Induced Elevation in Serum Prolactin in Normal Human Volunteers: Partial Kappa Opioid Agonist Activity? Neuropsychopharmacology, 30: 2254-2262.

- Battaglia, G., Sharkey, J, Kuhar, MJ and Errol B. de Souza. (1991). Neuroanatomic specificity and time course of alterations in rat brain serotonergic pathways induced by MDMA (3,4methylenedioxymethamphetamine): Assessment using quantitative autoradiography. Synapse, 8: 249-260.

- Baumann M, Rothman R. (1998). Alterations in serotonergic responsiveness during cocaine withdrawal in rats: similarities to major depression in humans. Biol Psychiatry, 44: 578-591.

- Baumann MH, Rothman RB, Pablo JP, Mash DC. (2001). In vivo neurobiological effects of ibogaine and its O-desmethyl metabolite, 12hydroxyibogamine (noribogaine), in rats. J Pharmacol Exp Ther, 297:531-9.

- Beardsley P, Howard J, Shelton K, Carroll F. (2005). Differential effects of the novel kappa opioid receptor antagonist, JDTic, on reinstatement of cocaineseeking induced by footshock stressors vs cocaine primes and its antidepressantlike effects in rats. Psychopharmacology, 183: 118-126.

- Beardsley P, Pollard G, Howard J, Carroll F. (2010). Effectiveness of analogs of the kappa opioid receptor antagonist (3R)-7-hydroxy-N-((1S)-1-\{[(3R,4R)-4-(3hydroxyphenyl)-3,4-dimethyl-1-piperidinyl]methyl -2-methylpropyl)-1,2,3,4tetrahydro-3-isoquinolinecarboxamide (JDTic) to reduce U50,488-induced 
diuresis and stress-induced cocaine reinstatement in rats. Psychopharmacology, 210: 189-198.

- Beuming T, Kniazeff J, Bergmann ML, Shi L, Gracia L, Raniszewska K, Newman AH, Javitch JA, Weinstein H, Gether U, Loland CJ. (2008). The binding sites for cocaine and dopamine in the dopamine transporter overlap. Nat Neurosci, 11:780-9.

- Béguin C, Richards MR, Wang Y, Chen Y, Liu-Chen LY, Ma Z, Lee DY, Carlezon WA Jr, Cohen BM. (2005). Synthesis and in vitro pharmacological evaluation of salvinorin A analogues modified at C(2). Bioorg Med Chem Lett, $15: 2761-5$.

- Béguin C, Potter DN, Dinieri JA, Munro TA, Richards MR, Paine TA, Berry L, Zhao Z, Roth BL, Xu W, Liu-Chen LY, Carlezon WA Jr, Cohen BM. (2008). Nmethylacetamide analog of salvinorin A: a highly potent and selective kappaopioid receptor agonist with oral efficacy. J Pharmacol Exp Ther, 324:188-95.

- Bellivier F, Laplanche JL, Leboyer M, Feingold J, Bottos C, Allilaire JF, Launay JM. (1997). Serotonin transporter gene and manic depressive illness: an association study. Biol Psychiatry, 41:750-2.

- Belcheva MM, Clark AL, Haas PD, Serna JS, Hahn JW, Kiss A, Coscia CJ. Mu and kappa opioid receptors activate ERK/MAPK via different protein kinase $\mathrm{C}$ isoforms and secondary messengers in astrocytes. J Biol Chem., 280:27662-9.

- Beninger R, Herz R. (1986). Pimozide blocks establishment but not expression of cocaine-produced environment-specific conditioning. Life Sci 38, 1425-1431.

- Bhargava HN, Gulati A, Ramarao P (1989) Effect of chronic administration of $\mathrm{U} 50488 \mathrm{H}$ on tolerance to its pharmacological actions and on multiple opioid receptors in rat brain regions and spinal cord. J Pharmacol Exp Ther 251:21-26 
- Bikbulatov RV, Yan F, Roth BL, Zjawiony JK. (2007). Convenient synthesis and in vitro pharmacological activity of 2-thioanalogs of salvinorins $\mathrm{A}$ and $\mathrm{B}$. Bioorg Med Chem Lett, 17: 2229-2232.

- Bhattacharyya AK, Pradhan SN.(1979). Interactions between motor activity and sterotypy in cocaine-treated rats. Psychopharmacology, 63:311-2.

- Blakely RD, De Felice LJ, Hartzell HC. (1994). Molecular physiology of norepinephrine and serotonin transporters. $J$ Exp Biol, 196: 263-281

- Blakely RD, DeFelice LJ. (2007). All Aglow about Presynaptic Receptor Regulation of Neurotransmitter Transporters. Mol Pharmacol, 71:1206-1208.

- Bolan EA, Kivell B, Jaligam V, Oz M, Jayanthi LD, Han Y, et al. (2007). D2 Receptors Regulate Dopamine Transporter Function via an Extracellular SignalRegulated Kinases 1 and 2-Dependent and Phosphoinositide 3 KinaseIndependent Mechanism. Mol Pharmacol, 71:1222-1232.

- Borgne A, Aubin HJ, Berlin I. (2004). Current therapeutic strategies in smoking cessation. Rev Prat, 54:1883-93.

- Borsini F, Meli A. (1988). Is the forced swimming test a suitable model for revealing antidepressant activity? Psychopharmacology, 94:147-160.

- Bowen CA, Negus SS, Zong R, Neumeyer JL, Bidlack JM, Mello NK. (2003). Effects of Mixed-Action kappa-mu Opioids on Cocaine Self-Administration and Cocaine Discrimination by Rhesus Monkeys. Neuropsychopharmacology, 28:1125-1139.

- Bozarth M. (1987). Ventral tegmental reward system. In L. Oreland and J. Engel (eds.) Brain Reward Systems and Abuse (pp. 1-17). New York: Raven Press.

- Bozarth MA, Wise RA. (1981). Intracranial self-administration of morphine into the ventral tegmental area in rats. Life Sci, 28:551-555. 
- Braida D, Limonta V, Pegorini S, Zani A, Guerini-Rocco C, Gori E, et al. (2007). Hallucinatory and rewarding effect of salvinorin A in zebrafish: $\kappa$-opioid and CB-1 cannabinoid receptor involvement. Psychopharmacology, 190, 441-448.

- Braida D, Limonta V, Capurro V, Fadda P, Rubino T, Mascia P, et al. (2008). Involvement of [kappa]-Opioid and Endocannabinoid System on Salvinorin AInduced Reward. Biol Psychiatry, 63, 286-292.

- Braida D, Capurro V, Zani A, Rubino T, Viganò D, Parolaro D, et al. (2009). Potential anxiolytic- and antidepressant-like effects of salvinorin A, the main active ingredient of Salvia divinorum in rodents. Br J Pharmacol 157:844-853.

- Broadbent J, Gaspard TM, Dworkin SI. (2002). Assessment of the discriminative stimulus effects of cocaine in the rat: Lack of interaction with opioids. Pharmacol Biochem Behav, 51:379-385.

- Bruchas MR, Macey TA, Lowe JD, Chavkin C. (2006). Kappa opioid receptor activation of p38 MAPK is GRK3- and arrestin-dependent in neurons and astrocytes. J Biol Chem, 281:18081-9.

- Bruchas MR, Land BB, Aita M, Xu M, Barot SK, Li S, et al. (2007). StressInduced p38 Mitogen-Activated Protein Kinase Activation Mediates kappaOpioid-Dependent Dysphoria. J. Neurosci., 27:11614-11623.

- Bruchas MR, Land BB, Chavkin C. (2010). The dynorphin/kappa opioid system as a modulator of stress-induced and pro-addictive behaviors. Brain Res, $1314: 44-55$.

- Bruijnzeel AW. (2009). kappa-Opioid receptor signaling and brain reward function. Brain Res Rev, 62:127-146.

- Burmeister JJ, Lungren EM, Neisewander JL. (2003). Effects of fluoxetine and d-fenfluramine on cocaine-seeking behavior in rats. Psychopharmacology, 168:146-54. 
- Butelman E, Harris T, Kreek M. (2004). The plant-derived hallucinogen, salvinorin A, produces kappa-opioid agonist-like discriminative effects in rhesus monkeys. Psychopharmacology, 172: 220-224.

- Butelman ER, Mandau M, Tidgewell K, Prisinzano TE, Yuferov V, Kreek MJ. (2007). Effects of Salvinorin A, a kappa-Opioid Hallucinogen, on a Neuroendocrine Biomarker Assay in Nonhuman Primates with High kappaReceptor Homology to Humans. J Pharmacol Exp Ther, 320:300-306.

- Butelman ER, Rus S, Simpson DS, Wolf A, Prisinzano TE, Kreek MJ. (2008). The Effects of Herkinorin, the First Mu-Selective Ligand from a Salvinorin ADerived Scaffold, in a Neuroendocrine Biomarker Assay in Nonhuman Primates. J Pharmacol Exp Ther, 327:154-160.

- Butelman ER, Prisinzano TE, Deng H, Rus S, Kreek MJ (2009). Unconditioned behavioral effects of the powerful kappa-opioid hallucinogen salvinorin $\mathrm{A}$ in nonhuman primates: fast onset and entry into cerebrospinal fluid. $J$ Pharmacol Exp Ther 328:588-597.

- Butelman E, Rus S, Prisinzano T, Kreek M. (2010). The discriminative effects of the $\kappa$-opioid hallucinogen salvinorin $\mathrm{A}$ in nonhuman primates: dissociation from classic hallucinogen effects. Psychopharmacology, 210:253-262.

- Bylund DB, Toews ML. (1993). Radioligand binding methods: practical guide and tips. Am J Physiol Lung Cell Mol Physiol, 265:L421-L429.

- Caine SB, Koob GF. (1993). Modulation of cocaine self-administration in the rat through D-3 dopamine receptors. Science, 260:1814-6.

- Cappendijk SLT, Dzoljic MR. (1993). Inhibitory effects of ibogaine on cocaine self-administration in rats. Eur J Pharmacol, 241:261-265.

- Carlezon WA, Thome J, Olson VG, Lane-Ladd SB, Brodkin ES, Hiroi N, et al. (1998). Regulation of Cocaine Reward by CREB. Science, 282: 2272-2275. 
- Carlezon WA, Duman RS, Nestler EJ. (2005). The many faces of CREB. Trends Neurosci, 28:436-445.

- Carlezon WA Jr, Béguin C, DiNieri JA, Baumann MH, Richards MR, Todtenkopf MS, et al. (2006). Depressive-like effects of the kappa-opioid receptor agonist salvinorin A on behavior and neurochemistry in rats. $J$ Pharmacol Exp Ther 316:440-447.

- Carlezon WA, Thomas MJ. (2009). Biological substrates of reward and aversion: A nucleus accumbens activity hypothesis. Neuropharmacology, 56:122-132.

- Carlsson A. (1969). Pharmacology of Synaptic Monoamine Transmission. In K. Akert \& P. G. Waser (Eds.), Progress in Brain Research (Volume 31, pp. 53-59): Elsevier.

- Carr GV, Mague SD. (2008). p38: The Link between the kappa-Opioid Receptor and Dysphoria. J. Neurosci., 28:2299-2300.

- Carroll KM, Onken LS. Behavioral therapies for drug abuse. Am J Psychiatry. 2005;162:1452-1460.

- Carroll KM, Rounsaville BJ, Nich C, Gordon LT, Wirtz PW, Gawin FH. (1994). One year follow-up of psychotherapy and pharmacotherapy for cocaine dependence: Delayed emergence of psychotherapy effects. Arch Gen Psychiatry. 1994;51:989-997.

- Castagné V, Moser P, Porsolt RD.(2009). Behavioral Assessment of Antidepressant Activity in Rodents. Methods of Behavior Analysis in Neuroscience. 2nd edition. Buccafusco JJ, editor Boca Raton (FL): CRC Press. Chapter 6.

- Chang 1, Chronicle EP. (2007). Functional Imaging Studies in Cannabis Users. Neuroscientist, 13:422-432. 
- Chartoff EH, Potter D, Damez-Werno D, Cohen BM, Carlezon WA Jr (2008). Exposure to the selective kappa-opioid receptor agonist salvinorin A modulates the behavioral and molecular effects of cocaine in rats. Neuropsychopharmacol $33: 2676-2687$.

- Chartoff EH, Papadopoulou M, MacDonald ML, Parsegian A, Potter D, Konradi C, et al. (2009). Desipramine Reduces Stress-Activated Dynorphin Expression and CREB Phosphorylation in NAc Tissue. Mol Pharmacol, 75, 704-712.

- Chavkin C, James IF, Goldstein A. (1982). Dynorphin is a specific endogenous ligand of the kappa-opioid receptor. Science, 215:413-415.

- Chavkin C, Sud S, Jin W, Stewart J, Zjawiony JK, Siebert DJ, et al. (2004). Salvinorin A, an Active Component of the Hallucinogenic Sage Salvia divinorum Is a Highly Efficacious Kappa-Opioid Receptor Agonist: Structural and Functional Considerations. J Pharmacol Exp Ther, 308:1197-1203.

- Chefer V, Thompson AC, Shippenberg TS (1999). Modulation of cocaineinduced sensitization by kappa-opioid receptor agonists. Role of the nucleus accumbens and medial prefrontal cortex. Ann N Y Acad Sci 877:803-6.

- Chefer VI, Czyzyk T, Bolan EA, Moron J, Pintar JE, Shippenberg TS (2005). Endogenous kappa-opioid receptor systems regulate mesoaccumbal dopamine dynamics and vulnerability to cocaine. J Neurosci 25:5029-5037.

- Collins S, Gerdes RM, D'Addario C, Izenwasser S (2001). Kappa opioid agonists alter dopamine markers and cocaine-stimulated locomotor activity. Behav Pharmacol 12:237-245.

- Collins SL, D'Addario C, Izenwasser S. (2001). Effects of [kappa]-opioid receptor agonists on long-term cocaine use and dopamine neurotransmission. Eur J Pharmacol, 426:25-34. 
- Collins SL, Kunko PM, Ladenheim B, Cadet JL, Carroll FI, Izenwasser S. (2002). Chronic cocaine increases kappa-opioid receptor density: Lack of effect by selective dopamine uptake inhibitors. Synapse, 45:153-158.

- Connor M, Kitchen I. (2006). Has the sun set on kappa3-opioid receptors? Br $J$ Pharmacol, 147:349-350.

- Connor M, Christie MD. Opioid receptor signalling mechanisms. Clin Exp Pharmacol Physiol 1999;26:

- $\quad 493-499$.

- Corbett AD, Paterson SJ, McKnight AT, Magnan J, Kosterlitz HW. (1982). Dynorphin and dynorphin are ligands for the kappa-subtype of opiate receptor. Nature, 299:79-81.

- Cosgrove KP, Carroll ME. (2002). Effects of Bremazocine on SelfAdministration of Smoked Cocaine Base and Orally Delivered Ethanol, Phencyclidine, Saccharin, and Food in Rhesus Monkeys: A Behavioral Economic Analysis. J Pharmacol Exp Ther, 301:993-1002.

- Davis CM, Rice KC, Riley AL. (2009). Opiate-agonist induced taste aversion learning in the Fischer 344 and Lewis inbred rat strains: evidence for differential mu opioid receptor activation. Pharmacol Biochem Behav,93:397-405.

- Davis CM, Riley AL. (2010). Conditioned taste aversion learning: implications for animal models of drug abuse. Ann N Y Acad Sci, 1187:247-75.

- D'Addario C, Di Benedetto M, Izenwasser S, Candeletti S, Romualdi, P. (2007). Role of serotonin in the regulation of the dynorphinergic system by a kappaopioid agonist and cocaine treatment in rat CNS. Neuroscience, 144:157-164.

- D'Addario C, Di Benedetto M, Candeletti S, Romualdi P. The kappa-opioid receptor agonist U-69593 prevents cocaine-induced phosphorylation of DARPP- 
32 at $\operatorname{Thr}(34)$ in the rat brain. Brain Res Bull. 2007 Jun 15;73(1-3):34-9. Epub 2007 Feb 20.

- Daunais JB, Roberts DCS, McGinty JF. (1995). Short-term cocaine self administration alters striatal gene expression. Brain Res Bull, 37:523-527.

- De Vries TJ, Schoffelmeer AN, Binnekade R, Vanderschuren LJ. (1999). Dopaminergic mechanisms mediating the incentive to seek cocaine and heroin following long-term withdrawal of IV drug self-administration. Psychopharmacology, 143:254-60.

- de Wit H, Stewart J. (1981). Reinstatement of cocaine-reinforced responding in the rat. Psychopharmacology, 75:134-143.

- de Wit H, Stewart J. (1983). Drug reinstatement of heroin-reinforced responding in the rat. Psychopharmacology, 79:29-31.

- Deneau G, Yanagita T, Seevers MH. (1969). Self-administration of psychoactive substances by the monkey. Psychopharmacology, 16: 30-48.

- DeRubeis RJ, Crits-Christoph P. (1998). Empirically supported individual and group psychological treatments for adult mental disorders. J Consult Clin Psychol, 66:37-52.

- Detke M, Rickels M, Lucki I (1995). Active behaviors in the rat forced swimming test differentially produced by serotonergic and noradrenergic antidepressants. Psychopharmacology 121:66-72.

- Devine D, Leone P, Pocock D, Wise RA. (1993). Differential involvement of ventral tegmental mu, delta and kappa opioid receptors in modulation of basal mesolimbic dopamine release: in vivo microdialysis studies. J Pharmacol Exp Ther, 266, 1236-1246.

- Devine D, Wise R. (1994). Self-administration of morphine, DAMGO, and DPDPE into the ventral tegmental area of rats. J. Neurosci, 14:1978-1984. 
- Di Chiara G (1995). The role of dopamine in drug abuse viewed from the perspective of its role in motivation. Drug Alcohol Depend 38:95-137.

- Di Chiara G, Imperato A. (1988). Opposite effects of mu and kappa opiate agonists on dopamine release in the nucleus accumbens and in the dorsal caudate of freely moving rats. J Pharmacol Exp Ther, 244:1067-1080.

- Di Chiara G, Imperato A. (1988). Drugs abused by humans preferentially increase synaptic dopamine concentrations in the mesolimbic system of freely moving rats. Proc Natl Acad Sci USA, 85:5274-5278.

- DiNieri JA, Nemeth CL, Parsegian A, Carle T, Gurevich VV, Gurevich E, et al. (2009). Altered Sensitivity to Rewarding and Aversive Drugs in Mice with Inducible Disruption of cAMP Response Element-Binding Protein Function within the Nucleus Accumbens. J. Neurosci., 29:1855-1859.

- Dinsmoor J. (2004). The etymology of basic concepts in the experimental analysis of behavior. J Exp Anal Behav. 82:311-316.

- Downs, D. A., \& Woods, J. H. (1974). Codeine and cocaine reinforced responding in rhesus monkeys: Effect of dose on response rates under fixed-ratio schedule. J Pharmacol Exp Ther, 191:179-188.

- Drug Use in New Zealand: Key Results of the 2007/08. New Zealand Alcohol and Drug Use Survey. (2010). Ministry of Health, New Zealand.

- Ernst AM. (1966). Mode of action of apomorphine and dexamphetamine on gnawing compulsion in rats. Psychopharmacology, 10:316-23.

- Ebner S, Roitman M, Potter D, Rachlin A, Chartoff E (2010). Depressive-like effects of the kappa opioid receptor agonist salvinorin A are associated with decreased phasic dopamine release in the nucleus accumbens. Psychopharmacology 210:241-252. 
- Eddy N, Halbach H, Isbell H, Seevers MH. (1965). Drug Dependence: its Significance and Characteristics. Bull. Wld Hlth Org, 32:721-733.

- Eguchi M. (2004). Recent advances in selective opioid receptor agonists and antagonists. Med Res Rev, 24:182-212.

- Ehrenreich H, Krampe H. (2004). Does disulfiram have a role in alcoholism treatment today? Not to forget about disulfiram's psychological effects. Addiction, 99:26-7.

- Elkashef A, Fudala PJ, Gorgon L, Li SH, Kahn R, Chiang N, Vocci F, Collins J, Jones K, Boardman K, Sather M. (2006). Double-blind, placebo-controlled trial of selegiline transdermal system (STS) for the treatment of cocaine dependence. Drug Alcohol Depend, 85:191-7.

- Epstein DH, Preston KL, Stewart J, Shaham Y. (2006). Toward a model of drug relapse: An assessment of the validity of the reinstatement procedure. Psychopharmacology, 189:1-16.

- Esposito R, Kornetsky C. (1977) Morphine lowering of self-stimulation threshold: Lack of tolerance with long-term administration. Science, 195:189191.

- Erb S, Shaham Y, Stewart J. (1996). Stress reinstates cocaine-seeking behavior after prolonged extinction and a drug-free period. Psychopharmacology, $128: 408-412$.

- Estrada-Camarena E, Fernandez-Guasti A, Lopez-Rubalcava C. (2003). Antidepressant-Like Effect of Different Estrogenic Compounds in the Forced Swimming Test. Neuropsychopharmacology, 28:830-838.

- Fantegrossi W, Kugle KM, Valdes LJ 3rd, Koreeda M, Woods JH (2005). Kappa-opioid receptor-mediated effects of the plant-derived hallucinogen, 
salvinorin A, on inverted screen performance in the mouse. Behav Pharmacol $16: 627-633$.

- Feltenstein MW, See RE. (2008). The neurocircuitry of addiction: an overview. Br J Pharmacol, 154:261-74.

- Fenu S, Rivas E, Di Chiara G (2005). Differential role of dopamine in drug- and lithium-conditioned saccharin avoidance. Physiol Behav 85:37-43.

- Ferragud A, Velázquez-Sánchez C, Hernández-Rabaza V, Nácher A, Merino V, Cardá M, Murga J, Canales JJ. (2009). A dopamine transport inhibitor with markedly low abuse liability suppresses cocaine self-administration in the rat. Psychopharmacology, 207:281-9.

- Frankowska M, Nowak E, Filip M (2009). Effects of GABA B receptor agonists on cocaine hyperlocomotor and sensitizing effects in rats. Pharmacol Rep 61:1042-9.

- Fuchs RA, Tran-Nguyen LTL, Specio SE, Groff RS, Neisewander JL (1998). Predictive validity of the extinction/reinstatement model of drug craving. Psychopharmacology, 135:151-160.

- Funada M, Suzuki T, Narita M, Misawa M, Nagase H. (1993).Blockade of morphine reward through the activation of kappa-opioid receptors in mice. Neuropharmacology, 32:1315-23.

- Fuentealba JA, Gysling K, Magendzo K, Andrés ME. (2006). Repeated administration of the selective kappa-opioid receptor agonist U-69593 increases stimulated dopamine extracellular levels in the rat nucleus accumbens. $J$ Neurosci Res, 84:450-9.

- Garcia J, Kimeldorf DJ, Koelling RA. (1955). Conditioned aversion to saccharin resulting from exposure to gamma radiation. Science, 157-158. 
- Gatch MB, Taylor CM, Flores E, Selvig M, Forster MJ. (2006). Effects of monoamine oxidase inhibitors on cocaine discrimination in rats. Behav Pharmacol, 17:151-9.

- Gawin F, Kleber HD. (1988). Evolving Conceptualizations of Cocaine Dependence. Yale J Biol Med, 61 123-136.

- Gawin F. (1991). Cocaine addiction: psychology and neurophysiology. Science, 251:1580-1586.

- Geer E, Landman R, Wardlaw S, Conwell I, Freda P. (2005). Stimulation of the Hypothalamic-Pituitary-Adrenal Axis with the Opioid Antagonist Nalmefene. Pituitary, 8:115-122.

- Gehrke B, Chefer V, Shippenberg TS (2008). Effects of acute and repeated administration of salvinorin A on dopamine function in the rat dorsal striatum. Psychopharmacology 197:509-517.

- Glick S, Maisonneuve I, Raucci J, Archer S. (1995). Kappa opioid inhibition of morphine and cocaine self-administration in rats. Brain Res 681:147-152.

- Glick SD, Visker KE, Maisonneuve IM. (1998). Effects of cyclazocine on cocaine self-administration in rats. Eur J Pharmacol, 357:9-14.

- Goeders N, Smith J. (1983). Cortical dopaminergic involvement in cocaine reinforcement. Science, 221:773-775.

- Goeders NE. (2002). Stress and Cocaine Addiction. J Pharmacol Exp Ther, 301:785-789.

- Goeders NE. (2002). The HPA axis and cocaine reinforcement. Psychoneuroendocrinology, 27:13-33.

- Goldstein A, Ghazarossian VE. (1980). Immunoreactive dynorphin in pituitary and brain. Proc Natl Acad Sci U S A, 77:6207-6210. 
- Goldstein A, Tachibana S, Lowney L, Hunkapiller M, Hood L. (1979). Dynorphin-(1-13), an extraordinarily potent opioid peptide. Proc Natl Acad Sci $U S A, 76: 6666-6670$.

- Gorelick DA, Gardner EL, Xi ZX. Agents in development for the management of cocaine abuse. Drugs. 2004, 64:1547-73.

- Grabowski J, Shearer J, Merrill J, Negus SS. (2004). Agonist-like, replacement pharmacotherapy for stimulant abuse and dependence. Addict Behav, 29:1439-64.

- Grant JE, Potenza MN, Hollander E, Cunningham-Williams R, Nurminen T, Smits G, et al. (2006). Multicenter Investigation of the Opioid Antagonist Nalmefene in the Treatment of Pathological Gambling. Am J Psychiatry, $163: 303-312$.

- Grant JE, Odlaug BL, Potenza MN, Hollander E, Kim SW. (2010). Nalmefene in the treatment of pathological gambling: multicentre, double-blind, placebocontrolled study. Br J Psychiatry, 197:330-331.

- Grant S, Contoreggi C, London ED. (2000). Drug abusers show impaired performance in a laboratory test of decision making. Neuropsychologia, 38:1180-7.

- Griffin OH, Miller BL, Khey DN. (2008). Legally high? Legal considerations of Salvia divinorum. J Psychoactive Drugs, 40:183-91.

- Grimm JW, Hope BT, Wise RA, Shaham Y. (2001). Neuroadaptation: Incubation of cocaine craving after withdrawal. Nature, 412:141-142.

- Groer CE, Tidgewell K, Moyer RA, Harding WW, Rothman RB, Prisinzano TE, et al. (2007). An Opioid Agonist that Does Not Induce Mu-Opioid Receptor Beta-Arrestin Interactions or Receptor Internalization. Mol Pharmacol, 71:549557. 
- Gunnell D, Irvine D, Wise L, Davies C, Martin RM.(2009). Varenicline and suicidal behaviour: a cohort study based on data from the General Practice Research Database. BMJ. 339:b3805. doi: 10.1136/bmj.b3805.

- Hahn B, Stolerman IP, Shoaib M. (2000). Kappa-opioid receptor modulation of nicotine-induced behaviour. Neuropharmacology;39:2848-55.

- Harding WW, Schmidt M, Tidgewell K, Kannan P, Holden KG, Dersch CM, et al. (2006). Synthetic studies of neoclerodane diterpenes from Salvia divinorum: Selective modification of the furan ring. Bioorg Med Chem Lett, 16:3170-3174.

- Harding WW, Tidgewell K, Byrd N, Cobb H, Dersch CM, Butelman ER, et al. (2005). Neoclerodane Diterpenes as a Novel Scaffold for mu-Opioid Receptor Ligands. J Med Chem, 48:4765-4771.

- Harris LS, Pierson AK. (1964). Some narcotic antagonists in the benzomorphan series. J Pharmacol Exp Ther, 143:141-148.

- Hasebe K, Kawai K, Suzuki T, Kawamura K, Tanaka T, Narita M, Nagase H, Suzuki T. (2004). Possible pharmacotherapy of the opioid kappa receptor agonist for drug dependence. Ann N Y Acad Sci, 1025:404-413.

- Heidbreder C. (2008). Selective antagonism at dopamine D3 receptors as a target for drug addiction pharmacotherapy: a review of preclinical evidence. CNS Neurol Disord Drug Targets, 7:410-21.

- Heidbreder CA, Goldberg SR, Shippenberg TS (1993). The kappa-opioid receptor agonist U69593 attenuates cocaine-induced behavioral sensitization in the rat. Brain Res 616:335-338.

- Heidbreder CA, Shippenberg TS. (1994). U69593 prevents cocaine sensitization by

- normalizing basal accumbens dopamine. NeuroReport, 5:1797-800. 
- Heidbreder CA, Babovic-Vuksanovic D, Shoaib M, Shippenberg TS (1995). Development of behavioral sensitization to cocaine: influence of kappa opioid receptor agonists. J Pharmacol Exp Ther 275:150-163.

- Heidbreder CA, Thompson AC, Shippenberg TS. (1996). Role of extracellular dopamine in the initiation and long-term expression of behavioral sensitization to cocaine. J Pharmacol Exp Ther, 278: 490-502.

- Heinz A, Mann K, Weinberger DR, Goldman D. (2001). Serotonergic Dysfunction, Negative Mood States, and Response to Alcohol. Alcohol Clin Exp Res, 25: 487-495.

- Hickman M, Madden P, Henry J, Baker A, Wallace C, Wakefield J, et al. (2003). Trends in drug overdose deaths in England and Wales 1993-98: methadone does not kill more people than heroin. Addiction, 98:419-25.

- Hill SY, Powell BJ. (1976). Cocaine and morphine self-administration: effects of differential rearing. Pharmacol Biochem Behav, 5:701-4.

- Holz GI, Rane SG, and Dunlap K. (1986). GTP-binding proteins mediate transmitter inhibition of voltage-dependent calcium channels. Nature, 319: 670672.

- Hooker JM, Xu Y, Schiffer W, Shea C, Carter P, Fowler JS (2008). Pharmacokinetics of the potent hallucinogen, salvinorin A in primates parallels the rapid onset and short duration of effects in humans. NeuroImage 41:10441050.

- Hooker J, Patel V, Kothari S, Schiffer W (2009). Metabolic changes in the rodent brain after acute administration of salvinorin A. Mol Imaging Biol $11: 137-143$. 
- Hooks S, Duffy P, Striplin C, Kalivas P (1994). Behavioral and neurochemical sensitization following cocaine self-administration. Psychopharmacology $115: 265-272$.

- Hunt W, Barnett LW, Branch LG. (1971). Relapse rates in addiction programs. $J$ Clin Psychol, 27, 455-456.

- Hymen, S., Malenka, RC (2001). Addiction and the brain: the neurobiology of compulsion and its persistence. Nat Rev, 2, 695-703.

- Ingman K, Hagelberg N, Aalto S, Nagren K, Juhakoski A, Karhuvaara S, et al. (2005). Prolonged Central Mu-Opioid Receptor Occupancy after Single and Repeated Nalmefene Dosing. Neuropsychopharmacology, 30:2245-2253.

- Jaffe JH, Cascella NG, Kumor KM, Sherer MA. (1989). Cocaine-induced cocaine craving. Psychopharmacology, 97:59-64.

- Jorenby DE, Hays JT, Rigotti NA, Azoulay S, Watsky EJ, Williams KE, Billing CB, Gong J, Reeves KR; Varenicline Phase 3 Study Group. (2006). Efficacy of varenicline, an alpha4beta2 nicotinic acetylcholine receptor partial agonist, vs placebo or sustained-release bupropion for smoking cessation: a randomized controlled trial. JAMA, 296:56-63.

- Javitch JA, D'Amato RJ, Strittmatter SM, Snyder SH. (1985). Parkinsonisminducing neurotoxin, N-methyl-4-phenyl-1,2,3,6 -tetrahydropyridine: uptake of the metabolite N-methyl-4-phenylpyridine by dopamine neurons explains selective toxicity. Proc Natl Acad Sci USA, 82: 2173-2177.

- John TF, French LG, Erlichman JS (2006). The antinociceptive effect of salvinorin A in mice. Eur J Pharmacol 545:129-133.

- Johnson MW, MacLean KA, Reissig CJ, Prisinzano TE, Griffiths RR. (2011). Human psychopharmacology and dose-effects of salvinorin A, a kappa opioid 
agonist hallucinogen present in the plant Salvia divinorum. Drug Alcohol Depend, 115: 150-155.

- June H, Grey C, Warren-Reese C, Lawrence A, Thomas A, Cummings R, et al. (1998). The opioid receptor antagonist nalmefene reduces responding maintained by alcohol presentation: preclinical studies in alcohol preferring $(\mathrm{P})$ and outbred Wistar rats. Alcoholism: Clin Exp Res 22, 2174-2185.

- June HL, Cummings R, Eiler WJA II, Foster KL, McKay PF, Seyoum R, et al. (2003). Central Opioid Receptors Differentially Regulate the NalmefeneInduced Suppression of Ethanol- and Saccharin-Reinforced Behaviors in Alcohol-Preferring (P) Rats. Neuropsychopharmacology, 29: 285-299.

- Jupp B, Lawrence AJ. (2010). New horizons for therapeutics in drug and alcohol abuse. Pharmacol Ther, 125: 138-168.

- Kalivas PW, Duffy P (1990). Effect of acute and daily cocaine treatment on extracellular dopamine in the nucleus accumbens. Synapse 5:48-58.

- Kalivas PW, Stewart J. (1991). Dopamine transmission in the initiation and expression of drug- and stress-induced sensitization of motor activity. Brain Res Rev ,16:223-244.

- Kalivas PW, Pierce RC, Cornish J, Sorg BA (1998). A role for sensitization in craving and relapse in cocaine addiction. J Psychopharmacol 12:49-53.

- Kalivas PW, Duffy P, DuMars LA, Skinner C. (1988). Behavioral and neurochemical effects of acute and daily cocaine administration in rats. $J$. Pharmacol. Exp. Ther, 245:485-492.

- Kalivas, P. W., Peters, J., \& Knackstedt, L. (2006). Animal Models and Brain Circuits in Drug Addiction. Mol Interv, 6: 339-344.

- Kampman KM, Dackis C, Lynch KG, Pettinati H, Tirado C, Gariti P, Sparkman T, Atzram M, O'Brien CP. (2006). A double-blind, placebo-controlled trial of 
amantadine, propranolol, and their combination for the treatment of cocaine dependence in patients with severe cocaine withdrawal symptoms. Drug Alcohol Depend, 85:129-37.

- Kantak, K. M., Riberdy, A., \& Spealman, R. D. (1999). Cocaine-opioid interactions in groups of rats trained to discriminate different doses of cocaine. Psychopharmacology, 147: 257-265.

- Karhuvaara S, Simojoki K, Virta A, Rosberg M, Löyttyniemi E, Nurminen T, et al. (2007). Targeted Nalmefene With Simple Medical Management in the Treatment of Heavy Drinkers: A Randomized Double-Blind Placebo-Controlled Multicenter Study. Alcoholism: Clin Exp Res, 31:1179-1187.

- Karila L, Gorelick D, Weinstein A, Noble F, Benyamina A, Coscas S, Blecha L, Lowenstein W, Martinot JL, Reynaud M, Lépine JP. (2008). New treatments for cocaine dependence: a focused review. Int $J$ Neuropsychopharmacol, 11:425-38.

- Keiflin R, Isingrini E, Cador M. (2008). Cocaine-induced reinstatement in rats: evidence for a critical role of cocaine stimulus properties. Psychopharmacology, 197: 649-660. Face value of rein model.

- Kelly BC. (2011). Legally tripping: a qualitative profile of Salvia divinorum use among young adults. $J$ Psychoactive Drugs, 43:46-54.

- Killinger BA, Peet MM, Baker LE. (2010). Salvinorin A fails to substitute for the discriminative stimulus effects of LSD or ketamine in Sprague-Dawley rats. Pharmacology Biochemistry and Behavior, 96: 260-265.

- Kim JA, Pollak KA, Hjelmstad GO, Fields HL. (2004). A single cocaine exposure enhances both opioid reward and aversion through a ventral tegmental area-dependent mechanism. Proc Natl Acad Sci USA, 101: 5664-5669. 
- Kitson TM. (1977). The disulfiram-ethanol reaction: a review. J Stud Alcohol, 38:96-113.

- Kitson TM, Bieder L.(1977). Drugs which interfere with alcohol metabolism. $N$ Z Med J. 86:135-7.

- Kong, H., Raynor, K., Yano, H., Takeda, J., Bell, G. I., \& Reisine, T. (1994). Agonists and antagonists bind to different domains of the cloned kappa opioid receptor. Proc Natl Acad Sci USA, 91: 8042-8046.

- Koob G, Bloom FE. (1988). Cellular and Molecular Mechanisms of Drug Dependence. Science, 242: 715-723.

- Koob GF. (1992). Drugs of abuse: anatomy, pharmacology and function of reward pathways. Trends Pharmacol Sci, 13, 177-184.

- Koob G, Weiss F, Deroche V, Weissenborn R. (1996). Effects of dopamine agonists and antagonists on cocaine-induced operant responding for a cocaineassociated stimulus. Psychopharmacology, 126: 311-322.

- Koob GF, Le Moal M. (1997). Drug Abuse: Hedonic Homeostatic Dysregulation. Science, 278: 52-58.

- Koob GF. (2000). Neurobiology of Addiction: Toward the Development of New Therapies. Ann NY Acad Sci, 909:170-185.

- Koob G, Le Moal M. (2001). Drug Addiction, Dysregulation of Reward, and Allostasis. Neuropsychopharmacology, 24, 97-129.

- Koob GF, Ahmed SH, Boutrel B, Chen SA, Kenny PJ, Markou A, et al. (2004). Neurobiological mechanisms in the transition from drug use to drug dependence. Neurosci Biobehav Rev, 27: 739-749.

- Koob G, La Moal M. (Ed.). (2005). Neurobiology of Addiction: Elsvier (Academic Press). 
- Koob GF, Le Moal M. (2008). Neurobiological mechanisms for opponent motivational processes in addiction. Phil Trans Royal Soc B Biol Sci, 363:31133123.

- Koob GF, Volkow ND. (2009). Neurocircuitry of Addiction. Neuropsychopharmacology, 35: 217-238.

- Kornetsky C, Esposito RU. (1981). Reward and detection thresholds for brain stimulation: dissociative effects of cocaine. Brain Res, 209:496-500.

- Kreek MJ, Bart G, Lilly C, Laforge KS, Nielsen DA. (2005). Pharmacogenetics and Human Molecular Genetics of Opiate and Cocaine Addictions and Their Treatments. Pharmacol Rev, 57: 1-26.

- Kreek MJ, LaForge KS, Butelman E. (2002). Pharmacotherapy of addictions. Nat Rev Drug Discov, 1(9), 710-726.

- Kreek MJ, Schluger J, Borg L, Gunduz M, Ho A. (1999). Dynorphin A (1-13) Causes Elevation of Serum Levels of Prolactin Through an Opioid Receptor Mechanism in Humans: Gender Differences and Implications for Modulation of Dopaminergic Tone in the Treatment of Addictions. J Pharmacol Exp Ther, 288: 260-269.

- Kreek MJ. (2000). Methadone-related opioid agonist pharmacotherapy for heroin addiction. History, recent molecular and neurochemical research and future in mainstream medicine. Ann N Y Acad Sci, 909:186-216.

- Kreibich AS, Blendy JA. (2004). cAMP Response Element-Binding Protein Is Required for Stress But Not Cocaine-Induced Reinstatement. J Neurosci, 24(30), 6686-6692.

- Kuhar MJ, Ritz MC, Boja JW. (1991). The dopamine hypothesis of the reinforcing properties of cocaine. Trends Neurosci, 14: 299-302. 
- Kupferschmidt DA, Brown ZJ, Erb S. (2010). A Procedure for Studying the Footshock-Induced Reinstatement of Cocaine Seeking in Laboratory Rats. J Vis Exp, 47: e2265.

- Kuzmin AV, Semenova S, Gerrits MA, Zvartau EE, Van Ree JM (1997). Kappa-opioid receptor agonist U50488H modulates cocaine and morphine selfadministration in drug-naive rats and mice. Eur J Pharmacol 321:265-271.

- Lahti RA, Mickelson MM, McCall JM, Von Voigtlander PF. (1985). [3H]U69593 a highly selective ligand for the kappa-opioid receptor. Eur J Pharmacol, 109:281-284.

- Lahti RA, Vonvoigtlander PF, Barsuhn C. (1982). Properties of a selective kappa-opioid agonist, U-50,488H. Life Sci, 31:2257-2260.

- Land BB, Bruchas MR, Schattauer S, Giardino WJ, Aita M, Messinger D, et al. (2009). Activation of the kappa opioid receptor in the dorsal raphe nucleus mediates the aversive effects of stress and reinstates drug seeking. Proc Natl Acad Sci, 106:19168-19173.

- Lambert NM, McLeod M, Schenk S. (2006). Subjective responses to initial experience with cocaine: an exploration of the incentive-sensitization theory of drug abuse. Addiction, 101: 713-725.

- Li S, Zhu J, Chen C, Chen Y, Deriel J, Ashby B, Liu-Chen LY. (1993). Molecular cloning and expression of a rat kappa opioid receptor. Biochem J. , 295:629-633.

- Liang J, Li Y, Ping X, Yu P, Zuo Y, Wu L, et al. (2006). The possible involvement of endogenous ligands for mu-, delta- and kappa-opioid receptors in modulating morphine-induced CPP expression in rats. Peptides, 27: 33073314. 
- Lin Z, Canales JJ, Björgvinsson T, Thomsen M, Qu H, Liu QR, Torres GE, Caine SB. (2011). Monoamine transporters: vulnerable and vital doorkeepers. Prog Mol Biol Transl Sci, 98:1-46.

- Littleton J. (2000). Can craving be modeled in animals? The relapse prevention perspective. Addiction., 95:S83-S90.

- Lobmaier P, Gossop M, Waal H, Bramness J. (2010). The pharmacological treatment of opioid addiction-a clinical perspective. Eur J Clin Pharmacol, $66: 537-45$.

- Lu, Z., 2004. Mechanism of rectification in inward-rectifier K+channels. Annu. Rev. Physiol. 66, 103-129.

- Ludwig AM, Wikler A, Stark LH. (1974). The First Drink: Psychobiological Aspects of Craving. Arch Gen Psychiatry, 30:539-547.

- Mague SD, Pliakas AM, Todtenkopf MS, Tomasiewicz HC, Zhang Y, Stevens WC, et al. (2003). Antidepressant-Like Effects of $\kappa$-Opioid Receptor Antagonists in the Forced Swim Test in Rats. J Pharmacol Exp Ther, 305: 323330.

- Maisonneuve IM, Archer S, Glick SD. (1994). U50488, a kappa-opioid receptor agonist, attenuates cocaine-induced increases in extracellular dopamine in the nucleus accumbens of rats. Neurosci Lett, 181:57-60.

- Maisonneuve IM, Glick SD. (2003). Anti-addictive actions of an iboga alkaloid congener: a novel mechanism for a novel treatment. Pharmacol Biochem Behav, 75:607-618.

- Malagié I, Trillat AC, Jacquot C, Gardier AM. (1995). Effects of acute fluoxetine on extracellular serotonin levels in the raphe: an in vivo microdialysis study. Eur J Pharmacol, 286:213-217. 
- Mansour A, Hoversten MT, Taylor LP, Watson SJ, Akil H. (1995). The cloned mu, delta and kappa receptors and their endogenous ligands: Evidence for two opioid peptide recognition cores. Brain Res, 700: 89-98.

- Mansour A, Burke S, Pavlic RJ, Akil H, Watson SJ. (1996). Immunohistochemical localization of the cloned kappa1-receptor in the rat CNS and pituitary. Neuroscience, 71: 671-690.

- Margolis EB, Hjelmstad GO, Bonci A, Fields HL. (2003). א-Opioid Agonists Directly Inhibit Midbrain Dopaminergic Neurons. J Neurosci, 23:9981-9986.

- Margolis EB, Lock H, Chefer VI, Shippenberg TS, Hjelmstad GO, Fields HL. (2006). Kappa- opioids selectively control dopaminergic neurons projecting to the prefrontal cortex. Proc Natl Acad Sci USA, 103: 2938-2942.

- Markou A, Koob GF. (1991). Post-cocaine anhedonia - an animal model of cocaine withdrawal. Neuropsychopharmacology, 4:17-26.

- Markou A, Weiss F, Gold L, Caine S, Schulteis G, Koob G. (1993). Animal models of drug craving. Psychopharmacology, 112:163-182.

- Mason BJ, Salvato FR, Williams LD, Ritvo EC, Cutler RB. (1999). A Doubleblind, Placebo-Controlled Study of Oral Nalmefene for Alcohol Dependence. Arch Gen Psychiatry, 56:719-724.

- Mason JN, Farmer H, Tomlinson ID, Schwartz JW, Savchenko V, DeFelice LJ, Rosenthal SJ, Blakely RD. (2005). Novel fluorescence-based approaches for the study of biogenic amine transporter localization, activity, and regulation. $\mathrm{J}$ Neurosci Methods., 143:3-25.

- Mattick RP, Kimber J, Breen C, Davoli M. (2008). Buprenorphine maintenance versus placebo or methadone maintenance for opioid dependence. Cochrane Database of Systematic Reviews 2008, Issue 2. Art. No.: CD002207. doi:10.1002/14651858.CD002207.pub3. 
- McCurdy CR, Sufka KJ, Smith GH, Warnick JE, Nieto MJ (2006). Antinociceptive profile of salvinorin A, a structurally unique kappa opioid receptor agonist. Pharmacol Biochem Behav 83:109-113.

- McFarland K, Ettenberg A. (1997). Reinstatement of drug-seeking behavior produced by heroin-predictive environmental stimuli. Psychopharmacology, 131:86-92.

- McFarland K, Kalivas PW. (2001). The Circuitry Mediating Cocaine-Induced Reinstatement of Drug-Seeking Behavior. J. Neurosci, 21:8655-8663.

- McLaughlin JP, Marton-Popovici M, Chavkin C. (2003). Kappa-Opioid Receptor Antagonism and Prodynorphin Gene Disruption Block Stress-Induced Behavioral Responses. J Neurosci, 23:5674-5683.

- McLaughlin JP, Land BB, Li S, Pintar JE, Chavkin C. (2005). Prior Activation of Kappa Opioid Receptors by U50,488 Mimics Repeated Forced Swim Stress to Potentiate Cocaine Place Preference Conditioning. Neuropsychopharmacology, 31:787-794.

- McLaughlin JP, Li S, Valdez J, Chavkin TA, Chavkin C. (2005). Social Defeat Stress-Induced Behavioral Responses are Mediated by the Endogenous Kappa Opioid System. Neuropsychopharmacology, 31:1241-1248.

- McLellan AT, Lewis DC, O'Brien CP, Kleber HD. (2004). Drug dependence, a chronic medical illness: Implications for treatment, insurance, and outcomes evaluation. JAMA, 284:1689-1695.

- McLennan GP, Kiss A, Miyatake M, Belcheva MM, Chambers KT, Pozek JJ, Mohabbat Y, Moyer RA, Bohn LM, Coscia CJ. (2008). Kappa opioids promote the proliferation of astrocytes via Gbetagamma and beta-arrestin 2-dependent MAPK-mediated pathways. J Neurochem, 107:1753-65. 
- Meini M, Capovani B, Sbrana A, Massei GJ, Ravani L, Massimetti G, Daini L, Scaramelli D, Moncini M. (2008). A pilot open-label trial of ropinirole for cocaine dependence. Am J Addict, 17:165-6.

- Mello NK, NEGUS SS. (1996). Preclinical evaluation of pharmacotherapies for treatment of cocaine and opioid abuse using drug self-administration procedures.

- Neuropsychopharmacology, 14: 375-424.

- Mello NK, Negus SS. (1998). Effects of Kappa Opioid Agonists on Cocaineand Food-Maintained Responding by Rhesus Monkeys. J Pharmacol Exp Ther, 286: $812-824$.

- Mello NK, Negus SS. (2000). Interactions between Kappa Opioid Agonists and Cocaine: Preclinical Studies. Ann NY Acad Sci, 909: 104-132.

- Meng F, Xie G, Thompson R, Mansour A, Goldstein A, Watson S, et al. (1993). Cloning and pharmacological characterization of a rat kappa opioid receptor. Proc Natl Acad Sci U S A, 90:9954-9958.

- Morani AS, Kivell B, Prisinzano TE, Schenk S (2009). Effect of kappa-opioid receptor agonists U69593, U50488H, spiradoline and salvinorin A on cocaineinduced drug-seeking in rats. Pharmacol Biochem Behav 94:244-249.

- Mori T, Nomura M, Nagase H, Narita M, Suzuki T. (2002). Effects of a newly synthesized kappa-opioid receptor agonist, TRK-820, on the discriminative stimulus and rewarding effects of cocaine in rats. Psychopharmacology, 161:1722.

- Morton, A. (1999). Cocaine and Psychiatric Symptoms. Prim Care Companion J Clin Psychiatry, 1:109-113.

- Mowry M, Mosher M, Briner W. (2003). Acute physiologic and chronic histologic changes in rats and mice exposed to the unique hallucinogen salvinorin A. J. Psychoactive Drugs, 35:379-382. 
- Mucha RF, Herz A (1985). Motivational properties of kappa and mu opioid receptor agonists studied with place and taste preference conditioning. Psychopharmacology 86:274-280.

- Mucha RF, Herz A. (1986). Preference conditioning produced by opioid active and inactive isomers of levorphanol and morphine in rat. Life Sci, 38:241-249.

- Mueller D, Stewart J. (2000). Cocaine-induced conditioned place preference: reinstatement by priming injections of cocaine after extinction. Behav Brain Res, 115:39-47.

- Munro TA, Duncan KK, Xu W, Wang Y, Liu-Chen LY, Carlezon WA., et al. (2008). Standard protecting groups create potent and selective kappa-opioids: Salvinorin B alkoxymethyl ethers. Bioorg Med Chem, 16:1279-1286.

- Murrill CS, Prevots DR, Miller MS, Linley LA, Royalty JE, Gwinn M, Seroincidence Study Group. (2001). Incidence of HIV among injection drug users entering drug treatment programs in four US cities. J Urban Health. , 78:152-161.

- Muscat R, Willner P. (1992). Suppression of sucrose drinking by chronic mild unpredictable stress: a methodological analysis. Neurosci Biobehav Rev, 16:507-17.

- Mysels D, Sullivan MA. (2009). The kappa-opiate receptor impacts the pathophysiology and behavior of substance use. Am J Addict, 18: 272-276.

- Nagase H, Hayakawa J, Kawamura K, Kawai K, Takezawa Y, Matsuura H, et al. (1998). Discovery of a structurally novel opioid kappa-agonist derived from 4,5epoxymorphinan. Chem Pharm Bull (Tokyo)46:366-369.

- Negus SS, Mello NK, Portoghese PS, Lin CE. (1997). Effects of kappa opioids on cocaine self-administration by rhesus monkeys. J Pharmacol Exp Ther, 282: $44-55$. 
- Negus SS, Mello NK. (2004). Effects of chronic methadone treatment on cocaine- and food-maintained responding under second-order, progressive-ratio and concurrent-choice schedules in rhesus monkeys. Drug Alcohol Depend, 74:297-309. Negus SS. (2004). Effects of the kappa opioid agonist U50,488 and the kappa opioid antagonist nor-binaltorphimine on choice between cocaine and food in rhesus monkeys. Psychopharmacology, 176:204-13.

- Nemmani KVS, Gullapalli S, Ramarao P. (2001). Potentiation of kappa-opioid receptor agonist-induced analgesia and hypothermia by fluoxetine. Pharmacol Biochem Behav, 69:189-193.

- Nemmani KVS, Mogil JS. (2003). Serotonin-GABA interactions in the modulation of mu- and kappa-opioid analgesia. Neuropharmacology, 44:304310.

- Nestby P, Schoffelmeer ANM, Homberg JR, Wardeh G, De Vries TJ, Mulder $\mathrm{AH}$, et al. (1999). Bremazocine reduces unrestricted free-choice ethanol selfadministration in rats without affecting sucrose preference. Psychopharmacology, 142:309-317.

- Nestler EJ, Aghajanian GK. (1997). Molecular and cellular basis of addiction. Science, 278:58-63.

- Nestler EJ. (2001). Molecular basis of long-term plasticity underlying addiction. Nat Rev Neurosci, 2:119-28.

- Nestler EJ, Carlezon WA. (2006). The mesolimbic dopamine reward circuit in depression. Biol Psychiatry, 59: 1151-1159.

- Neumeyer JL, Gu XH, van Vliet LA, DeNunzio NJ, Rusovici DE, Cohen DJ, et al. (2001). Mixed kappa agonists and mu agonists/antagonists as potential pharmacotherapeutics for cocaine abuse: synthesis and opioid receptor binding 
affinity of N-substituted derivatives of morphinan. Bioorg Med Chem Lett, $11: 2735-2740$.

- Nobre MJ, Ribeiro dos Santos N, Aguiar MS, Brandão ML. (2000). Blockade of mu- and activation of kappa-opioid receptors in the dorsal periaqueductal gray matter produce defensive behavior in rats tested in the elevated plus-maze. Eur $J$ Pharmacol, 404:145-151.

- O'Brien CP, Childress AR, McLellan AT, Ehrman R. (1992). Classical Conditioning in Drug-Dependent Humans. Ann NY Acad Sci, 654:400-415.

- O'Brien CP, Childress AR, Ehrman R, Robbins SJ. (1998). Conditioning factors in drug abuse: can they explain compulsion? J Psychopharmacol, 12:15-22.

- O'Brien CP, Gardner EL. (2005). Critical assessment of how to study addiction and its treatment: Human and non-human animal models. Pharmacol Ther, 108:18-58.

- Oka T, Hosoya E. (1976). Effect of humoral modulators of morphine-induced increase in locomotor activity of mice. Jpn J Pharmacol, 26:615-9.

- Olds J, Milner P. (1954). Positive reinforcement produced by electrical stimulation of the septal area and other regions of rat brain. J Comp Physiol Psychol, 47:419-427.

- Olds J. (1956). Pleasure centers in the brain. Scientific American.

- Olds J. (1977). Drives and reinforcements: Behavioral Studies of Hypothalamic Functions. New York Raven Press.

- Olmstead M. (2006). Animal models of drug addiction: Where do we go from here? Q J Exp Psychol (Colchester), 59:625-653.

- Osborn MD, Lowery JJ, Skorput AGJ, Giuvelis D, Bilsky EJ. (2010). In vivo characterization of the opioid antagonist nalmefene in mice. Life Sci, 86:624-630. 
- Ott, J. (1995). Ethnopharmacognosy and human pharmacology of Salvia divinorum and salvinorin A. Curare, 18:103-129.

- Owens MJ, Nemeroff CB. (1994). Role of serotonin in the pathophysiology of depression: focus on the serotonin transporter. Clin Chem. 1994 Feb;40(2):28895.

- Owens MJ, Knight DL, Nemeroff CB. (2001). Second-generation SSRIs: human monoamine transporter binding profile of escitalopram and R-fluoxetine. Biol Psychiatry, 50:345-50.n

- Oz M, Libby T, Kivell B, Jaligam V, Ramamoorthy S, Shippenberg TS. (2010). Real-time, spatially resolved analysis of serotonin transporter activity and regulation using the fluorescent substrate, $\mathrm{ASP}^{+} . J$ Neurochem, 114:1019-1029.

- Pan, Z. Z. (1998). Mu-opposing actions of the kappa-opioid receptor. Trends Pharmacol Sci, 19: 94-98.

- Pare CM. (1971). Monamine oxidase inhibitors and brain monamines in clinical conditions. Biochem J,121:36P-37P.

- Pare CM, Trenchard A, Turner P. (1974). 5-Hydroxytryptamine in depression. Adv Biochem Psychopharmacol, 11:275-9.

- Paulzen M, Gründer G. (2008). Toxic psychosis after intake of the hallucinogen salvinorin A. J. Clin. Psychiatry, 69:1501-1502.

- Paulus I, Halliday R. (1967). Rehabilitation and the narcotic addict: results of a comparative methadone withdrawal program. Can Med Assoc J, 96:655-659. Pavlov IP. (1927). Conditioned reflexes. Oxford University Press, Oxford.

- Perret G, Déglon JJ, Kreek MJ, Ho A, La Harpe R. (2000). Lethal methadone intoxications in Geneva, Switzerland, from 1994 to 1998. Addiction, 95:1647-53.

- Phillips A, Ciano P (1996). Behavioral sensitization is induced by intravenous self-administration of cocaine by rats. Psychopharmacology 124:279-281. 
- Pfeiffer A, Brantl V, Herz A, Emrich HM. (1986). Psychotomimesis mediated by kappa opiate receptors. Science, 233:774-6.

- Pickens R, Thompson T. (1968). Cocaine-reinforced behaviour in rats: Effects of reinforcement magnitude and fixed-ratio size. J Pharmacol Exp Ther, 161:122-129.

- Pierce CR, Kaliwas PW. (1997). A circuitry model of the expression of behavioral sensitization to amphetamine-like psychostimulants. Brain Research Reviews, 25:192-216.

- Pinnock RD. (1992). Activation of kappa-opioid receptors depresses electrically evoked excitatory postsynaptic potentials on 5-HT-sensitive neurones in the rat dorsal raphé nucleus in vitro. Brain Res 583: 237-246.

- Pliakas AM, Carlson RR, Neve RL, Konradi C, Nestler EJ, Carlezon WA Jr. (2001). Altered responsiveness to cocaine and increased immobility in the forced swim test associated with elevated cAMP response element-binding protein expression in nucleus accumbens. J Neurosci 21:7397-403.

- Portoghese PS, Lipkowski AW, Takemori AE. (1987). Binaltorphimine and norbinaltorphimine, potent and selective ${ }_{k}$-opioid receptor antagonists. Life Sci 40:1287-1292.

- Porsolt R (1979). Animal model of depression. Biomedicine 30:139-140.

- Post R, Lockfeldk A, Squjllac M, Contel N. (1981). Drug-environment interaction: Context dependency of cocaine-induced behavioral sensitization. Life Sci., 28, 755-760.

- Post R, Contel N. (1983). Human and animal studies of cocaine: Implications for development of behavioral pathology. Stimulants: Neurochemical, Behavioral, and Clinical Perspective. Raven Press. New York, Edition 1, 169-203. 
- Post R, Weiss SR, Pert A, Uhde T (1987). Chronic cocaine administration: Sensitization and kindling effects. In: Cocaine: Clinical and biobehavioral aspects. Fisher S, Raskin A, Uhlenhuth EH (editors). New York: Oxford University Press; pp. 109-173.

- Preston K, Umbricht A, Schroeder J, Abreu M, Epstein D, Pickworth W. (2004). Cyclazocine: comparison to hydromorphone and interaction with cocaine. Behav Pharmacol, 15:91-102.

- Prevatt-Smith KM, Prisinzano TE. (2010). New therapeutic potential for psychoactive natural products. Nat Prod Rep, 27: 23-31.

- Prisinzano TE. (2005). Psychopharmacology of the hallucinogenic sage Salvia divinorum. Life Sciences, 78: 527-531.

- Prisinzano T, Tidgewell K, Harding WW. (2005). Kappa opioids as potential treatments for stimulant dependence. AAPS J 7:E592-599.

- Prisinzano TE, Rothman RB (2008). Salvinorin A analogs as probes in opioid pharmacology. Chem Rev 108:1732-1743.

- Prisinzano TE. (2008). Natural Products as Tools for Neuroscience: Discovery and Development of Novel Agents to Treat Drug Abuse. J Nat Products, 72: $581-587$

- Przekop P, Lee T. (2009). Persistent psychosis associated with Salvia divinorum use. Am. J. Psychiatry, $166: 7$.

- Puig-Ramos A, Santiago GS, Segarra AC. (2008). U-69593, a Kappa Opioid Receptor Agonist, Decreases Cocaine-Induced Behavioral Sensitization in Female Rats. Behav Neurosci, 122:151-160.

- Quirion R, Pilapil C, Magnan J. (1987). Localization of kappa opioid receptor binding sites in human forebrain using [3H]U69,593: comparison with [3H]bremazocine. Cell Mol Neurobiol, 7: 303-307. 
- Ramamoorthy S, Bauman AL, Moore KR, Han H, Yang-Feng T, Chang AS, et al. (1993). Antidepressant- and cocaine-sensitive human serotonin transporter: molecular cloning, expression, and chromosomal localization. Proc Natl Acad Sci USA, 90: 2542-2546.

- Ravna AW, Sylte I, Dahl SG. (2003). Molecular mechanism of citalopram and cocaine interactions with neurotransmitter transporters. J Pharmacol Exp Ther. $307: 34-41$.

- Redila V, Chavkin C. (2008). Stress-induced reinstatement of cocaine seeking is mediated by the kappa opioid system. Psychopharmacology, 200: 59-70.

- Reingardiene D, Jodziūniene L, Lazauskas R. (2009). Methadone treatment and its dangers. Medicina (Kaunas), 45:419-425.

- Reith M, Li MY, Yan QS. (1997). Extracellular dopamine, norepinephrine, and serotonin in the ventral tegmental area and nucleus accumbens of freely moving rats during intracerebral dialysis following systemic administration of cocaine and other uptake blockers. Psychopharmacology, 134: 309-317.

- Robinson TE, Berridge KC (1993). The neural basis of drug craving: an incentive-sensitization theory of addiction, Brain Res Rev 18:247-291.

- Robinson TE, Berridge KC (2001). Incentive-sensitization and addiction, Addiction 96:103-114.

- Robinson TE, Berridge KC (2003). Addiction. Annual Review of Psychology, 54: $25-53$.

- Robinson TE, Berridge KC. (2008). The incentive sensitization theory of addiction: some current issues. Phil Trans Royal Soc B Biol Sci, 363:3137-3146.

- Römer D, Büscher H, Hill RC, Maurer R, Petcher TJ, Welle HB, Bakel HC, Akkerman AM. (1980). Bremazocine: a potent, long-acting opiate kappa-agonist. Life Sci, 27:971-8. 
- Romualdi P, Donatini A, Izenwasser S, Cox BM, Ferri S. (1996). Chronic intracerebroventricular cocaine differentially affects prodynorphin gene expression in rat hypothalamus and caudate-putamen. Mol Brain Res, 40:153156.

- Roth BL, Baner K, Westkaemper R, Siebert D, Rice KC, Steinberg S, et al. (2002). Salvinorin A: A potent naturally occurring non-nitrogenous kappaopioid selective agonist. Proc Natl Acad Sci USA 99:11934-11939.

- Rothman RB, Blough BE, Baumann MH. (2008). Dual dopamine/serotonin releasers: potential treatment agents for stimulant addiction. Exp Clin Psychopharmacology, 16:458-74.

- Ronken E, Van Muiswinkel FL, Mulder AH, Schoffelmeer AN. (1993). Opioid receptor-mediated inhibition of evoked catecholamine release from cultured neurons of rat ventral mesencephalon and locus coeruleus. Eur J Pharmacol $230: 349-355$

- Rudnick, G. (2006). Serotonin Transporters - Structure and Functions. J Membrane Biol, 213:101-110.

- Rüedi-Bettschen D, Rowlett J, Spealman R, Platt D. (2010). Attenuation of cocaine-induced reinstatement of drug seeking in squirrel monkeys: kappa opioid and serotonergic mechanisms. Psychopharmacology, 210:169-177.

- Saier MH. (1999). A functional-phylogenetic system for the classification of transport proteins. J Cell Biochem, 75: 84-94.

- Salamon Z, Hruby VJ, Tollin G, Cowell S. (2002). Binding of agonists antagonists and inverse agonists to the human delta-opioid receptor produces distinctly different conformational states distinguishable by plasmon-waveguide resonance spectroscopy. J Pept Res, 60:322-328. 
- Sams-Dodd, F (1997). Effect of novel antipsychotic drugs on phencyclidineinduced stereotyped behaviour and social isolation in the rat social interaction test. Behav Pharmacol, 8:196-215.

- Sanchis-Segura C, Spanagel R. (2006). Behavioural assessment of drug reinforcement and addictive features in rodents: an overview. Addict Biol, 11:238.

- Sante AB, Nobre MJ, Brand ÃML. (2000). Place aversion induced by blockade of mu or activation of kappa opioid receptors in the dorsal periaqueductal gray matter. Behav Pharmacol, 11: 583-589.

- Schenk S, Hunt T, Klukowski G, Amit Z (1987). Isolation housing decreases the effectiveness of morphine in the conditioned taste aversion paradigm. Psychopharmacology (Berl) 92:48-51.

- Schenk S, Partridge B. (1997). Sensitization and Tolerance in Psychostimulant Self-Administration. Pharmacol Biochem Behav, 57:543-550.

- Schenk S, Partridge B. (1999). Cocaine-seeking produced by experimenteradministered drug injections: dose-effect relationships in rats. Psychopharmacology, 147: 285-290.

- Schenk S, Partridge B, Shippenberg TS (1999). U69593, a kappa-opioid agonist, decreases cocaine self-administration and decreases cocaine-produced drugseeking. Psychopharmacology 144:339-346.

- Schenk S, Partridge B, Shippenberg TS (2000). Reinstatement of extinguished drug-taking behavior in rats: effect of the kappa-opioid receptor agonist U69593. Psychopharmacology 151:85-90.

- Schenk S, Partridge B. (2000). Sensitization to Cocaine's Reinforcing Effects Produced by Various Cocaine Pretreatment Regimens in Rats. Pharmacol Biochem Behav, 66: 765-770. 
- Schenk S. (2000). Effects of the serotonin 5-HT2 antagonist, ritanserin, and the serotonin 5-HT1A antagonist, WAY 100635, on cocaine-seeking in rats. Pharmacol Biochem Behav, 67: 363-369.

- Schenk S, Partridge B. (2001). Influence of a conditioned light stimulus on cocaine self-administration in rats. Psychopharmacology, 154: 390-396.

- Schenk, S., Partridge B, Shippenberg TS. (2001). Effects of the kappa-opioid receptor agonist, U69593, on the development of sensitization and on the maintenance of cocaine self-administration. Neuropsychopharmacology, 24:441450.

- Schenk S, Partridge B. (2001). Effect of the kappa-opioid receptor agonist, U69593, on reinstatement of extinguished amphetamine self-administration behavior. Pharmacol Biochem Behav, 68: 629-634.

- Schenk S, Harper DN, Do J. (2010). Novel object recognition memory: measurement issues and effects of MDMA self-administration following short inter-trial intervals. J Psychopharmacol, DOI: 10.1177/0269881110389213

- Schmidt, H. D, Anderson SM, Famous KR, Kumaresan V, Pierce RC. (2005). Anatomy and pharmacology of cocaine priming-induced reinstatement of drug seeking. Eur J Pharmacol, 526:65-76.

- Schmidt MD, Schmidt MS, Butelman ER, Harding WW, Tidgewell K, Murry DJ, et al. (2005). Pharmacokinetics of the plant-derived kappa-opioid hallucinogen salvinorin A in nonhuman primates. Synapse 58:208-210.

- Schneider M, Schömig E, Leweke FM. (2008). Acute and chronic cannabinoid treatment differentially affects recognition memory and social behavior in pubertal and adult rats. Addict Biol, 13:345-57.

- Schwartz JW, Blakely RD, DeFelice LJ. (2003). Binding and Transport in Norepinephrine Transporters. J Biol Chem, 278: 9768-9777. 
- Schwartz JW, Novarino G, Piston DW, DeFelice LJ. (2005). Substrate binding stoichiometry and kinetics of the norepinephrine transporter. J Biol Chem., 280:19177-84.

- Self DW. (2004). Regulation of drug-taking and -seeking behaviors by neuroadaptations in the mesolimbic dopamine system. Neuropharmacology, $47: 242-255$.

- Self DW, Choi KH, Simmons D, Walker JR, Smagula CS. (2004). Extinction training regulates neuroadaptive responses to withdrawal from chronic cocaine self-administration. Learn Mem. 11:648-57.

- Sershen H, Hashim A, Harsing L, Lajtha A. (1992). Ibogaine antagonizes cocaine-induced locomotor stimulation in mice. Life Sci. 50:1079-1086.

- Shaham Y, Erb S, Stewart J. (2000). Stress-induced relapse to heroin and cocaine seeking in rats: a review. Brain Res Rev, 33:13-33.

- Shaham Y, Shalev U, Lu L, de Wit H, Stewart J. (2003). The reinstatement model of drug relapse: history, methodology and major findings. Psychopharmacology, 168: 3-20.

- Shalev U, Grimm JW, Shaham Y. (2002). Neurobiology of Relapse to Heroin and Cocaine Seeking: A Review. Pharmacol Rev, 54:1-42.

- Shippenberg TS, Herz A (1986). Differential effects of mu and kappa opioid systems on motivational processes. NIDA Res Monogr 75:563-566.

- Shippenberg TS, Heidbreder C. (1995). Sensitization to the conditioned rewarding effects of cocaine: pharmacological and temporal characteristics. $J$ Pharmacol Exp Ther, 273: 808-815.

- Shippenberg TS, LeFevour A, Heidbreder C (1996). kappa-Opioid receptor agonists prevent sensitization to the conditioned rewarding effects of cocaine. $J$ Pharmacol Exp Ther 276:545-54. 
- Shippenberg TS, LeFevour A, Thompson AC. (1998). Sensitization to the conditioned rewarding effects of morphine and cocaine: differential effects of the [kappa]-opioid receptor agonist U69593. Eur J Pharmacol, 345: 27-34.

- Shippenberg TS, Chefer VI, Zapata A, Heidbreder CA. (2001). Modulation of the Behavioral and Neurochemical Effects of Psychostimulants by $\kappa$-Opioid Receptor Systems. Ann NY Acad Sci, 937: 50-73.

- Shippenberg TS, Zapata A, Chefer VI (2007). Dynorphin and the pathophysiology of drug addiction. Pharmacol Ther 116:306-321.

- Siebert, D. J. (1994). Salvia divinorum and salvinorin A: new pharmacologic findings. J Ethnopharmacol, 43: 53-56.

- Siegel RK. (1982). Cocaine smoking. J Psychoactive Drugs. 14:271-359. Cocaine use over 3000 years.

- Silagy C, Lancaster T, Stead L,Mant D, Fowler G. (2004). Nicotine replacement therapy for smoking cessation. Cochrane Database Syst Rev, 3: CD000146.

- Singh, S. (2007). Adolescent salvia substance abuse. Addiction, 102: 823-824.

- Sinha R, Catapano D, O’Malley S. (1999). Stress-induced craving and stress response in cocaine dependent individuals. Psychopharmacology, 142:343-351.

- Sinha R. (2001). How does stress increase risk of drug abuse and relapse? Psychopharmacology, 158:343-359.

- Sinha R, Garcia M, Paliwal P, Kreek MJ, Rounsaville BJ. (2006). StressInduced Cocaine Craving and Hypothalamic-Pituitary-Adrenal Responses Are Predictive of Cocaine Relapse Outcomes. Arch Gen Psychiatry, 63: 324-331.

- Sivam SP. (1989). Cocaine selectively increases striatonigral dynorphin levels by a dopaminergic mechanism. J Pharmacol Exp Ther, 250: 818-824.

- Skinner BF. (1938). The behavior of organisms. Appleton-Century-Crofts, New York. 
- Smith JC, Morris DD, Hendricks J (1964). Conditioned aversion to saccharin solution with dose rates of X-rays as the unconditioned stimulus. Radiat Res 22:507-10.

- Smith MA, Gordon KA, Craig CK, Bryant PA, Ferguson ME, French AM, Gray JD, McClean JM, Tetirick JC. (2003). Interactions between opioids and cocaine on locomotor activity in rats: influence of an opioid's relative efficacy at the mu receptor. Psychopharmacology (Berl), 167:265-73.

- Smith MA, Greene-Naples JL, Felder JN, Lordanou JC, Lyle MA, Walker KL. (2009). The Effects of Repeated Opioid Administration on Locomotor Activity: II. Unidirectional Cross-Sensitization to Cocaine. J Pharmacol Exp Ther, 330: 476-486.

- Sonders MS, Zhu SJ, Zahniser NR, Kavanaugh MP, Amara SG. (1997). Multiple ionic conductances of the human dopamine transporter: the actions of dopamine and psychostimulants. $J$ Neurosci.17:960-74.

- Sorensen J, Masson CL, Perlman DC. (2002). HIV/hepatitis prevention in drug abuse treatment programs: guidance from research. Sci Pract Perspect., 1: 4-11.

- Soyka M, Zingg C, Koller G, Kuefner H. (2008). Retention rate and substance use in methadone and buprenorphine maintenance therapy and predictors of outcome: results from a randomized study. Int $J$ Neuropsychopharmacol, 11:641-53.

- Soyka M, Rosner S. (2010). Nalmefene for treatment of alcohol dependence. Expert Opin Invest Drugs, 19: 1451-1459.

- Spanagel R, Herz A, Shippenberg TS. (1990).The effects of opioid peptides on dopamine release in the nucleus accumbens: an in vivo microdialysis study. $J$ Neurochem 55:1734-40. 
- Spanagel R, Herz A, Shippenberg TS. (1992). Opposing tonically active endogenous opioid systems modulate the mesolimbic dopaminergic pathway. Proceedings of the National Academy of Sciences, 89: 2046-2050.

- Spanagel R, Almeida O, Bartl C, Shippenberg T. (1994). Endogenous א-opioid systems in opiate withdrawal: role in aversion and accompanying changes in mesolimbic dopamine release. Psychopharmacology, 115:121-127.

- Spanagel R, Weiss F. (1999). The dopamine hypothesis of reward: past and current status. Trends Neurosci, 22:521-7.

- Spanagel R, Hölter SM. (2000). Pharmacological validation of a new animal model of alcoholism. $J$ Neural Transm, 107:669-80.

- Spealman RD, Bergman J, Madras BK, Melia KF. (1991). Discriminative stimulus effects of cocaine in squirrel monkeys: involvement of dopamine receptor subtypes. J Pharmacol Exp Ther, 258:945-53.

- Spealman RD, Bergman J. (1992). Modulation of the discriminative stimulus effects of cocaine by mu and kappa opioids. J Pharmacol Exp Ther, 261: 607615.

- Statistics on drug use in Australia 2006. (2007). Australian Institute of Health and Welfare, Canberra.

- Steketee J. (2005). Cortical mechanisms of cocaine sensitization. Crit Rev Neurobiol, 17:69-86.

- Stevenson GW, Wentland MP, Bidlack JM, Mello NK, Negus SS. (2004). Effects of the mixed-action kappa/mu opioid agonist 8-carboxamidocyclazocine on cocaine- and food-maintained responding in rhesus monkeys. Eur $J$ Pharmacol, 506:133-141. 
- Stewart J, de Wit H, Eikelboom R. (1984). Role of unconditioned and conditioned drug effects in the self-administration of opiates and stimulants. Psychol Rev, 91: 251-268.

- Stewart J. (2000). Pathways to relapse: the neurobiology of drug- and stressinduced relapse to drug-taking. J Psychiatry Neurosci, 25:125-136.

- Stimmel B, Kreek MJ. (2000). Neurobiology of addictive behaviors and its relationship to methadone maintenance. Mt Sinai J Med, 67:375-80.

- Stretch R, Gerber GJ, Wood SM. (1971). Factors affecting behaviour maintained by response-contingent intravenous infusions of amphetamine in squirrel monkeys. Can J Physiol Pharmacol, 49:581-589.

- Substance abuse and mental health services administration. (2009). www.samhsa.gov.

- Sughondhabirom A, Jain D, Gueorguieva R, Coric V, Berman R, Lynch WJ, Self D, Jatlow P, Malison RT. (2005). A paradigm to investigate the selfregulation of cocaine administration in humans. Psychopharmacology, 180:43646.

- Sun W, Xue Y, Huang Z, Steketee J. (2010). Regulation of cocaine-reinstated drug-seeking behavior by $\kappa$-opioid receptors in the ventral tegmental area of rats. Psychopharmacology, 210: 179-188.

- Suranyi-Cadotte BE, Bodnoff SR, Welner SA. (1990). Antidepressant-anxiolytic interactions: involvement of the benzodiazepine-GABA and serotonin systems. Prog Neuropsychopharmacol Biol Psychiatry, 14:633-54.

- Svingos A, Chavkin C, Colago EE, Pickel VM. (2001). Major coexpression of kappa-opioid receptors and the dopamine transporter in nucleus accumbens axonal profiles. Synapse, 42:185-192. 
- Szumlinski KK, Maisonneuve IM, Glick SD. (2000). Differential effects of ibogaine on behavioural and dopamine sensitization to cocaine. Eur $J$ Pharmacol, 398: 259-262.

- Tachibana S, Araki K, Ohya S, Yoshida S. (1982). Isolation and structure of dynorphin, an opioid peptide, from porcine duodenum. Nature, 295:339-340

- Tacker DH, Okorodudu AO. (2004). Evidence for injurious effect of cocaethylene in human microvascular endothelial cells. Clin Chim Acta, 345:6977.

- Tao R, Auerbach SB. (2005). Mu-Opioids disinhibit and Kappa-opioids inhibit serotonin efflux in the dorsal raphe nucleus. Brain Res, 1049: 70-79.

- Taylor WA, Gold MS. (1990). Pharmacologic approaches to the treatment of cocaine dependence. West J Med., 152: 573-577.

- The state of drug problem in Europe, Annual report 2009. (2009). European monitoring centre for drugs and drug addiction.

- Thompson AC, Zapata A, Justice JB Jr, Vaughan RA, Sharpe LG, Shippenberg TS (2000). Kappa-opioid receptor activation modifies dopamine uptake in the nucleus accumbens and opposes the effects of cocaine. J Neurosci 20:9333-9340.

- Thorndike EL. (1911). Animal intelligence: Experimental studies. New York: Macmillan

- Tidgewell K, Harding WW, Lozama A, Cobb H, Shah K, Kannan P, et al. (2006). Synthesis of Salvinorin A Analogues as Opioid Receptor Probes. J Nat Prod, 69: 914-918.

- Tidgewell K, Groer CE, Harding WW, Lozama A, Schmidt M, Marquam A, et al. (2008). Herkinorin Analogues with Differential Beta-Arrestin-2 Interactions. J Med Chem, 51: 2421-2431. 
- Tiihonen J, Kuoppasalmi K, Föhr J, Tuomola P, Kuikanmäki O, Vorma H, Sokero P, Haukka J, Meririnne E. (2007). A comparison of aripiprazole, methylphenidate, and placebo for amphetamine dependence. Am J Psychiatry, $164: 160-2$

- Todtenkopf M, Carreiras T, Melloni R, Stellar J. (2002). The dorsomedial shell of the nucleus accumbens facilitates cocaine-induced locomotor activity during the induction of behavioral sensitization. Behav Brain Res, 131: 9-16.

- Todtenkopf M, Marcus J, Portoghese P, Carlezon WA Jr (2004). Effects of kappa-opioid receptor ligands on intracranial self-stimulation in rats. Psychopharmacology 172:463-470.

- Tomasiewicz HC, Todtenkopf MS, Chartoff EH, Cohen BM, Carlezon WA Jr (2008). The kappa-opioid agonist U69593 blocks cocaine-induced enhancement of brain stimulation reward. Biol Psychiatry 64:982-988.

- Torres GE, Gainetdinov RR, Caron MG. (2003). Plasma membrane monoamine transporters: structure, regulation and function. Nat Rev Neurosci, 4:13-25.

- Tran-Nguyen T, Fuchs R, Coffey G, O’Dell L, Baker D, Neisewander J. (1998). Time-dependent changes in cocaineseeking behavior and dopamine overflow in the amygdala during cocaine withdrawal. Neuropsychopharmacology 19, 48-59.

- Ushijima, M.A. Carino, A. Horita (1995). Involvement of $\mathrm{D}_{1}$ and $\mathrm{D}_{2}$ dopamine systems in the behavioral effects of cocaine in rats. Pharmacol Biochem Behav 52: 737-741.

- Velázquez-Sánchez C, Ferragud A, Hernández-Rabaza V, Nácher A, Merino V, Cardá M, Murga J, Canales JJ. (2009). The dopamine uptake inhibitor 3 alpha[bis(4'-fluorophenyl)metoxy]-tropane reduces cocaine-induced early-gene expression, locomotor activity, and conditioned reward. Neuropsychopharmacology, 34:2497-507. 
- Velázquez-Sánchez C, Ferragud A, Murga J, Cardá M, Canales JJ.(2010). The high affinity dopamine uptake inhibitor, JHW 007, blocks cocaine-induced reward, locomotor stimulation and sensitization. Eur Neuropsychopharmacol, 20:501-8.

- Valdés LJ 3rd, Díaz JL, Paul AG (1983). Ethnopharmacology of ska María Pastora (Salvia divinorum, Epling and Játiva-M.). J Ethnopharmacol 7:287-312.

- Valdés LJ 3rd (1994). Salvia divinorum and the unique diterpene hallucinogen, Salvinorin (divinorin) A. J Psychoactive Drugs 26:277-83.

- Valentino RJ, Lucki I, Van Bockstaele E. (2010). Corticotropin-releasing factor in the dorsal raphe nucleus: Linking stress coping and addiction. Brain Res, 1314: 29-37.

- Van Bockstaele EJ, Pickel VM. (1995) GABA-containing neurons in the ventral tegmental area project to the nucleus accumbens in rat brain. Brain Res, 682:215-221.

- Van Bockstaele EJ, Reyes BAS, Valentino RJ. (2010). The locus coeruleus: A key nucleus where stress and opioids intersect to mediate vulnerability to opiate abuse. Brain Res, 1314: 162-174.

- Vanderschuren LJ, Kalivas PW. (2000). Alterations in dopaminergic and glutamatergic transmission in the induction and expression of behavioral sensitization: a critical review of preclinical studies. Psychopharmacology, 151:99-120.

- Vanderschuren LJ, Schoffelmeer AN, Wardeh G, De Vries TJ (2000). Dissociable effects of the kappa-opioid receptor agonists bremazocine, U69593, and $\mathrm{U} 50488 \mathrm{H}$ on locomotor activity and long-term behavioral sensitization induced by amphetamine and cocaine. Psychopharmacology 150:35-44. 
- Velayudhan A, Sunitha TA, Balachander S, Reddy JY, Khanna S. (1999). A study of platelet serotonin receptor in mania. Biol Psychiatry, 45:1059-62.

- Verdejo-García A, Pérez-García M, Sánchez-Barrera M, Rodriguez-Fernández A, Gómez-Río M. (2007). Neuroimaging and drug addiction: neuroanatomical correlates of cocaine, opiates, cannabis and ecstasy abuse. Rev Neurol., 44(7), $432-439$

- Vogt M. (1969). Release from brain tissue of compounds with possible transmitter function: interaction of drugs with these substances. Br J Pharmacol, $37: 325-37$.

- Vonvoigtlander P, Lewis R. (1982). U-50,488, a selective kappa opioid agonist: Comparison to other reputed kappa agonists. Prog Neuropsychopharmacol Biol Psychiatry, 6: 467-470.

- Vonvoigtlander PF, Lahti RA, Ludens JH. (1983). U-50,488: a selective and structurally novel non-Mu (kappa) opioid agonist. J Pharmacol Exp Ther, 224: $7-12$.

- Vortherms TA, Roth BL. (2006). Salvinorin A: from natural product to human therapeutics. Mol. Interventions, 6:257-265.

- Vorspan F, Bellais L, Keijzer L, Lépine JP. (2008). An open-label study of aripiprazole in nonschizophrenic crack-dependent patients. $J$ Clin Psychopharmacol, 28:570-2.

- Wadenberg M. (2003). A review of the properties of spiradoline: a potent and selective kappa-opioid receptor agonist. CNS Drug Rev, 9: 187-198.

- Walsh S, Strain E, Abreu M, Bigelow G (2001). Enadoline, a selective kappa opioid agonist: comparison with butorphanol and hydromorphone in humans. Psychopharmacology 157:151-162. 
- Walsh SL, Geter-Douglas B, Strain EC, Bigelow GE. (2001). Enadoline and Butorphanol: Evaluation of Kappa-Agonists on Cocaine Pharmacodynamics and Cocaine Self-Administration in Humans. J Pharmacol Exp Ther, 299: 147-158.

- Wang DS, Sternbach G, Varon J. (1998). Nalmefene: A Long-Acting Opioid Antagonist. Clinical Applications In Emergency Medicine. J Emerg Med, 16: 471-475.

- Wang D, Raehal KM, Lin ET, Lowery JJ, Kieffer BL, Bilsky EJ, Sadée W. (2004). Basal signaling activity of mu opioid receptor in mouse brain: role in narcotic dependence. J Pharmacol Exp Ther, 308:512-20.

- Wang Y, Tang K, Inan S, Siebert D, Holzgrabe U, Lee Y, Huang P, Li JG, Cowan A, Liu-Chen LY. (2005). Comparison of pharmacological activities of three distinct kappa-ligands (salvinorin A, TRK-820 and 3FLB) on Kappaopioid receptors in-vitro and their antipruritic and antinociceptive activities invivo. J Pharmacol Exp Ther, 312:220-230.

- Wang Y, Chen Y, Xu W, Lee DYW, Ma Z, Rawls SM, et al. (2008). 2Methoxymethyl-Salvinorin B Is a Potent Kappa-Opioid Receptor Agonist with Longer Lasting Action in Vivo Than Salvinorin A. J Pharmacol Exp Ther, 324: 1073-1083.

- Wang YJ, Tao YM, Li FY, Wang YH, Xu XJ, Chen J, Cao YL, Chi ZQ, Neumeyer JL, Zhang A, Liu JG. (2009). Pharmacological characterization of ATPM[(-)-3-aminothiazolo[5,4-b]-N-cyclopropylmethylmorphinan hydrochloride], a novel mixed kappa-agonist and mu-agonist/-antagonist that attenuates morphine antinociceptive tolerance and heroin self-administration behavior. J Pharmacol Exp Ther, 329:306-13.

- Watts VJ, Neve KA. (2005). Sensitization of adenylate cyclase by G[alpha]i/ocoupled receptors. Pharmacol Ther, 106: 405-421. 
- Wee S, Koob, G. (2010). The role of the dynorphin- $\kappa$ opioid system in the reinforcing effects of drugs of abuse. Psychopharmacology, 210: 121-135.

- Weiss F, Markou A, Lorang MT, Koob GF. (1992). Basal extracellular dopamine levels in the nucleus accumbens are decreased during cocaine withdrawal after unlimited-access self-administration. Brain Res, 593:314-318.

- Weissenborn R, Yackey M, Koob GF, Weiss F. (1995). Measures of cocaineseeking behavior using a multiple schedule of food and drug self-administration in rats. Drug Alcohol Depend, 38: 237-246.

- Wexler BE, Gottschalk CH, Fulbright RK, Prohovnik I, Lacadie CM, Rounsaville BJ, Gore JC. (2001). Functional magnetic resonance imaging of cocaine craving, Am. J. Psychiatry, 158: 86-95.

- White FJ. (1990). Electrophysiological basis of the reinforcing effects of cocaine. Behav Pharmacol, 1:303-315.

- Willmore-Fordham CB, Krall DM, McCurdy CR, Kinder DH (2007). The hallucinogen derived from Salvia divinorum, salvinorin A, has kappa-opioid agonist discriminative stimulus effects in rats. Neuropharmacology 53:481-486.

- Wise RA, Bozarth MA. (1984). Brain reward circuitry: Four circuit elements "wired" in apparent series. Brain Res Bull, 297: 265-273.

- Wise RA, Rompre PP. (1989). Brain dopamine and reward. Ann Rev Psychol, 40:191-225.

- Wise RA, Schwartz HV. (1981). Pimozide attenuates acquisition of leverpressing for food in rats. Pharmacol Biochem Behav, 15: 655-656.

- Wolf ME. (2010). The Bermuda Triangle of cocaine-induced neuroadaptations. Trends Neurosci, 33:391-8. 
- Woolverton WL. (1986). Effects of a D1 and a D2 dopamine antagonist on the self-administration of cocaine and piribedil by rhesus monkeys. Pharmacol Biochem Behav, 24:531-5.

- World Drug Report. (2007). United Nations Office on Drugs and Crime.

- Worley CM, Valadez A, Schenk S. (1994). Reinstatement of extinguished cocaine-taking behavior by cocaine and caffeine. Pharmacol Biochem Behav, 48: 217-221.

- Wightman DS, Foster VJ, Krishen A, Richard NE, Modell JG. (2010). Metaanalysis of suicidality in placebo-controlled clinical trials of adults taking bupropion. Prim Care Companion J Clin Psychiatry. 12(5): pii: PCC.09m00894.

- Xu H, Arita H, Hayashida M, Zhang L, Sekiyama H, Hanaoka K. (2006). Painrelieving effects of processed Aconiti tuber in CCI-neuropathic rats. $J$ Ethnopharmacol, 103: 392-397.

- Xu YT, Kaushal N, Shaikh J, Wilson LL, Mésangeau C, McCurdy CR, Matsumoto RR (2010). A novel substituted piperazine, CM156, attenuates the stimulant and toxic effects of cocaine in mice. J Pharmacol Exp Ther 333:491500.

- Yan F, Roth BL (2004). Salvinorin A: A novel and highly selective kappaopioid receptor agonist. Life Sci 75:2615-2619.

- Yilmaz B, Sandal S, Canpolat S, Kutlu, Kelestimur H. (2006). Kappa Opioid Modulation of Serotonergic Neurotransmission in the Hypothalamus, Hippocampus and Striatum in the Male Rat. Fırat Tip Dergisi, 11: 12-15.

- Zahniser NR, Larson GA, Gerhardt GA.(1999). In vivo dopamine clearance rate in rat striatum: regulation by extracellular dopamine concentration and dopamine transporter inhibitors. J Pharmacol Exp Ther, 289:266-77. 
- Zahniser NR, Doolen S. (2001). Chronic and acute regulation of $\mathrm{Na}+/ \mathrm{Cl}-$ dependent neurotransmitter transporters: drugs, substrates, presynaptic receptors, and signaling systems. Pharmacol Ther, 92: 21-55.

- Zakharova E, Collins SL, Åberg M, Kumar A, Fernandez JB, Izenwasser, S. (2008). Depletion of serotonin decreases the effects of the kappa-opioid receptor agonist U-69593 on cocaine-stimulated activity. Eur J Pharmacol, 586:123-129.

- Zapata A, Kivell B, Han Y, Javitch JA, Bolan EA, Kuraguntla D, et al. (2007). Regulation of Dopamine Transporter Function and Cell Surface Expression by D3 Dopamine Receptors. J Biol Chem, 282: 35842-35854.

- Zhang Y, Butelman E, Schlussman S, Ho A, Kreek M. (2004). Effect of the kappa-opioid agonist R-84760 on cocaine-induced increases in striatal dopamine levels and cocaine-induced place preference in $\mathrm{C} 57 \mathrm{BL} / 6 \mathrm{~J}$ mice. Psychopharmacology, 173:146-152.

- Zhang Y, Butelman ER, Schlussman SD, Ho A, Kreek MJ (2005). Effects of the plant-derived hallucinogen salvinorin A on basal dopamine levels in the caudate putamen and in a conditioned place aversion assay in mice: agonist actions at kappa opioid receptors. Psychopharmacology 179:551-558. 


\section{$\underline{\text { Appendix }}$}

\section{Appendix for Chapter 2}

\section{Test for patency of catheters (Pentobarbital test)}

- Initially try and withdraw some blood from the catheter using a syringe which has penicillin in heparinised saline.

- If you observe blood in the syringe, the line is OK. Flush back blood with 0.1 $\mathrm{mL}$ of heparinised penicillin solution.

- If you don't observe blood, then infuse $0.1 \mathrm{~mL}$ of pentobarbital (Provet, Auckland; $5 \mathrm{mg} / \mathrm{mL}$ ). If the animal becomes unconscious, then the line is still OK. If the animal does not get unconscious, then the rat has either a chest leak or head leak and needs unblocking of its line.

- The cather patency test was performed when rats were not being trained for self-administration sessions.

\section{Appendix for Chapter 4}

\section{Recipe for $\mathrm{LB}$ Broth (for $500 \mathrm{~mL}$ )}

- $\quad$ Tryptone (OXOID, UK) $5 \mathrm{~g}$

- Sodium Chloride (LabServ) $5 \mathrm{~g}$

- Yeast Extract (Sigma) 2.5 g

- Add distilled water and autoclave it.

2. Recipe for Agar plates (200 mL; each plate takes approx $20 \mathrm{~mL}$ )

- Agar (Fischer Scientific)2 g

- Tryptone $2 \mathrm{~g}$

- Sodium Chloride $2 \mathrm{~g}$

- Yeast Extract $1 \mathrm{~g}$ 
- Add distilled water and autoclave it. After autoclaving, cool the flask (until the flask is slightly warm) then add appropriate antibiotic, ie Ampicillin (Sigma, MO) for KOPr plasmid preparation $(100 \mu \mathrm{g} / \mathrm{mL})$ and Kanamycin (Tocris, UK) for SERT plasmid $(50 \mu \mathrm{g} / \mathrm{mL})$.

- Slightly shake the flask for gently mixing and then pour in the agar plates. Allow the agar to set for few hours and then store it in refrigerator. These plates can be stored up to 2 months.

\section{Method for GFP-hSERT and myc-rKOPr plasmid Preparation:}

- Day 1:- Take a scraping of the frozen transformed bacteria transfected with cDNA for GFP-hSERT (generous gift from Dr. Samanda Ramamoorthy) and myc-rKOPR (generous gift from Dr. Lakshmi Devi) from the vial stored at $80{ }^{\circ} \mathrm{C}$. Plate the bacteria on a fresh agar plate. Incubate the plate for $24 \mathrm{hr}$ at $37{ }^{\circ} \mathrm{C}$ and allow the bacterial colony to grow on agar plate in humid condition (place some water in a conical flask in the incubator).

- Day 2:- Add $2 \mathrm{~mL}$ of sterile $\mathrm{LB}$ broth in a sterile $15 \mathrm{~mL}$ tube which contains $100 \mu \mathrm{g} / \mathrm{mL}$ of ampicillin (for myc-rKOPr) and $50 \mu \mathrm{g} / \mathrm{mL}$ of kanamycin (for GFP-hSERT). Select a middle sized colony of bacteria from the agar plate and transfer it using a sterile pipette into the $15 \mathrm{~mL}$ tube and incubate for $24 \mathrm{hr}$ at $37^{\circ} \mathrm{C}$ with constant shaking at 280 rotation per minute in humid condition.

- Day 3:- Add $200 \mu \mathrm{L}$ of the bacterial culture to $200 \mathrm{~mL}$ of $\mathrm{LB}$ broth which contains appropriate antibiotic in proper concentrations and incubate for $24 \mathrm{hr}$ at $37{ }^{\circ} \mathrm{C}$ with constant shaking at 280 rotation per minute in humid condition.

- Day 4:- Centrifuge samples for $15-25$ min at $4{ }^{\circ} \mathrm{C}$. (Dupont Centrifuge, RC5C; GSA 1 rotor; rotor code: 10; Sorvall Instruments). Dispose off the supernatant and keep the pellet on ice. Then follow the instructions mentioned in the manufacturer's catalogue (Quagen) 
4. Media for HEK-293 cells. (for $100 \mathrm{~mL}$ )

- Dulbecco Modified Eagle's Medium (Gibco, Invitrogen) 89 mL.

- Fetal Calf Serum $10 \mathrm{~mL}$ (Invitrogen)

- Penstrap (Invitrogen) $1 \mathrm{~mL}$.

5. Media for HEK-293 cells. (without penstrap; for $100 \mathrm{~mL}$ )

- Dulbecco Modified Eagle's Medium 90 mL.

- Fetal Calf Serum $10 \mathrm{~mL}$.

6. Transient transfection of HEK-293 cells with myc-rKOPr and GFP-SERT.

- Mix A:- $3 \mu \mathrm{L}$ Lipofectamine (Invitrogen) $+47 \mu \mathrm{L}$ OptiMEM (Invitrogen) (Keep it for $5 \mathrm{~min}$ ).

- Mix B:- $(0.4 \mu \mathrm{g} / \mathrm{mL}$ GFP-hSERT + $0.6 \mu \mathrm{g} / \mathrm{mL}$ myc-rKOPr $)+$ make volume upto $50 \mu \mathrm{L}$ using OptiMEM.

- $\operatorname{Mix} \mathrm{A}+\operatorname{Mix} \mathrm{B}$ (Keep this for $20 \mathrm{~min})$

- Add $100 \mu \mathrm{L}$ of Mix A + Mix B to each culture dish (1 mL/dish) and return dish back to incubator.

\section{Krebs Ringer Herpes buffer (KRH).}

- Sodium Chloride $130 \mathrm{mM}$

- Potassium Chloride (LabServ) $1.3 \mathrm{mM}$

- Calcium Chloride (Fischer Scientific) $2.2 \mathrm{mM}$

- Magnesium sulphate (LabServ) $1.2 \mathrm{mM}$

- Potassium Dihydrogen Phosphate (LabServ) $1.2 \mathrm{mM}$

- $\operatorname{HEPES}$ (Sigma) $10 \mathrm{mM}$

- D-Glucose (Sigma) 10mM 


\title{
Effect of kappa-opioid receptor agonists U69593, U50488H, spiradoline and salvinorin A on cocaine-induced drug-seeking in rats
}

\author{
Aashish S. Morani ${ }^{a}$, Bronwyn Kivell ${ }^{\text {a }}$, Thomas E. Prisinzano ${ }^{\mathrm{b}}$, Susan Schenk ${ }^{\mathrm{c}, *}$ \\ a School of Biological Sciences, Victoria University of Wellington, P.O. Box 600, Wellington, New Zealand \\ ${ }^{\mathrm{b}}$ Department of Medicinal Chemistry, University of Kansas, Lawrence, Kansas 6604, USA \\ ' School of Psychology, Victoria University of Wellington, P.O. Box 600, Wellington, New Zealand
}

\section{A R T I C L E I N F O}

\section{Article history:}

Received 29 April 2009

Received in revised form 29 July 2009

Accepted 1 September 2009

Available online 10 September 2009

\section{Keywords:}

Cocaine self-administration

Kappa-opioid agonist

U69593

$\mathrm{U} 50488 \mathrm{H}$

Spiradoline

Salvinorin A

Drug-seeking

\begin{abstract}
A B S T R A C T
Our previous work indicated that pretreatment with the selective kappa-opioid receptor (KOPr) agonist, U69593, attenuated the ability of priming injections of cocaine to reinstate extinguished cocaine-seeking behavior. The present study expanded these initial tests to include other traditional KOPr agonists, U50488H, spiradoline (SPR), and salvinorin A (Sal A), an active constituent of the plant Salvia divinorum. Following acquisition and stabilization of cocaine self-administration, cocaine-produced drug-seeking was measured. This test was conducted in a single day and comprised an initial phase of self-administration, followed by a phase of extinguished responding. The final phase examined reinstatement of extinguished cocaine selfadministration followed by a priming injection of cocaine $(20.0 \mathrm{mg} / \mathrm{kg}$, intraperitoneal (I.P.)) in combination with the various KOPr agonists. Cocaine-induced drug-seeking was attenuated by pretreatment with U69593 $(0.3 \mathrm{mg} / \mathrm{kg}$, subcutaneous (S.C.)), U50488H (30.0 mg/kg, I.P.), SPR (1.0, $3.0 \mathrm{mg} / \mathrm{kg}$, I.P.) and Sal A (0.3, $1.0 \mathrm{mg} /$ $\mathrm{kg}$, I.P.). Sal A $(0.3,1.0 \mathrm{mg} / \mathrm{kg}$, I.P.) had no effect on operant responding to obtain sucrose reinforcement or on cocaine-induced hyperactivity. These findings show that Sal A, like other traditional KOPr agonists attenuates cocaine-induced drug-seeking behavior.
\end{abstract}

(c) 2009 Elsevier Inc. All rights reserved.

\section{Introduction}

Prior studies have demonstrated that KOPr agonists attenuate some of the neuro-chemical and behavioral effects of drugs of abuse (Heidbreder et al., 1993, 1995; Shippenberg et al., 1996; Thompson et al., 2000). These effects might be due to the interactions between KOPr and dopaminergic systems since pretreatment with kappa-opioid agonists decreased dopamine (DA) concentrations in terminal regions (Devine et al., 1993; Shippenberg et al., 1994; Maisonneuve et al., 1994; Heidbreder and Shippenberg, 1994; Heidbreder et al., 1996) and attenuated cocaine- (Shippenberg et al., 1994), amphetamine- (Gray et al., 1999) and heroin- (Xi et al., 1998) induced DA release in the ventral striatum. Also, many behavioral effects of psycho-stimulants like cocaine (Heidbreder et al., 1993, 1995), amphetamine (Gray et al., 1999) and nicotine (Hahn et al., 2000) were decreased by prior administration of KOPr agonists. Additionally, pretreatment with KOPr activating compounds attenuated cocaine (Glick et al., 1995; Mello and Negus, 1996, 1998; Negus et al., 1997; Schenk et al., 1999; Schenk and Partridge, 2001), morphine (Glick et al., 1995) and heroin (Xi et al., 1998) selfadministration in laboratory animals. These findings suggest the

\footnotetext{
* Corresponding author. Tel.: +64 4463 6034; fax: +64 44635402.

E-mail addresses: aashish.morani@vuw.ac.nz (A.S. Morani),

Bronwyn.kivell@vuw.ac.nz (B. Kivell), prisinza@ku.edu (T.E. Prisinzano), Susan.schenk@vuw.ac.nz (S. Schenk).
}

possible role of KOPr agonists as pharmacotherapeutics in the treatment of addiction (Mello and Negus, 1998; Prisinzano et al., 2005; Shippenberg et al., 2007; Willmore-Fordham et al., 2007; Prisinzano and Rothman, 2008; Tomasiewicz et al., 2008).

During a binge of cocaine self-administration in abusers there is tolerance to many of the subjective effects (Fischman et al., 1985). Self-administration and drug craving, however, continue relatively unabated. Since exposure to cocaine produces craving for more cocaine (Jaffe et al., 1989), it is possible that continued exposure to cocaine during a binge maintains a cycle of drug-seeking and drugtaking, even after tolerance develops. If so, identification of factors that reduce the ability of cocaine to produce drug-seeking would be an important step in the development of effective treatments for cocaine abuse.

Animal models of drug-seeking have contributed greatly to investigations of these factors. A number of laboratories have used procedures developed by de Wit and Stewart (1981) to demonstrate the ability of cocaine to reinstate extinguished cocaine self-administration behavior. This effect appears to be mediated, at least in part, by dopaminergic mechanisms since cocaine-produced drug-seeking was attenuated by pretreatment with dopaminergic agonists and antagonists (Self et al., 1996; Khroyan et al., 2000, 2003; Alleweireldt et al., 2002).

Pretreatment with the kappa-opioid receptor agonist, U69593 attenuated cocaine-produced reinstatement of extinguished cocaine- 
taking behavior (Schenk et al., 1999, 2000). This effect was somewhat specific since reinstatement produced by experimenter-administered injections of the dopamine uptake inhibitors, GBR 12909 or WIN 35428, was not attenuated by pretreatment with U69593 (Schenk et al., 2000). The attenuation of drug-seeking involved effects at central KOPrs, as prior intracerebroventricular administration of nor-BNI, a kappa-opioid antagonist, reversed the effects of U69593 (Schenk et al., 1999).

Sal A, a neoclerodane diterpene, is an active constituent of the hallucinogenic sage, Salvia divinorum, and has been used in traditional, spiritual and ethnopharmacological practices by the Mazatec Indians of Oaxaca, Mexico (Valdes et al., 1983; Valdes, 1994; Siebert, 1994). Sal A binds selectively to the KOPr (Roth et al., 2002; Yan and Roth, 2004; Prisinzano, 2005) with greater efficacy than U69593 and U50488H (Chavkin et al., 2004). It has a rapid onset of action and a short elimination half life (56.6 $\pm 24.8 \mathrm{~min})$ (Schmidt et al., 2005; Butelman et al., 2009). Accordingly, there has been recent interest in understanding the neuropharmacology of Sal A and comparing it with traditional KOPr agonists.

Sal A dose dependently produced antinociception in tail flick, hot plate and acetic acid induced writhing in mice that was antagonized by nor-BNI (McCurdy et al., 2006; John et al., 2006). Sal A also produced discriminative stimulus effect in rats (Willmore-Fordham et al., 2007; Baker et al., 2009) and rhesus monkeys (Butelman et al., 2004), decreased mobility in the forced swim test (Carlezon et al., 2006) and produced motor inco-ordination in mice (Fantegrossi et al., 2005; Zhang et al., 2005). The aim of our current study was to determine whether Sal A has similar effects to other KOPr agonists, U69593, U50488H and SPR on cocaine-produced drug-seeking in rats.

\section{Methods}

\subsection{Subjects}

Male Sprague-Dawley rats weighing 325-350 g were used. They were housed individually in hanging polycarbonate cages. The humidity (55\%) and temperature $\left(19-21{ }^{\circ} \mathrm{C}\right)$ were controlled and food and water were freely available except during testing. Animals tested for sucrose self-administration had free access to water at all times within the home cage and were maintained at approximately $85 \%$ of their initial feeding weight during the experiments by restricting access to food. The animal colony was maintained in the animal facility at School of Psychology, Victoria University of Wellington. All the experiments were conducted in accordance with the guidelines of the Animal Ethics Committee of Victoria University of Wellington. Lights were maintained on a 12:12 h cycle with lights on at 0700 .

\subsection{Surgery}

Under deep anesthesia produced by ketamine/xylacine $(90 / 9 \mathrm{mg} / \mathrm{kg}$, I.P.), a silastic catheter was placed in the right jugular vein. The external jugular vein was isolated, the catheter was inserted and the distal end (22 gauge stainless steel tubing) was passed subcutaneously to an exposed portion of the skull where it was fixed to embedded jeweler's screws with dental acrylic.

Each day, the catheters were infused with $0.1 \mathrm{ml}$ of a sterile saline solution containing heparin $(30.0 \mathrm{U} / \mathrm{ml})$, penicillin $\mathrm{G}$ Potassium $(250,000 \mathrm{U} / \mathrm{ml})$ and streptokinase $(8000 \mathrm{U} / \mathrm{ml})$ to prevent infection and the formation of clots and fibroids. The rats were allowed five days post surgery for recovery.

\subsection{Apparatus}

\subsubsection{Cocaine self-administration}

Self-administration testing was carried out in a humidity (55\%) and temperature $\left(19-21^{\circ} \mathrm{C}\right)$ controlled environment in standard operant chambers (Med Associates, ENV-001) equipped with 2 levers. Depres- sion of one lever (the active lever) resulted in a $0.1 \mathrm{ml}$ intravenous infusion of cocaine $\mathrm{HCl}$ dissolved in sterile physiological saline containing heparin $(3.0 \mathrm{U} / \mathrm{ml})$. Infusions were of $12 \mathrm{~s}$ duration. Coincident with drug delivery was the illumination of a stimulus light located directly above the active lever. This stimulus light remained illuminated throughout each $12 \mathrm{~s}$ infusion. Depression of the other lever (the inactive lever) was without programmed consequence.

Rats were maintained in the animal colony except during testing. Immediately prior to each daily test, the catheter lines were infused with $0.1 \mathrm{ml}$ of the heparin-penicillin-streptokinase solution. The stainless steel catheter was connected to a length of microbore tubing and connected to the syringe. At the end of each test, the lines were again infused with $0.1 \mathrm{ml}$ of the heparin-penicillin-streptokinase solution, the stainless steel tubing was plugged and the animal was returned to its home cage. Drug delivery and data acquisition were controlled by Med Associates software. Cocaine deliveries were made via mechanical pumps (Razel, Model A with $1.0 \mathrm{rpm}$ motor equipped with $20 \mathrm{ml}$ syringe).

\subsubsection{Sucrose self-administration}

Training and testing procedures were conducted in eight standard operant chambers (Med Associates, ENV001) in an unlit, sound attenuating room. Operant chambers were equipped with two retractable levers and a sucrose bottle delivering $0.1 \mathrm{ml}$ of $10 \%$ sucrose solution onto a tray on the chamber wall according to the imposed schedule of reinforcement. Sucrose delivery and data acquisition were controlled by Med Associates software. Experiments were conducted between 0900 and 1600 hours.

\subsubsection{Locomotor activity tests}

Eight open field chambers (Med Associates) equipped with two banks of sixteen photocells on each wall were used to measure horizontal locomotion. The open field boxes were interfaced with a microcomputer located in an adjacent laboratory. Testing was conducted in the dark between 1000 and 1600 hours. White noise was continually present to mask extraneous disturbances.

\subsection{Procedure}

\subsubsection{Cocaine self-administration training}

Acquisition of cocaine self-administration was monitored during daily 2 h sessions. Each session began with an experimenter-delivered infusion of cocaine $(0.5 \mathrm{mg} / \mathrm{kg} /$ infusion $)$. Thereafter, depression of the active lever produced automated cocaine infusions according to an FR-1 schedule of reinforcement. The criterion for acquisition of cocaine selfadministration consisted of at least 20 reinforced responses $(10 \mathrm{mg} / \mathrm{kg}$ ) during the $2 \mathrm{~h}$ session and a ratio of active:inactive lever responses of at least 2:1. Self-administration was considered acquired when these criteria were met for three consecutive days. Following acquisition, the response requirements were increased to FR-5. Daily 2 h sessions were conducted until there was less than $20 \%$ variation in responding on three consecutive days. During training, the cocaine infusion was always paired with the illumination of a house light located directly above the active lever.

\subsubsection{Cocaine reinstatement test}

Once responding on the FR-5 schedule was stable, the effect of prior administration of the kappa-opioid agonists, U69593, U50488H, SPR and Sal A on drug-seeking produced by cocaine was measured. As with our previous studies (Schenk et al., 1999, 2000), this test was conducted in a single day and consisted of three phases. The first phase was comprised of a $1 \mathrm{~h}$ period of cocaine self-administration $(0.5 \mathrm{mg} / \mathrm{kg} /$ infusion, FR-5 schedule of reinforcement) in which the light stimulus was paired with cocaine infusions. After the $1 \mathrm{~h}$ self-administration period, the cocaine solution was replaced with heperanized saline and responding was reinforced with this vehicle infusion (FR-5 schedule of 
reinforcement) and illumination of the light stimulus. Responding during this phase was measured for $3 \mathrm{~h}$. At the start of the third phase during which responding was again reinforced according to an FR-5 schedule of reinforcement with an infusion of vehicle solution and illumination of the light stimulus, separate groups of rats $(n=5-7$ per group) received an injection of U69593 (0.03, $0.1 \mathrm{or} 0.3 \mathrm{mg} / \mathrm{kg}$, S.C.), U50488H (3.0, 10.0 or $30.0 \mathrm{mg} / \mathrm{kg}$, I.P.), SPR (0.3, 1.0 or $3.0 \mathrm{mg} / \mathrm{kg}$, I.P.), Sal A (0.1, 0.3 and $1 \mathrm{mg} / \mathrm{kg}$, I.P.) or vehicle. These injections were administered either 5 (Sal A), 15 (U69593) or 30 (U50488H, SPR) min prior to an injection of cocaine $\mathrm{HCl}(20 \mathrm{mg} / \mathrm{kg}$, I.P.). Responding was measured for $60 \mathrm{~min}$ following the cocaine injection.

\subsubsection{Sucrose reinforcement training and test}

Animals were trained to self-administer sucrose using an autoshaping procedure. Training sessions were 45 min duration on each of 10 training days. Once stable responding was produced, the animals were maintained on an FR1 schedule of reinforcement during which depression of the active lever (left lever) delivered $0.1 \mathrm{ml}$ of a $10 \%$ sucrose solution. Following acquisition, the response requirements were increased to FR-5. Daily $1 \mathrm{~h}$ sessions were conducted until there was less than $20 \%$ variation in responding for three consecutive days. Once responding on the FR-5 schedule was stable, the effect of Sal A on sucrose-reinforced responding was measured. Rats received an injection of either Sal A ( 0.3 or $1.0 \mathrm{mg} / \mathrm{kg}$, I.P.) or vehicle $5 \mathrm{~min}$ prior to sucrose self-administration testing and the number of responses was measured for $60 \mathrm{~min}$.

\subsubsection{Procedure for cocaine-produced locomotor activity}

On the test day, separate groups of rats ( $n=6$ per group) received an injection of Sal A ( 0.0 or $0.3 \mathrm{mg} / \mathrm{kg}$, I.P.) $5 \mathrm{~min}$ prior to an injection of cocaine $(20 \mathrm{mg} / \mathrm{kg}$, I.P.). Immediately following the second injection, the rats were placed in the activity chambers and total activity, a compilation of horizontal and vertical activity, was measured for a period of $60 \mathrm{~min}$.

\subsection{Data analysis}

The number of responses produced during the $1 \mathrm{~h}$ period following the cocaine injection $(20 \mathrm{mg} / \mathrm{kg}$, I.P.) at the start of phase 3 was measured. Sucrose-reinforced responding was measured for a $1 \mathrm{~h}$ period following Sal A or vehicle exposure. Statistical analysis for cocaine-induced reinstatement and sucrose reinforcement consisted of one-way ANOVAs followed by Tukey post-hoc comparisons where appropriate. Student $t$ test was applied for locomotion test.

\subsection{Drugs}

Cocaine $\mathrm{HCl}$ (Merck Pharmaceuticals, Palmerston North, New Zealand), SPR and U50488H (Sigma-Aldrich, St. Louis, MO) were dissolved in physiological saline. U69593 (National Institute of Drug Abuse) was dissolved in an aqueous solution of $25 \%$ propylene glycol. Sal A (isolated by Dr. Thomas E. Prisinzano, University of Kansas, Lawrence, KS) was suspended in 75\% DMSO. Sucrose was dissolved in tap water. Intravenous infusions were in a volume of $100 \mu$ and S.C. or I.P. injections were in a volume of $1 \mathrm{ml} / \mathrm{kg}$. All drug weights refer to the salt.

\section{Results}

Fig. 1 shows the responding during phases 1 and 2 for a representative group of rats from the present study. During the $1 \mathrm{~h}$ period of cocaine selfadministration, responding was high. When saline was substituted for cocaine during phase 2 , responding decreased to less than 20 responses during hour 3.

Fig. 2 shows the number of saline-reinforced responses produced during the $1 \mathrm{~h}$ period following the injection of cocaine at the start of phase 3 for rats that received systemic administration of the $\mathrm{KOPr}$

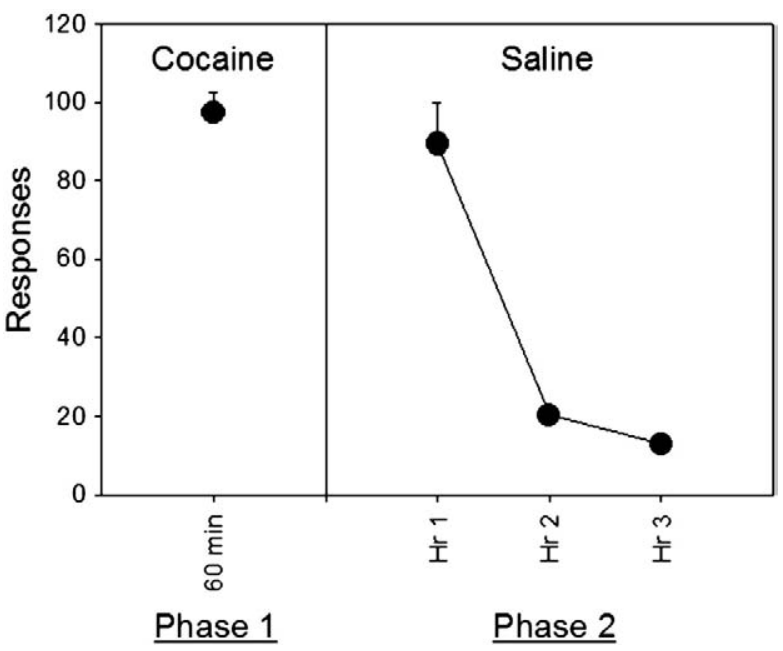

Fig. 1. Number of lever press responses (+SEM) during Phases 1 (cocaine self administration) and 2 (extinction) $(n=6)$. During phase 1 , responding is high $(97.0+$ 6.1 ) and by the end of phase 2 , after the cocaine solution had been replaced with saline, fewer than 20 responses per hour were produced $(16.3+1.3)$.

agonists, U69593, U50488H, SPR and SalA. Each of the KOPr agonists produced a dose-dependent reduction of cocaine-produced reinstatement (U50488H: $F(3,17)=4.277, p<0.05$; U69593: $F(3,20)=3.103$, $p<0.05)$; SPR: $(F(3,21)=7.899, p<0.01$; Sal A: $(F(3,23)=79.33$, $p<0.0001)$. Doses of $30.0 \mathrm{mg} / \mathrm{kg} \mathrm{U} 50488 \mathrm{H}, 0.3 \mathrm{mg} / \mathrm{kg} \mathrm{U69593,} 1.0$ or $3.0 \mathrm{mg} / \mathrm{kg}$ SPR and 0.3 or $1.0 \mathrm{mg} / \mathrm{kg}$ Sal A significantly decreased drugseeking $(p<0.05)$.

Fig. 3 shows the number of sucrose-reinforced responses produced during the $1 \mathrm{~h}$ period following the injection of Sal A $(0.3$ and $1.0 \mathrm{mg} / \mathrm{kg}$, I.P.). Sal A did not significantly decrease sucrose self-administration ( $F$ $(2,27)=0.06, \mathrm{NS})$.

Fig. 4 shows the effect of Sal A ( 0.0 or $0.3 \mathrm{mg} / \mathrm{kg}$, I.P.) on cocaineproduced locomotor activity. Sal A failed to decrease cocaineproduced hyperactivity.

\section{Discussion}

As has previously been shown, cocaine reinstated extinguished cocaine-taking behavior (Worley et al., 1994; Schenk and Partridge, 1999; Schenk et al., 1999). This effect was attenuated by pretreatment with KOPr agonists. High doses of SPR might have produced a nonselective effect on motor behavior as suggested by the decreased responding observed in a drug discrimination study (Holtzman et al., 1991). The attenuation of drug-seeking was also produced by a dose of SPR $(1.0 \mathrm{mg} / \mathrm{kg})$ that did not decrease responding in a drug discrimination task (Holtzman, 2000). It is therefore unlikely that the decrease in cocaineseeking represents a generalized inability to perform the lever press operant at the dose used in this study. Similarly, decreased drug-seeking was produced by doses of U69593 and U50488H that failed to produce a generalized decrease in motor activity (Schenk et al., 1999; unpublished findings).

In a previous study, $\mathrm{U} 50488 \mathrm{H}$ administered to mice $60 \mathrm{~min}$ prior to cocaine potentiated cocaine-induced conditioned place preference (CPP) but when administered 15 min prior to cocaine it suppressed the cocaineCPP (McLaughlin et al., 2006). In this study we measured cocaine-induced drug-seeking during a 60 min period following U50488H administration and also observed a small but non-significant increase in responding.

In drug discrimination tests, Sal A and its synthetic derivatives substituted completely for U69593 (Baker et al., 2009), suggesting an effect mediated by the KOPr. Because of the interaction between KOPr and dopaminergic mechanisms, a focus on the effects of Sal A on dopaminemediated behaviors has been of interest. Cocaine-seeking has been 

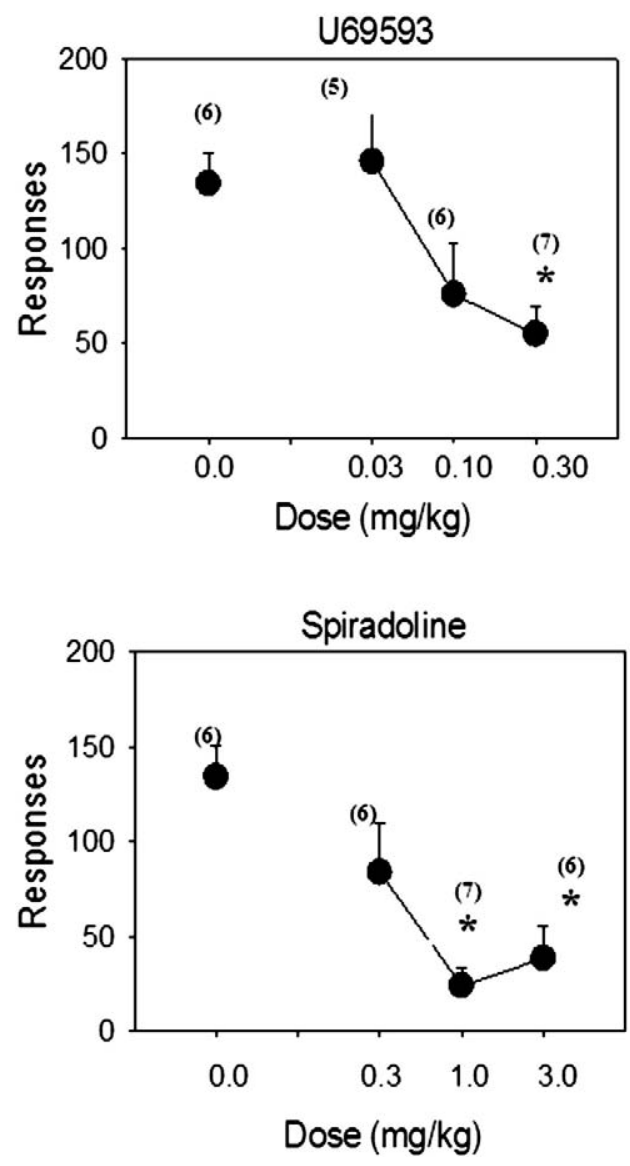

$\mathrm{U} 50488 \mathrm{H}$

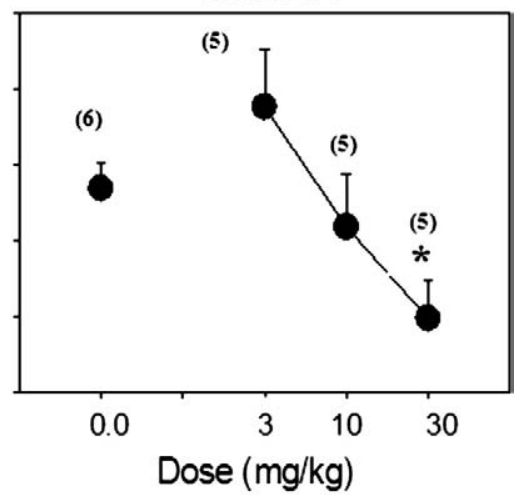

SalA

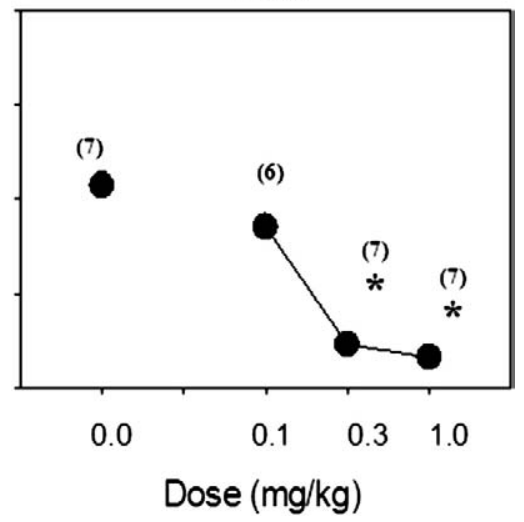

Fig. 2. Responses (+SEM) produced during phase 3 following injection of KOPr agonists and cocaine. Numbers in parentheses represent sample sizes for each group. ${ }^{*}<0.05$.

attributed to dopaminergic mechanisms and there are data to support the idea that Sal A modulates the effects of cocaine through interactions with D1 receptor mediated signaling in the dorsal striatum (Chartoff et al., 2008; Gehrke et al., 2008). Low doses of Sal A ( $40 \mu \mathrm{g} / \mathrm{kg}$ ) increased DA levels in nucleus accumbens (NAc) (Braida et al., 2008) whereas higher doses decreased NAc DA levels (Zhang et al., 2005). Thus, the ability of moderate to high doses of Sal A to decrease cocaine-produced drugseeking might be due to its effects on the dopaminergic system.

Alternatively, the decrease in drug-seeking might reflect a non specific effect. This possibility was tested by examining the effects of Sal A on responding maintained by a sucrose reinforcer. Conditions for

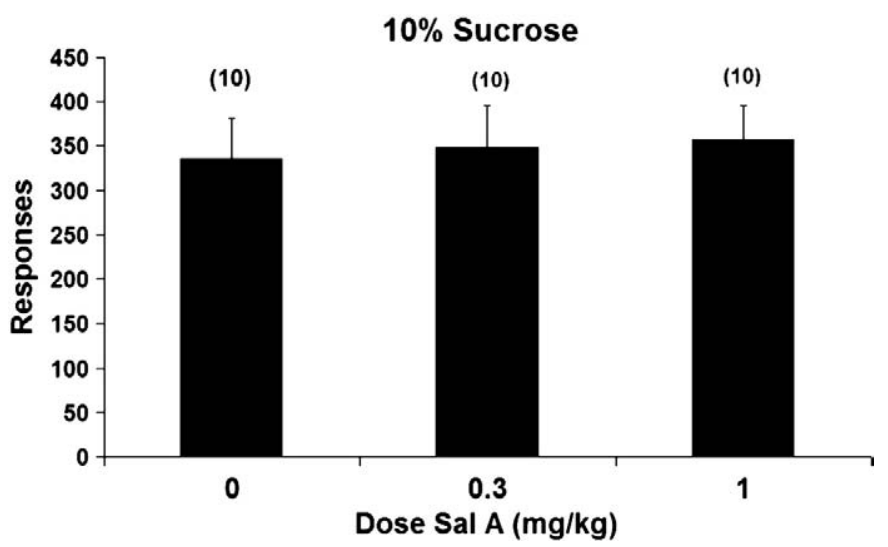

Fig. 3. Number of sucrose-reinforced responses $(+\mathrm{SEM})$ produced by rats for $60 \mathrm{~min}$ after treatment with Sal A ( 0.3 and $1.0 \mathrm{mg} / \mathrm{kg}$, I.P.). Numbers in parentheses represent sample sizes for each group. this test were comparable to conditions of the reinstatement tests. In this experiment, responding maintained by sucrose was high and comparable to responding maintained by cocaine. Doses of Sal A $(0.3$, $1.0 \mathrm{mg} / \mathrm{kg}$ I.P.) that decreased cocaine-seeking failed to alter sucrosereinforced responding.

Additionally, KOPr activation has shown to produce motor incoordination (Fantegrossi et al., 2005). A more recent report, however, failed to find any effect of Sal A on locomotor activity (Baker et al., 2009). The effect of Sal A on cocaine-produced hyperactivity was measured in the present study under conditions that were comparable to the conditions of the reinstatement tests. Sal A did not attenuate cocaineproduced hyperactivity. Therefore, the decrease in drug-seeking produced by Sal A cannot be attributed to either a generalized inability to perform the lever press operant or a disruption of motor activity.

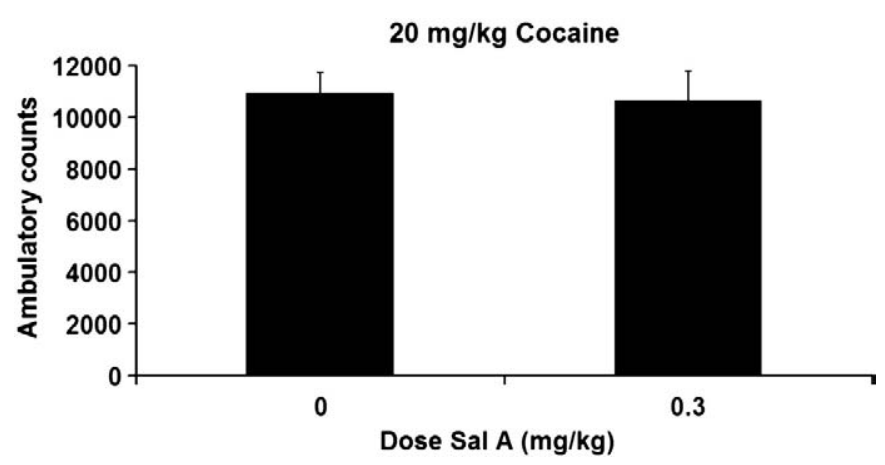

Fig. 4. Effect of Sal A ( 0 and $0.3 \mathrm{mg} / \mathrm{kg}$, I.P.) on cocaine-induced locomotion. Symbols represent total ambulatory counts ( + SEM) during the 60 min period following the cocaine injection $(n=6)$. 
Reinstatement of conditioned place preference (CPP) following extinction has also been used as a model of drug-seeking (Kreibich and Blendy, 2004) and a modulation of drug-seeking behaviors by traditional KOPr agonists has been demonstrated using this model. The effects appear, however, to be opposite to those produced when drug-seeking in a self-administration paradigm is measured. For example, foot-shock or forced swim stress was effective in reinstating both extinguished place preference (McLaughlin et al., 2003, 2006; Redila and Chavkin, 2008) and self-administration (Beardsley et al., 2005). The effect in the CPP paradigm was, however, blocked by pretreatment with the KOPr antagonist, norBNI (Redila and Chavkin, 2008; McLaughlin et al., 2003). The effect of KOPr antagonists might depend on the drug-seeking stimulus since the KOPr antagonist, JDTic, inhibited stress induced drug-seeking but had no effect on cocaine-produced reinstatement (Beardsley et al., 2005).

Further, KOPr knock out mice were insensitive to stress-produced reinstatement, as measured in the $\mathrm{CPP}$ paradigm, supporting the idea that reinstatement of $\mathrm{CPP}$ required KOPr activation. A further difference in the role of KOPr in reinstatement as measured in the CPP and self-administration paradigms is that $\mathrm{U} 50488 \mathrm{H}$ potentiated the cocaine-produced place preference whereas it decreased drugseeking following extinction of self-administration (present study). Additional studies will have to be conducted in order to tease apart the different aspects of the two paradigms.

In conclusion, Sal A, like traditional KOPr agonists attenuated cocaine-induced cocaine-seeking. Further studies on Sal A and its structural derivatives will help us understand the mechanism by which KOPr agonists attenuate drug-seeking behaviors and to determine whether Sal A produces the same magnitude of adverse effects that have limited the development of other KOPr agonists as anti-addiction pharmacotherapies.

\section{Acknowledgements}

This work was funded by Wellington Medical Research Foundation, The Neurological Foundation of New Zealand and grant DA018151 (T.E.P.) from the National Institute on Drug Abuse (NIDA). The authors are thankful for the technical assistance of Mr. Alex Howard, Mr. Lincoln Hely and Mr. Richard Moore.

\section{References}

Alleweireldt A, Weber SM, Kirschner KF, Bullock BL, Neisewander JL. Blockade or stimulation of D1 dopamine receptors attenuates cue reinstatement of extinguished cocaine-seeking behavior in rats. Psychopharmacology (Berl) 2002;159(3):284-93.

Baker L, Panos J, Killinger B, Peet M, Bell L, Haliw L, et al. Comparison of the discriminative stimulus effects of salvinorin A and its derivatives to U69593 and U50488 in rats. Psychopharmacology (Berl) 2009;203(3):203-11.

Beardsley PM, Howard JL, Shelton KL, Carroll FI. Differential effects of the novel kappa opioid receptor antagonist, JDTic, on reinstatement of cocaine-seeking induced by footshock stressors vs cocaine primes and its antidepressant-like effects in rats. Psychopharmacology (Berl) 2005;183(1):118-26.

Braida D, Limonta V, Capurro V, Fadda P, Rubino T, Mascia P, et al. Involvement of kappa-opioid and endocannabinoid system on salvinorin A-induced reward. Biol Psychiatry 2008;63(3):286-92.

Butelman ER, Harris T, Kreek MJ. The plant-derived hallucinogen, salvinorin A, produces kappa-opioid agonist-like discriminative effects in rhesus monkeys. Psychopharmacology (Berl) 2004;172(2):220-4.

Butelman ER, Prisinzano TE, Deng H, Rus S, Kreek MJ. Unconditioned behavioral effects of the powerful kappa-opioid hallucinogen salvinorin A in non-human primates: fast onset and entry into cerebrospinal fluid. J Pharmacol Exp Ther 2009;328(2):588-97.

Carlezon WJ, Beguin C, Dinieri JA, Baumann MH, Richards MR, Todtenkopf MS, et al. Depressive-like effects of the kappa-opioid receptor agonist salvinorin A on behavior and neurochemistry in rats. J Pharmacol Exp Ther 2006;316(1):440-7.

Chartoff EH, Potter D, Damez-Werno D, Cohen BM, Carlezon Jr WA. Exposure to the selective kappa-opioid receptor agonist salvinorin A modulates the behavioral and molecular effects of cocaine in rats. Neuropsychopharmacology 2008;33(11):2676-87.

Chavkin C, Sud S, Jin W, Stewart J, Zjawiony JK, Siebert DJ, et al. Salvinorin A, an active component of the hallucinogenic sage Salvia divinorum is a highly efficacious kappa-opioid receptor agonist: structural and functional considerations. J Pharmacol Exp Ther 2004;308(3):1197-203.

Devine DP, Leone P, Pocock D, Wise RA. Differential involvement of ventral tegmental mu, delta and kappa opioid receptors in modulation of basal mesolimbic dopamine release: in vivo microdialysis studies. J Pharmacol Exp Ther 1993;266 (3):1236-46.

Fantegrossi W, Kugle KM, Valdes III LJ, Koreeda M, Woods JH. Kappa-opioid receptormediated effects of the plant-derived hallucinogen, salvinorin $A$, on inverted screen performance in the mouse. Behav Pharmacol 2005;16(8):627-33.

Fischman MW, Schuster CR, Javaid J, Hatano Y, Davis J. Acute tolerance development to the cardiovascular and subjective effects of cocaine. J Pharmacol Exp Ther 1985;235(3):677-82.

Gehrke BJ, Chefer VI, Shippenberg TS. Effects of acute and repeated administration of salvinorin A on dopamine function in the rat dorsal striatum. Psychopharmacology (Berl) 2008;197(3):509-17.

Glick SD, Maisonneuve IM, Raucci J, Archer S. Kappa-opioid inhibition of morphine and cocaine self-administration in rats. Brain Res 1995;681(1-2):147-52.

Gray AM, Rawls SM, Shippenberg TS, McGinty JF. The kappa-opioid agonist, U69593, decreases acute amphetamine-evoked behaviors and calcium-dependent dialysate levels of dopamine and glutamate in the ventral striatum. J Neurochem 1999;73 (3):1066-74.

Hahn B, Stolerman IP, Shoaib M. Kappa-opioid receptor modulation of nicotine-induced behaviour. Neuropharmacology 2000;39(13):2848-55.

Heidbreder CA, Shippenberg TS. U69593 prevents cocaine sensitization by normalizing basal accumbens dopamine. NeuroReport 1994:5(14):1797-800.

Heidbreder CA, Goldberg SR, Shippenberg TS. The kappa-opioid receptor agonist U69593 attenuates cocaine-induced behavioral sensitization in the rat. Brain Res 1993;616(1-2):335-8.

Heidbreder CA, Babovic-Vuksanovic D, Shoaib M, Shippenberg TS. Development of behavioral sensitization to cocaine: influence of kappa-opioid receptor agonists. J Pharmacol Exp Ther 1995;275(1):150-63.

Heidbreder CA, Thompson AC, Shippenberg TS. Role of extracellular dopamine in the initiation and long-term expression of behavioral sensitization to cocaine. J Pharmacol Exp Ther 1996;278(2):490-502.

Holtzman SG. Further characterization of the discriminative stimulus effects of spiradoline. Pharmacol Biochem Behav 2000;66(3):517-22.

Holtzman SG, Cook L, Steinfels GF. Discriminative stimulus effects of spiradoline, a kappa-opioid agonist. Psychopharmacology (Berl) 1991;105(4):447-52.

Jaffe JH, Cascella NG, Kumor KM, Sherer MA. Cocaine-induced cocaine craving. Psychopharmacology (Berl) 1989;97(1):59-64.

John TF, French LG, Erlichman JS. The antinociceptive effect of salvinorin A in mice. Eur J Pharmacol 2006;545(2-3):129-33.

Khroyan TV, Barrett-Larimore RL, Rowlett JK, Spealman RD. Dopamine D1- and D2like receptor mechanisms in relapse to cocaine-seeking behavior: effects of selective antagonists and agonists. J Pharmacol Exp Ther 2000;294(2):680-7.

Khroyan TV, Platt DM, Rowlett JK, Spealman RD. Attenuation of relapse to cocaine seeking by dopamine D1 receptor agonists and antagonists in non-human primates. Psychopharmacology (Berl) 2003;168(1-2):124-31.

Kreibich AS, Blendy JA. cAMP response element-binding protein is required for stress but not cocaine-induced reinstatement. J Neurosci 2004;24(30):6686-92.

Maisonneuve IM, Archer S, Glick SD. U50488, a kappa-opioid receptor agonist, attenuates cocaine-induced increases in extracellular dopamine in the nucleus accumbens of rats. Neurosci Lett 1994;181(1-2):57-60.

McCurdy CR, Sufka KJ, Smith GH, Warnick JE, Nieto MJ. Antinociceptive profile of salvinorin $\mathrm{A}$, a structurally unique kappa opioid receptor agonist. Pharmacol Biochem Behav 2006;83(1):109-13.

McLaughlin JP, Marton-Popovici M, Chavkin C. Kappa opioid receptor antagonism and prodynorphin gene disruption block stress-induced behavioral responses. J Neurosci 2003;23(13):5674-83.

McLaughlin JP, Li S, Valdez J, Chavkin TA, Chavkin C. Social defeat stress-induced behavioral responses are mediated by the endogenous kappa opioid system. Neuropsychopharmacology 2006;31(6):1241-8.

Mello NK, Negus SS. Preclinical evaluation of pharmacotherapies for treatment of cocaine and opioid abuse using drug self-administration procedures. Neuropsychopharmacology 1996;14(6):375-424.

Mello NK, Negus SS. Effects of kappa-opioid agonists on cocaine- and food-maintained responding by rhesus monkeys. J Pharmacol Exp Ther 1998;286(2):812-24.

Negus SS, Mello NK, Portoghese PS, Lin CE. Effects of kappa-opioids on cocaine selfadministration by rhesus monkeys. J Pharmacol Exp Ther 1997;282(1):44-55.

Prisinzano TE. Psychopharmacology of the hallucinogenic sage Salvia divinorum. Life Sc 2005;78(5):527-31.

Prisinzano TE, Rothman RB. Salvinorin A analogues as probes in opioid pharmacology. Chem Rev 2008;108(5):1732-43.

Prisinzano TE, Tidgewell K, Harding WW. Kappa-opioids as potential treatments for stimulant dependance. AAPS J 2005;7(3):E592-9.

Redila VA, Chavkin C. Stress-induced reinstatement of cocaine seeking is mediated by the kappa opioid system. Psychopharmacology (Berl) 2008;200(1):59-70.

Roth BL, Baner K, Westkaemper R, Siebert D, Rice KC, Steinberg S, et al. Salvinorin A: a potent naturally occurring nonnitrogenous kappa-opioid selective agonist. Proc Natl Acad Sci U S A 2002;99(18):11934-9.

Schenk S, Partridge B. Cocaine-seeking produced by experimenter-administered drug injections: dose-effect relationships in rats. Psychopharmacology (Berl) 1999;147 (3):285-90

Schenk S, Partridge B. Effect of the kappa-opioid receptor agonist, U69593, on reinstatement of extinguished amphetamine self-administration behavior. Pharmacol Biochem Behav 2001;68(4):629-34. 
Schenk S, Partridge B, Shippenberg TS. U69593, a kappa-opioid agonist, decreases cocaine self-administration and decreases cocaine-produced drug-seeking. Psychopharmacology (Berl) 1999;144(4):339-46.

Schenk S, Partridge B, Shippenberg TS. Reinstatement of extinguished drug-taking behaviour in rats: effect of the kappa-opioid receptor agonist, U69593. Psychopharmacology (Berl) 2000;151(1):85-90.

Schmidt MD, Schmidt MS, Butelman ER, Harding WW, Tidgewell K, Murry DJ, et al. Pharmacokinetics of the plant-derived kappa-opioid hallucinogen salvinorin A in non-human primates. Synapse 2005;58(3):208-10.

Self DW, Barnhart WJ, Lehman DA, Nestler EJ. Opposite modulation of cocaine-seeking behavior by D1- and D2-like dopamine receptor agonists. Science 1996;271 (5255):1586-9.

Shippenberg TS, Spanagel R, Heidbreder CA. Modulation of mesolimbic dopamine release by endogeneous opioids; role in drug-induced sensitization and dependance. In: Louilot A, Durkin T, Spampinato U, Cardor M, editors. Monitoring molecules in neuroscienceProc. 6th international conference on in vivo methods; 1994. p. 123-5.

Shippenberg TS, LeFevour A, Heidbreder CA. Kappa-opioid receptor agonists prevent sensitization to the conditioned rewarding effects of cocaine. J Pharmacol Exp Ther 1996;276(2):545-54.

Shippenberg TS, Zapata A, Chefer VI. Dynorphin and the pathophysiology of drug addiction. Pharmacol Ther 2007;116(2):306-21.

Siebert DJ. Salvia divinorum and salvinorin A: new pharmacologic findings. J Ethnopharmacol 1994;43(1):53-6.

Thompson AC, Zapata A, Justice Jr JB, Vaughan RA, Sharpe LG, Shippenberg TS. Kappaopioid receptor activation modifies dopamine uptake in the nucleus accumbens and opposes the effects of cocaine. J Neurosci 2000;20(24):9333-40.
Tomasiewicz HC, Todtenkopf MS, Chartoff EH, Cohen BM, Carlezon Jr WA. The kappaopioid agonist U69,593 blocks cocaine-induced enhancement of brain stimulation reward. Biol Psychiatry 2008;64(11):982-8.

Valdes III LJ. Salvia divinorum and the unique diterpene hallucinogen, salvinorin (divinorin) A. J Psychoact Drugs 1994;26(3):277-83.

Valdés III LJ, Díaz J, Paul AG. Ethnopharmacology of ska María Pastora (Salvia divinorum, Epling AND Játiva-M.). J Ethnopharmacol 1983;7(3):287-312.

Willmore-Fordham $\mathrm{CB}$, Krall DM, McCurdy $\mathrm{CR}$, Kinder $\mathrm{DH}$. The hallucinogen derived from Salvia divinorum, salvinorin A, has kappa-opioid agonist discriminative stimulus effects in rats. Neuropharmacology 2007;53(4):481-6.

Wit $\mathrm{H}$, Stewart J. Reinstatement of cocaine-reinforced responding in the rat. Psychopharmacology (Berl) 1981;75(2):134-43.

Worley CM, Valadez A, Schenk S. Reinstatement of extinguished cocaine-taking behavior by cocaine and caffeine. Pharmacol Biochem Behav 1994;48(1):217-21.

$\mathrm{Xi}$ Z-X, Fuller SA, Stein EA. Dopamine release in the nucleus accumbens during heroin self-administration is modulated by kappa opioid receptors: an in vivo fast-cyclic voltammetry study. J Pharmacol Exp Ther 1998;284(1):151-61.

Yan F, Roth BL. Salvinorin A: a novel and highly selective kappa-opioid receptor agonist. Life Sci 2004;75(22):2615-9.

Zhang Y, Butelman ER, Schlussman SD, Ho A, Kreek MJ. Effects of the plant-derived hallucinogen salvinorin A on basal dopamine levels in the caudate putamen and in a conditioned place aversion assay in mice: agonist actions at kappa-opioid receptors. Psychopharmacology (Berl) 2005;179(3):551-8. 